Supporting Information

\title{
Increasing Fluorous Partition Coefficients by Solvent Tuning
}

\author{
Marvin S. $\mathrm{Yu}^{1}$, Dennis P. Curran ${ }^{2}$, and Tadamichi Nagashima ${ }^{1}$

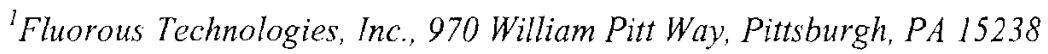 \\ ${ }^{2}$ Department of Chemistry, University of Pittsburgh, Pittsburgh, PA 15260
}

General: All solvents were reagent grade and used without further purification. THF and DMF were purchased from Aldrich. $\mathrm{CH}_{3} \mathrm{CN}, \mathrm{MeOH}$, and diethyl ether were purchased from Fisher Scientific. FC-72 and HFE-7100 were purchased from 3M.

Gas chromatograms were obtained using a HP 5890 Series II GC equipped with a HP 7673 Autosampler, HP 6890 Integrator, and a Agilent HP-1 column (part\# 19091Z, 30m x $0.32 \mathrm{~mm}, 25$ micron).

HPLC chromatograms were obtained using an Agilent 1100 Series HPLC with the Agilent Chemstation software. The general method was:

Column: Phenomenex Luna C18, $50 \times 2.1 \mathrm{~mm}, 3 \mathrm{um}$

Solvent A: $0.05 \%$ TFA in water

Solvent B: $0.05 \%$ TFA in $\mathrm{MeCN}$

Detection: $254 \mathrm{~nm}$

Injection volume: $2 \mu \mathrm{L}$

Temperature: $40^{\circ} \mathrm{C}$

Flow rate: $1 \mathrm{ml} / \mathrm{min}$

Gradient: $0-95 \% \mathrm{~B}$ over $8 \mathrm{~min}, 2$ min re-equilibration

NMR spectra were obtained on a $270 \mathrm{MHz}$ Bruker. Chemical shifts are reported in parts per million $(\mathrm{ppm}, \delta)$ and are referenced to the residual solvent. Coupling constants are reported in Hertz $(\mathrm{Hz})$. Spectral splitting patterns are designated as s: singlet, d: doublet, t: triplet, q: quartet, m: complex multiplet.

Synthesis of previously unreported compounds is briefly described within. Purities were assessed by ${ }^{1} \mathrm{H}$ NMR and/or ${ }^{13} \mathrm{C}$ NMR. MS data collected using APCI as the ion source. HRMS data collected using EI.

Calculated Log P (octanol-water) was determined using ChemOffice Ultra 2005. 
General Procedure for Determination of Partitioning Coefficients: $50 \mathrm{mg}$ of compound was dissolved in $1 \mathrm{~mL}$ each of the appropriate organic solvent and the fluorous solvent. The biphasic mixture was stirred for 15 minutes and the phases allowed to separate. A $50 \mu \mathrm{L}$ aliquot of each phase was dissolved in $1 \mathrm{~mL}$ of THF. The resulting analytical solutions were analyzed by either GC or LC $(254 \mathrm{~nm})$. Partition coefficients were determined by comparing the absolute area counts for each phase.

Example: Table 1, Entry 4. Fluorous phase GC area count $=16,418$. Organic phase GC area count $=131,643 . p=16,418 / 131,643=0.12$.

Triphenylphosphine was purchased from Aldrich.

Phenylbis [4-(3,3,4,4,5,5,6,6,7,7,8,8,8-tridecafluorooctyl)-phenyl]phosphane Prepared according to Zhang, Q.; Luo, Z.; Curran, D.P. J.Org. Chem. 2000, 65, 8866.

(1).

\section{$N$-[3-(Perfluorohexyl)propyl]- $N$-[3-(perfluorooctyl)propyl]-4-chloro-3-}

nitrobenzenesulfonamide (2): To a mixture of 3-(perfluorooctyl)propylamine $(4.78 \mathrm{~g}$, $10.0 \mathrm{mmol})$ and $N, N$-diisopropylethylamine $(2.0 \mathrm{~mL}, 11 \mathrm{mmol})$ in THF $(10 \mathrm{~mL})$ was added 4-chloro-3-nitrobenzenesulfonyl chloride $(2.57 \mathrm{~g}, 10.0 \mathrm{mmol})$ at $23{ }^{\circ} \mathrm{C}$. After 30 min, $\mathrm{Et}_{2} \mathrm{O}(50 \mathrm{~mL})$ and THF $(20 \mathrm{~mL})$ were added. It was washed with $1 \mathrm{~N} \mathrm{HCl}(2 \mathrm{x})$, $\mathrm{H}_{2} \mathrm{O}(1 \mathrm{x})$, and brine $(1 \mathrm{x})$. It was dried over $\mathrm{MgSO}_{4}$. After removal of the solvent, light yellow solid was obtained that was used for the next step. Yield $=6.28 \mathrm{~g}(6.0 \mathrm{mmol}$, $90 \%$ yield).

A mixture of $N$-[3-(Perfluorooctyl)propyl]-4-chloro-3-nitrobenzenesulfonamide (1.39 g, $2.00 \mathrm{mmol}$ ), $\mathrm{Ph}_{3} \mathrm{P}$ (0.69 g, $2.6 \mathrm{mmol}$ ), 3-(perfluorohexyl)propanol (0.91 g, $2.4 \mathrm{mmol}$ ) in THF ( $5 \mathrm{~mL}$ ) was cooled on an icc-water bath, and then diisopropyl azodicarboxylate $(0.50 \mathrm{~mL}, 2.5 \mathrm{mmol})$ was added over the period of $2 \mathrm{~min}$. After the addition, the cooling bath was removed. After $2 \mathrm{~d}, \mathrm{Et}_{2} \mathrm{O}(10 \mathrm{~mL})$ was added. It was washed with $3 \%$ aqueous $\mathrm{H}_{2} \mathrm{O}_{2}(1 \mathrm{x}), 1 \mathrm{~N} \mathrm{NaOH}(1 \mathrm{x}), \mathrm{H}_{2} \mathrm{O}(1 \mathrm{x})$, and brine $(1 \mathrm{x})$. It was dried over $\mathrm{MgSO}_{4}$. Most of triphenylphosphine oxide was removed by passing though a plug of $\mathrm{SiO}_{2}(12 \mathrm{~g}$, eluent $=$ hexanes - EtOAc, 4:1). The filtrate was concentrated. The product was purified by recrystallization from $\mathrm{MeOH}$ to give white solid: Yield $1.18 \mathrm{~g}(56 \%)$. ${ }^{1} \mathrm{H}$ NMR $(270$ $\left.\mathrm{MHz} \mathrm{CDCl}_{3}\right) \delta=8.29(1 \mathrm{H}, \mathrm{d}, J=2.1 \mathrm{~Hz}), 7.93(1 \mathrm{H}, \mathrm{dd}, J=2.2,8.5 \mathrm{~Hz}), 7.77(1 \mathrm{H}, \mathrm{d}, J$ $=8.4 \mathrm{~Hz}), 3.26(4 \mathrm{H}, \mathrm{t}, J=7.1 \mathrm{~Hz}), 2.30-2.07(4 \mathrm{H}, \mathrm{m}), 2.02-1.87(4 \mathrm{H}, \mathrm{m}) .{ }^{13} \mathrm{C} \mathrm{NMR}$ $\left(67.5 \mathrm{MHz} \mathrm{CDCl}_{3}\right) \delta=148.1,139.1,133.3,132.1,131.0,124.4,121-106(\mathrm{~m}), 48.8$, $28.1(\mathrm{t}, J=22.3 \mathrm{~Hz}), 20.4$. HRMS calculated for $\mathrm{C}_{26} \mathrm{H}_{16} \mathrm{ClF}_{30} \mathrm{~N}_{2} \mathrm{O}_{4} \mathrm{~S}$; Calculated mass $=$ 1057.0040. Found $=1057.0150$.

N-Octyl-4-chloro-3-nitrobenzenesulfonamide (3): To a mixture of octylamine (3.8 $\mathrm{mL}, 23 \mathrm{mmol})$ and $N, N$-diisopropylethylamine $(4.6 \mathrm{~mL}, 26 \mathrm{mmol})$ in dichloromethane $(60 \mathrm{~mL})$ was added 4-chloro-3-nitrobenzenesulfonyl chloride $(5.24 \mathrm{~g}, 20.5 \mathrm{mmol})$ in small portions over the period of $9 \mathrm{~min}$ at $23{ }^{\circ} \mathrm{C}$. After $1 \mathrm{~h}, \mathrm{H}_{2} \mathrm{O}(100 \mathrm{~mL})$ was added. The mixture was extracted with $\mathrm{Et}_{2} \mathrm{O}(2 \times 60 \mathrm{~mL})$. The ether layers were combined, and were washed with $0.2 \mathrm{~N} \mathrm{HCl}(2 \mathrm{x})$ and brine $(1 \mathrm{x})$. It was dried over $\mathrm{MgSO}_{4}$. After the removal of the solvent, yellow powder was obtained: Yield $=6.48 \mathrm{~g}(18.5 \mathrm{mmol}, 90 \%)$. 
${ }^{1} \mathrm{H}$ NMR $\left(270 \mathrm{MHz}, \mathrm{CDCl}_{3}\right) \delta=8.36(1 \mathrm{H}, \mathrm{d}, J=2.1 \mathrm{~Hz}), 7.99(1 \mathrm{H}, \mathrm{dd}, J=2.4,8.5 \mathrm{~Hz})$, $7.73(1 \mathrm{H}, \mathrm{d}, J=8.3 \mathrm{~Hz}), 4.67(1 \mathrm{H}, \mathrm{t}, J=6.0 \mathrm{~Hz}), 3.02(2 \mathrm{H}, \mathrm{q}, J=7.1 \mathrm{~Hz}), 1.53-1.44(2$ $\mathrm{H}, \mathrm{m}), 1.23\left(10 \mathrm{H}\right.$, br s) $0.87(3 \mathrm{H}, \mathrm{t}, J=6.5 \mathrm{~Hz}) .{ }^{13} \mathrm{C} \mathrm{NMR}\left(67.5 \mathrm{MHz}, \mathrm{CDCl}_{3}\right) \delta=$ $147.8,140.4,133.0,131.5,131.1,124.4,43.4,31.7,29.5,29.1,29.0,26.5,22.6,14.1$.

Sulfathiazole(4): Purchased from Aldrich

2-bromo- $\boldsymbol{N}$-[4-[(2-thiazolylamino)sulfonyl]-phenylacetamide (5). Prepared as described by Bierer, D.E.; Moinet, G.G.; Botton, G.; Dubenko, L.; Patereau, G.; Doare, L.; Kergoat, M.; Meangeau, D.; Lu, Q. Piperizine Derivatives Useful as Hypoglycemic Agents. PCT Int. App1. 1999.

2-chloro-5-(N-methylsulfamoyl)-nitrobenzene(6): Purchased from Clariant Corp.

$N$-(4-fluorophenyl)- $N^{\prime}$-[(4-methoxyphenyl)methyl]-urea

(7).

4fluorophenylisocyanate (Aldrich, $200 \mu \mathrm{L}, 1.78 \mathrm{mmol}$ ) was dissolved in $\mathrm{CH}_{2} \mathrm{Cl}_{2}(3 \mathrm{~mL}$ ) and cooled to $0^{\circ} \mathrm{C}$. 4-methoxybenzylamine $(254 \mu \mathrm{L}, 1.96 \mathrm{mmol}, 1.1$ equiv.) was added and the mixture allowed to stir for $1 \mathrm{~h}$. The mixture was diluted with $\mathrm{Et}_{2} \mathrm{O}(10 \mathrm{~mL})$ and washed with $1.0 \mathrm{~N} \mathrm{HCl}(2 \times 5 \mathrm{~mL})$, aq. $\mathrm{NaHCO}_{3}(1 \times 5 \mathrm{~mL})$, and dried over $\mathrm{MgSO}_{4}$. Rotary evaporation and drying in vacuo gave a white solid. Yield $=412 \mathrm{mg}(91 \%)$. ' $\mathrm{H}$ NMR (270 MHz, DMSO-d $\left.{ }_{6}\right) \delta 8.60(\mathrm{~s}, 1 \mathrm{H}), 7.42$ (apparent $\left.\mathrm{q}, \mathrm{J}=4.9,9.0 \mathrm{~Hz}, 2 \mathrm{H}\right), 7.24$ $(\mathrm{d}, J=8.0 \mathrm{~Hz}, 2 \mathrm{H}), 7.08(\mathrm{t}, J=7.1 \mathrm{~Hz}, 2 \mathrm{H}), 6.90(\mathrm{~d}, J=7.6 \mathrm{~Hz}, 2 \mathrm{H}), 6.55(\mathrm{t}, J=5.7 \mathrm{~Hz}$, $1 \mathrm{H}), 4.22(\mathrm{~d}, J=5.7 \mathrm{~Hz}, 2 \mathrm{H}), 3.73(\mathrm{~s}, 3 \mathrm{H}) .{ }^{13} \mathrm{C}$ NMR $\left(67.5 \mathrm{~Hz}, \mathrm{DMSO}-\mathrm{d}_{6}\right) \delta 158.67$, $156.7\left(\mathrm{~d}, J_{C F}=240 \mathrm{~Hz}\right), 136.9,132.3,128.6,119.3,115.1\left(\mathrm{~d}, J_{C C F}=20 \mathrm{~Hz}\right), 113.7$, 55.06, 42.5. HRMS calculated for $\mathrm{C}_{15} \mathrm{H}_{15} \mathrm{FN}_{2} \mathrm{O}_{2} ;$ Calculated mass $=274.111756$. Found $=274.112114$.

$N$-(4-fluorophenyl)-4-methoxy-benzeneacetamide (8). 4-fluorobenzoyl chloride (200 $\mu \mathrm{L}, 1.66 \mathrm{mmol})$ was dissolved in $\mathrm{CH}_{2} \mathrm{Cl}_{2}(3 \mathrm{~mL})$ and cooled to $0^{\circ} \mathrm{C}$. A solution of 4methoxybenzyl amine (283 $\mu \mathrm{L}, 1.83 \mathrm{mmol}, 1.1$ equiv.), triethylamine (277 $\mu \mathrm{L}, 1.99$ mmol, 1.2 equiv.), and DMAP (2 mg) in $\mathrm{CH}_{2} \mathrm{Cl}_{2}(2 \mathrm{~mL})$ was added to the cooled solution of 4-fluorobenzoyl chloride. After 1 h reaction was deemed complete by GC analysis. The mixture was diluted with diethyl ether $(10 \mathrm{~mL})$ and washed with water $(1 \mathrm{x} 10 \mathrm{~mL})$, $1.0 \mathrm{~N} \mathrm{HCl}(2 \times 10 \mathrm{~mL})$, and saturated aqueous $\mathrm{NaHCO}_{3}(1 \times 10 \mathrm{~mL})$. The organic layer was dried over $\mathrm{MgSO}_{4}$, filtered, and the solvent ratoary evaporated. Drying in vacuo provided a white solid. Yield $=402 \mathrm{mg}(93 \%)$. ${ }^{1} \mathrm{H} \mathrm{NMR}\left(270 \mathrm{MHz}, \mathrm{CDCl}_{3}\right)$ o 7.80 (apparent q, $\mathrm{J}=5.4,8.5 \mathrm{~Hz}, 2 \mathrm{H}), 7.22(\mathrm{~d}, J=8.5 \mathrm{~Hz}, 2 \mathrm{H}), 7.05(\mathrm{t}, J=8.5 \mathrm{~Hz}, 2 \mathrm{H}), 6.83$ $(\mathrm{d}, J=8.5 \mathrm{~Hz}, 2 \mathrm{H}), 4.50(\mathrm{~d}, J=5.4 \mathrm{~Hz}, 2 \mathrm{H}), 3.77(\mathrm{~s}, 3 \mathrm{H}) .{ }^{13} \mathrm{C} \mathrm{NMR}\left(67.5 \mathrm{~Hz}, \mathrm{CDCl}_{3}\right) \delta$ $166.34,160.12\left(\mathrm{~d}, J_{C F}=240 \mathrm{~Hz}\right), 130.39,130.13,129.36,129.23,129.02,115.48$, $115.15,113.87,55.12,43.47$. HRMS calculated for $\mathrm{C}_{15} \mathrm{H}_{14} \mathrm{FNO}_{2}$; Calculated mass $=$ 259.100857 . Found $=259.100550$.

1-(4-methoxy-benzoyl)-4-phenyl-piperazine (9). 4-methoxybenzoyl chloride $(200 \mu \mathrm{L}$, $1.85 \mathrm{mmol})$ was dissolved in $\mathrm{CH}_{2} \mathrm{Cl}_{2}(3 \mathrm{~mL})$ and cooled to $0^{\circ} \mathrm{C}$. A solution of N-phenylpiperizine $(281 \mu \mathrm{L}, 1.85 \mathrm{mmol}, 1.0$ equiv.), triethylamine $(310 \mu \mathrm{L}, 2.22 \mathrm{mmol}, 1.2$ equiv.), and DMAP (2 mg) in $\mathrm{CH}_{2} \mathrm{Cl}_{2}(2 \mathrm{~mL})$ was added to the cooled solution of 4methoxybenzoyl chloride. After $1 \mathrm{~h}$ reaction was deemed complete by $\mathrm{GC}$ analysis. The 
mixture was diluted with diethyl ether $(10 \mathrm{~mL})$ and washed with water $(1 \times 10 \mathrm{~mL}), 1.0$ $\mathrm{N} \mathrm{HCl}(2 \times 10 \mathrm{~mL})$, and saturated aqueous $\mathrm{NaHCO}_{3}(1 \times 10 \mathrm{~mL})$. The organic layer was dried over $\mathrm{MgSO}_{4}$, filtered, and the solvent ratoary evaporated. Drying in vacuo provided a white solid. Yield $=527 \mathrm{mg}(96 \%)$. ${ }^{\mathrm{l}} \mathrm{H} \mathrm{NMR}\left(270 \mathrm{MHz}, \mathrm{CDCl}_{3}\right) \delta 7.46(\mathrm{~d}, \mathrm{~J}$ $=8.5 \mathrm{~Hz}, 2 \mathrm{H}), 7.32(\mathrm{t}, J=7.8 \mathrm{~Hz}, 2 \mathrm{H}), 6.96(\mathrm{~d}, J=8.3 \mathrm{~Hz}, 5 \mathrm{H}), 3.84(\mathrm{~s}, 3 \mathrm{H}), 3.74($ br s, $4 \mathrm{H}), 3.21$ (br s, $4 \mathrm{H}) .{ }^{13} \mathrm{C} \operatorname{NMR}\left(67.5 \mathrm{~Hz}, \mathrm{CDCl}_{3}\right) \delta 170.23,160.27,150.87,129.13$, $127.45,116.54,113.63,55.24,49.55$. HRMS calculated for $\mathrm{C}_{18} \mathrm{H}_{20} \mathrm{~N}_{2} \mathrm{O}_{2}$; Calculated mass $=296.152478$. Found $=296.153494$.

$N$-(4-fluorophenyl)-4-phenyl-1-piperazinecarboxamide (10). 4-fluorobenzoyl chloride $(200 \mu \mathrm{L}, 1.66 \mathrm{mmol})$ was dissolved in $\mathrm{CH}_{2} \mathrm{Cl}_{2}(3 \mathrm{~mL})$ and cooled to $0^{\circ} \mathrm{C}$. A solution of $\mathrm{N}$-phenyl-piperizine $(247 \mu \mathrm{L}, 1.85 \mathrm{mmol}, 1.0$ equiv.), triethylamine $(277 \mu \mathrm{L}, 1.83 \mathrm{mmol}$, 1.2 equiv.), and DMAP ( $2 \mathrm{mg})$ in $\mathrm{CH}_{2} \mathrm{Cl}_{2}(2 \mathrm{~mL})$ was added to the cooled solution of 4 fluorobenzoyl chloride. After $1 \mathrm{~h}$ reaction was deemed complete by $\mathrm{GC}$ analysis. The mixture was diluted with diethyl ether $(10 \mathrm{~mL})$ and washed with water $(1 \times 10 \mathrm{~mL}), 1.0$ $\mathrm{N} \mathrm{HCl}(2 \times 10 \mathrm{~mL})$, and saturated aqueous $\mathrm{NaHCO}_{3}(1 \times 10 \mathrm{~mL})$. The organic layer was dried over $\mathrm{MgSO}_{4}$, filtered, and the solvent ratoary evaporated. Drying in vacuo provided a white solid. Yield $=423 \mathrm{mg}(90 \%)$. ${ }^{1} \mathrm{H}$ NMR $\left(270 \mathrm{MHz}, \mathrm{CDCl}_{3}\right) \hat{o} 7.50(\mathrm{~m}$, 2H), $7.32(\mathrm{~m}, 2 \mathrm{H}), 7.15(\mathrm{~m}, 2 \mathrm{H}), 6.96(\mathrm{~m}, 3 \mathrm{H}), 3.96$ (br s, 2H), 3.74 (br s, 2H), 3.19 (br s, 4H). ${ }^{13} \mathrm{C} \mathrm{NMR}\left(67.5 \mathrm{~Hz}, \mathrm{CDCl}_{3}\right) \delta 169.46,163.1\left(\mathrm{~d}, J_{\mathrm{CF}}=240 \mathrm{~Hz}\right), 150.82,132.41$, $129.48,129.42,120.68,116.72,115.79,115.47,49.17$. HRMS calculated for $\mathrm{C}_{17} \mathrm{H}_{17} \mathrm{FN}_{2} \mathrm{O}$; Calculated mass $=284.132492$. Found $=284.132562$.

3-phenyl-2-propynyl-p-methoxy-benzoate (11). 4-methoxy-benzoyl chloride (200 $\mu \mathrm{L}$, $1.85 \mathrm{mmol})$ was dissolved in $\mathrm{CH}_{2} \mathrm{Cl}_{2}(3 \mathrm{~mL})$ and cooled to $0^{\circ} \mathrm{C}$. A solution of propargyl alcohol $(225 \mu \mathrm{L}, 1.85 \mathrm{mmol}, 1.0$ equiv.), triethylanine ( $310 \mu \mathrm{L}, 12.22 \mathrm{mmol}, 1.2$ equiv.), and DMAP $(2 \mathrm{mg})$ in $\mathrm{CH}_{2} \mathrm{Cl}_{2}(2 \mathrm{~mL})$ was added to the cooled solution of 4-methoxybenzoyl chloride. After $1 \mathrm{~h}$ reaction was deemed complete by $\mathrm{GC}$ analysis. The mixture was diluted with diethyl ether $(10 \mathrm{~mL})$ and washed with water $(1 \times 10 \mathrm{~mL}), 1.0 \mathrm{~N} \mathrm{HCl}(2$ $\times 10 \mathrm{~mL})$, and saturated aqueous $\mathrm{NaHCO}_{3}(1 \times 10 \mathrm{~mL})$. The organic layer was dried over $\mathrm{MgSO}_{4}$, filtered, and the solvent ratoary evaporated. Drying in vacuo provided a brown oil. Yield $=422 \mathrm{mg}(86 \%)$. ${ }^{\mathrm{l}} \mathrm{H}$ NMR $\left(270 \mathrm{MHz}, \mathrm{CDCl}_{3}\right) \hat{\delta} 8.10(\mathrm{~m}, 2 \mathrm{H}), 7.53(\mathrm{~m}, 2 \mathrm{H})$, $7.35(\mathrm{~m}, 3 \mathrm{H}), 6.94(\mathrm{~m} 2 \mathrm{H}), 5.18(\mathrm{~s}, 2 \mathrm{H}), 3.88(\mathrm{~s}, 3 \mathrm{H}) .{ }^{13} \mathrm{C} \mathrm{NMR}\left(67.5 \mathrm{~Hz}, \mathrm{CDCl}_{3}\right) \delta$ $165.59,163.44,131.78,128.61,128.17,122.07,121.79,113.52,86.26,83.16,55.32$, 52.94. 

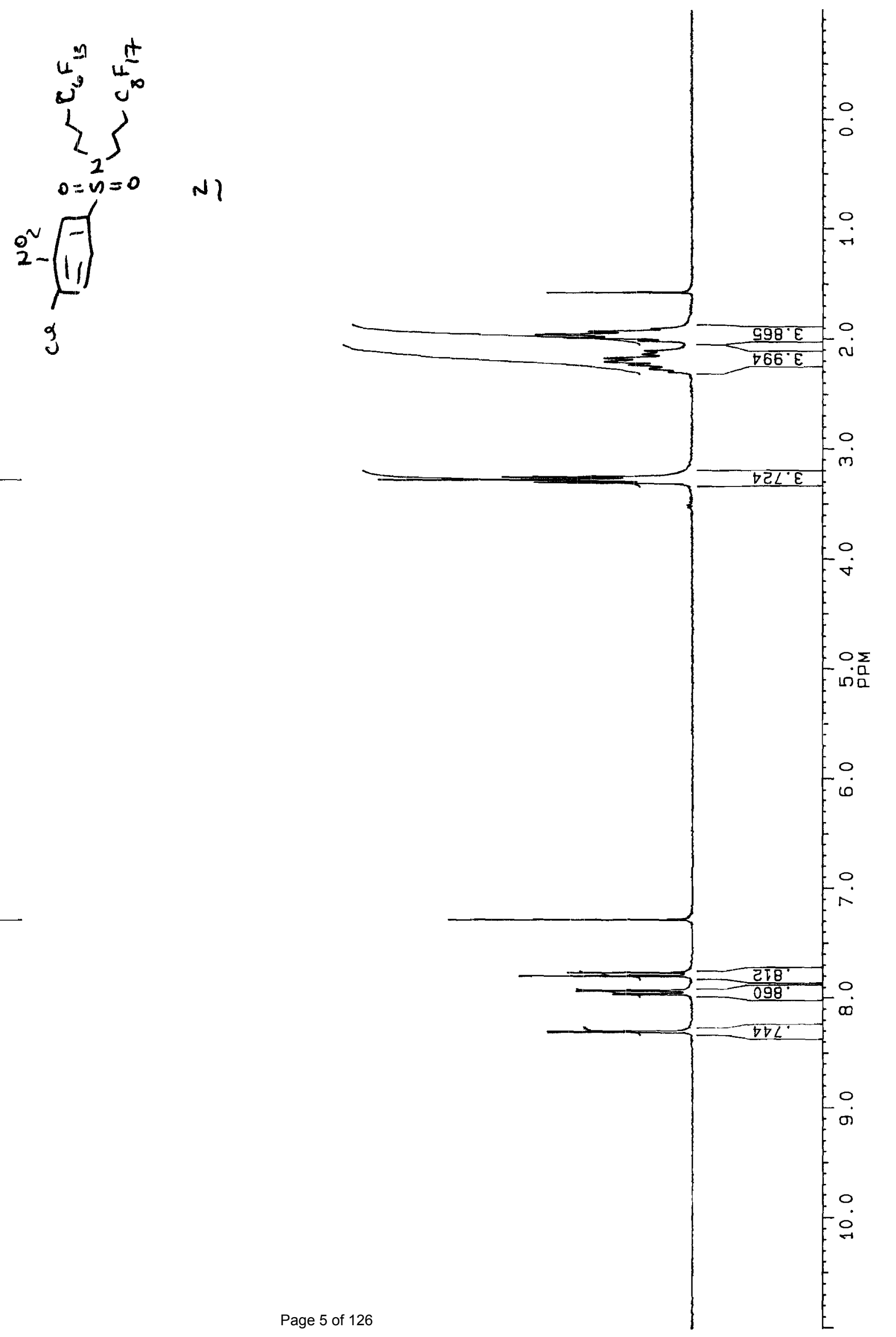

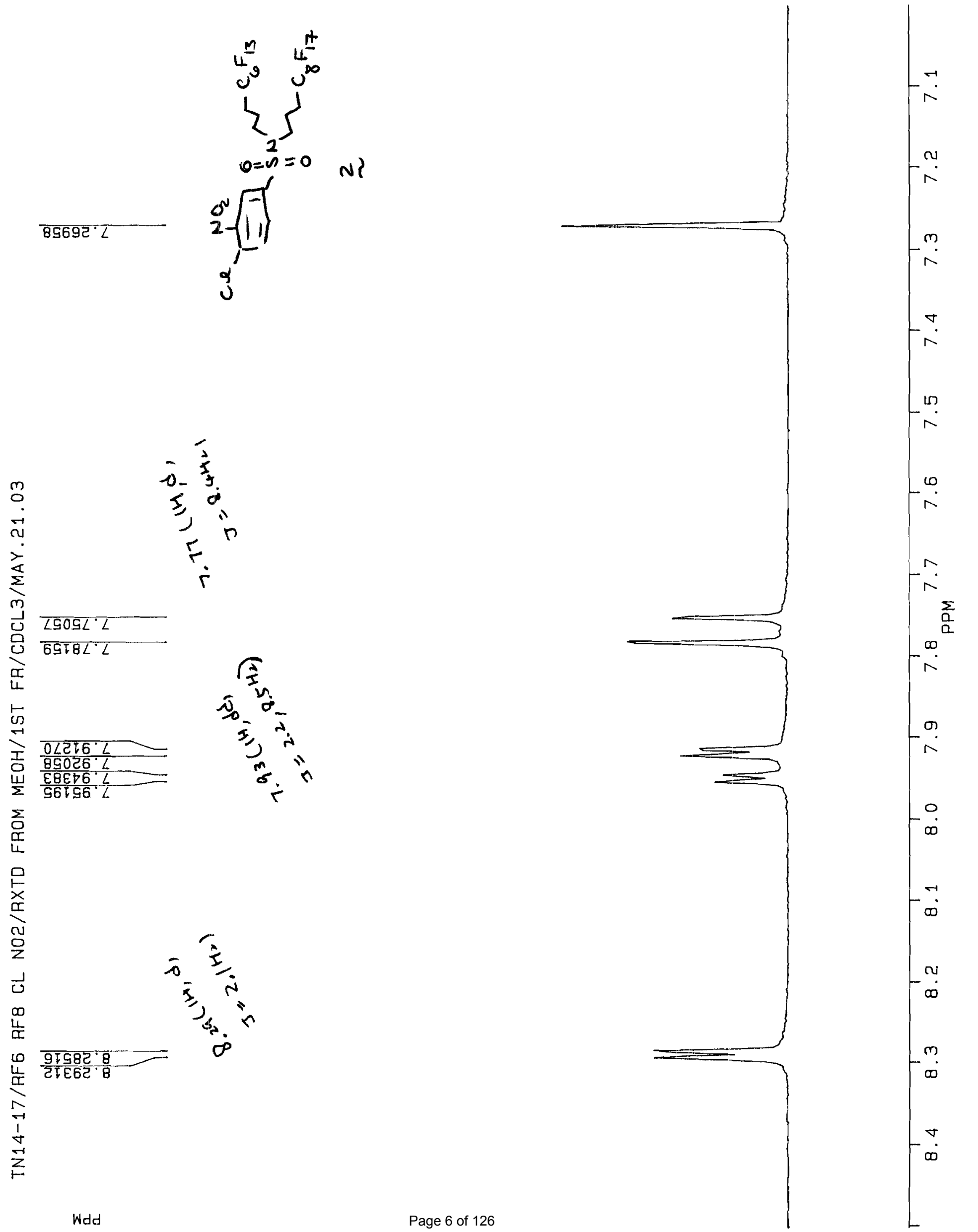

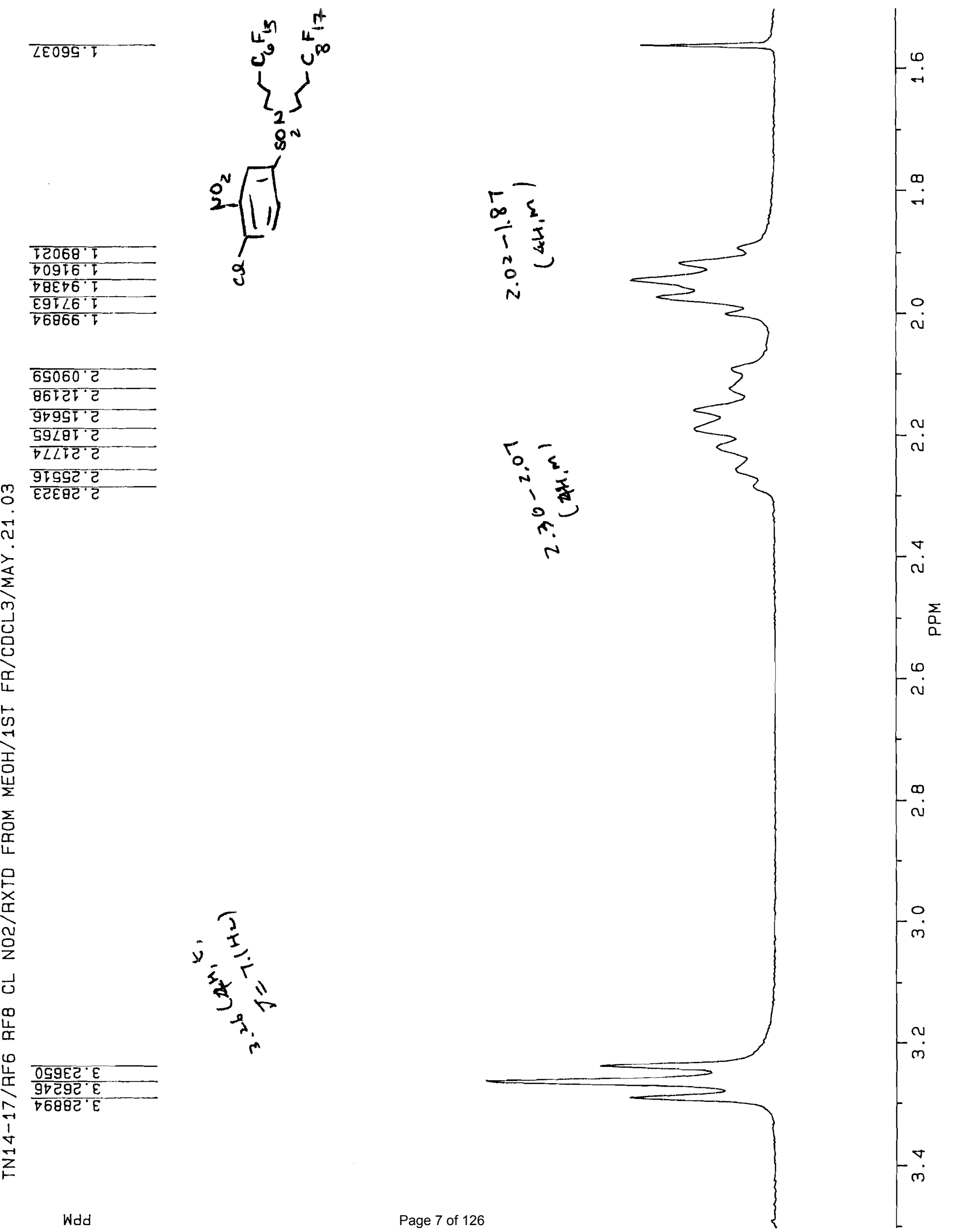


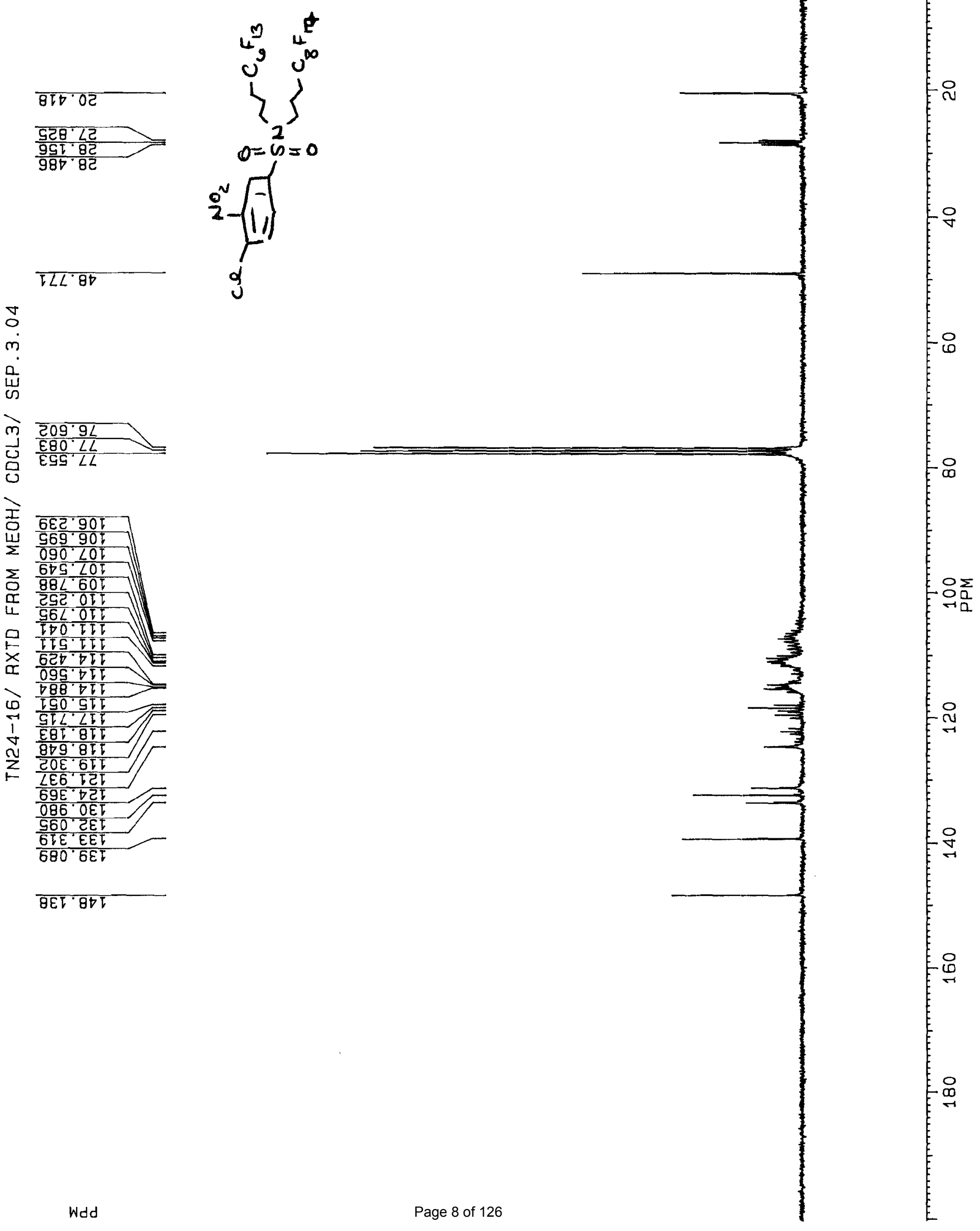



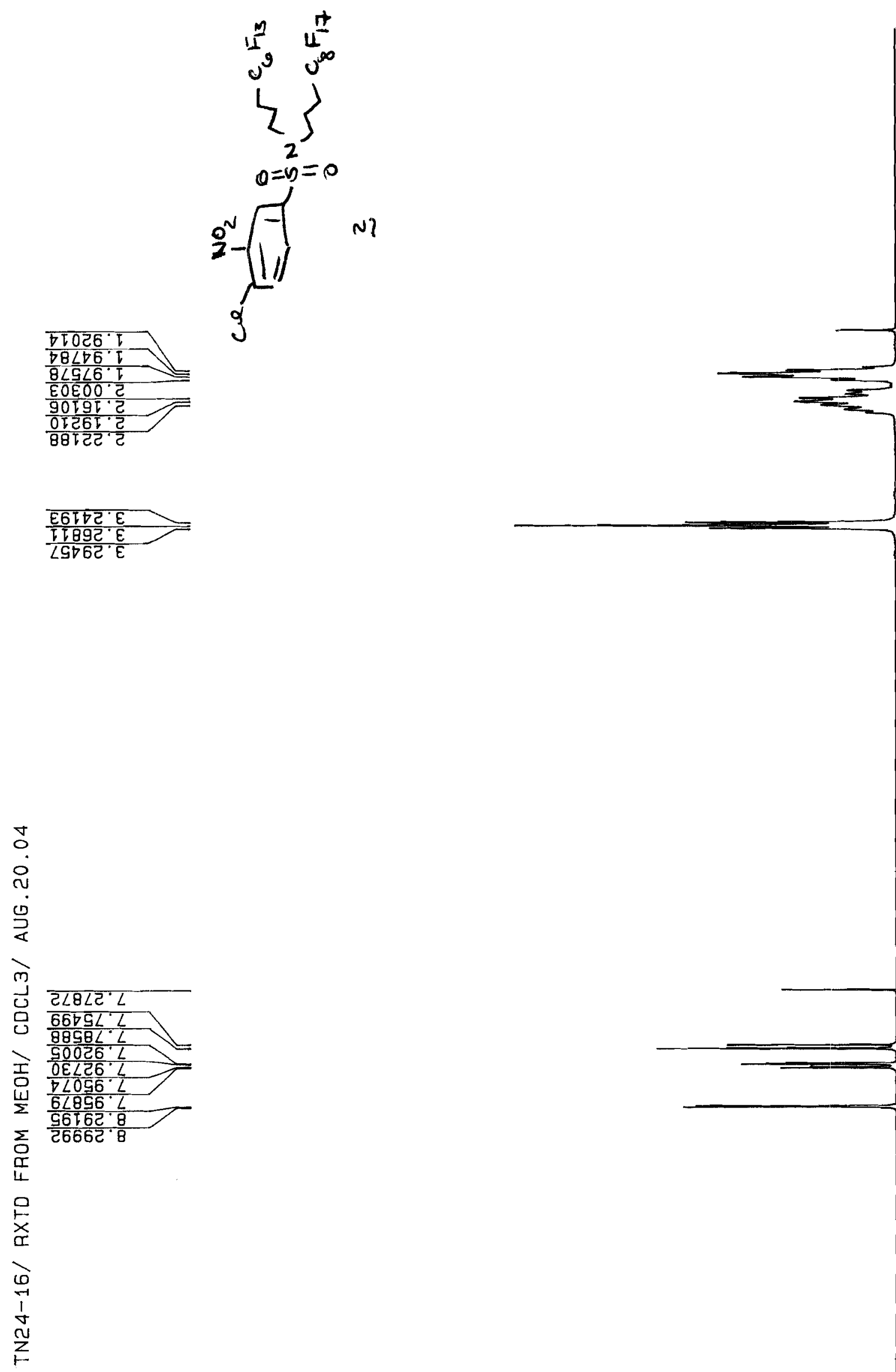


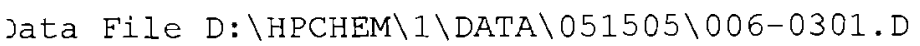

$\mathrm{Rf} 6+\mathrm{Rf} 8 / \mathrm{Cl}-\mathrm{NO} 2 /$ in $\mathrm{MeOH}$
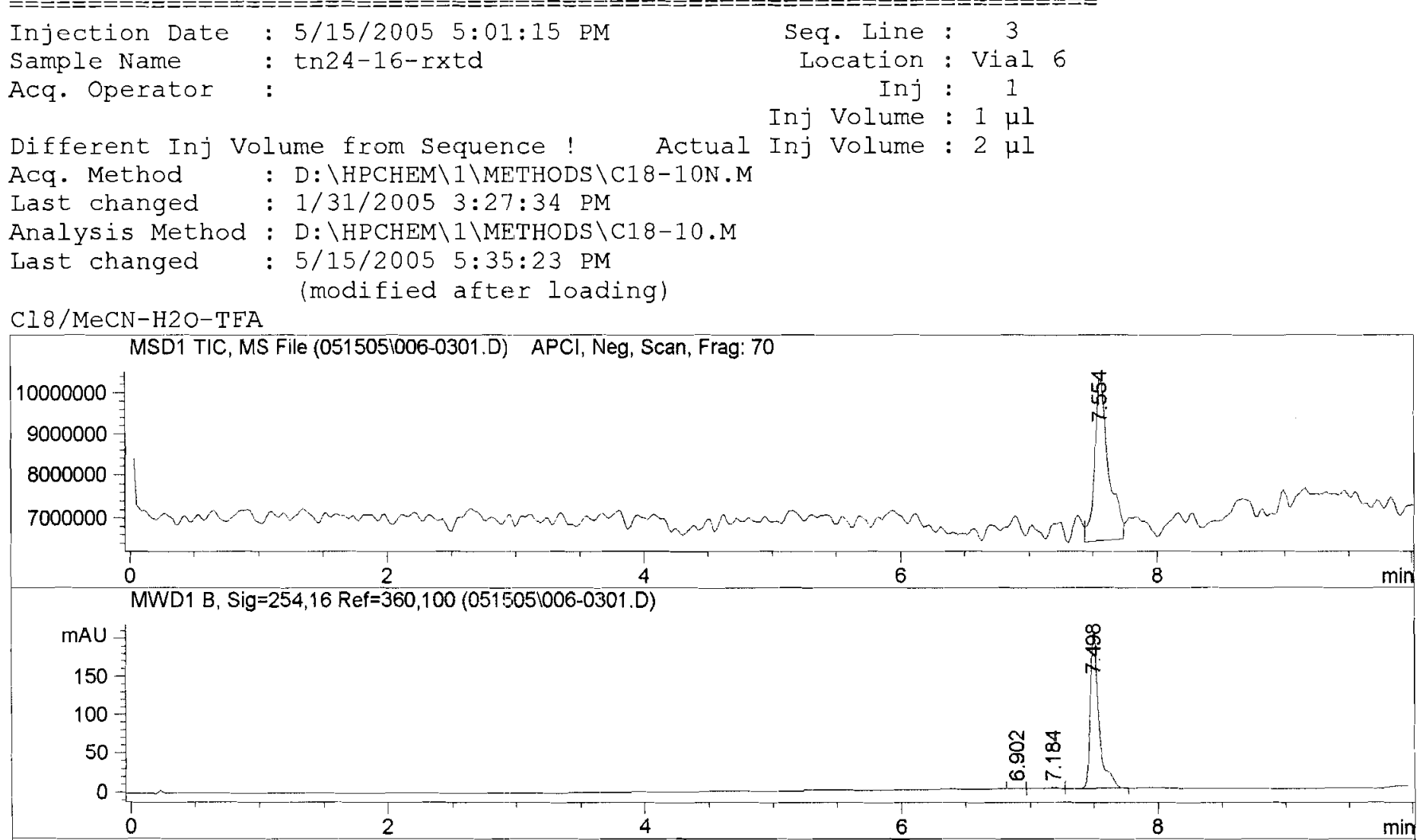
MWD1 C, Sig $=210,8$ Ref $=$ off $(051505100 \overline{6}-0301 . D)$

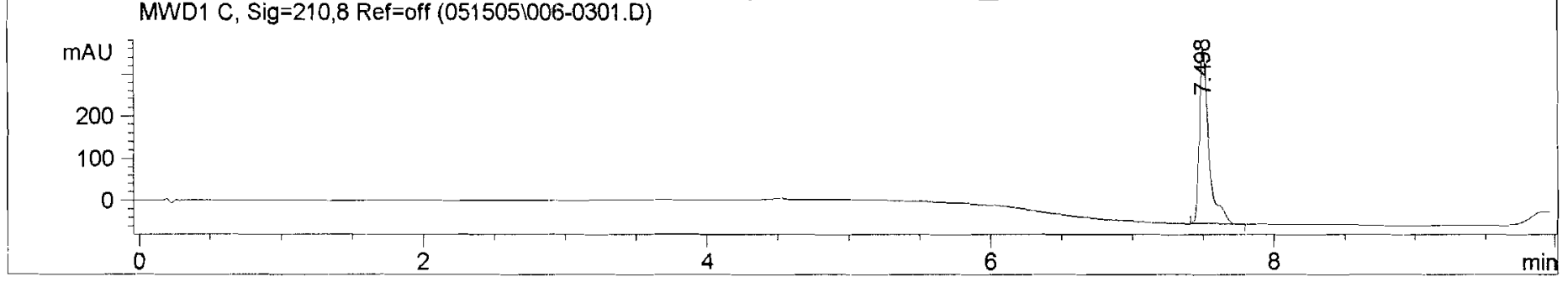

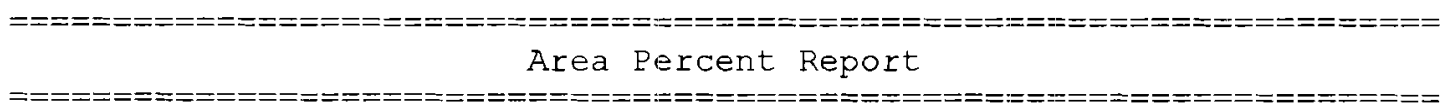

Sorted By

Multiplier

Dilution

\section{: Signal}

$: \quad 1.0000$

$: \quad 1.0000$

Signal 1: MSD1 TIC, MS File

Peak RetTime Type width Area Height Area \# [min] [min]

$\begin{array}{lllllll}1 & 7.554 \mathrm{VV} & 0.1084 & 2.90582 \mathrm{e} & 3.94457 \mathrm{e} 6 & 100.0000\end{array}$

Totals : $\quad 2.90582 \mathrm{e} 7 \quad 3.94457 \mathrm{e} 6$

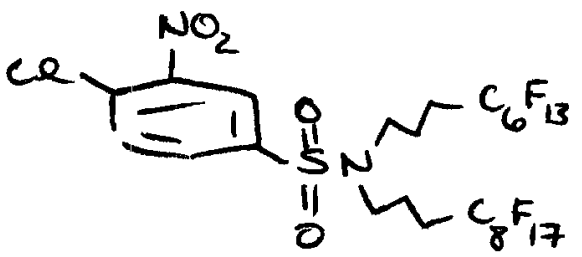


Signal 2: MWD1 B, Sig=254,16 Ref:=360,100

\begin{tabular}{|c|c|c|c|c|c|c|}
\hline $\begin{array}{c}\text { Peak } \\
\#\end{array}$ & $\begin{array}{c}\text { RetTime } \\
\text { [min] }\end{array}$ & Type & $\begin{array}{c}\text { width } \\
\text { [min] }\end{array}$ & $\begin{array}{c}\text { Area } \\
{\left[m A U^{*} s\right]}\end{array}$ & $\begin{array}{l}\text { Height } \\
{[\mathrm{mAU}]}\end{array}$ & $\begin{array}{c}\text { Area } \\
\frac{8}{8}\end{array}$ \\
\hline & & & ------ & $-\cdots---\cdots$ & ---------- & -------1 \\
\hline 1 & 6.902 & BV & 0.0627 & 2.57067 & $6.15600 e^{-1}$ & 0.2697 \\
\hline 2 & 7.184 & VV & 0.0933 & 4.84675 & $7.31570 e^{-1}$ & 0.5084 \\
\hline 3 & 7.498 & VP & 0.0702 & 945.87537 & 203.62404 & 99.2219 \\
\hline Total & : & & & 953.29279 & 204.97121 & \\
\hline
\end{tabular}

Signal 3: MWD1 C, Sig=210,8 Ref=off

\begin{tabular}{|c|c|c|c|c|c|c|}
\hline $\begin{array}{c}\text { Peak } \\
\quad \#\end{array}$ & $\begin{array}{c}\text { RetTime } \\
\text { [min] }\end{array}$ & Type & $\begin{array}{l}\text { width } \\
\text { [min] }\end{array}$ & $\begin{array}{c}\text { Area } \\
{\left[\mathrm{mAU}^{*} \mathrm{~s}\right]}\end{array}$ & $\begin{array}{l}\text { Height } \\
\text { [mAU] }\end{array}$ & $\begin{array}{c}\text { Area } \\
8\end{array}$ \\
\hline---1 & ------- & & -------1 & $-----\cdots---$ & -------- & -------- \\
\hline 1 & 7.498 & $\mathrm{BP}$ & 0.0703 & 1943.82935 & 417.98306 & 100.0000 \\
\hline Total & s : & & & 1943.82935 & 417.98306 & \\
\hline
\end{tabular}

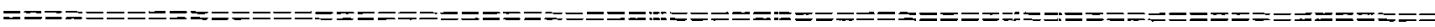


Print of window 80: Apex Mass Spectrum of Peak 7.554 of 006-0301.D $\mathrm{Rf} 6+\mathrm{Rf} 8 / \mathrm{Cl}-\mathrm{NO} 2 /$ in $\mathrm{MeOH}$

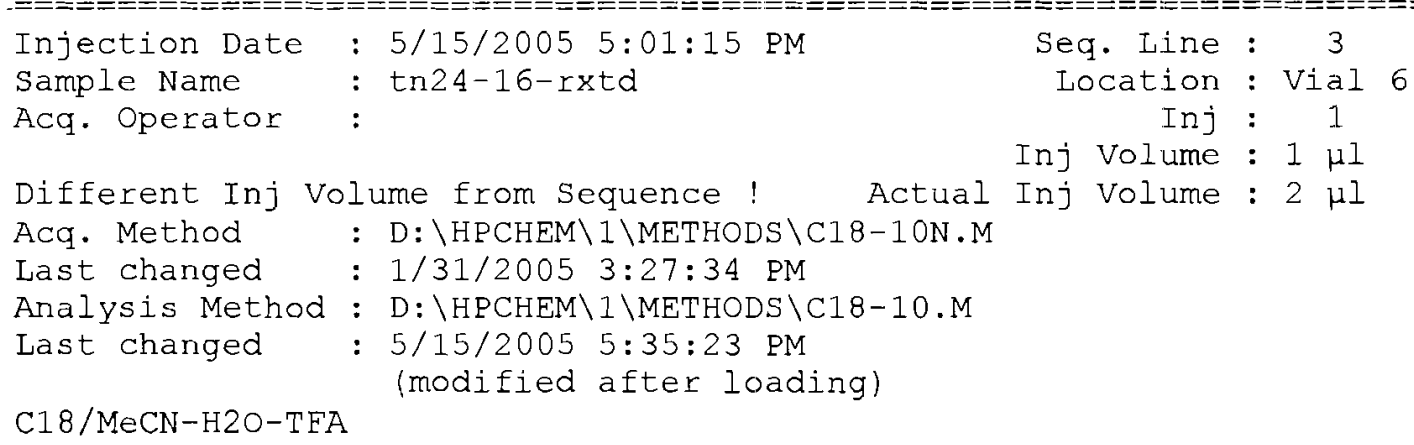

Apex Mass Spectrum of Peak 7.554 of 006-0301.D

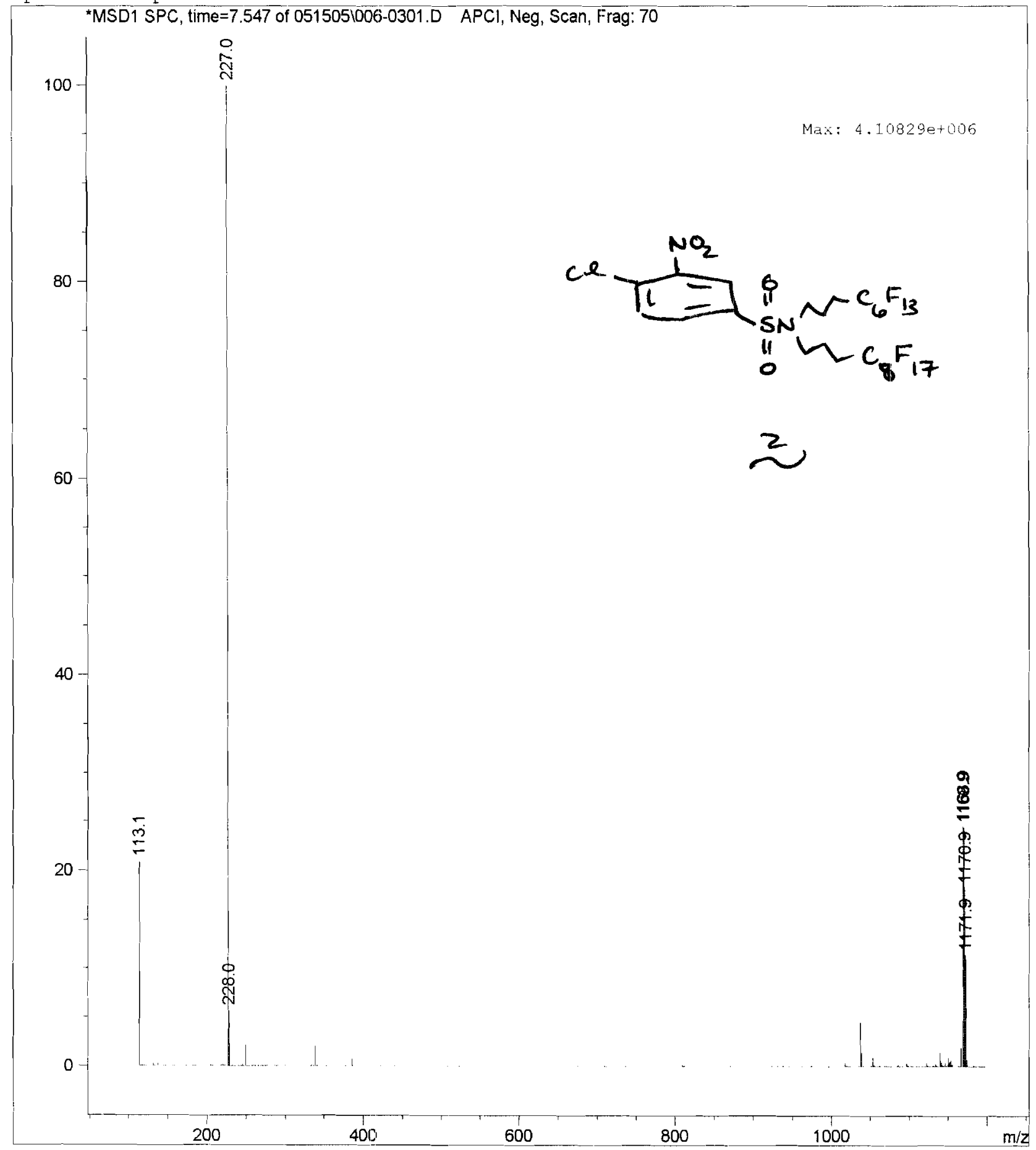




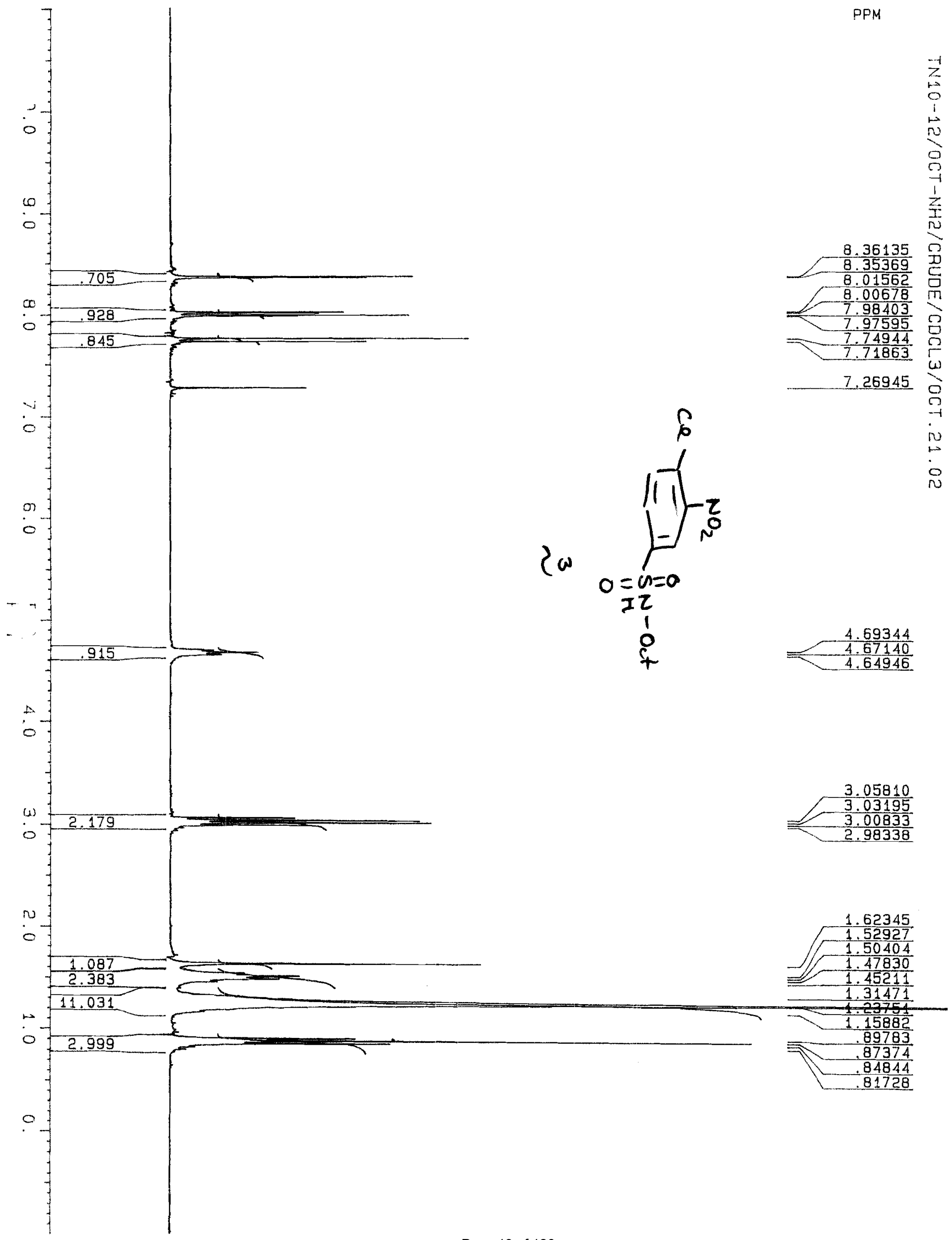

Page 13 of 126 

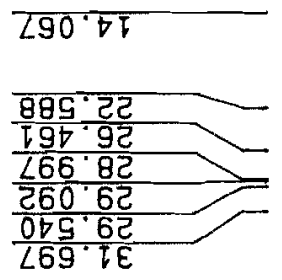

$\overline{6 E} \cdot \mathrm{E}$

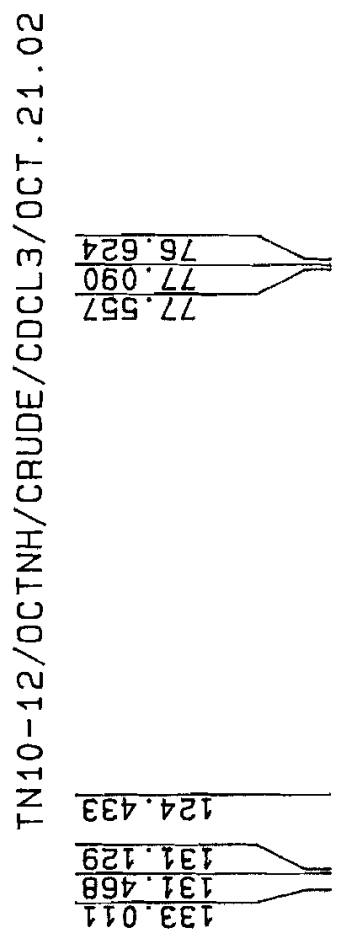

8QE. ODT

टट० $\angle D T$
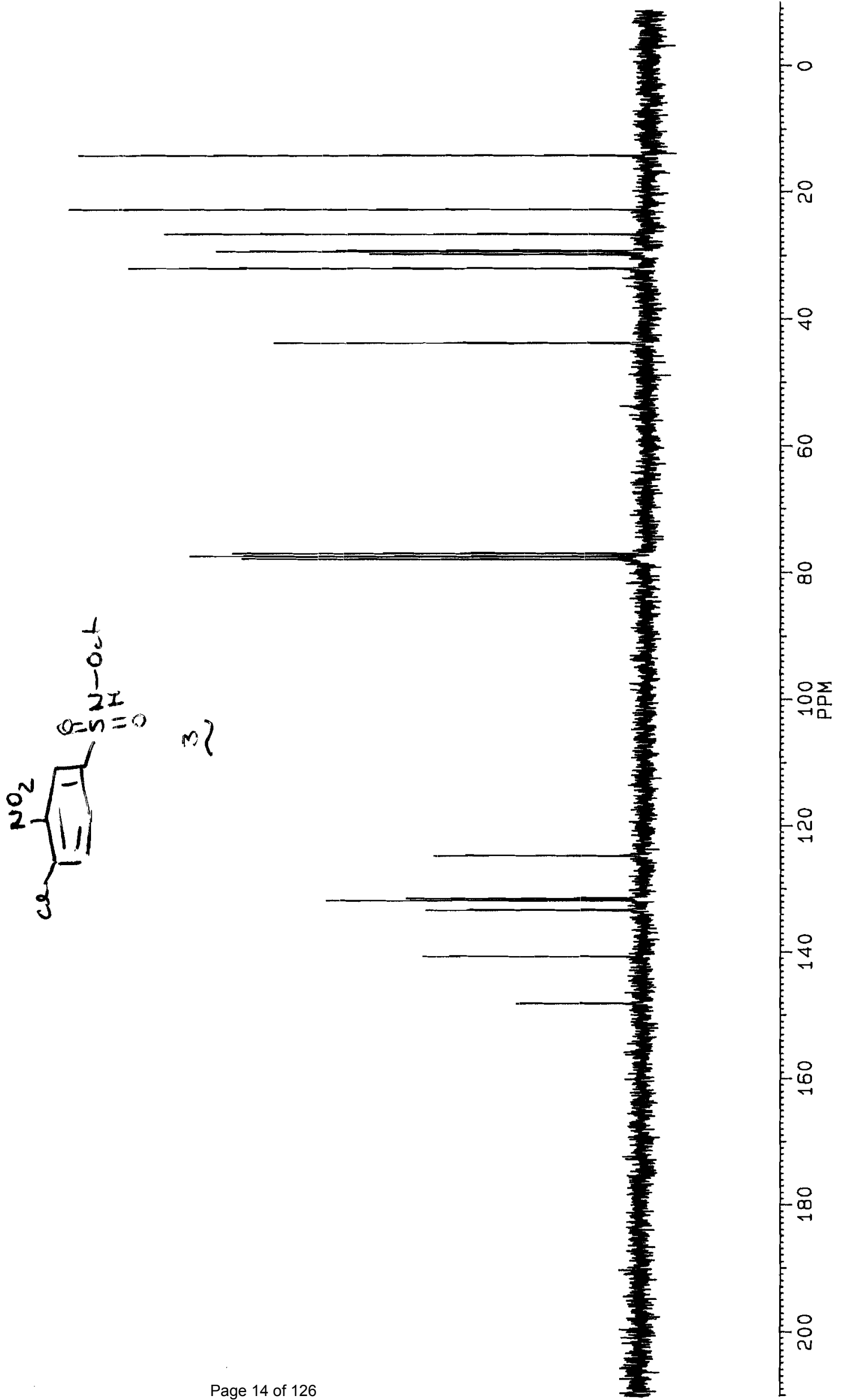


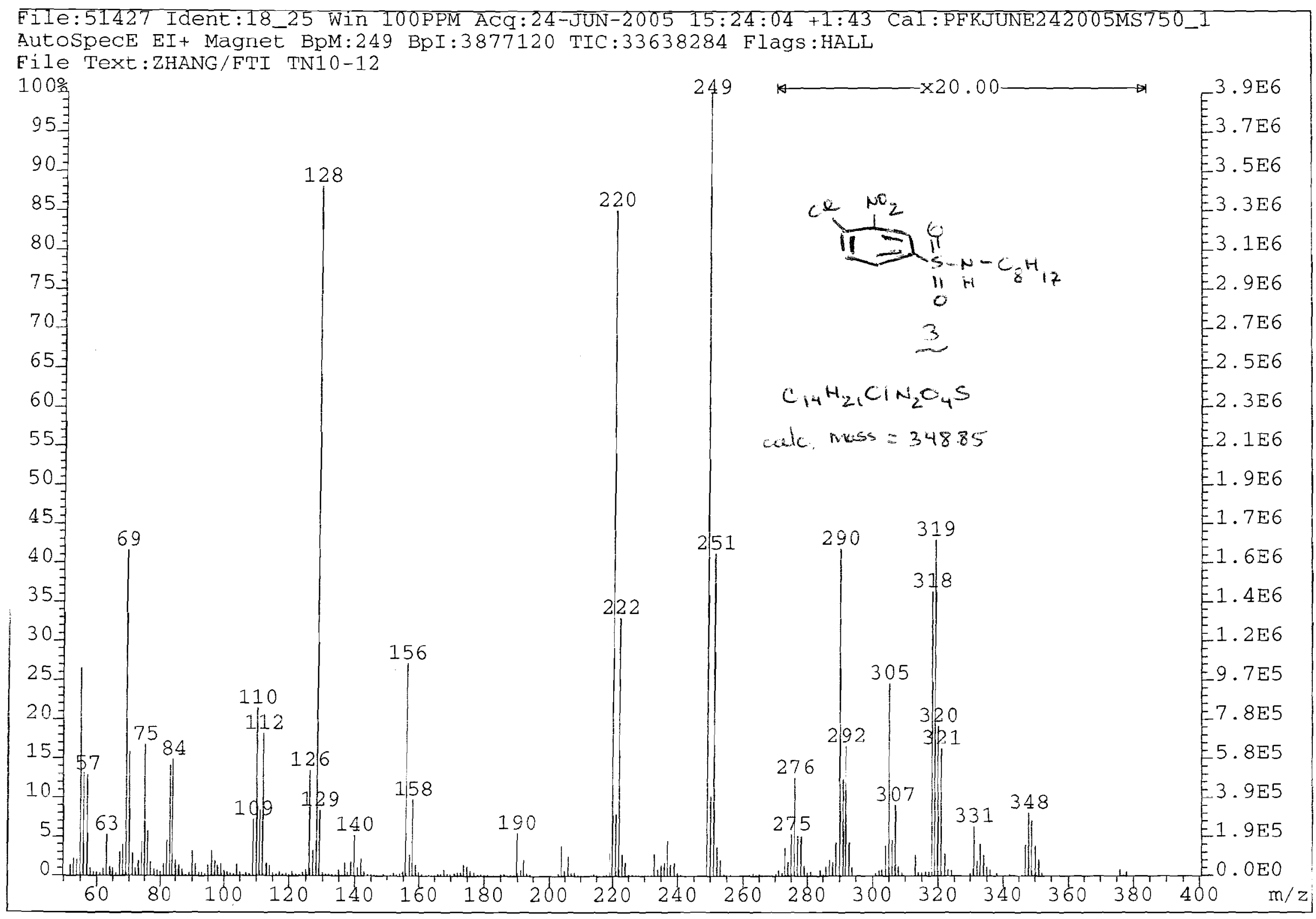

Page 15 of 126 
mono-octyl/ $\mathrm{Cl}-\mathrm{NO} 2-/$ in $\mathrm{MeOH}$

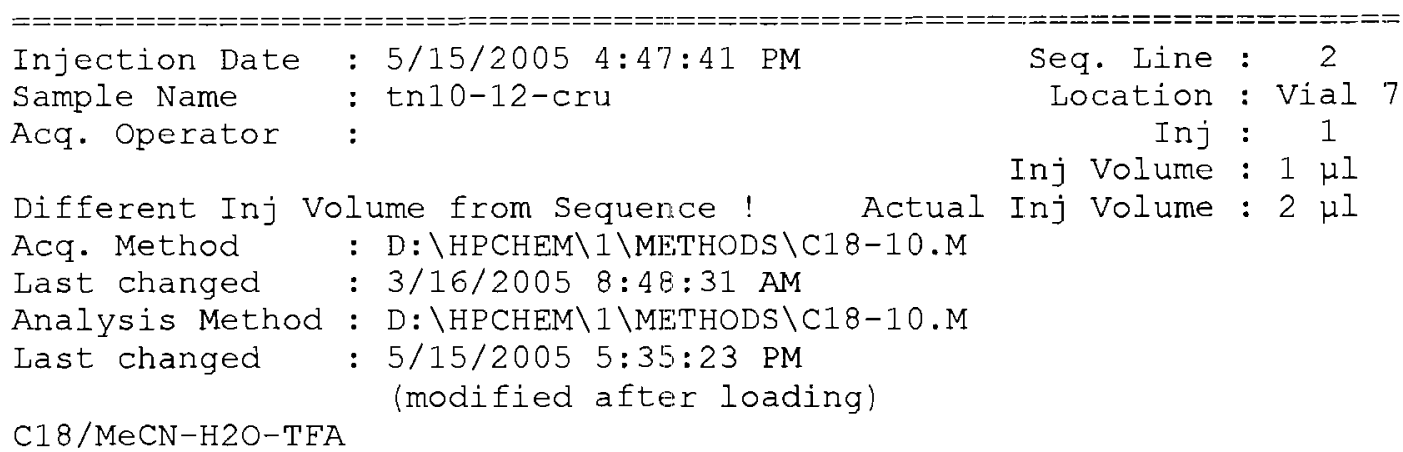

$\mathrm{C} 18 / \mathrm{MeCN}-\mathrm{H} 2 \mathrm{O}-\mathrm{TEA}$

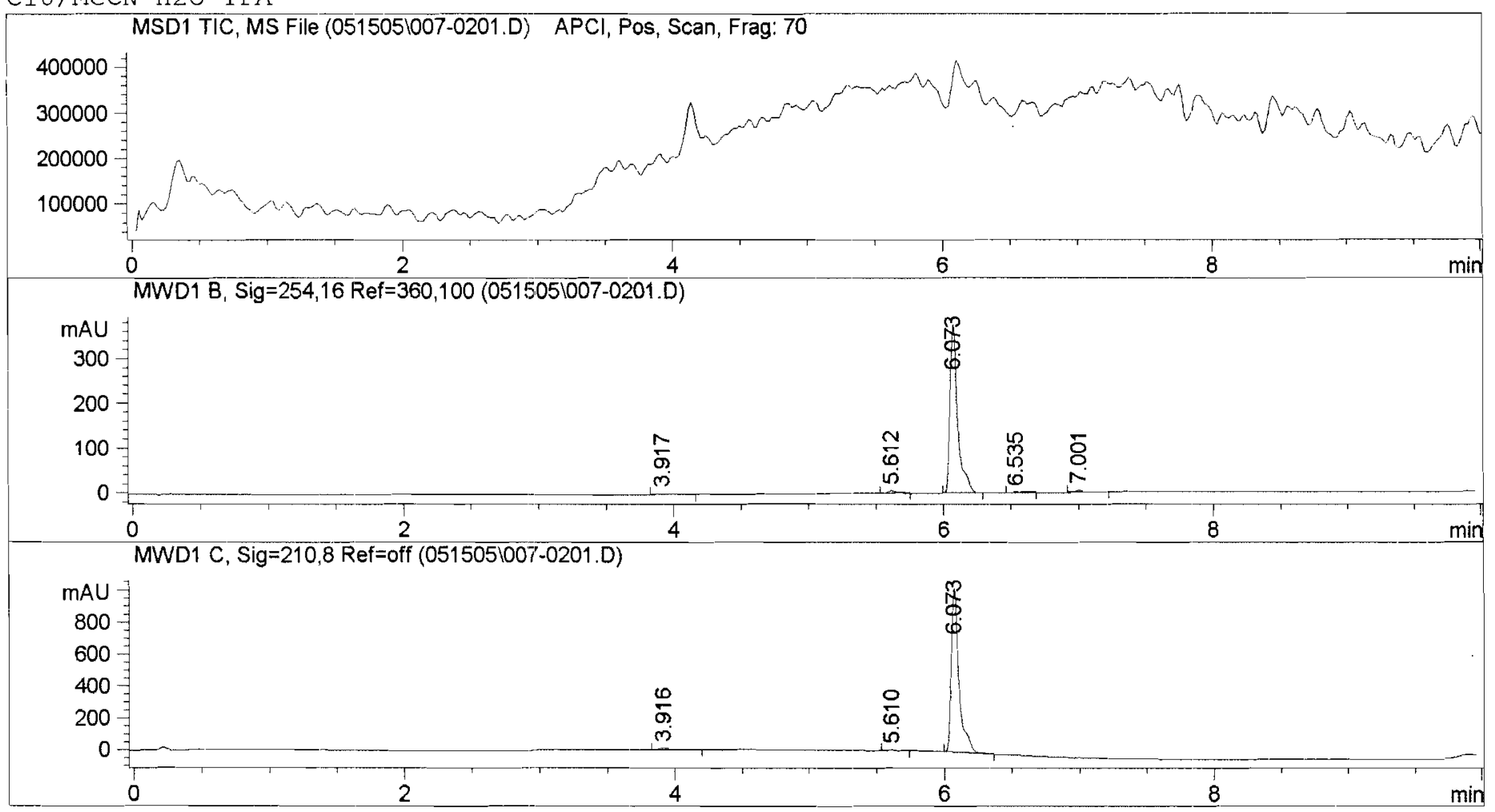

Area Percent Report

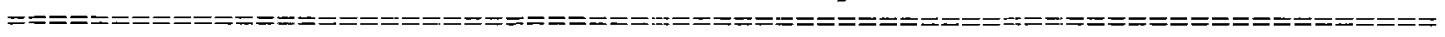

$\begin{array}{lll}\text { Sorted BY } & : & \text { Signal } \\ \text { Multiplier } & : & 1.0000 \\ \text { Dilution } & : & 1.0000\end{array}$

Dilution

1.0000

1.0000

Signal 1: MSDl TIC, MS File

Signal 2: MWD1 B, Sig=254,16 Ref:=360, 100

\begin{tabular}{|c|c|c|c|c|c|c|}
\hline $\begin{array}{c}\text { Peak } \\
\#\end{array}$ & $\begin{array}{l}\text { RetTime } \\
\text { [min] }\end{array}$ & Type & $\begin{array}{c}\text { Width } \\
\text { [min] }\end{array}$ & $\begin{array}{c}\text { Area } \\
{\left[\mathrm{mAU}{ }^{*} \mathrm{~s}\right]}\end{array}$ & $\begin{array}{l}\text { Height } \\
\text { [mAU] }\end{array}$ & $\begin{array}{c}\text { Area } \\
\frac{8}{8}\end{array}$ \\
\hline & & & & & & \\
\hline 1 & 3.917 & $\mathrm{~PB}$ & 0.0860 & 13.75425 & 2.29146 & 0.8969 \\
\hline 2 & 5.612 & $\mathrm{BB}$ & 0.0608 & 22.18532 & 5.52875 & 1.4467 \\
\hline 3 & 6.073 & $\mathrm{BB}$ & 0.0579 & 1471.06714 & 373.54904 & 95.9260 \\
\hline 4 & 6.535 & $\mathrm{~PB}$ & 0.0750 & 4.79750 & $8.88274 e^{-1}$ & 0.3128 \\
\hline 5 & 7.001 & $\mathrm{BB}$ & 0.0671 & 21.73923 & 4.77502 & 1.4176 \\
\hline
\end{tabular}

Totals :

$1533.54343 \quad 387.03255$

Page 16 of 126 
Signal 3: MWD1 C, Sig=210,8 Ref=off

\begin{tabular}{|c|c|c|c|c|c|c|}
\hline $\begin{array}{c}\text { Peak } \\
\#\end{array}$ & $\begin{array}{c}\text { Retrime } \\
\text { [min] }\end{array}$ & Type & $\begin{array}{l}\text { Width } \\
\text { [min] }\end{array}$ & $\begin{array}{c}\text { Area } \\
{\left[\mathrm{mAU}^{\star} \mathrm{s}\right]}\end{array}$ & $\begin{array}{l}\text { Height } \\
\text { [mAU] }\end{array}$ & $\begin{array}{c}\text { Area } \\
\frac{8}{8}\end{array}$ \\
\hline-- & ------- & & ------- & $-----\cdots---$ & ----------- & -------1 \\
\hline 1 & 3.916 & $\mathrm{PP}$ & 0.0823 & 54.07493 & 9.51558 & 1.2857 \\
\hline 2 & 5.610 & $\mathrm{BB}$ & 0.1846 & 96.10638 & 6.57210 & 2.2850 \\
\hline 3 & 6.073 & $\mathrm{BB}$ & 0.0582 & 4055.71924 & 1024.16699 & 96.4293 \\
\hline Total & : & & & 4205.90054 & 1040.25467 & \\
\hline
\end{tabular}

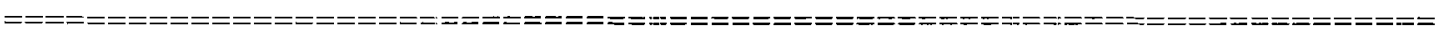

$\star \star \star$ End of Report $\star \star \star ~$ 

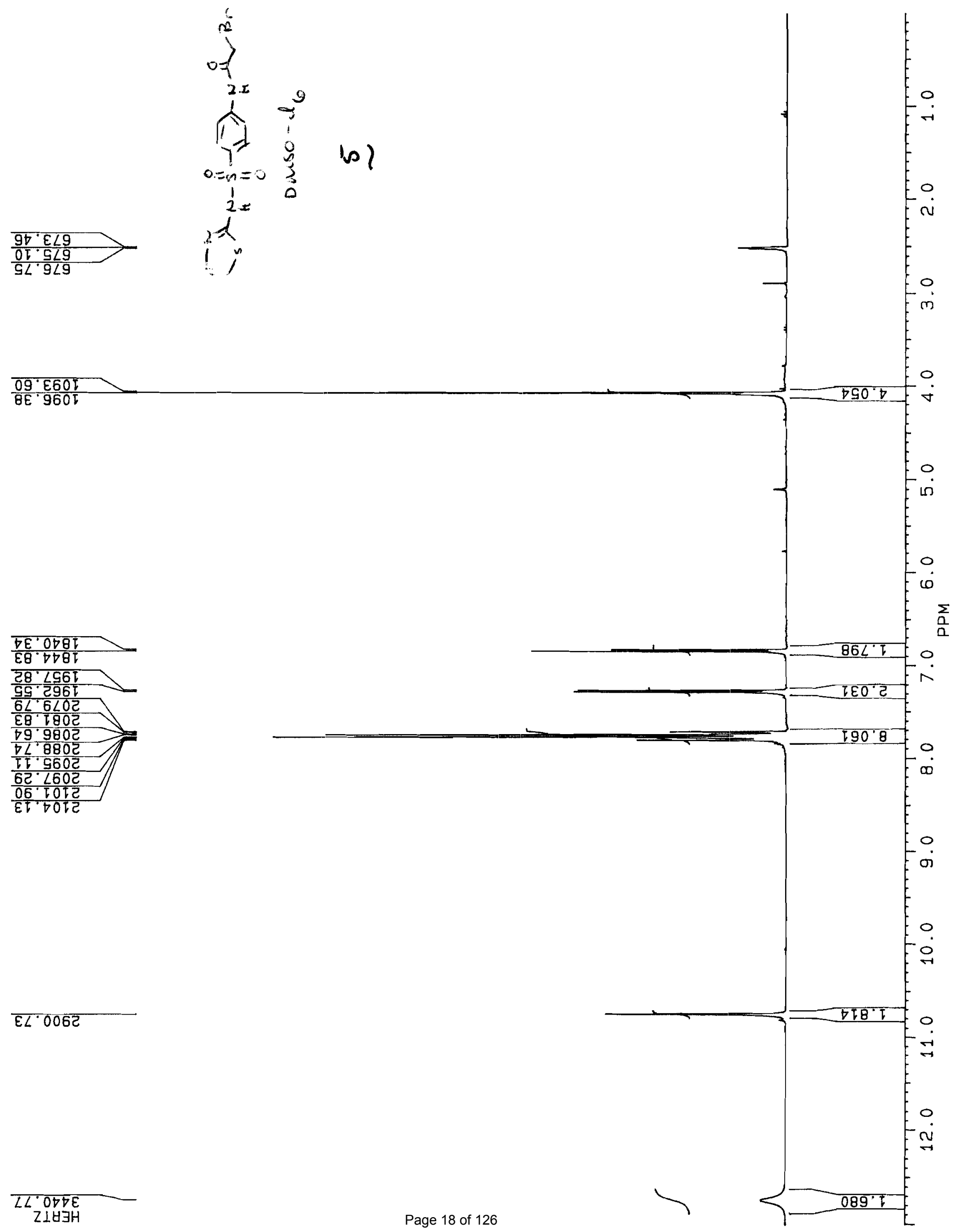


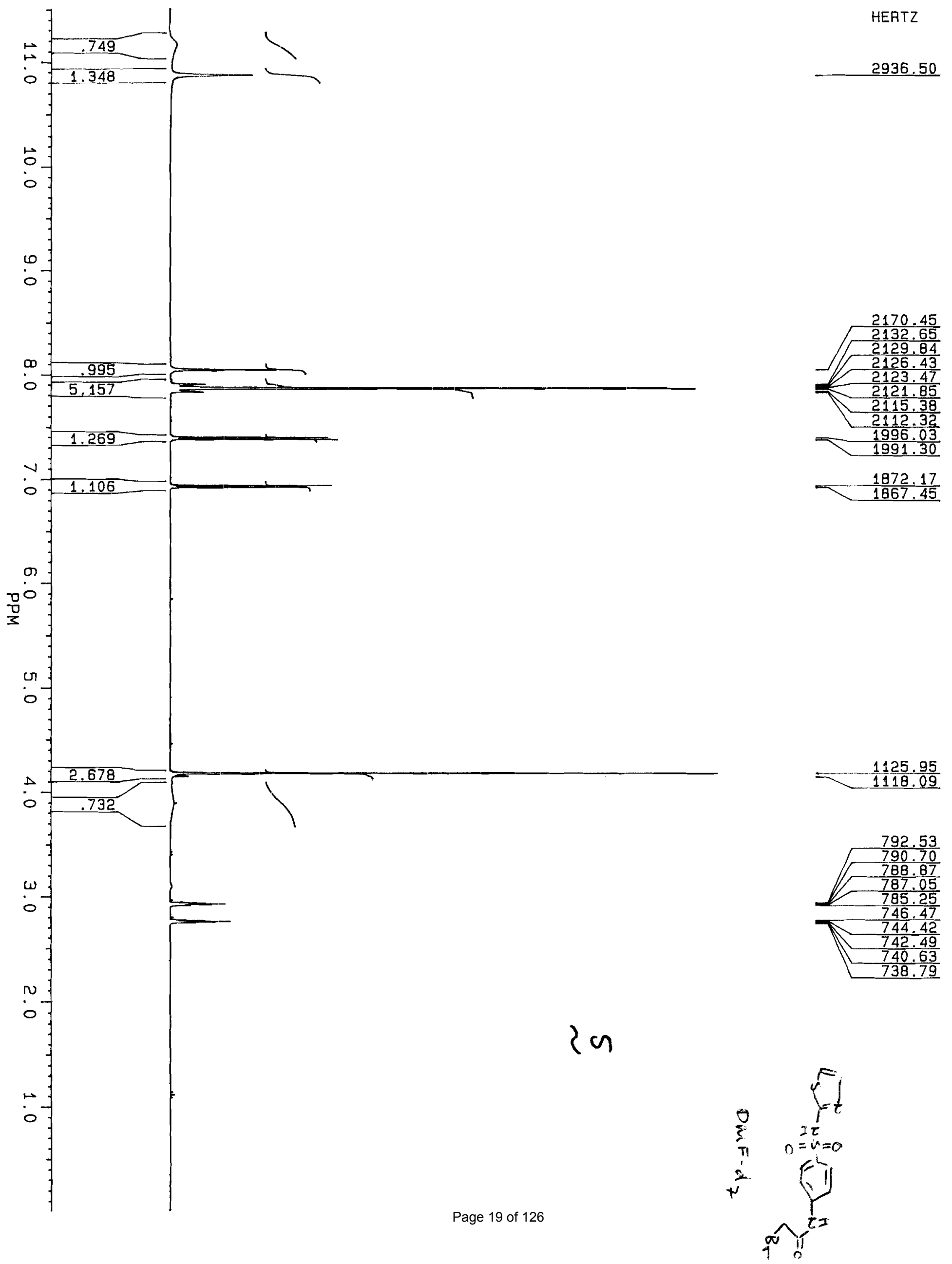



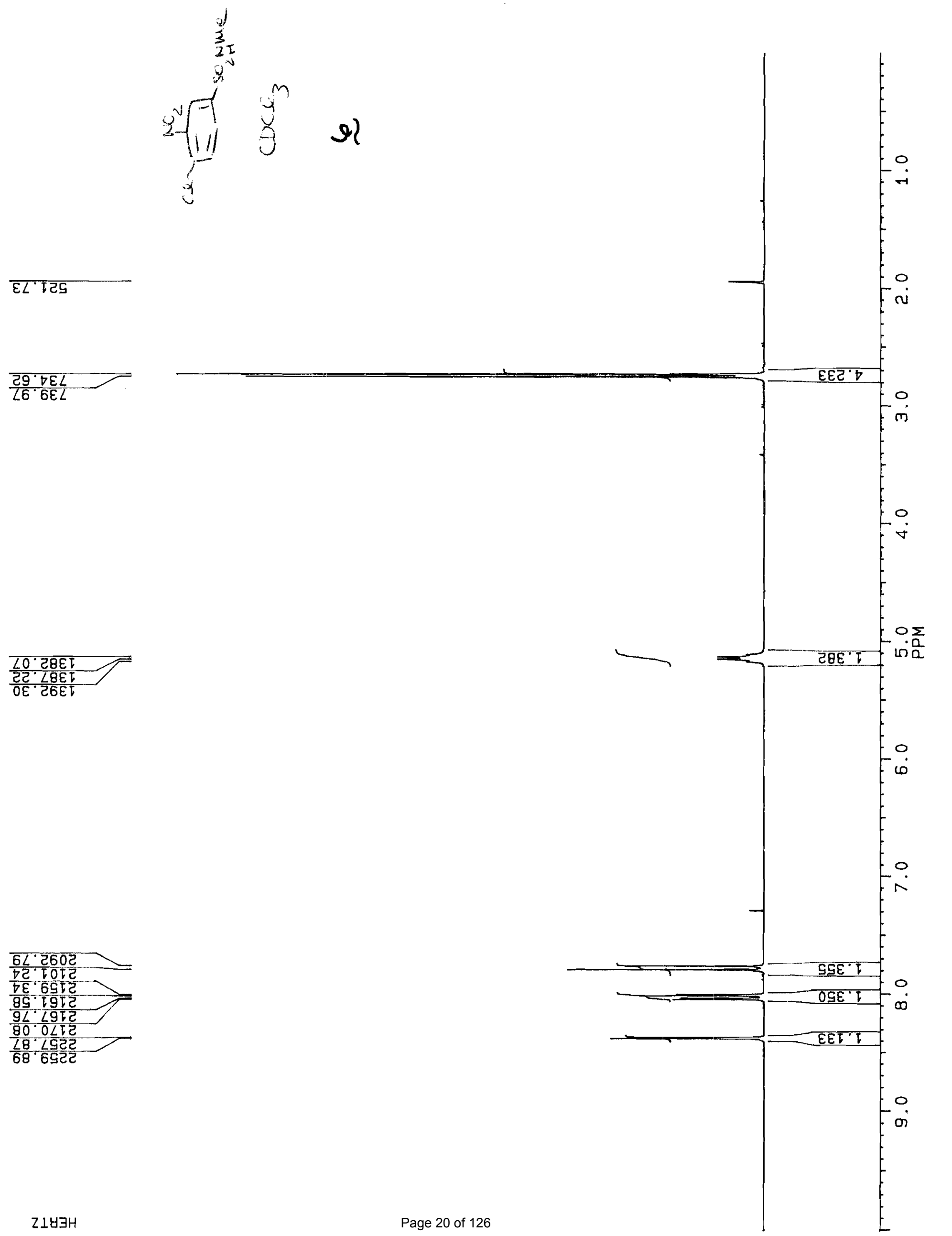


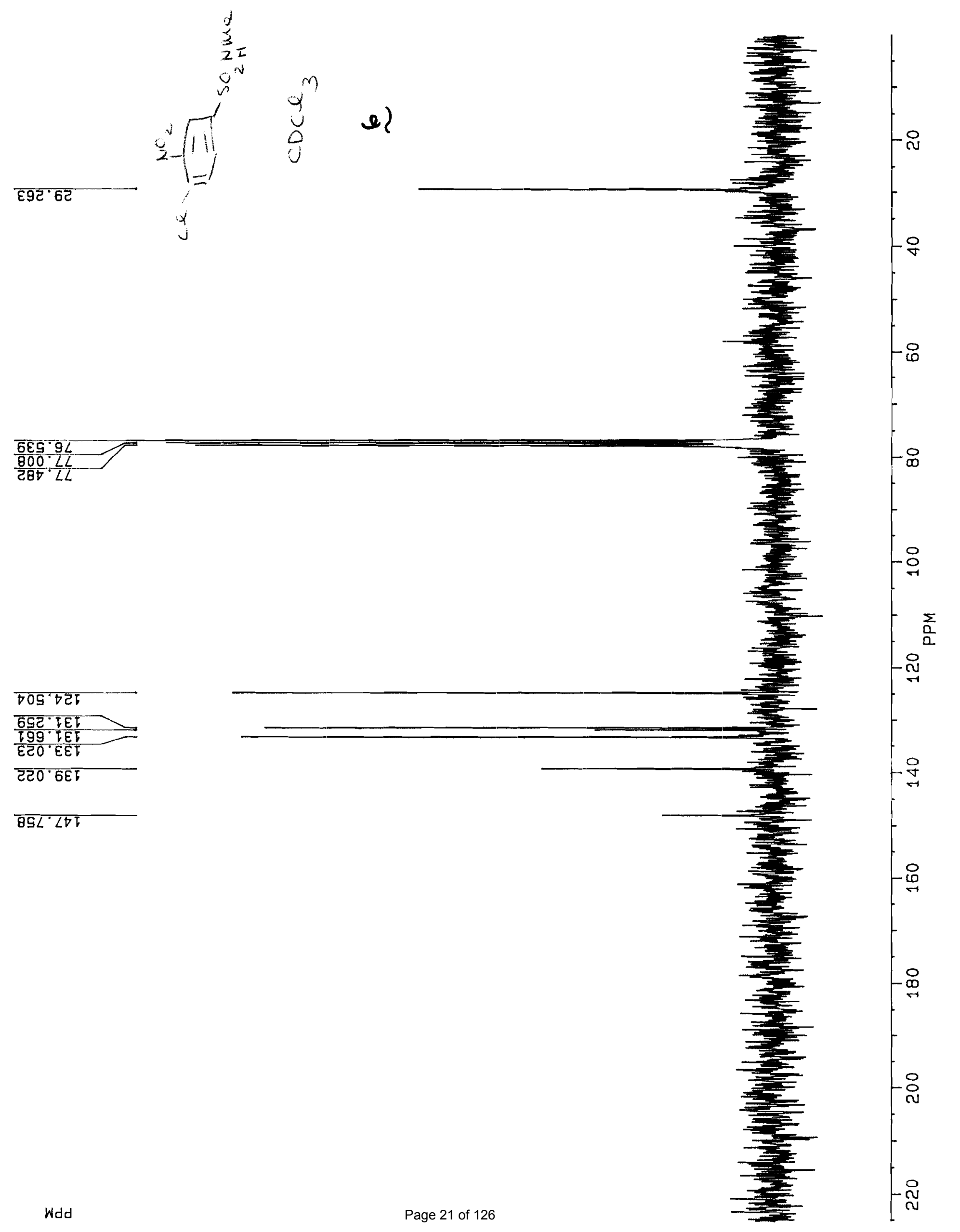




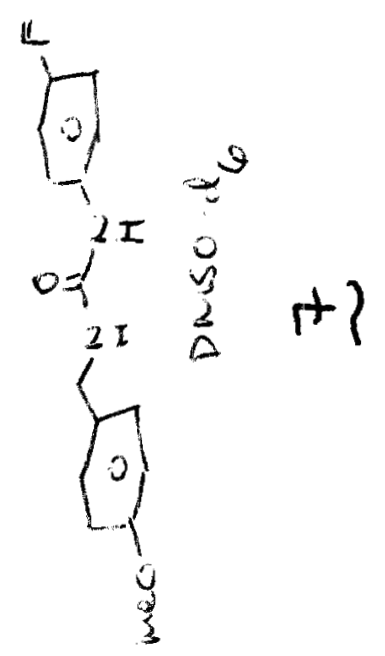

$\overline{56 \cdot S \angle 9}$
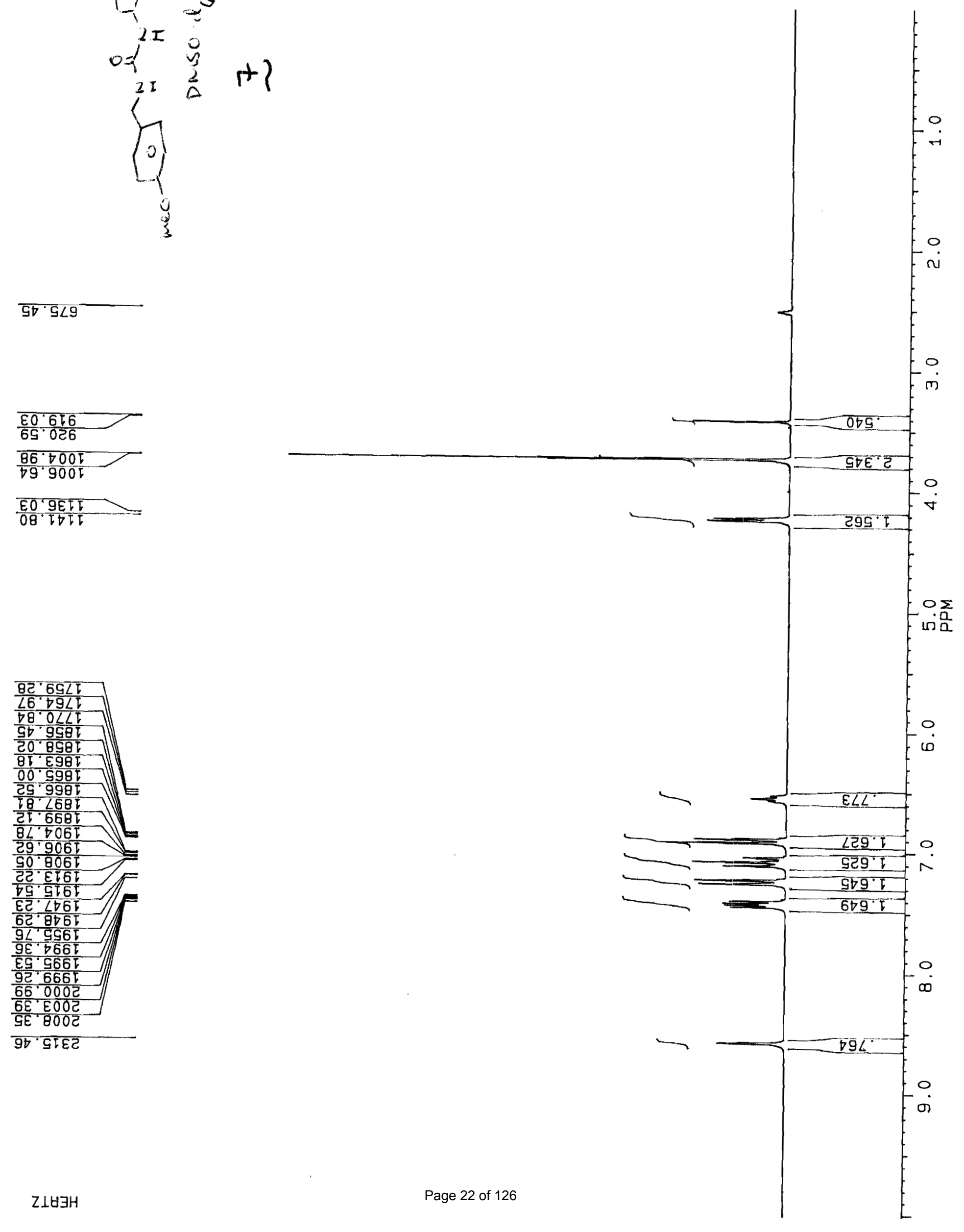


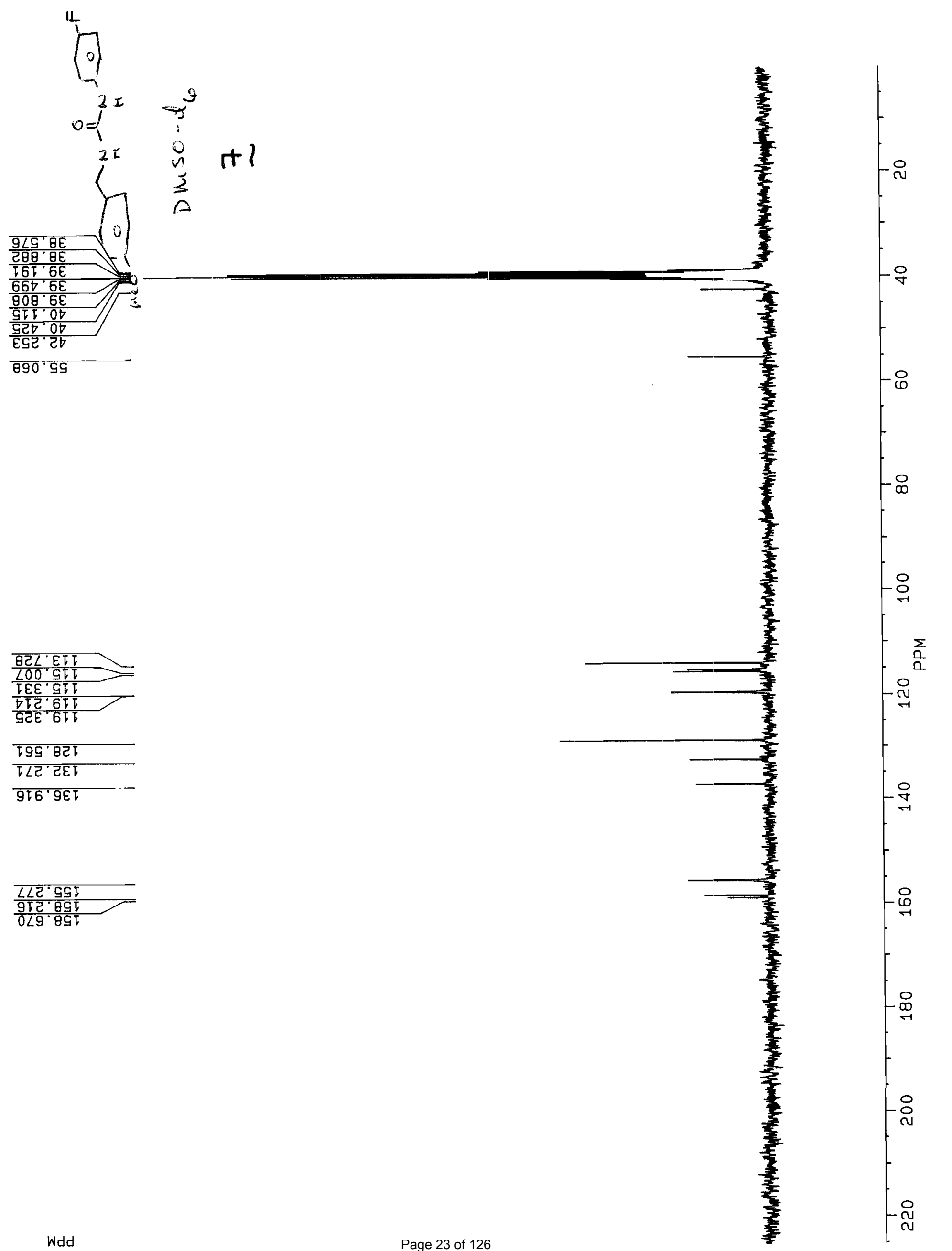



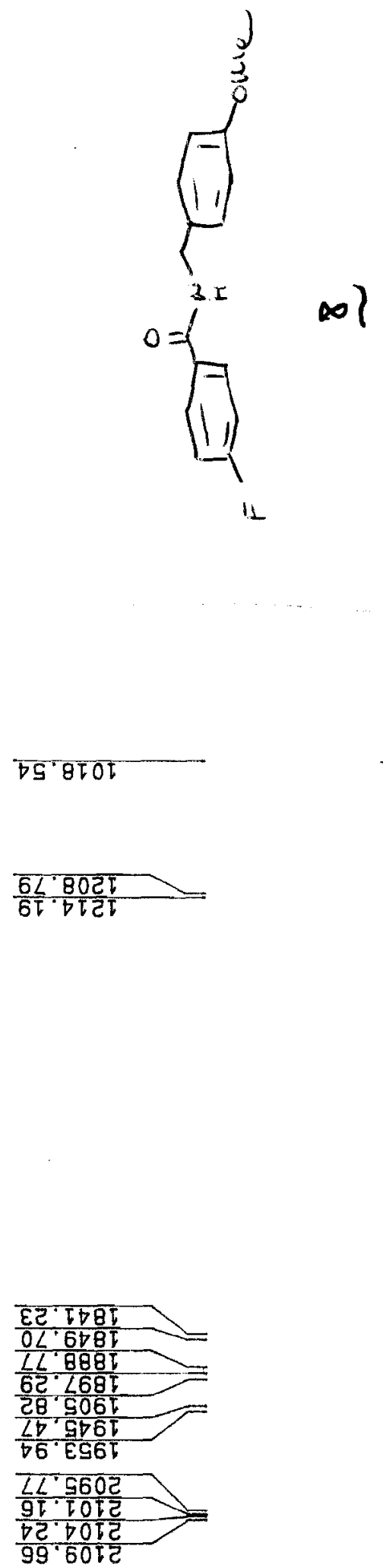


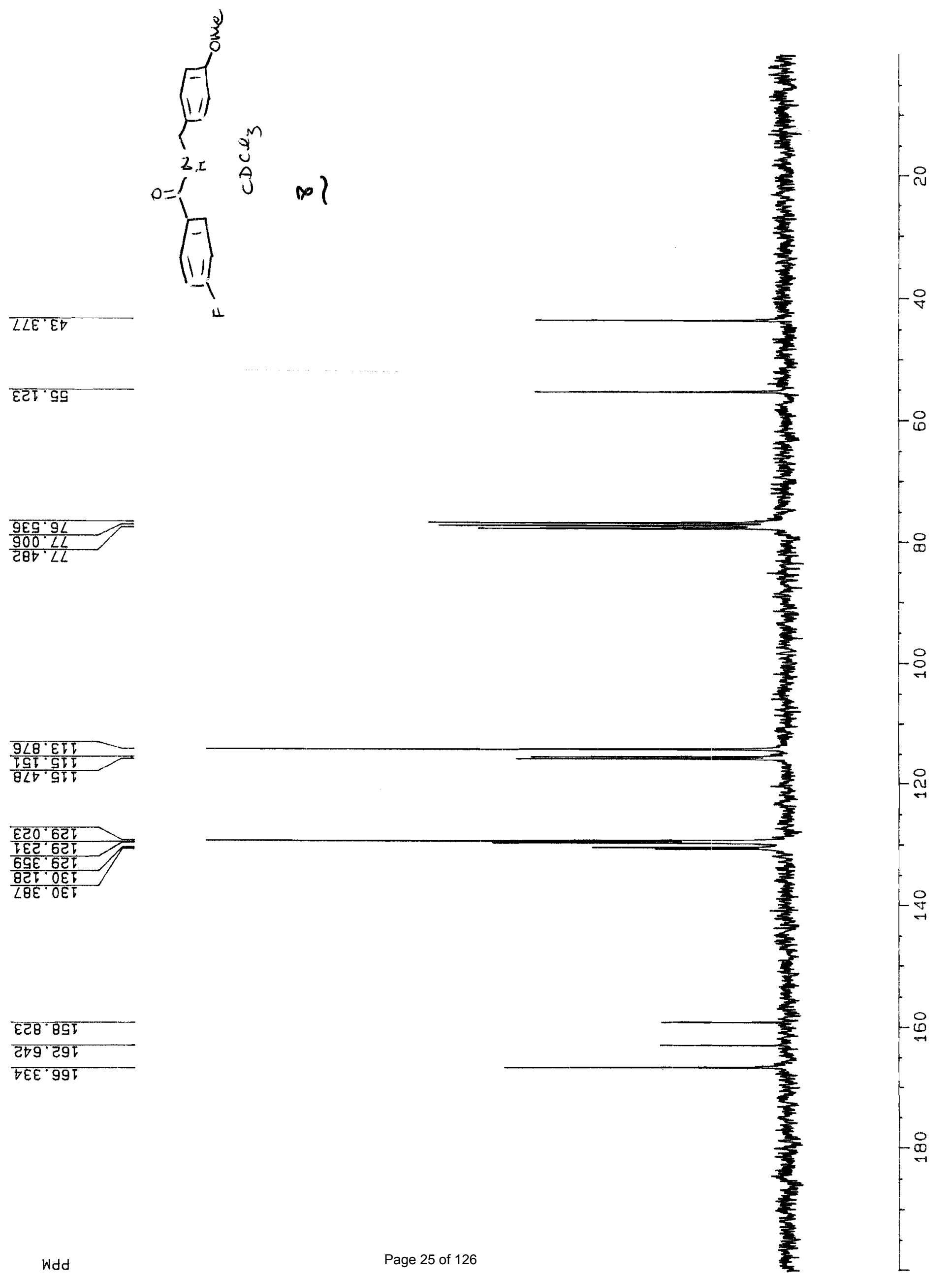




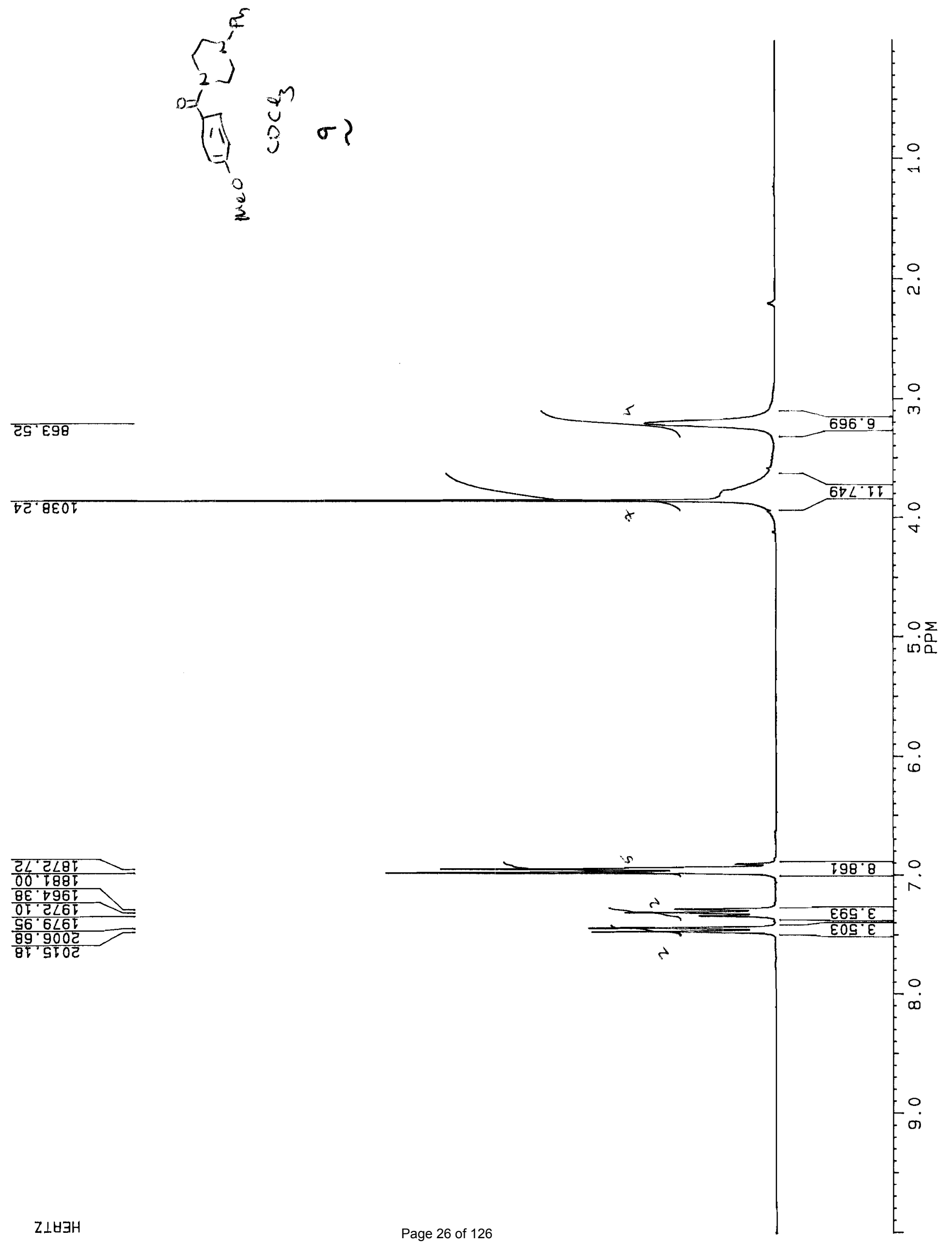




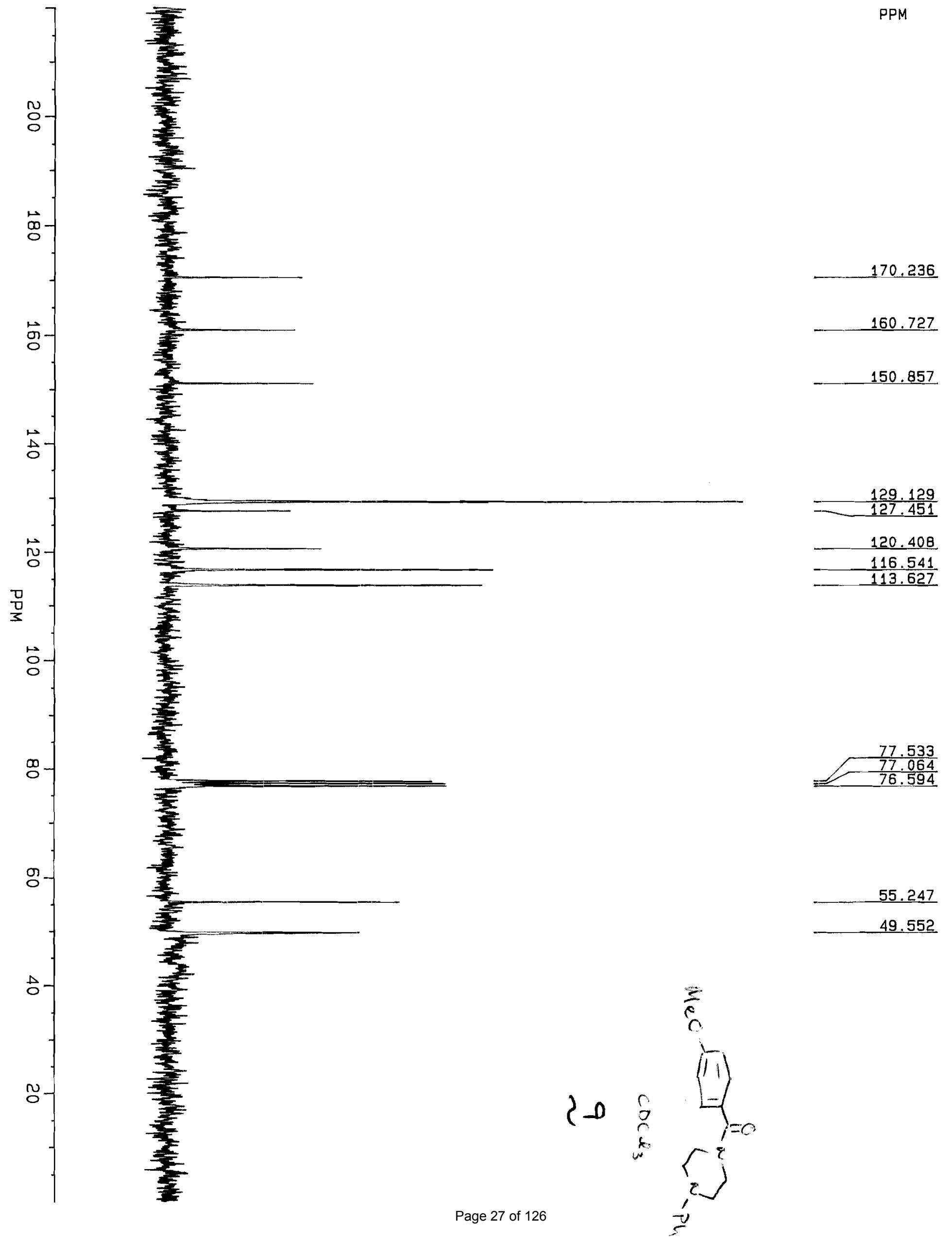




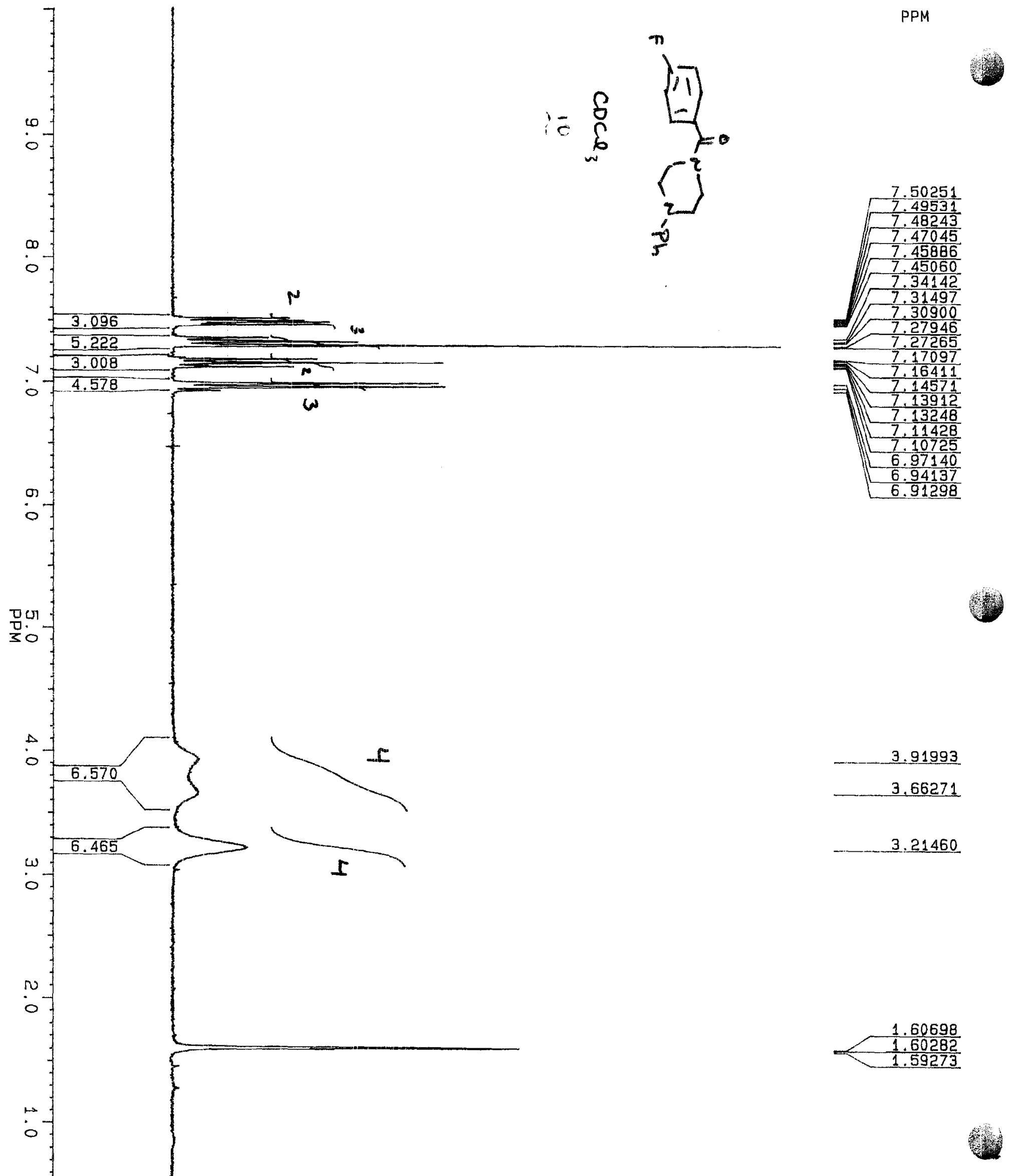




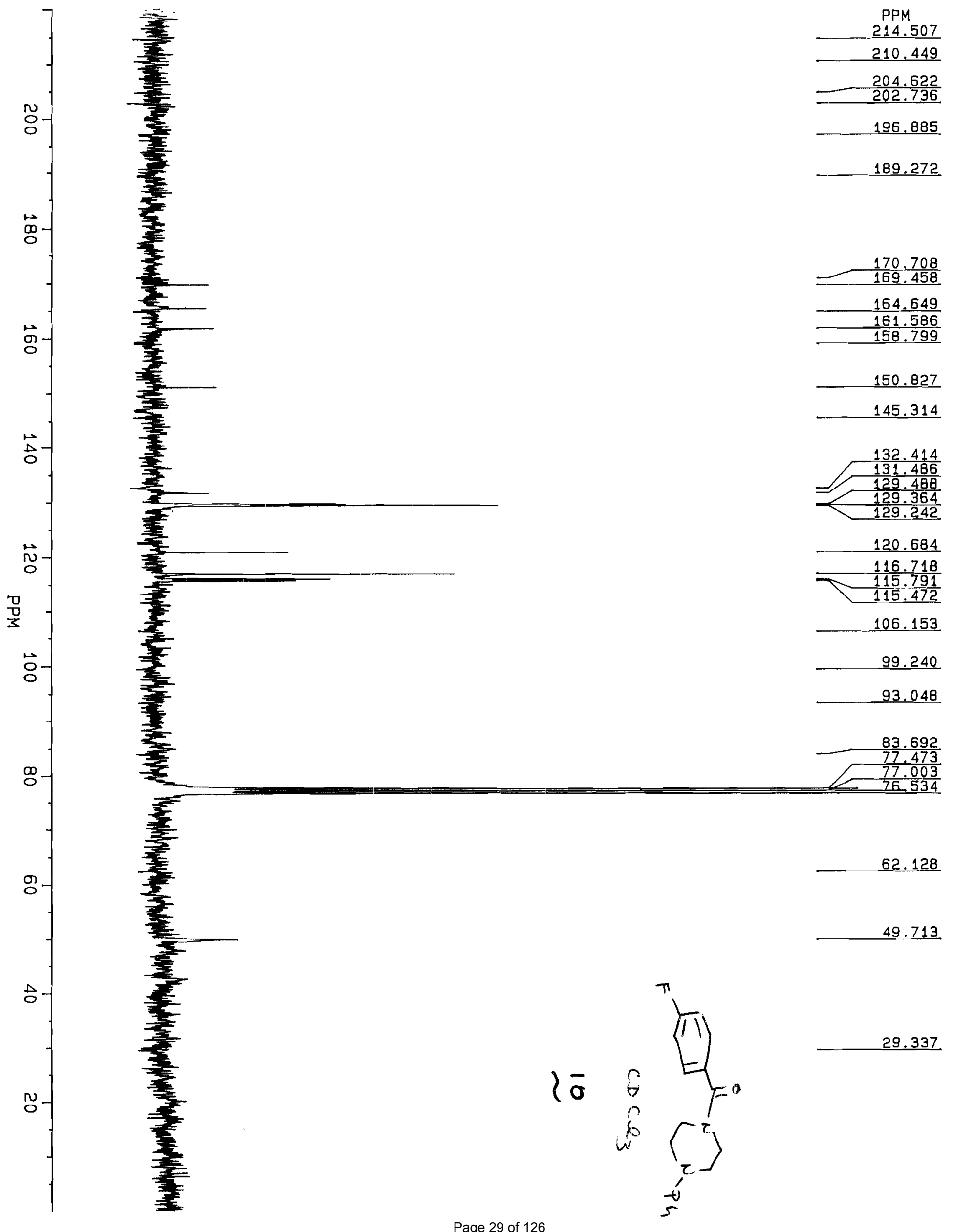




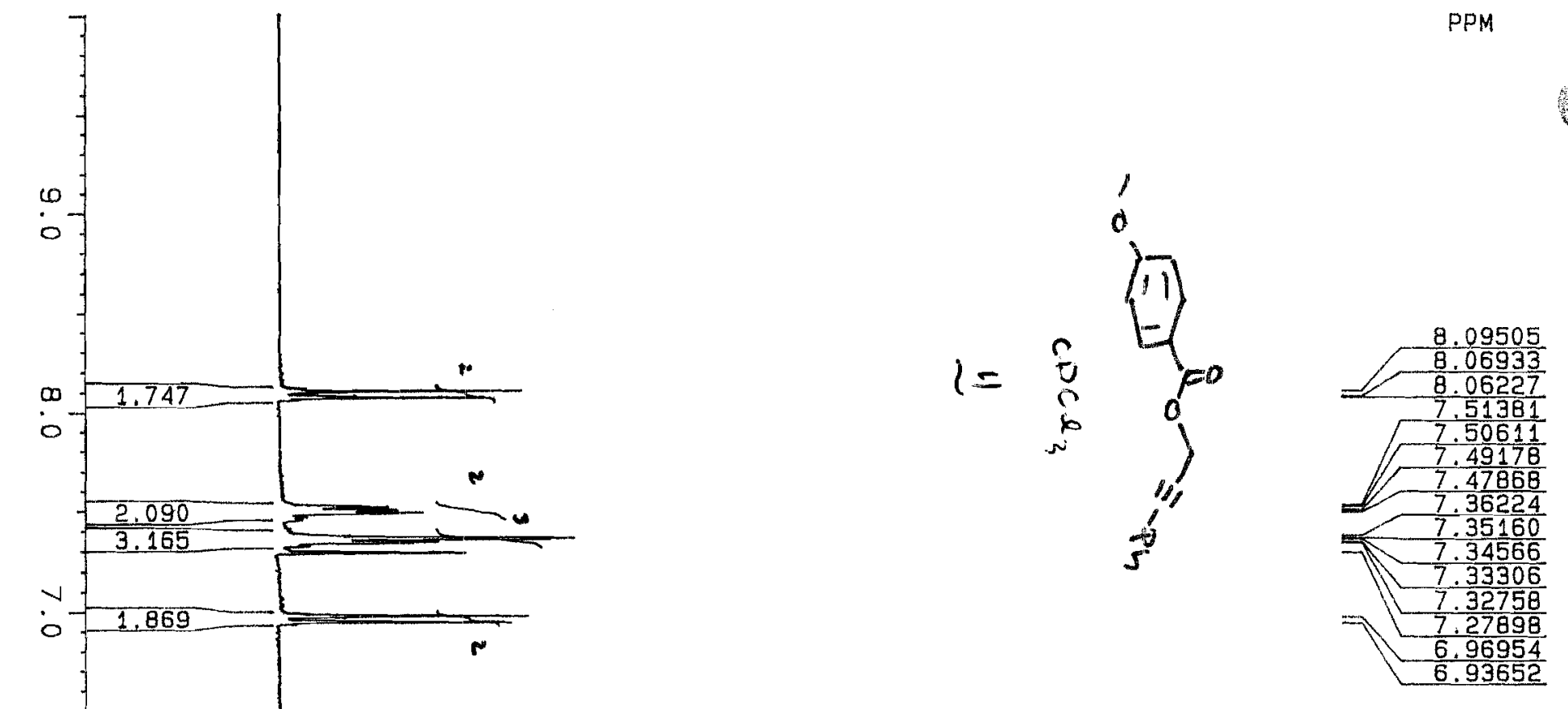

5.14686

3.80808

?

$\omega$

पूर

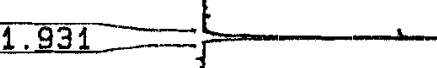

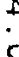
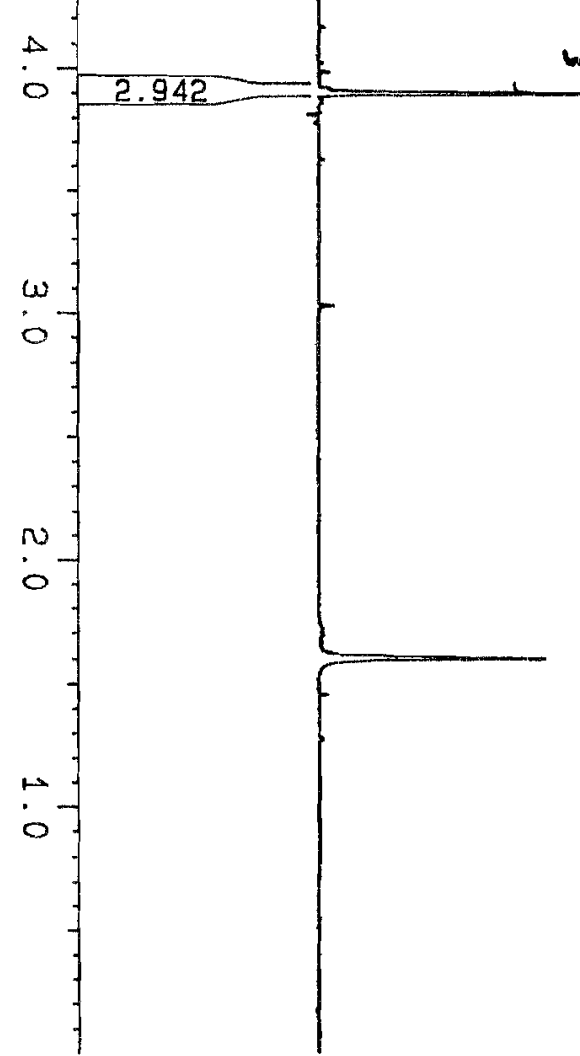

1.59784

Page 30 of 126 


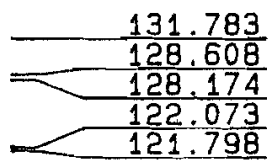

$\stackrel{\circ}{0}$

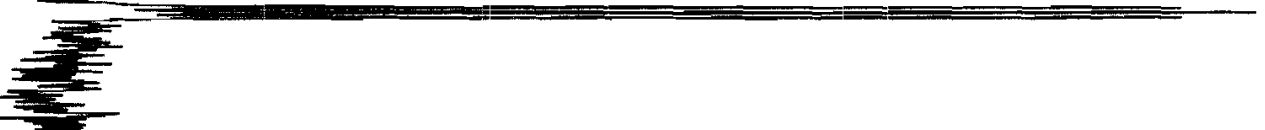

g
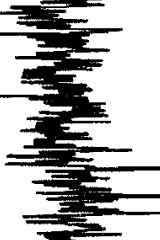

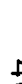

o

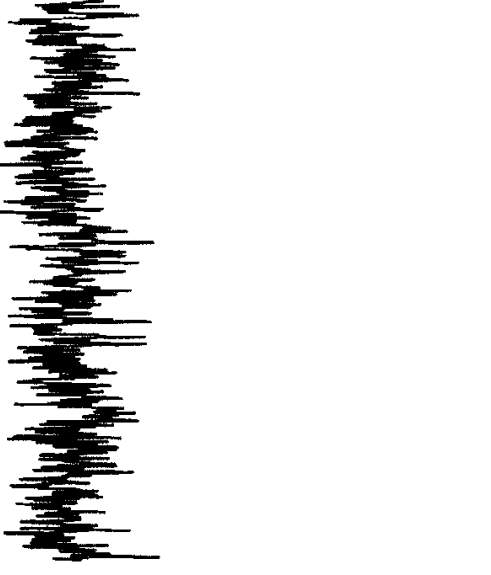

尺
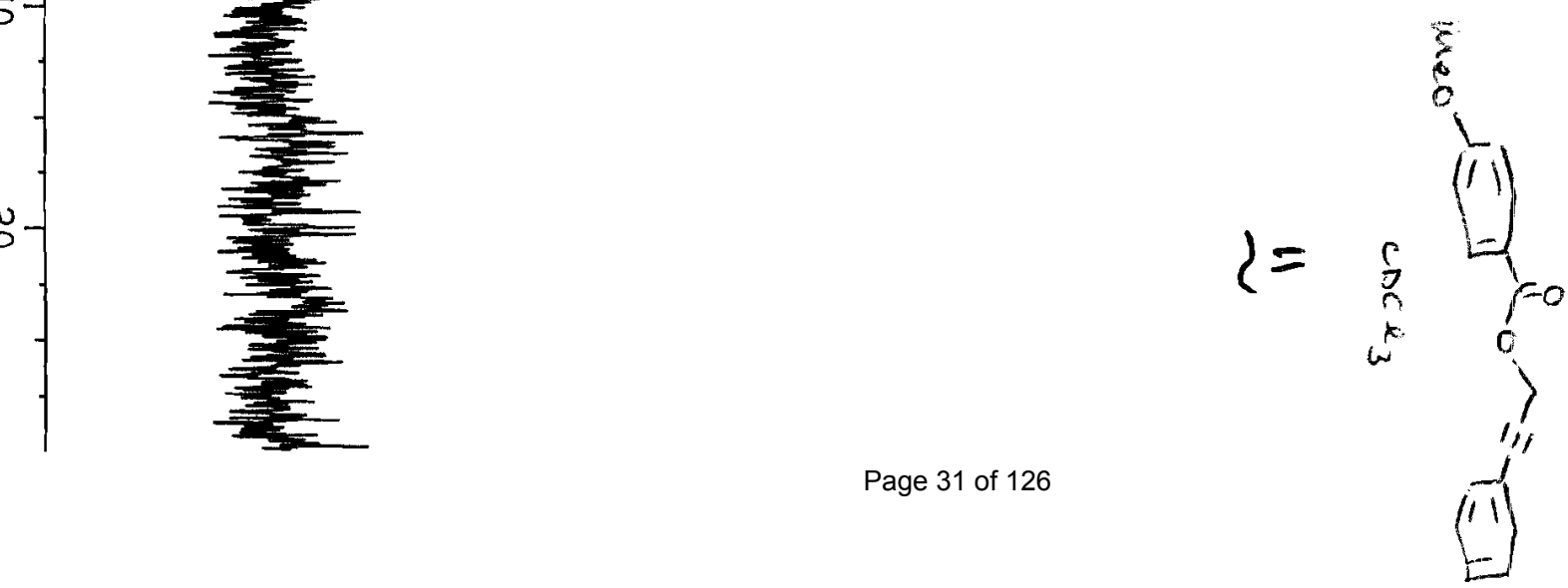

86.260

83.165

77.402

76.933

76.464

Page 31 of 126 


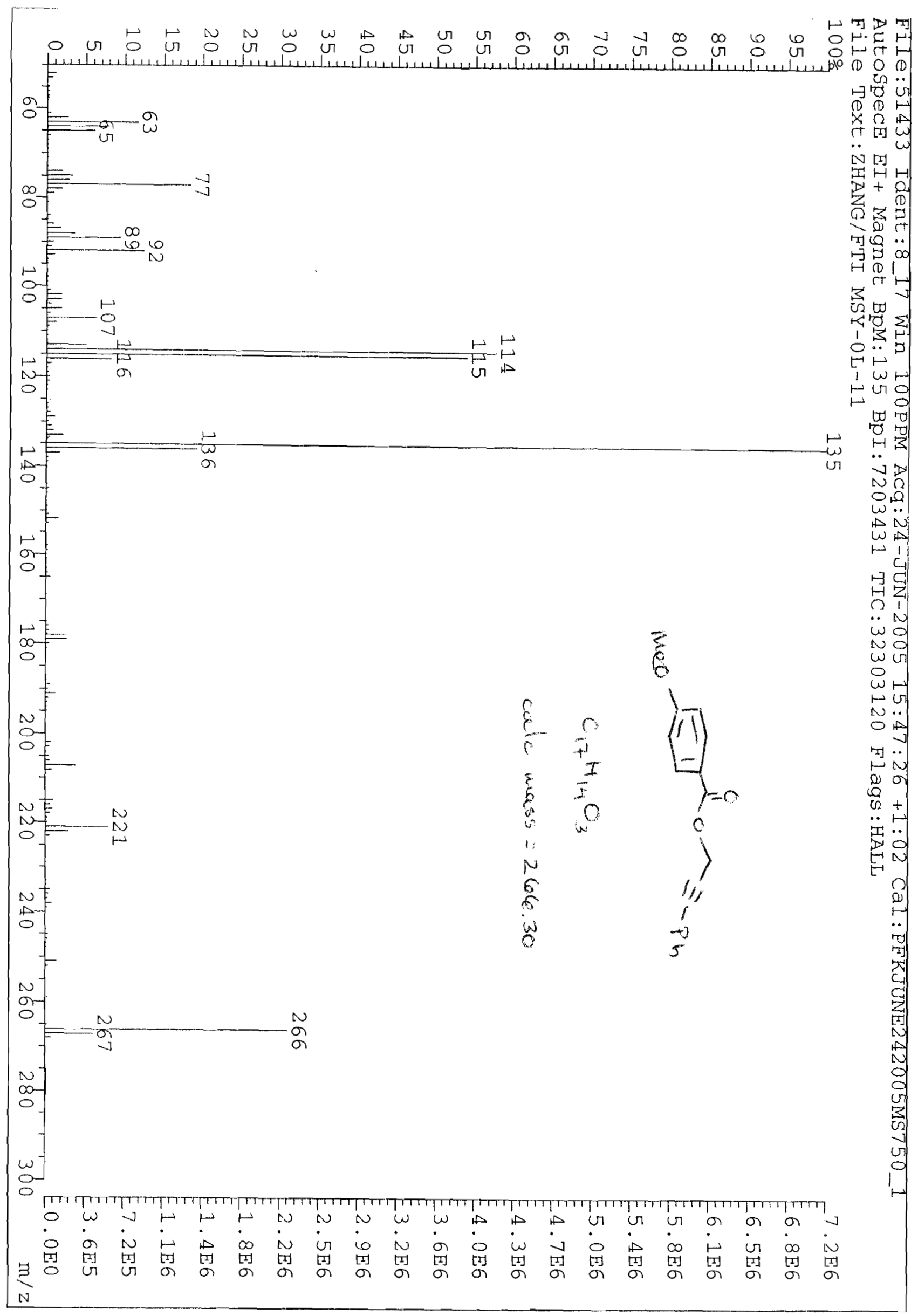


- SEO GTFET

FUH \# 493 HFF 8,2094 95:33:15

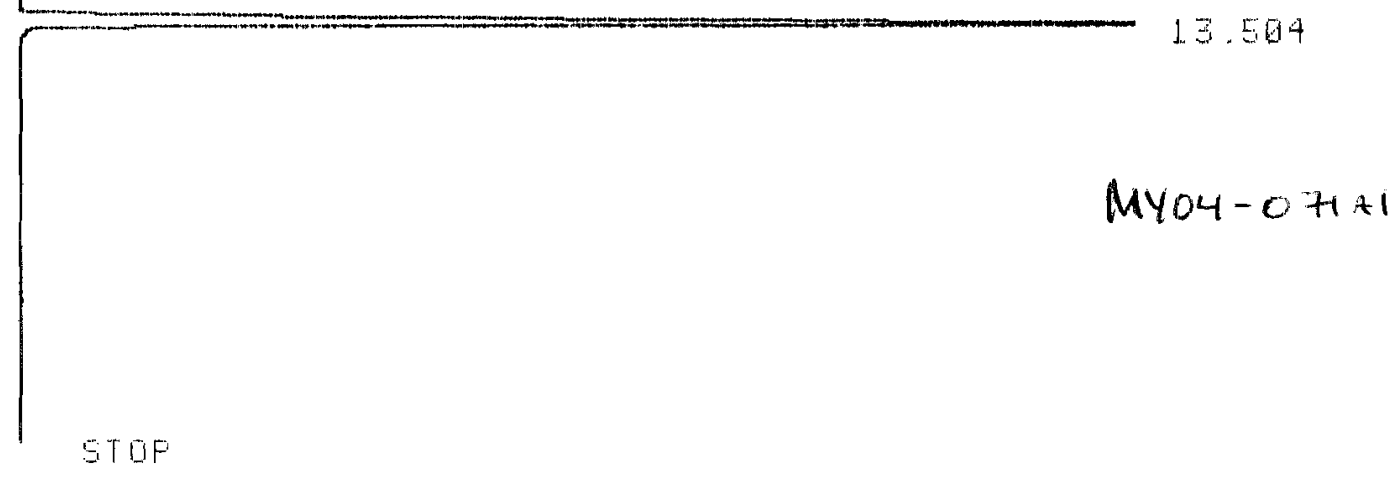

FUNH 40

FFP $\quad 9,2004 \quad 05: 53: 15$

SFMFLE\# 1

HREAX

\begin{tabular}{|c|c|c|c|c|}
\hline RT & AEEF & TYFE & WIDTH & AREF \\
\hline 6.855 & $E \in 5$ & $P E$ & .036 & .30735 \\
\hline 6.945 & 3000 & $\mathrm{EE}$ & .946 & 1.38553 \\
\hline 180 & 1743 & $\mathrm{~EB}$ & 690 & 80558 \\
\hline 584 & 21095 & $\mathrm{FE}$ & 144 & 97. \\
\hline
\end{tabular}

TOTAL AREA = 21636?

MUL FACTOE=1. GQGEE+ GE

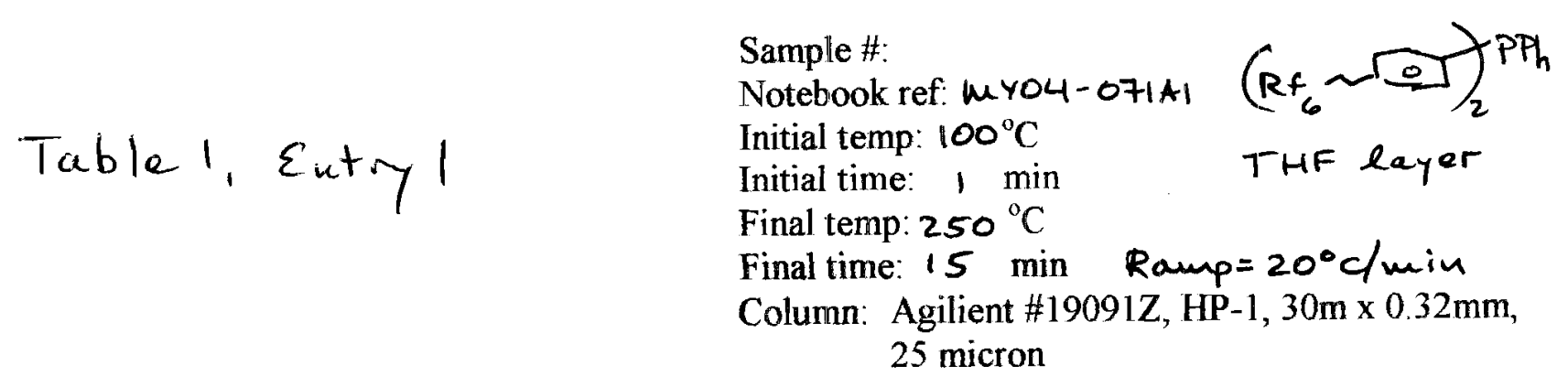




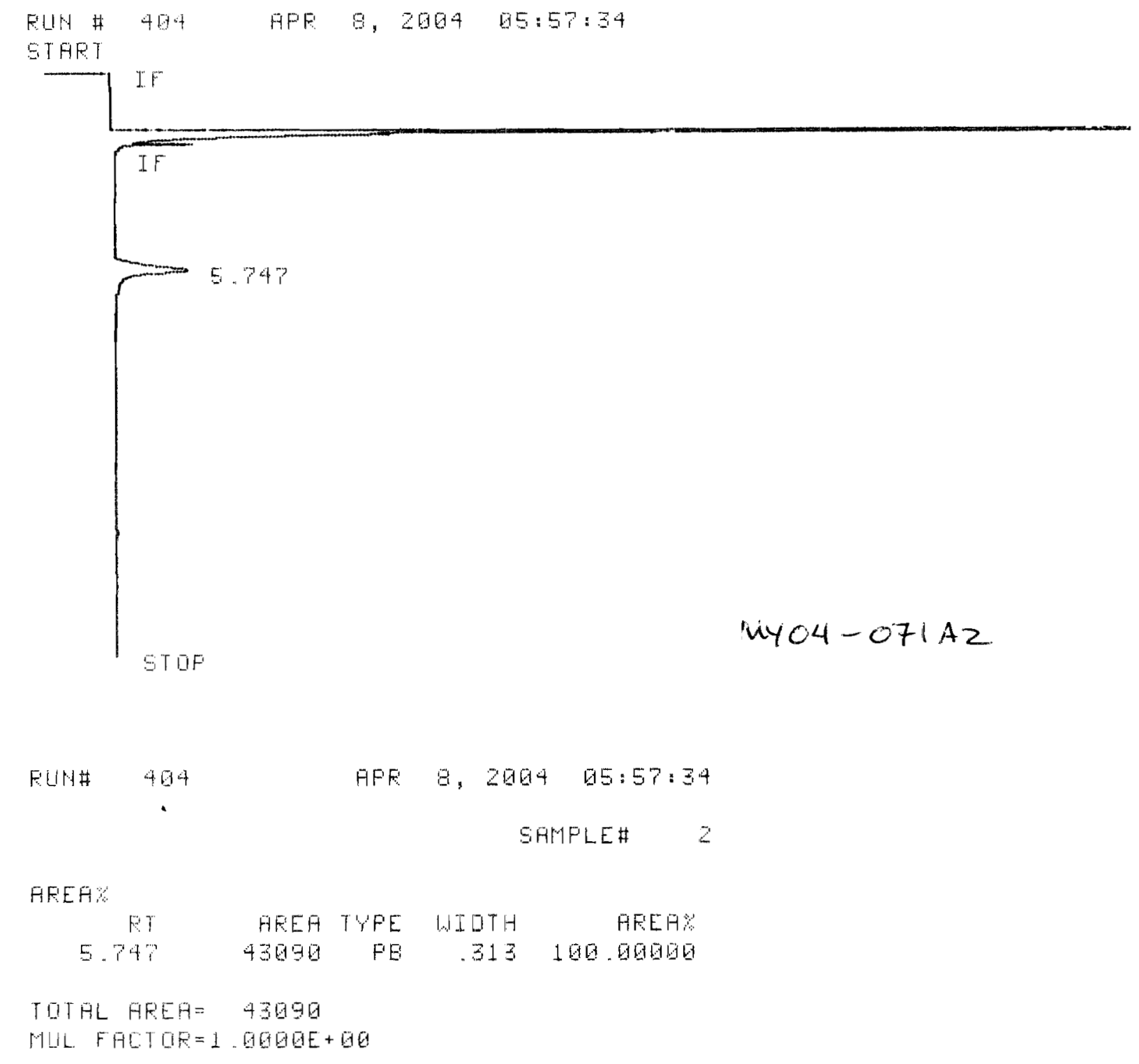

Tuble 1, Entry 1

Sample \#:

Notebook ref: Myo4-071A2 Initial temp: $100^{\circ} \mathrm{C}$ Initial time: $1 \mathrm{~min}$ Final temp: $250^{\circ} \mathrm{C}$ $\left(R f_{6} \sqrt{0}\right)_{2}^{P P h}$ $\mathrm{FC}-72$ layer

Final time: $15 \mathrm{~min} R_{a m p}=20^{\circ} \mathrm{C} / \mathrm{min}$ Column: Agilient \#19091Z, HP-1, 30m x 0.32mm, 25 micron 


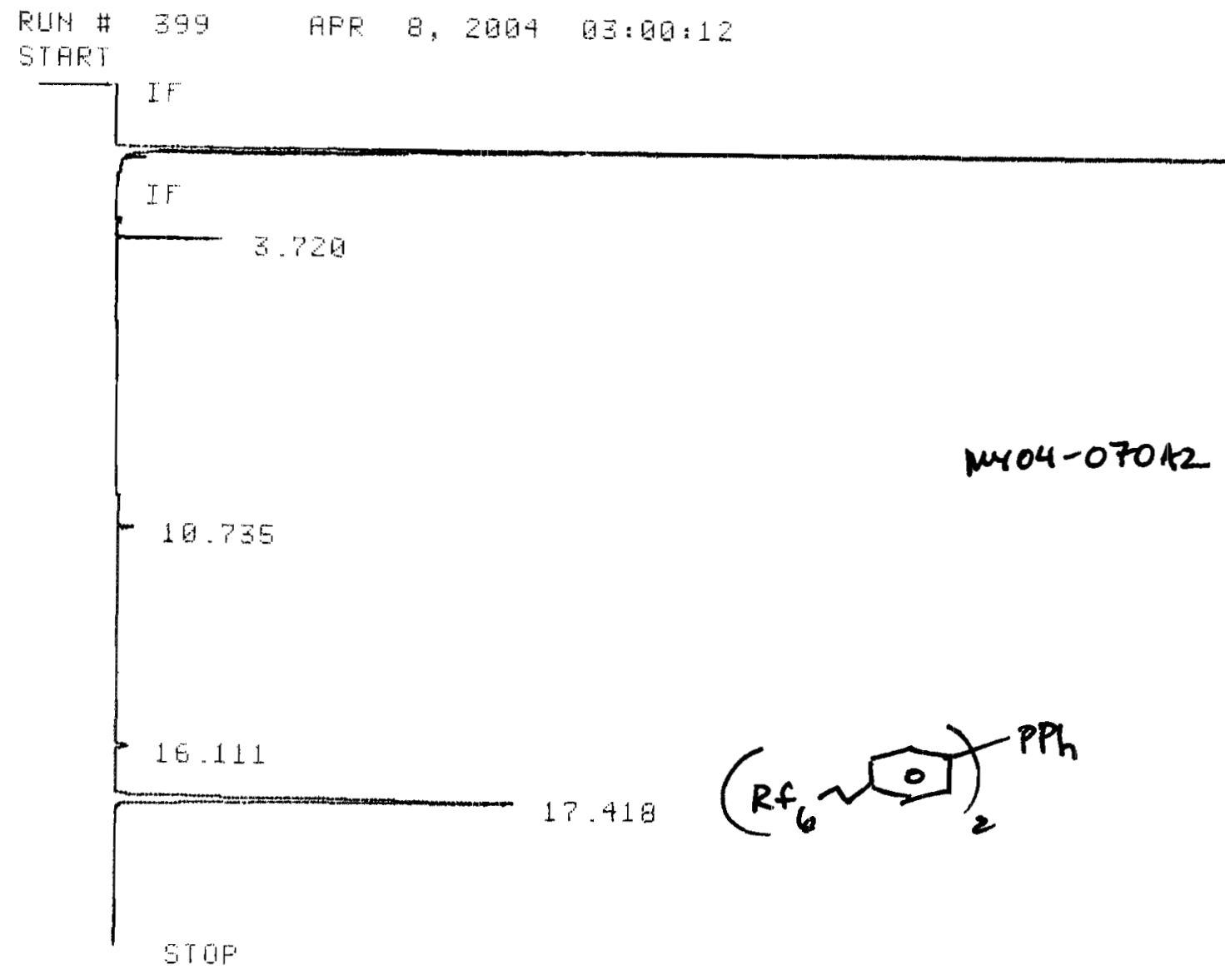

FutH 395

AFR $\quad$ 9, 2004 03: 90:12

SAMPLE\#

HFE

\begin{tabular}{|c|c|c|c|c|}
\hline ET & AREA & TYPE & $M I D H$ & AFEEA \\
\hline $3.72 \mathrm{a}$ & 3365 & EB & .020 & 4.28564 \\
\hline 19.735 & 1286 & UB & .947 & 1. 63784 \\
\hline $1 E .111$ & 2048 & $\mathrm{~EB}$ & .095 & 2.80832 \\
\hline 17.419 & $7 \pm 819$ & $\mathrm{Eg}$ & .114 & $91 .+60$ \\
\hline
\end{tabular}

TOTAL AFEA = 7OSLB

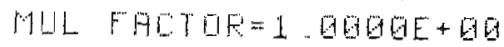

Table 1, Eutry 2

Sample \#:
Notebook ref: WY04-070B 1 (Rf 107$)_{2}^{\circ P h}$
Initial temp: $50^{\circ} \mathrm{C}$

Initial time: $1 \mathrm{~min} \quad \mathrm{CH}_{3} \mathrm{CN}$ layer

Final time: $10 \mathrm{~min}$ Ramp $=20^{\circ} \mathrm{C} /$ min

Column: Agilient \#19091Z, HP-1,30m x 0.32mm, 25 micron 


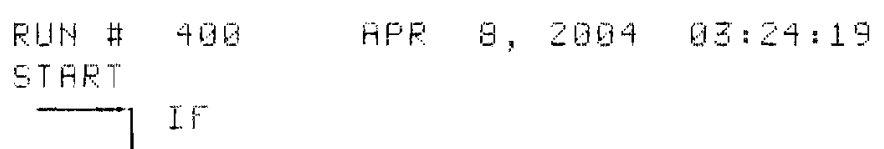

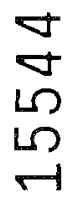
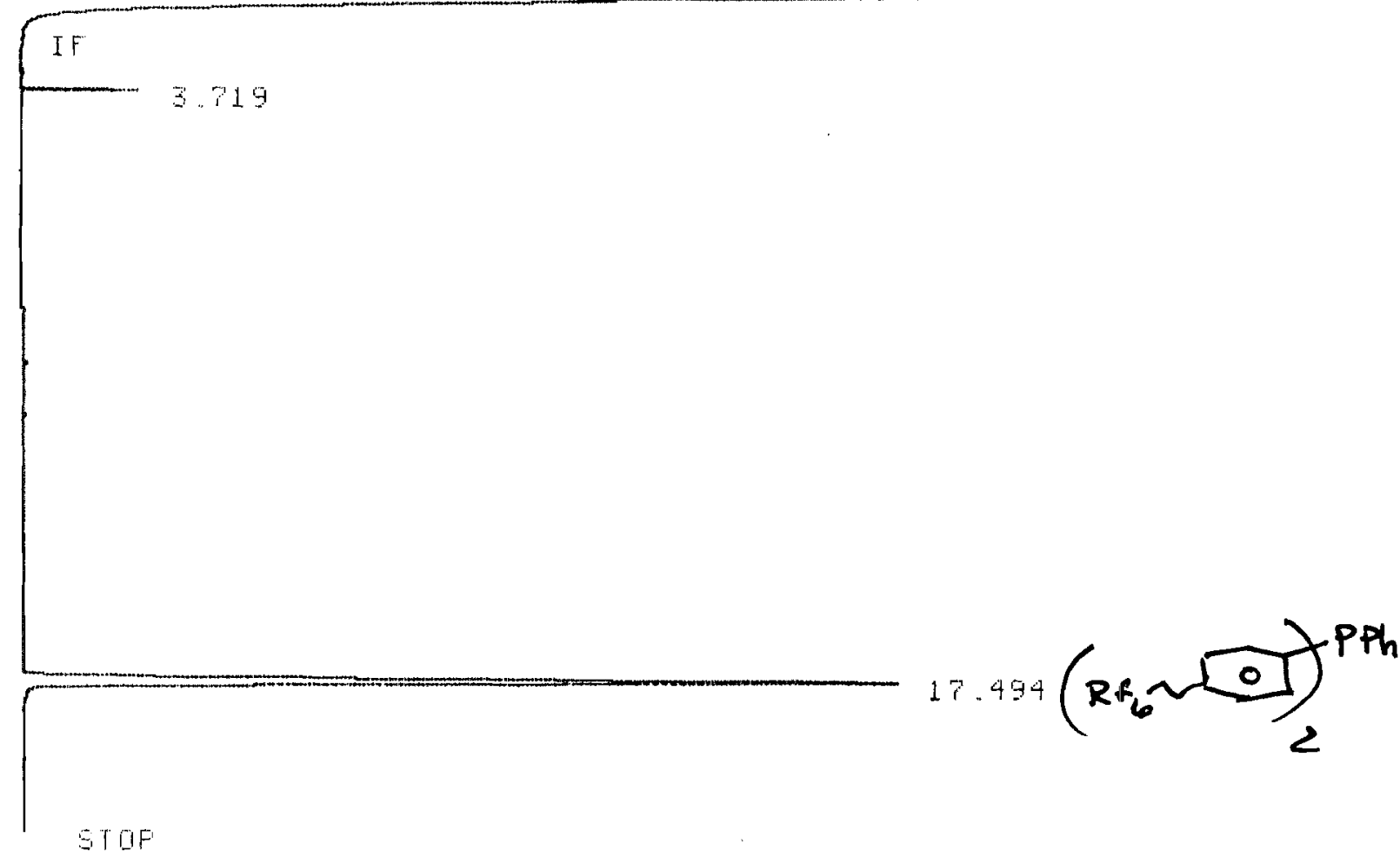

MY04-070B2

FuN 400

HFF $\quad 9,2694 \quad 03: 24: 19$

EHMPLE

4

HEEH.

$\begin{array}{rrrrr}\text { FT } & \text { AFEF TYPE } & \text { WIOTH } & \text { AFEH } \\ 3.719 & 3635 & \text { EE } & .029 & 2.09642 \\ 17.494 & 179977 & \text { EE } & .135 & 97.99958\end{array}$

TOTAL HEEA= 191212

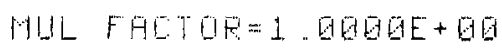

Table i, Eutry 2

Sample \#: Initial temp: $50^{\circ} \mathrm{C}$

FC- 72 layer

Initial time: I min

Final temp: $250^{\circ} \mathrm{C}$

Final time: $10 \mathrm{~min}$ Ramp $=20^{\circ} \mathrm{C} / \mathrm{min}$

Column: Agilient \#19091Z, HP-1, 30m x 0.32mm, 25 micron

Page 36 of 126 


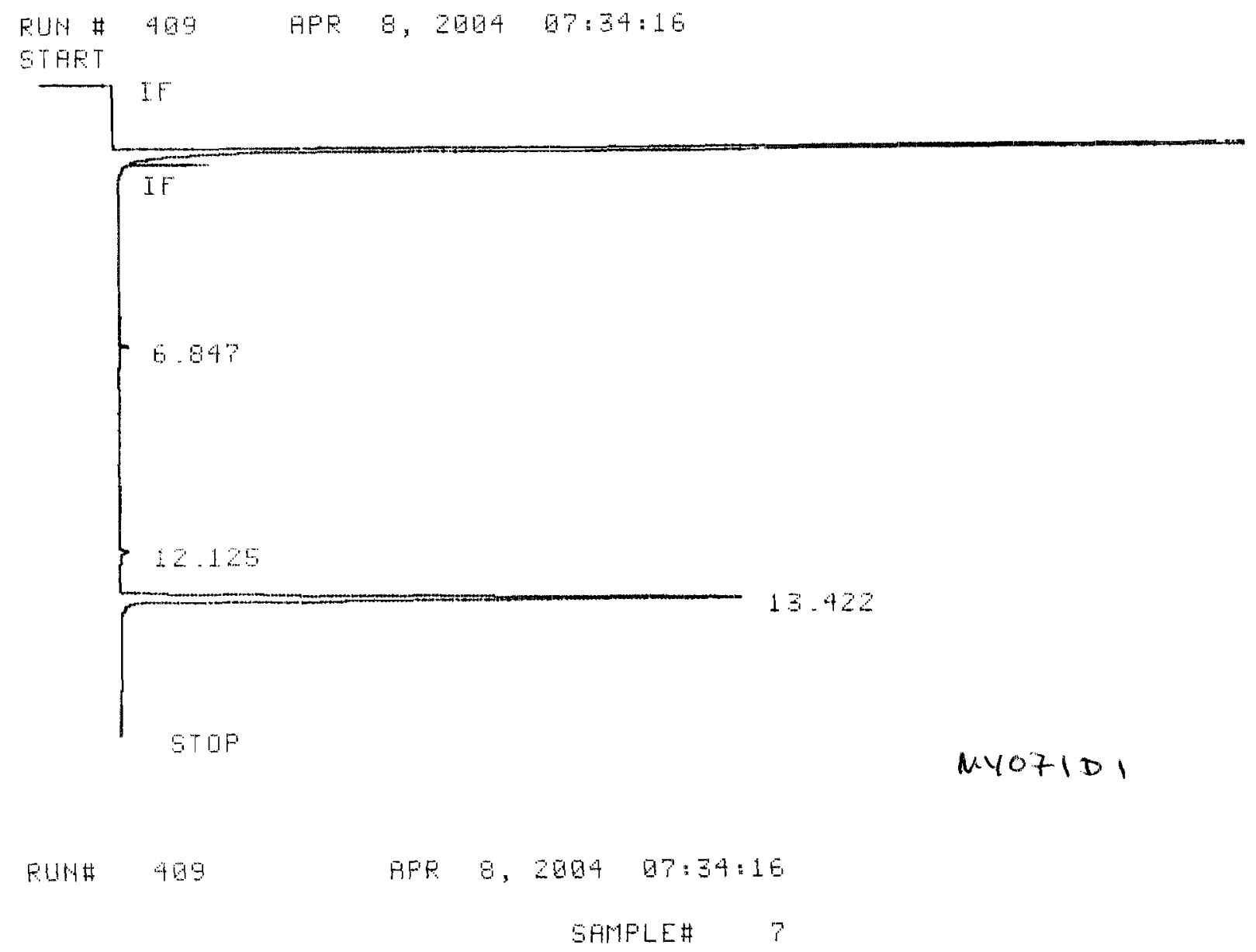

AFEHY

\begin{tabular}{|c|c|c|c|c|}
\hline$F r$ & AFEH & TYPE & $\omega I[1 T H$ & AFEAK \\
\hline 6.847 & $19 E 2$ & FE & .051 & .79841 \\
\hline 2.125 & 1656 & $11 \mathrm{E}$ & 102 & 1.23250 \\
\hline 42 & 131648 & $P E$ & $12 \theta$ & 977 \\
\hline
\end{tabular}

TOTAL AEEA = 134351

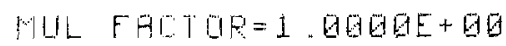

Table 1, Entry 3

Sample \#:

Notebook ref: MyO4-07101 Initial temp: $100^{\circ} \mathrm{C}$ Initial time: $1 \mathrm{~min}$ Final temp: $250{ }^{\circ} \mathrm{C}$

Final time: $15 \mathrm{~min}$ Ramp $=20^{\circ} \mathrm{C} / \mathrm{min}$

Column: Agilient \#19091Z, HP-1, 30m x 0.32mm, 25 micron 


RUH \# 410 AFF 8,2084 G7:53:34
STAFT
$\longrightarrow$ IF

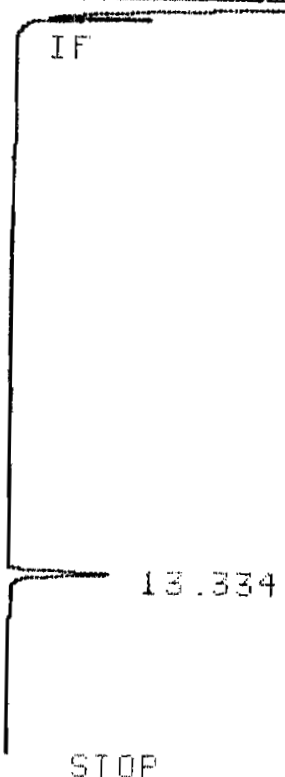

$D 2$

RUAH 410

HFE $\quad 8,2004$ 07:53:34

SAMFLE\# 8

AFEFE?
$13 \quad 334$
FEEA TYFE WIUTH
16419 EE .125
AREA\%
100.06090

TUTAL AREA = 16418

MUL FHET IF $=1$. $8000 E+00$

Table 1, Eutry 3

Sample \#:

Notebook ref: $M$ Yo4-071D2 Initial ternp: $100^{\circ} \mathrm{C}$ Initial time: $1 \mathrm{~min}$ Final temp: $250^{\circ} \mathrm{C}$

Final time: $15 \mathrm{~min}$ Ramp $=20^{\circ} \mathrm{C} / \mathrm{m}$ in

Column: Agilient \#19091Z, HP-1, 30m x 0.32mm, 25 micron 


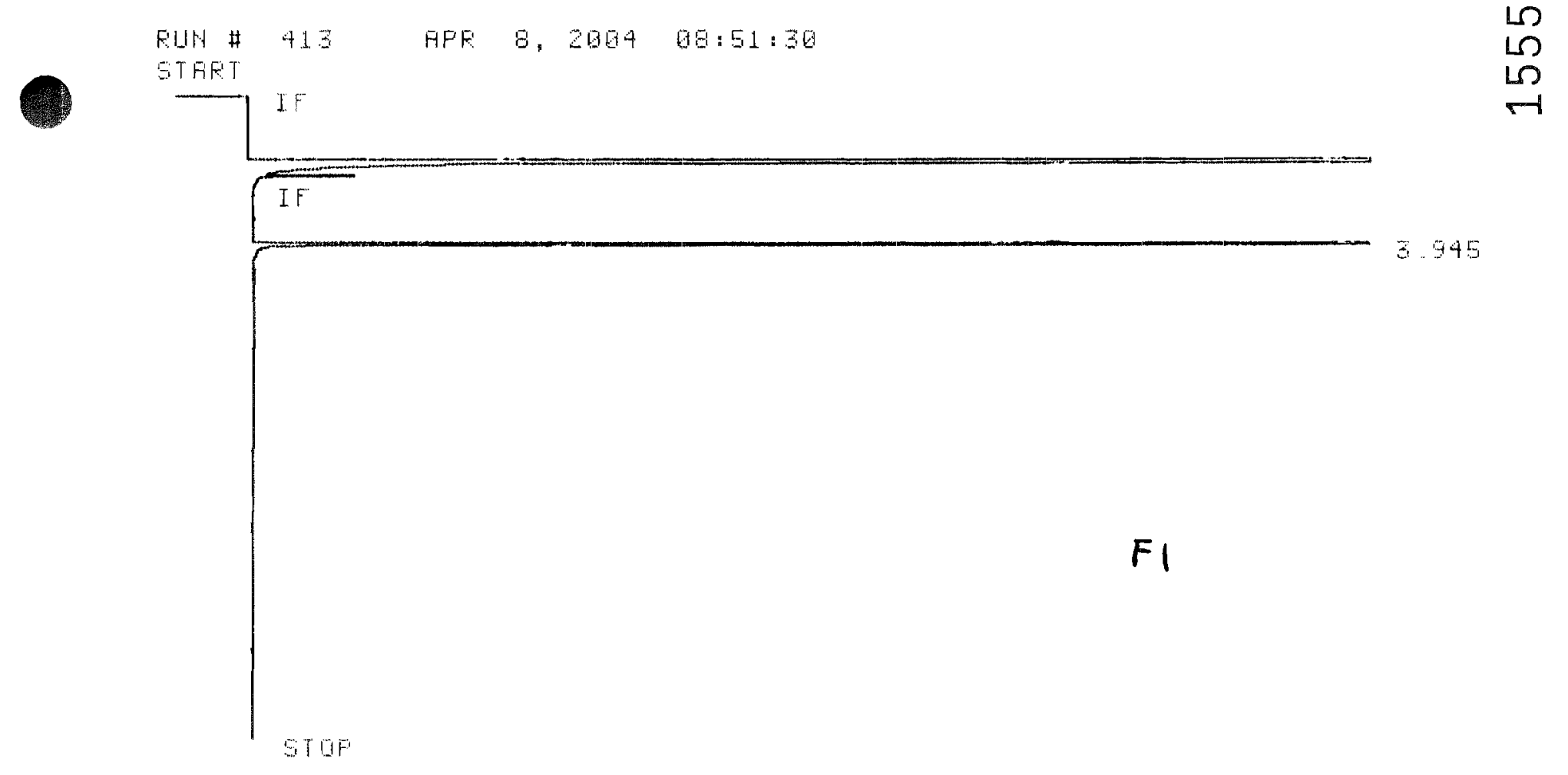

FulH 413

AFE $8,2004 \quad 09: 51: 30$

SAMFLEH 11

AREAR
3.945
AFEA TYPE WIDTH
AREF:
3.945
$370957 \quad P E$
.032
100.09006

TOTAL AFEA = 37G957

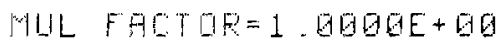

Table 1, Eutry 4

Sample \#: $\mathrm{PPH}_{3}$ DMF layar

Notebook ref: MyO 4-07IFI

Initial temp: $100^{\circ} \mathrm{C}$

Initial time: $1 \mathrm{~min}$

Final temp: $250^{\circ} \mathrm{C}$

Final time: 15 min $R$ amp $=20^{\circ} \mathrm{C}$ wain

Column: Agilient \#19091Z, HP-1,30m x 0.32mm, 25 micron 


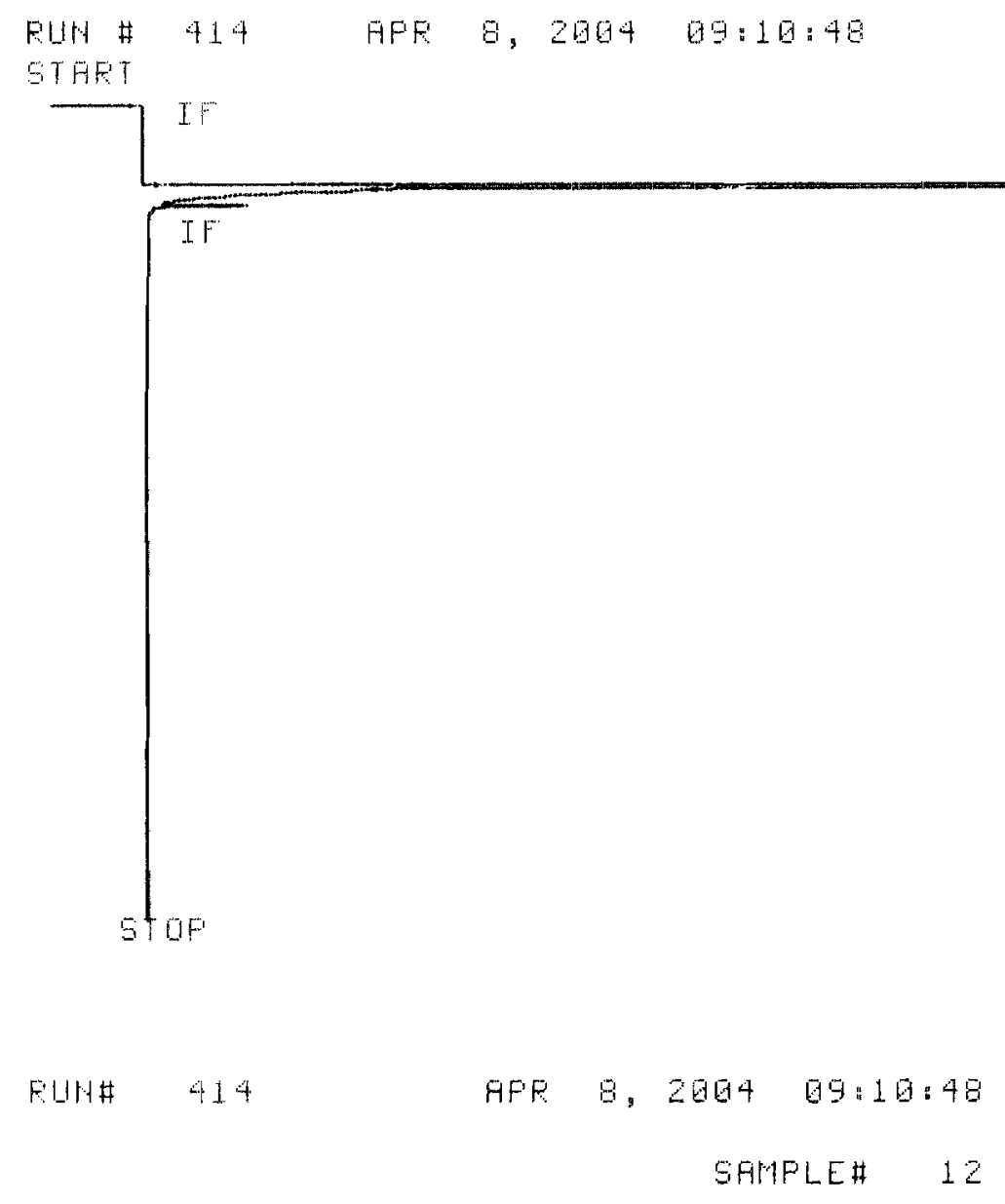

$F_{2}$

WO PUN FEFKS GTOFED

Table 1, Entry 4

Sample \#: $\mathrm{PPh}_{3} \quad \mathrm{FC}-7 \mathrm{C}$ layer

Notebook ref: $M Y 04-0 Z \backslash F 2$

Initial temp: $100{ }^{\circ} \mathrm{C}$

Initial time: $1 \mathrm{~min}$

Final temp: $250^{\circ} \mathrm{C}$

Final time: $15 \mathrm{~min} R_{\text {aump }}=20^{\circ} \mathrm{C} / \mathrm{min}$

Column: Agilient \#19091Z, HP-1, 30m x 0.32mm, 25 micron 


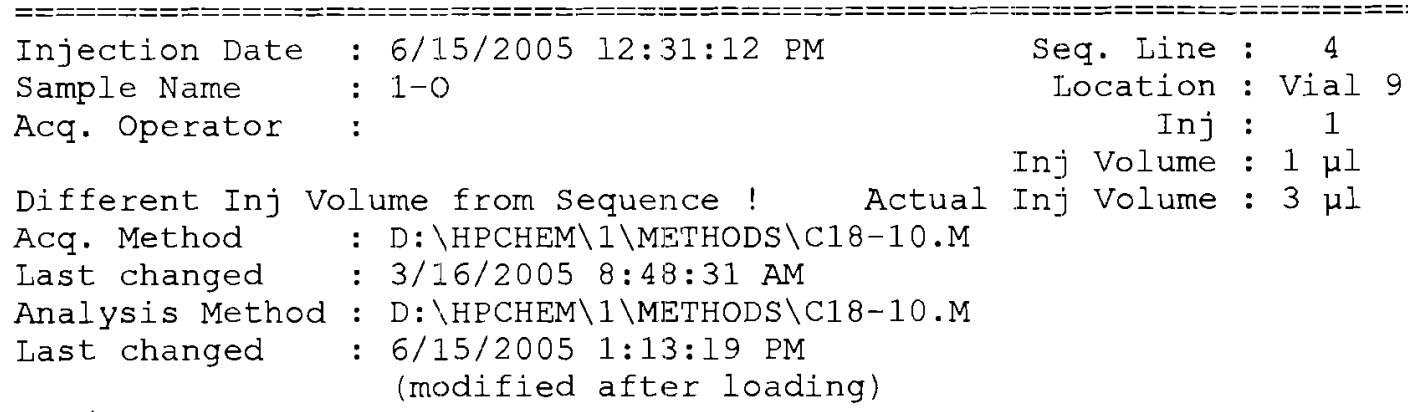

$\mathrm{C} 18 / \mathrm{MeCN}-\mathrm{H} 2 \mathrm{O}-\mathrm{TFA}$

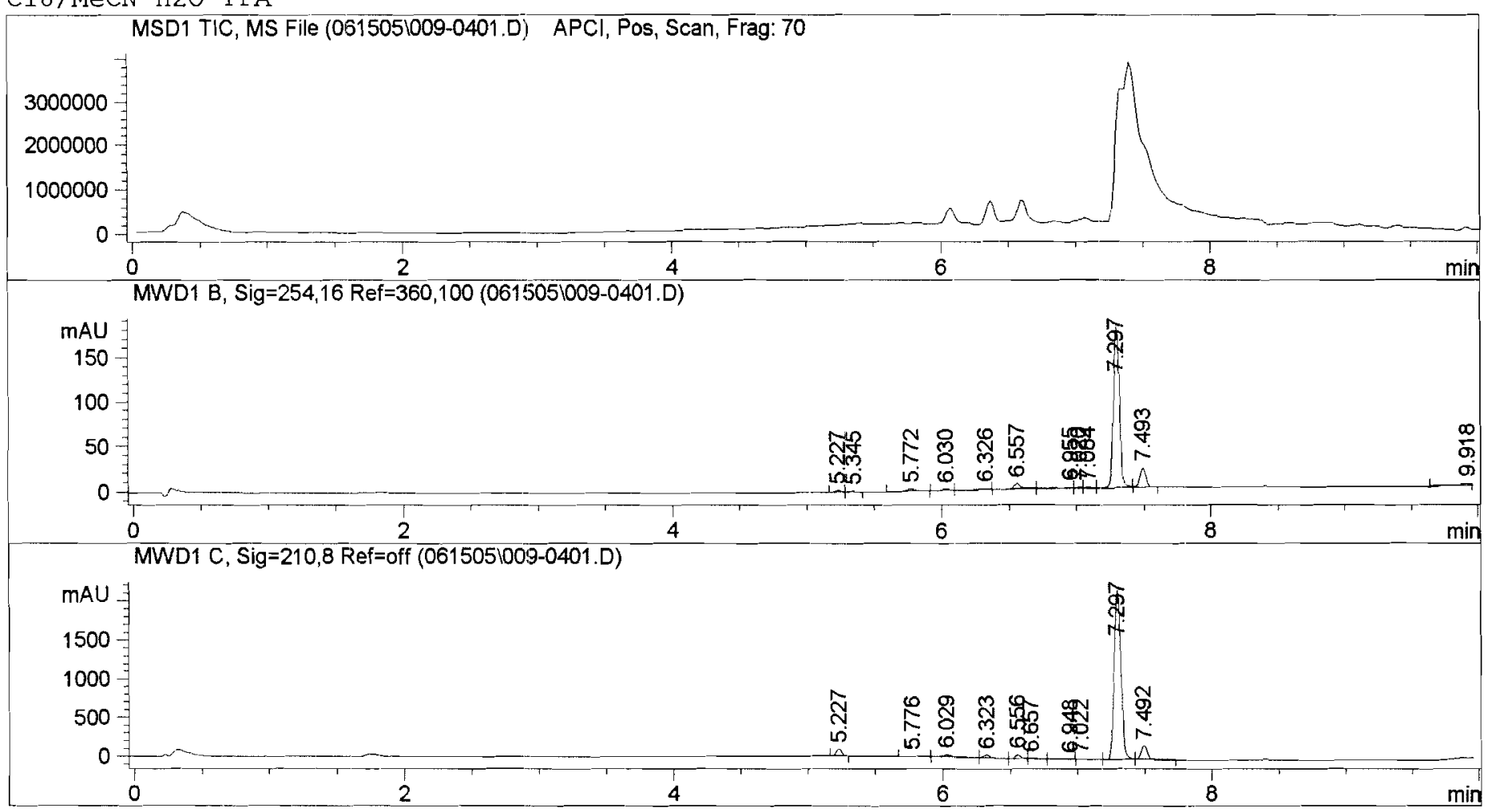

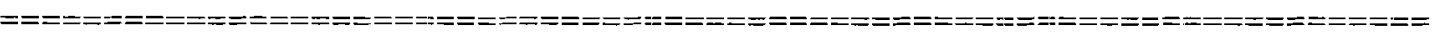

Area Percent Report

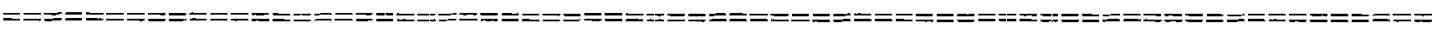

Sorted BY

Multiplier

Dilution
Signal

1.0000

1.0000

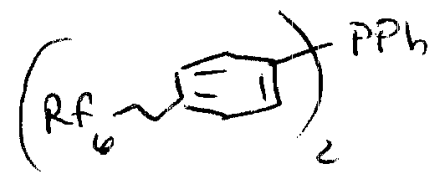

Signal 1: MSD1 TIC, MS File

Signal 2: MWD1 B, Sig $=254,16$ Ref $=360,100$

$\mathrm{FC}-72 / 5 \% \mathrm{H}_{2} \mathrm{O}$ inTHF

\begin{tabular}{|c|c|c|c|c|c|c|}
\hline $\begin{array}{c}\text { Peak } \\
\#\end{array}$ & $\begin{array}{c}\text { RetTime } \\
\text { [min] }\end{array}$ & Type & $\begin{array}{l}\text { Width } \\
\text { [min] }\end{array}$ & $\begin{array}{c}\text { Area } \\
{\left[\mathrm{mAU}^{*} \mathrm{~s}\right]}\end{array}$ & $\begin{array}{l}\text { Height } \\
\text { [mAU] }\end{array}$ & $\begin{array}{c}\text { Area } \\
\frac{8}{8}\end{array}$ \\
\hline 1 & 5.227 & PV & 0.0442 & 6.08072 & 2.20549 & 0.7994 \\
\hline 2 & 5.345 & $\mathrm{VP}$ & 0.0491 & 3.24422 & 1.07844 & 0.4265 \\
\hline 3 & 5.772 & PV & 0.0817 & 10.66230 & 2.01744 & 1. 4018 \\
\hline 4 & 6.030 & VV & 0.0550 & 6.58384 & 1.87351 & 0.8656 \\
\hline 5 & 6.326 & VV & 0.0719 & 8.80093 & 1.71510 & 1.1571 \\
\hline 6 & 6.557 & $\mathrm{VV}$ & 0.0723 & 33.57156 & 6.49933 & 4.4137 \\
\hline 7 & 6.955 & VV & 0.1646 & 19.33217 & 1.51776 & 2.5416 \\
\hline 8 & 7.020 & VV & 0.0487 & 6.17910 & 1.68905 & 0.8124 \\
\hline 9 & 7.084 & VV & 0.0680 & 7.83137 & 1.63060 & 1.0296 \\
\hline
\end{tabular}

orgonic lagor

Tuble 1, Entry 5 
Data File D: \HPCHEM $\backslash 1 \backslash D A T A \backslash 061505 \backslash 009-0401 . D$

\begin{tabular}{|c|c|c|c|c|c|c|}
\hline $\begin{array}{c}\text { Peak } \\
\#\end{array}$ & $\begin{array}{c}\text { RetTime } \\
\text { [min] }\end{array}$ & Type & $\begin{array}{l}\text { Width } \\
\text { [rin] }\end{array}$ & $\begin{array}{c}\text { Area } \\
{\left[\mathrm{mAU}^{\star} \mathrm{s}\right]}\end{array}$ & $\begin{array}{l}\text { Height } \\
\text { [mAU] }\end{array}$ & $\begin{array}{c}\text { Area } \\
\frac{8}{8}\end{array}$ \\
\hline 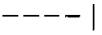 & ------ & & & --------- & --------- & \\
\hline 10 & 7.297 & VV & 0.0505 & 562.34534 & 179.72046 & 73.9323 \\
\hline 11 & 7.493 & VB & 0.0503 & 70.49876 & 21.48973 & 9.2686 \\
\hline 12 & 9.918 & $B B A$ & 0.1500 & 25.49146 & 2.83288 & 3.3514 \\
\hline Total & : & & & 760.62178 & 224.26979 & \\
\hline
\end{tabular}

Signal 3: MWD1 C, Sig=210,8 Ref=off

\begin{tabular}{|c|c|c|c|c|c|c|}
\hline $\begin{array}{c}\text { Peak } \\
\#\end{array}$ & $\begin{array}{c}\text { RetTime } \\
\text { [min] }\end{array}$ & Type & $\begin{array}{l}\text { Width } \\
\text { [rnin] }\end{array}$ & $\begin{array}{c}\text { Area } \\
{\left[\mathrm{mAU}^{*} \mathrm{~s}\right]}\end{array}$ & $\begin{array}{l}\text { Height } \\
\text { [mAU] }\end{array}$ & $\begin{array}{c}\text { Area } \\
\quad 8\end{array}$ \\
\hline 1 & 5.227 & $\mathrm{BB}$ & 0.0449 & 252.38884 & 89.38633 & 2.7591 \\
\hline 2 & 5.776 & BV & 0.1147 & 81.41609 & 9.61943 & 0.8900 \\
\hline 3 & 6.029 & VV & 0.0911 & 215.12369 & 32.56408 & 2.3517 \\
\hline 4 & 6.323 & VV & 0.0584 & 144.33784 & 37.89673 & 1.5779 \\
\hline 5 & 6.556 & VV & 0.0475 & 159.92114 & 52.58974 & 1.7482 \\
\hline 6 & 6.657 & VP & 0.0420 & 14.70882 & 5.72650 & 0.1608 \\
\hline 7 & 6.948 & VV & 0.0379 & 6.68689 & 3.01637 & 0.0731 \\
\hline 8 & 7.022 & VP & 0.0634 & 26.34241 & 5.75634 & 0.2880 \\
\hline 9 & 7.297 & VV & 0.0571 & 7668.80713 & 2177.50684 & 83.8342 \\
\hline 10 & 7.492 & VB & 0.0489 & 577.85687 & 182.65897 & 6.3170 \\
\hline
\end{tabular}

Totals :

$9147.58973 \quad 2596.72132$

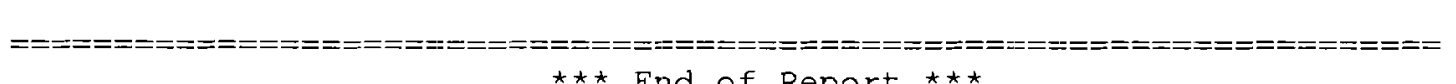




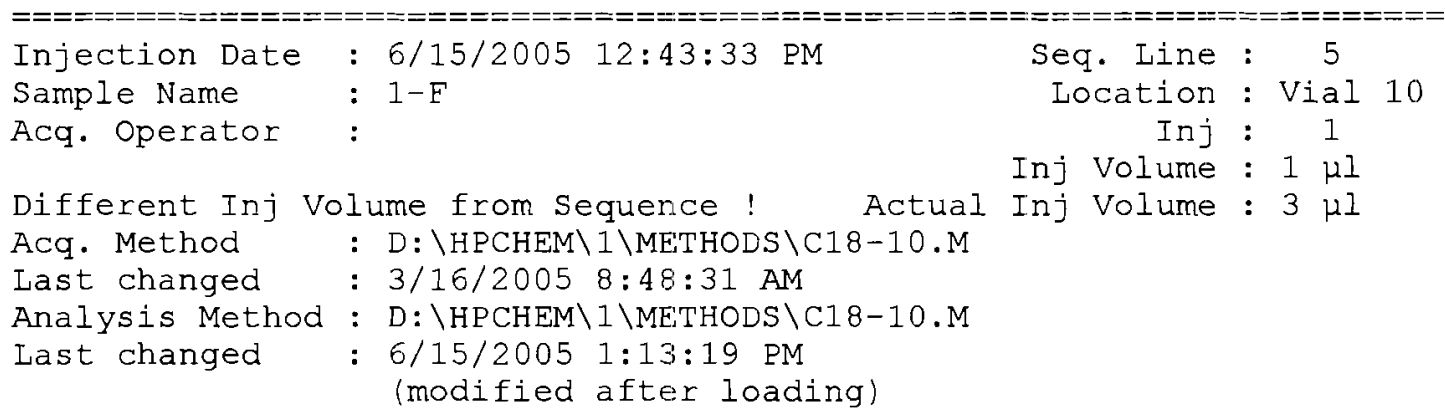

$\mathrm{C} 18 / \mathrm{MeCN}-\mathrm{H} 20-\mathrm{TFA}$

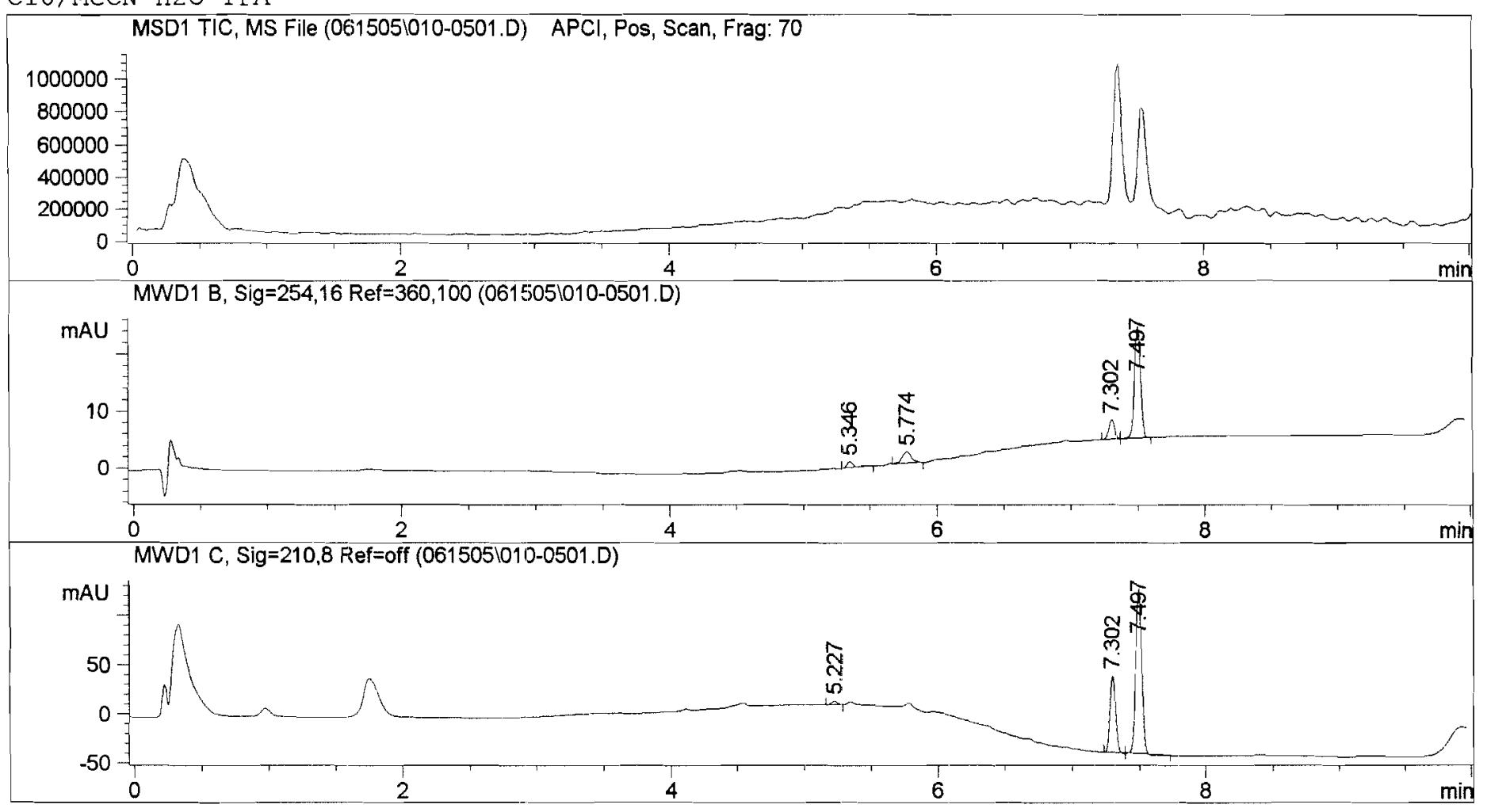

Area Percent Report

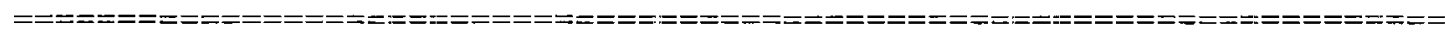

$\begin{array}{lll}\text { Sorted By } & : & \text { Signal } \\ \text { Multiplier } & : & 1.0000 \\ \text { Dilution } & : & 1.0000\end{array}$

Signal 1: MSDl TIC, MS File

Signal 2: MWD1 B, Sig=254,16 Ref=360,100

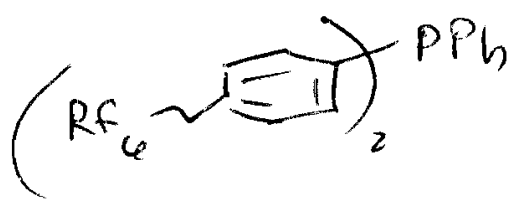

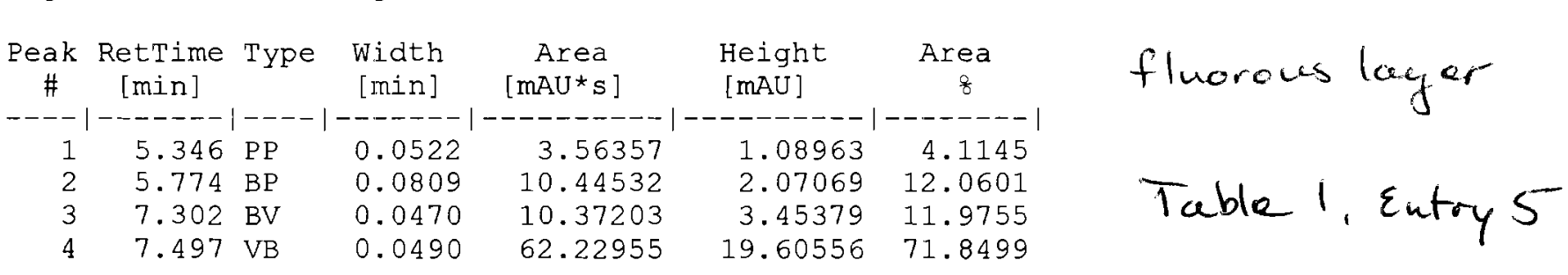

Totals :

$86.61048 \quad 26.21967$ 
Signal 3: MWD1 C, Sig=210,8 Ref:off

\begin{tabular}{|c|c|c|c|c|c|c|}
\hline $\begin{array}{c}\text { Peak } \\
\#\end{array}$ & $\begin{array}{l}\text { RetTime } \\
\text { [min] }\end{array}$ & Type & $\begin{array}{l}\text { Width } \\
\text { [nin] }\end{array}$ & $\begin{array}{c}\text { Area } \\
{\left[\mathrm{MAU}^{\star} \mathrm{S}\right]}\end{array}$ & $\begin{array}{l}\text { Height } \\
\text { [mA.U] }\end{array}$ & $\begin{array}{c}\text { Area } \\
\frac{q}{q}\end{array}$ \\
\hline 1 & 5.227 & BB & 0.0512 & 12.86199 & 3.82534 & 1.6784 \\
\hline 2 & 7.302 & $\mathrm{BP}$ & 0.0461 & 230.31741 & 78.72060 & 30.0556 \\
\hline 3 & 7.497 & VB & 0.0482 & 523.12396 & 168.70514 & 68.2659 \\
\hline
\end{tabular}

Totals :

$766.30336 \quad 251.25108$ 

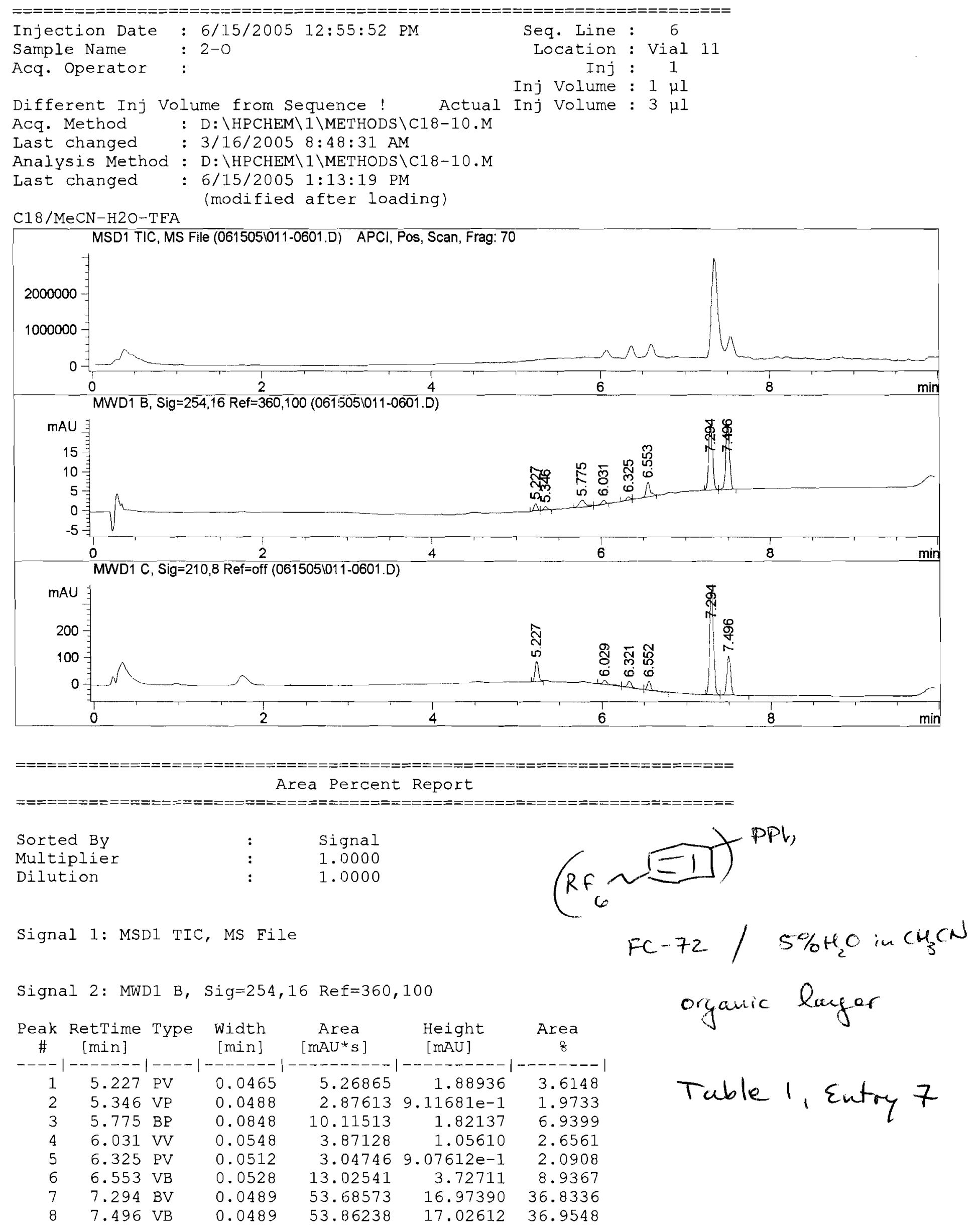

Totals :
$145.75218 \quad 44.31325$

Signal 3: MWD1 C, Sig=210,8 Ref=off

\begin{tabular}{|c|c|c|c|c|c|c|}
\hline $\begin{array}{c}\text { Peak } \\
\#\end{array}$ & $\begin{array}{c}\text { RetTime } \\
\text { [min] }\end{array}$ & Type & $\begin{array}{l}\text { Width } \\
\text { [min] }\end{array}$ & $\begin{array}{c}\text { Area } \\
{\left[m A U^{\star} s\right]}\end{array}$ & $\begin{array}{l}\text { Height } \\
\text { [mAU] }\end{array}$ & $\begin{array}{c}\text { Area } \\
\frac{g}{8}\end{array}$ \\
\hline 1 & 5.227 & $B B$ & 0.0467 & 22607861 & $77 \quad 44226$ & 10.5580 \\
\hline 2 & 6.029 & BV & 0.0704 & 81.91152 & 16.35591 & 3.8253 \\
\hline 3 & 6.321 & VV & 0.0507 & 78.94899 & 23.79880 & 3.6870 \\
\hline 4 & 6.552 & VB & 0.0412 & 75.39247 & 32.29431 & 3.5209 \\
\hline 5 & 7.294 & BV & 0.0485 & 1227.50645 & 392.74875 & 57.3298 \\
\hline 6 & 7.496 & VB & 0.0479 & 451.36880 & 146.53583 & 21.0791 \\
\hline Total & : & & & 2141.30685 & 689.17586 & \\
\hline
\end{tabular}

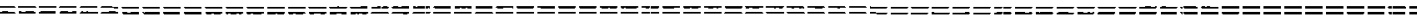



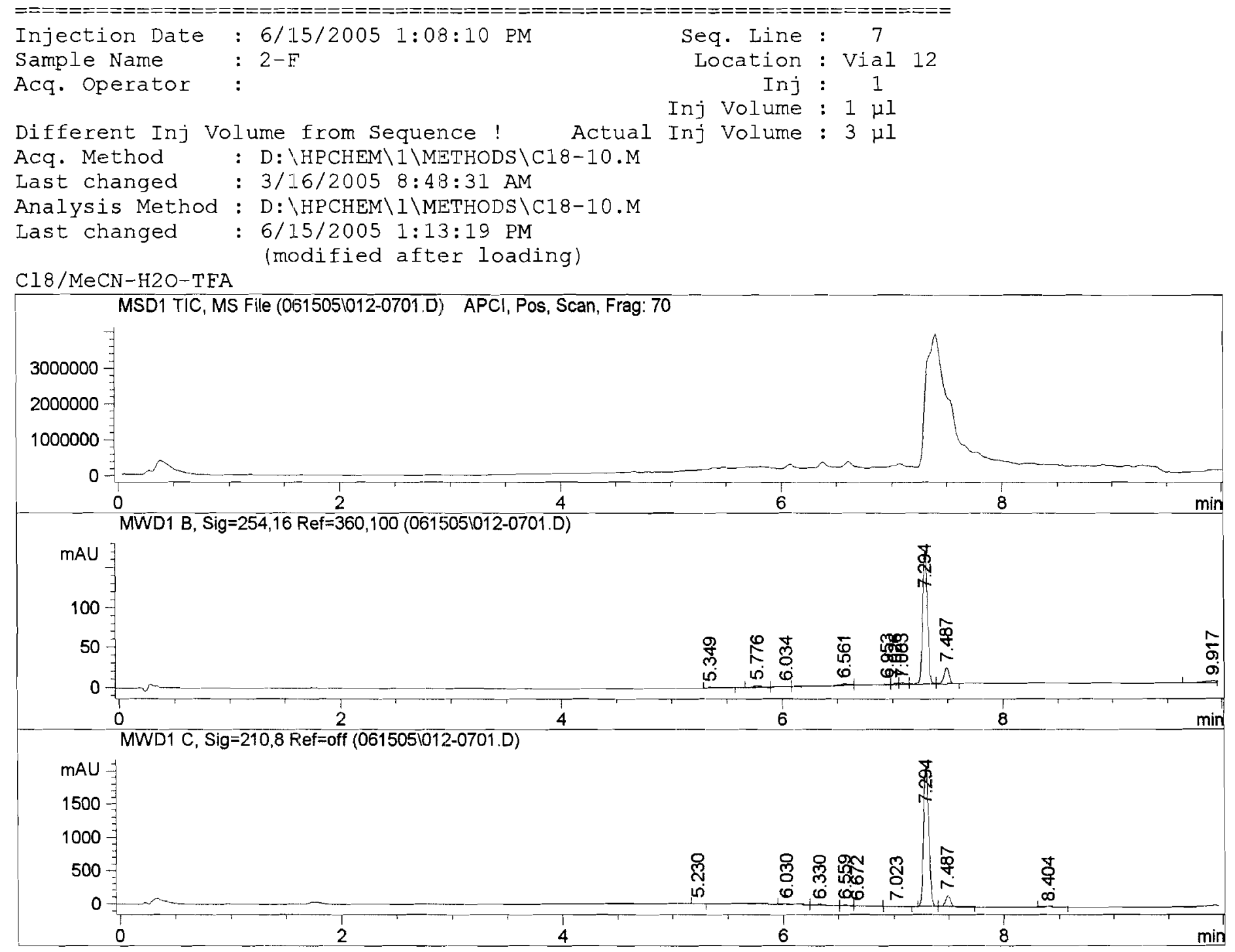

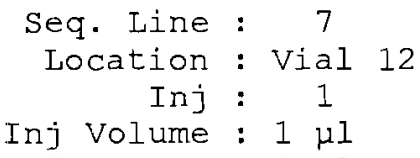

Area Percent Report

$\begin{array}{lll}=== & ==0========== & ===== \\ \text { Sorted By } & : & \text { Signal } \\ \text { Multiplier } & : & 1.0000 \\ \text { Dilution } & : & 1.0000\end{array}$

Signal 1: MSD1 TIC, MS File

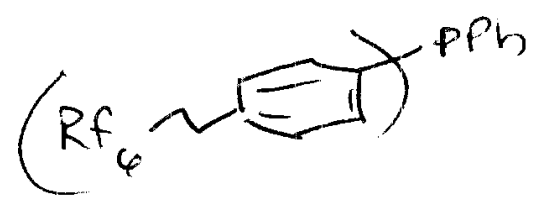

$$
\mathrm{FC}-72 / 5 \% \mathrm{H}_{2} \mathrm{O} \text { in } \mathrm{CH}_{3} \mathrm{CN}
$$

Signal 2: MWD1 B, Sig=254,16 Ref $=360,100$

\begin{tabular}{|c|c|c|c|c|c|c|}
\hline ?eak & $\begin{array}{c}\text { RetTime } \\
\text { [min] }\end{array}$ & Type & $\begin{array}{l}\text { Width } \\
{[\min ]}\end{array}$ & $\begin{array}{c}\text { Area } \\
{\left[\mathrm{mAU}^{\star} \mathrm{s}\right]}\end{array}$ & $\begin{array}{l}\text { Height } \\
{[\mathrm{mAU}]}\end{array}$ & $\begin{array}{c}\text { Area } \\
\frac{8}{8}\end{array}$ \\
\hline 1 & 5.349 & $\mathrm{PP}$ & 0.0510 & 3.49789 & 1.10148 & 0.5138 \\
\hline 2 & 776 & $\mathrm{BP}$ & 0.0821 & 10.68223 & 2.07375 & 1.5691 \\
\hline 3 & 6.034 & VV & 0.0714 & 4.16317 & $8.17336 e-1$ & 0.6115 \\
\hline 4 & 6.561 & VV & 0.1 & 28.0 & 2.542 & 4.1247 \\
\hline 5 & 6.953 & VV & 0.1829 & 22.43927 & 1.53229 & 3.2960 \\
\hline 6 & 7.026 & VV & 0.0563 & 6.35988 & 1.67358 & 0.9342 \\
\hline 7 & 7.083 & VV & 0.0 & 7.1 & 1.5 & 1.0472 \\
\hline 8 & 7.294 & VV & 0.0492 & 503.90280 & 167.24901 & 74.0166 \\
\hline 9 & 7.487 & VB & 0.0538 & 68.25624 & 20.00123 & 10.0259 \\
\hline
\end{tabular}

Instrument $16 / 16 / 2005$ 3:37:40 PM fluorous layor Table 1, Eutry 7 


\begin{tabular}{|c|c|c|c|c|c|c|}
\hline $\begin{array}{c}\text { Peak } \\
\#\end{array}$ & $\begin{array}{c}\text { RetTime } \\
\text { [min] }\end{array}$ & Type & $\begin{array}{c}\text { Width } \\
\text { [min] }\end{array}$ & $\begin{array}{c}\text { Area } \\
{\left[\mathrm{mAU}^{*} \mathrm{~s}\right]}\end{array}$ & $\begin{array}{l}\text { Height } \\
\text { [mAU] }\end{array}$ & $\begin{array}{c}\text { Area } \\
\frac{8}{8}\end{array}$ \\
\hline-- & ------ & $-1-1>$ & ------ & --------- & --------- & 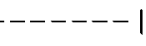 \\
\hline 10 & 9.917 & $\mathrm{BBA}$ & 0.1420 & 26.28508 & 2.84003 & 3.8609 \\
\hline Total & : & & & 680.79698 & 201.34384 & \\
\hline
\end{tabular}

Signal 3: MWD1 C, Sig=210,8 Ref=off

\begin{tabular}{|c|c|c|c|c|c|c|}
\hline $\begin{array}{c}\text { Peak } \\
\#\end{array}$ & $\begin{array}{c}\text { RetTime } \\
\text { [min] }\end{array}$ & Type & $\begin{array}{l}\text { Width } \\
{[\min ]}\end{array}$ & $\begin{array}{c}\text { Area } \\
{\left[\mathrm{mAU}^{\star} \mathrm{s}\right]}\end{array}$ & $\begin{array}{l}\text { Height } \\
\text { [mAU] }\end{array}$ & $\begin{array}{c}\text { Area } \\
\frac{8}{8}\end{array}$ \\
\hline 1 & 5.230 & $\mathrm{BB}$ & 0.0483 & 24.31178 & 7.81630 & 0.3111 \\
\hline 2 & 6.030 & BV & 0.0934 & 70.72571 & 10.13808 & 0.9049 \\
\hline 3 & 6.330 & VV & 0.0728 & 59.04247 & 11.33067 & 0.7555 \\
\hline 4 & 6.559 & VP & 0.0 & 39.9 & & 0.5116 \\
\hline 5 & 6.672 & VP & 0.0705 & 19.90493 & 3.97087 & 0.2547 \\
\hline 6 & 7.023 & VB & 0.0677 & 27.32534 & 5.72756 & 0.3496 \\
\hline 7 & 7.294 & BV & 0.0525 & 6983.33154 & 2114.91821 & 89.3526 \\
\hline 8 & & VB & & 560.36169 & 169.99718 & 7.1699 \\
\hline 9 & 8.404 & $\mathrm{BB}$ & 0.0770 & 30.49320 & 5.83002 & 0.3902 \\
\hline
\end{tabular}

Totals :

$7815.47773 \quad 2344.54785$ 


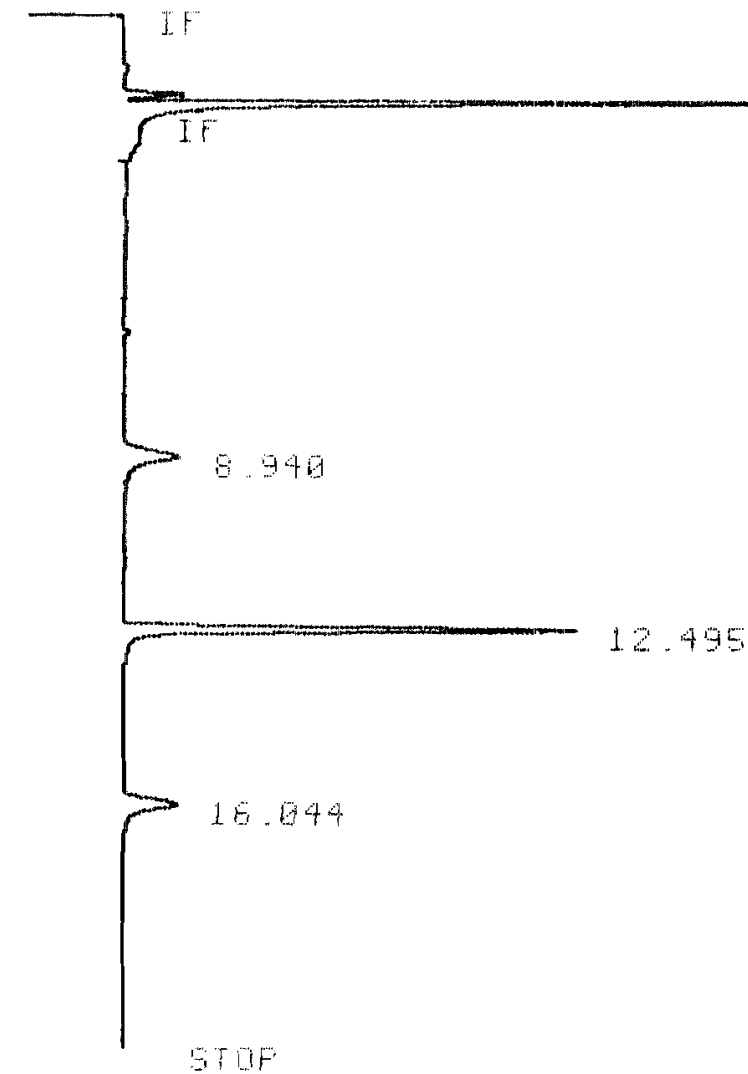

Sample \#: $\quad\left(R f_{6} \sim-11\right)_{2}^{P P h}$ FC-72 layer Notebook ref: MYO5-34 AI Initial temp: $100^{\circ} \mathrm{C}$ Initial time: $1 \mathrm{~min}$ Final temp: $250{ }^{\circ} \mathrm{C}$ Final time: $10 \mathrm{~min}$ Romp $=20^{\circ} \mathrm{C} /$ min Column: Agilient \#19091Z, HP-1, 30m x 0.32mm, 25 micron

Buth 200 Jut 9, 2064 17:84:17

SFMF'LEH

MREFI

\begin{tabular}{|c|c|c|c|c|}
\hline$F !$ & MEER & TYPE & IIOTH & AFEA \\
\hline 8.949 & 5271 & I $\mathrm{BE}$ & .295 & 94543 \\
\hline 12.495 & 17927 & $P E$ & 121 & 64.4382 \\
\hline 1. 6.94 & 4624 & 10 & 262 & 16.61994 \\
\hline
\end{tabular}

TTAL AFEA= 27B22

MUL FACTOR=1.

Table 1, Eutry 9 
RUHA 291 JUL 9, 2094 1.7:26:51

STHFT

Sample \#: $\left(\mathrm{RF}_{4} \sim \mathrm{N}_{2}\right)_{2}^{P 4} 5 \% \mathrm{H}_{2} \mathrm{O}$ cn DUF Notebook ref: MyO5-34AI

Initial temp: $100{ }^{\circ} \mathrm{C}$

Initial time: $1 \mathrm{~min}$

Final temp: $250^{\circ} \mathrm{C}$

Final time: $10 \mathrm{~min}$ Ramp $=200 \mathrm{C}$ unin

Column: Agilient \#19091Z, HP-1, 30m x 0.32mm, 25 micron

STIP

DUHस 201

J11. 9, 2004 17:26:51

HREA:

\begin{tabular}{|c|c|c|c|}
\hline $\begin{aligned} \text { ETT } \\
12+405\end{aligned}$ & FEEA & $\begin{array}{r}\text { TYFE } \\
\text { IP }\end{array}$ & $\begin{array}{r}\text { UIDTH } \\
.151\end{array}$ \\
\hline
\end{tabular}

TOTFL REEH = 1186

MUL. FACTOR=1, 0000E+BG

\section{SHMFLEH Z}

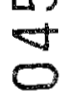



25 micron

Table 1, Eutry 10 


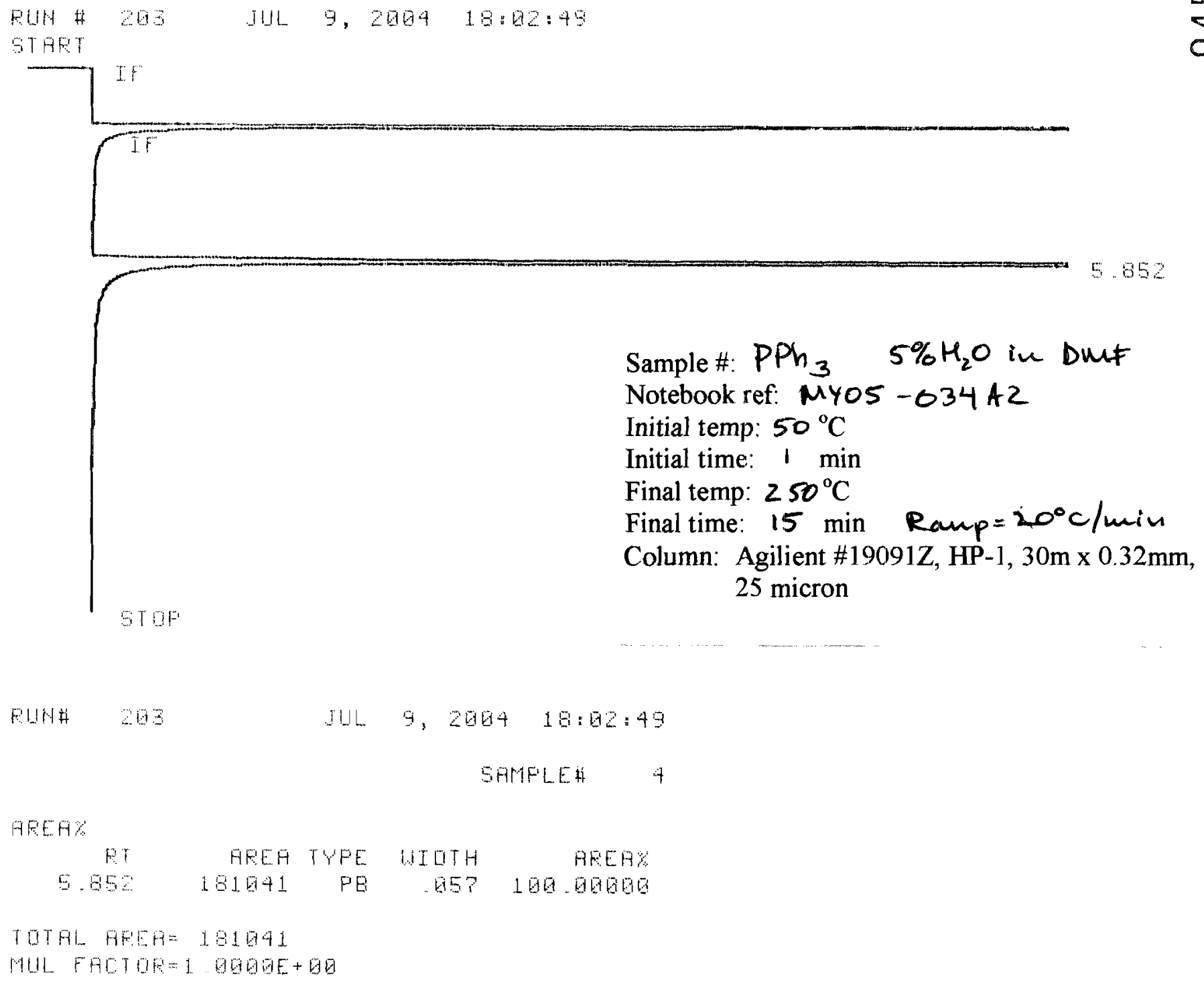

Table 1, Entry 10 
FUL

387

APF 7, 2004 17:16:36

GTAPT

I.

IF

4 organic

$\underset{\sim}{\sim}$

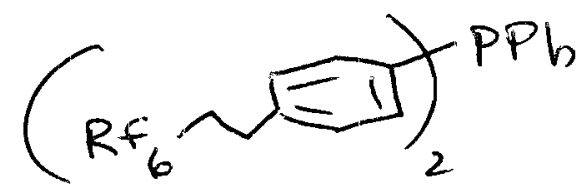

$5 \% \mathrm{H}_{2} \mathrm{O}$ in DMF

Fun 38

AFE $7,2004 \quad 17: 18: 36$

Init temp $=50^{\circ} \mathrm{C}$ SFIMLEH 23

Table 1, Entry 11

MO FUH FERKS STOREL

Init. time $=1$ min

Final time $=250^{\circ} \mathrm{C}$

Raup $=20^{\circ} \mathrm{C} /$ min

Page 53 of 126 


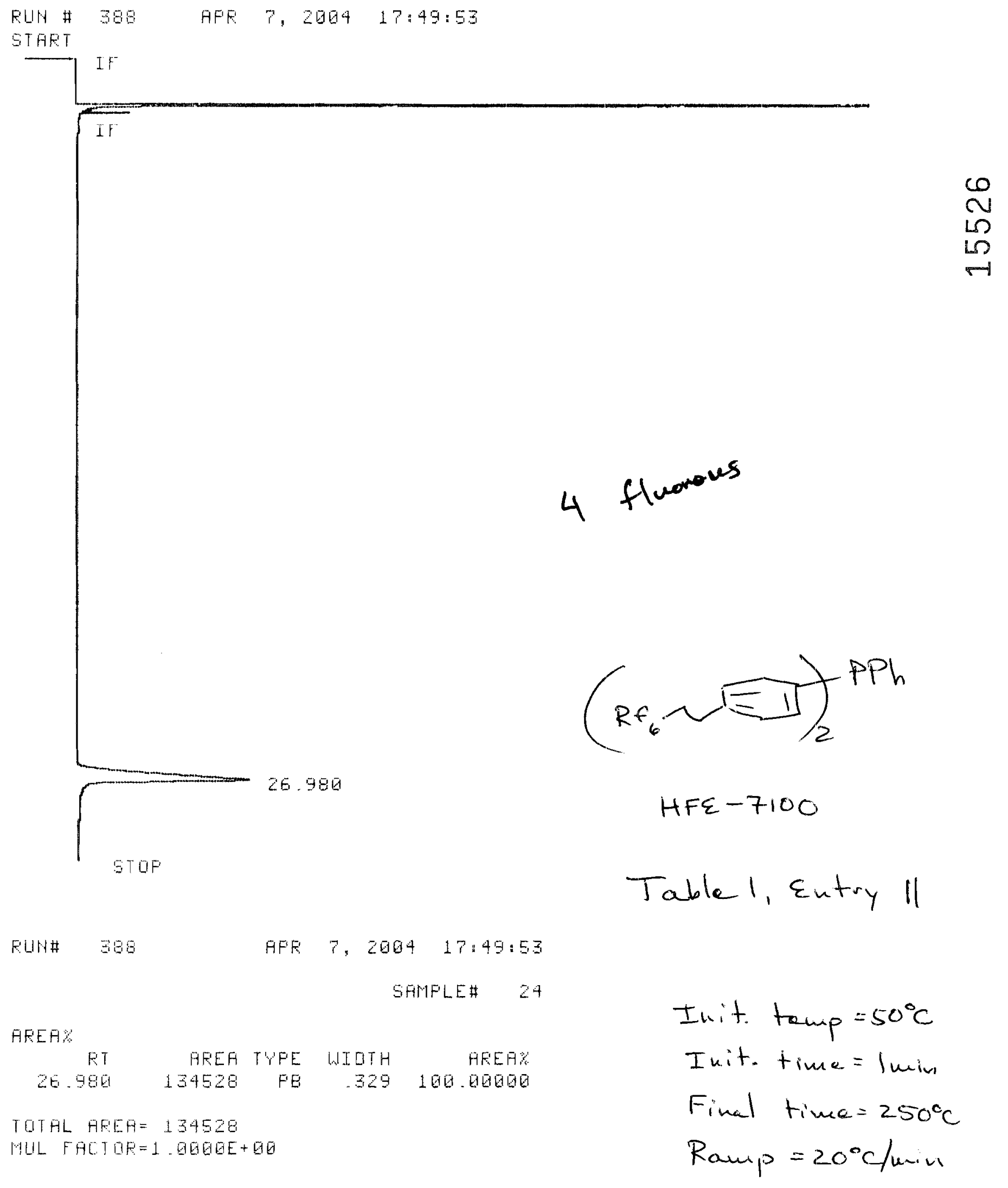




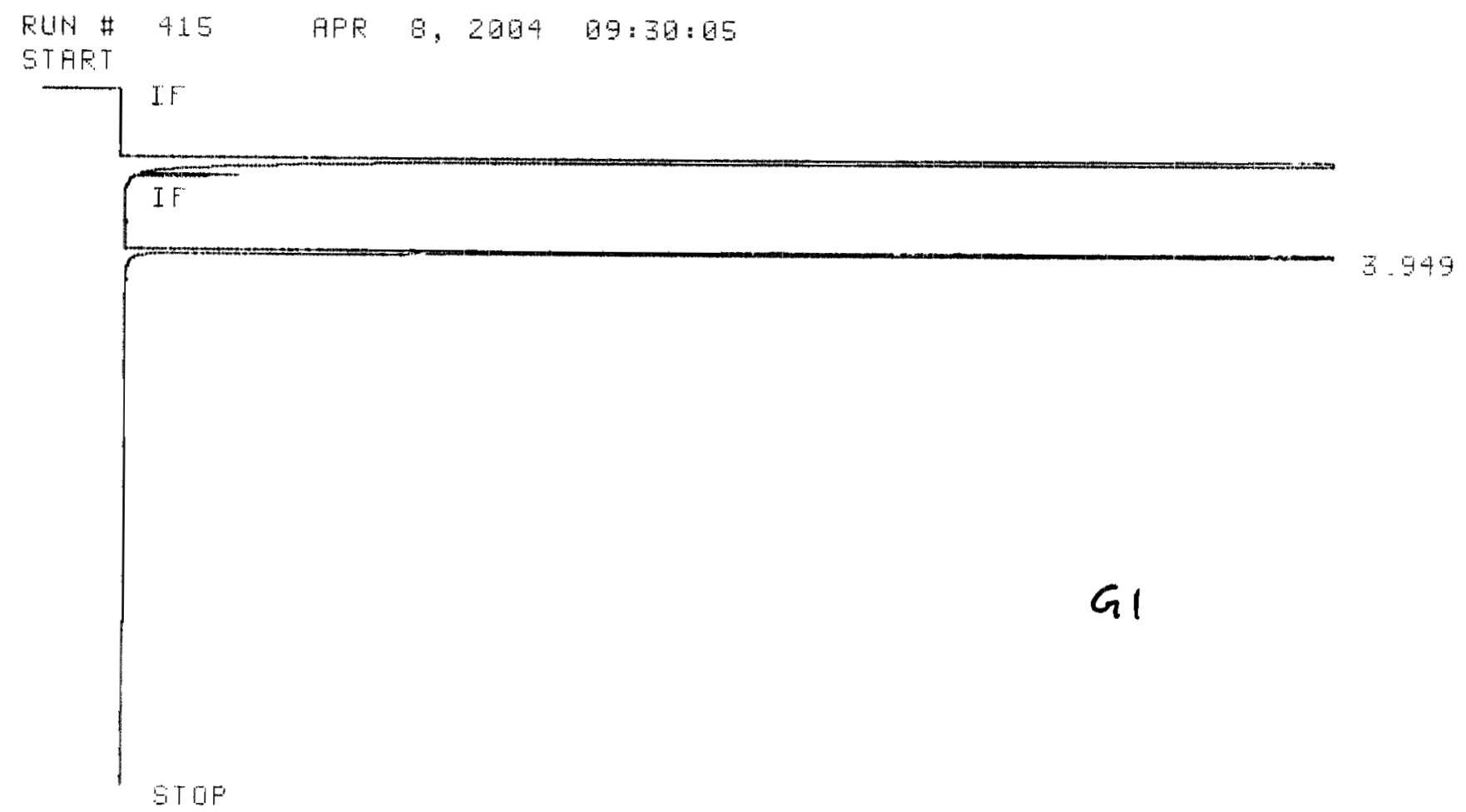

RIIHH 415

HPR $\quad$ 9. $2004 \quad 69: 30: 65$

SAMPLE\# 13

AFEF\%

\begin{tabular}{|c|c|c|c|}
\hline $\begin{array}{r}11 \\
3.949\end{array}$ & $\begin{array}{r}\text { FAER } \\
382080\end{array}$ & $\begin{array}{r}\text { TYPE } \\
\text { FE }\end{array}$ & $\begin{array}{r}. J \square T H \\
032\end{array}$ \\
\hline
\end{tabular}

TQTAL AFEA $=382082$

MUL FACTOR $=1.00 Q 0 E+Q G$

Sample \#: $\mathrm{PPh}_{3} 5 \% \mathrm{H}_{2} \mathrm{O}$ in DurF

Notebook ref: Myo4-07lal

Initial temp: $100^{\circ} \mathrm{C}$

Initial time: $1 \mathrm{~min}$

Final temp: $250{ }^{\circ} \mathrm{C}$

Table 1, Eutry 12

Final time: $15 \mathrm{~min}$ Raup $=20^{\circ} \mathrm{c} / \mathrm{min}$

Column: Agilient \#19091Z, HP-1, 30m x 0.32mm, 25 micron 


$$
\begin{aligned}
& \text { RUH \# } 416 \text { HPF } 8,2004 \text { 09:49:22 } \\
& \text { STAFT } \\
& \hline \text { IF }
\end{aligned}
$$

IF

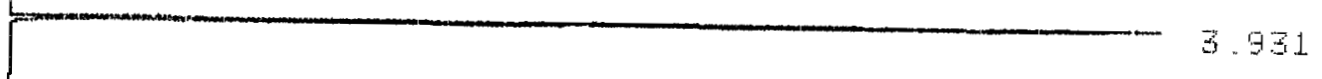

$G^{2}$

PUNA +16

FFR Q. 2014 09:49:22

SAMPLE\# 14

AFEFY

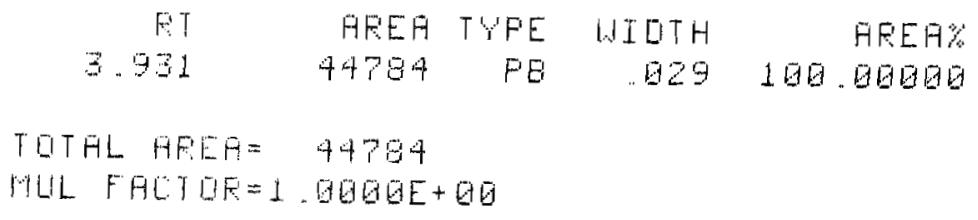
Table 1, Eutry $12 \quad \begin{aligned} & \text { Sample\#: } \mathrm{PPh}_{3} \mathrm{HAE-7100} \text { layer } \\ & \text { Notebook ref: MYO4-071G2 }\end{aligned}$

Initial temp: $100^{\circ} \mathrm{C}$

Initial time: $1 \mathrm{~min}$

Final temp: $250^{\circ} \mathrm{C}$

Final time: $15 \mathrm{~min}$ Ramp $=20^{\circ} \mathrm{C} / \mathrm{min}$

Column: Agilient \#19091Z, HP-1,30m x 0.32mm, 25 micron

Page 56 of 126 
Hating for eyetem readinese

$$
\text { FUH }
$$

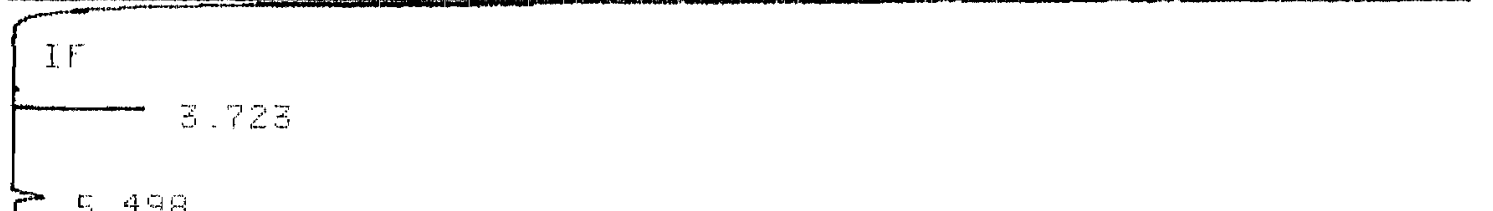

5.996

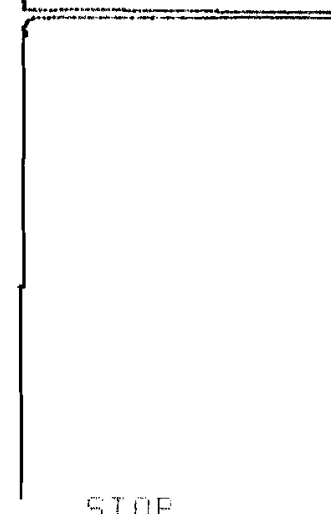

\section{MY04-070AI}

$5 T \mathrm{TF}$

Funt 39

AFE $8,2004 \quad 62: 11: 5$

SAMPLEH I

HEEFS

61
3.723
$5 .+96$
11.156

\begin{tabular}{|c|c|}
\hline$A R E F$ & TYPE \\
\hline 3605 & 80 \\
\hline 5981 & $B E$ \\
\hline 1569 & $\mathrm{~PB}$ \\
\hline
\end{tabular}
UI [ITH
620
14.1
.974

TOTHL APEF= 125216

MUL FHOJOF=1. 90QGE+

Table 2, Eutry 1

Sample \#:

Notebook ref: Myo4-070Al Initial temp: $50^{\circ} \mathrm{C}$ Initial time: $\quad \mathrm{min}$ Final temp: $250^{\circ} \mathrm{C} \quad$ Ramp $=20^{\circ} \mathrm{C} / \mathrm{min}$ Column: Agilient \#19091Z, HP-1, 30m x 0.32mm, 25 micron 


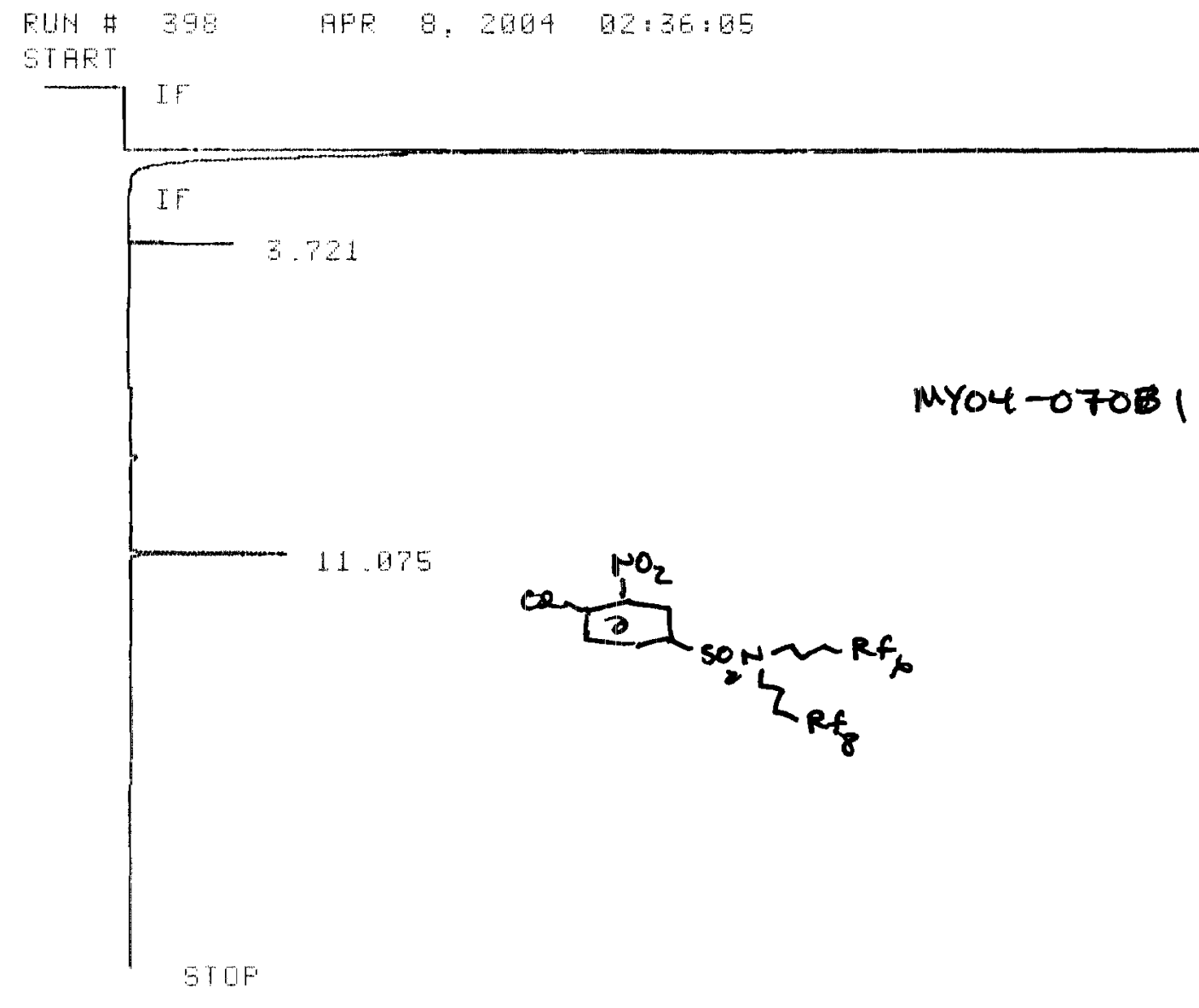

$$
\begin{aligned}
& \text { PUN\# } 998 \text { AFF 8. 2004 Q2:38:05 } \\
& \text { SFIFLE\# 2 }
\end{aligned}
$$

\begin{tabular}{|c|c|c|c|c|}
\hline $\mathrm{ET}$ & AEEA & TYPE & $11 \mathrm{OTH}$ & AREAS \\
\hline 3.721 & 3280 & $\mathrm{FE}$ & .019 & 20.53494 \\
\hline 11.875 & 12715 & $\mathrm{~PB}$ & .852 & 79.45506 \\
\hline
\end{tabular}

TITAL AFEF= 16092

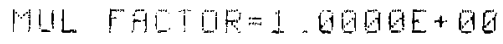

Table 2, Entry 1

Sample \#:

Notebook ref: $M 404-07 O B I$

Initial temp: $50{ }^{\circ} \mathrm{C}$

Initial time: $1 \mathrm{~min}$

Final temp: $250^{\circ} \mathrm{C}$

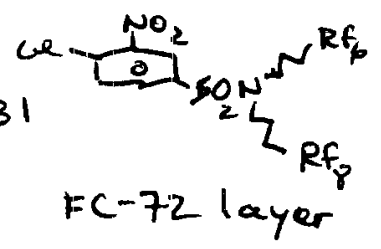

Final time: $10 \mathrm{~min} \operatorname{Ramp}=20^{\circ} \mathrm{C} / \mathrm{min}$

Column: Agilient \#19091Z, HP-1, 30m x 0.32mm, 25 micron 


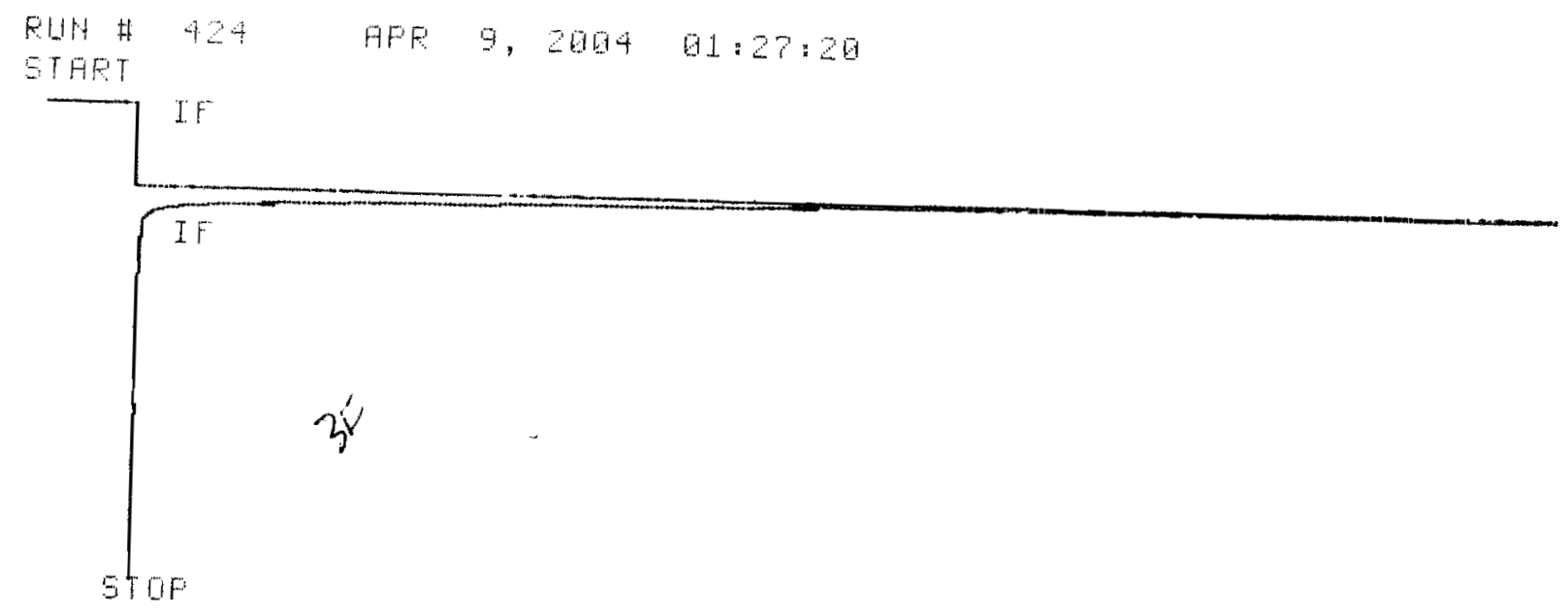

FUNA 424 AFE 9, 2004 01:20:20

$$
\text { SFMFLE }
$$

HO PUH PEFKS STOREQ

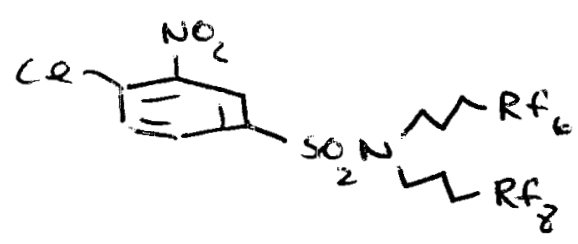

Dinf layar

Table 2, Eutry 3

$$
\begin{aligned}
& \text { Irit. temp }=250^{\circ} \mathrm{C} \\
& \text { Fincel temp }=250^{\circ} \mathrm{C}, \\
& \text { Agileut } \# 190917 . \mathrm{HP} .1 . \\
& 30 \mathrm{me} \times 0.32 \mathrm{mms}
\end{aligned}
$$




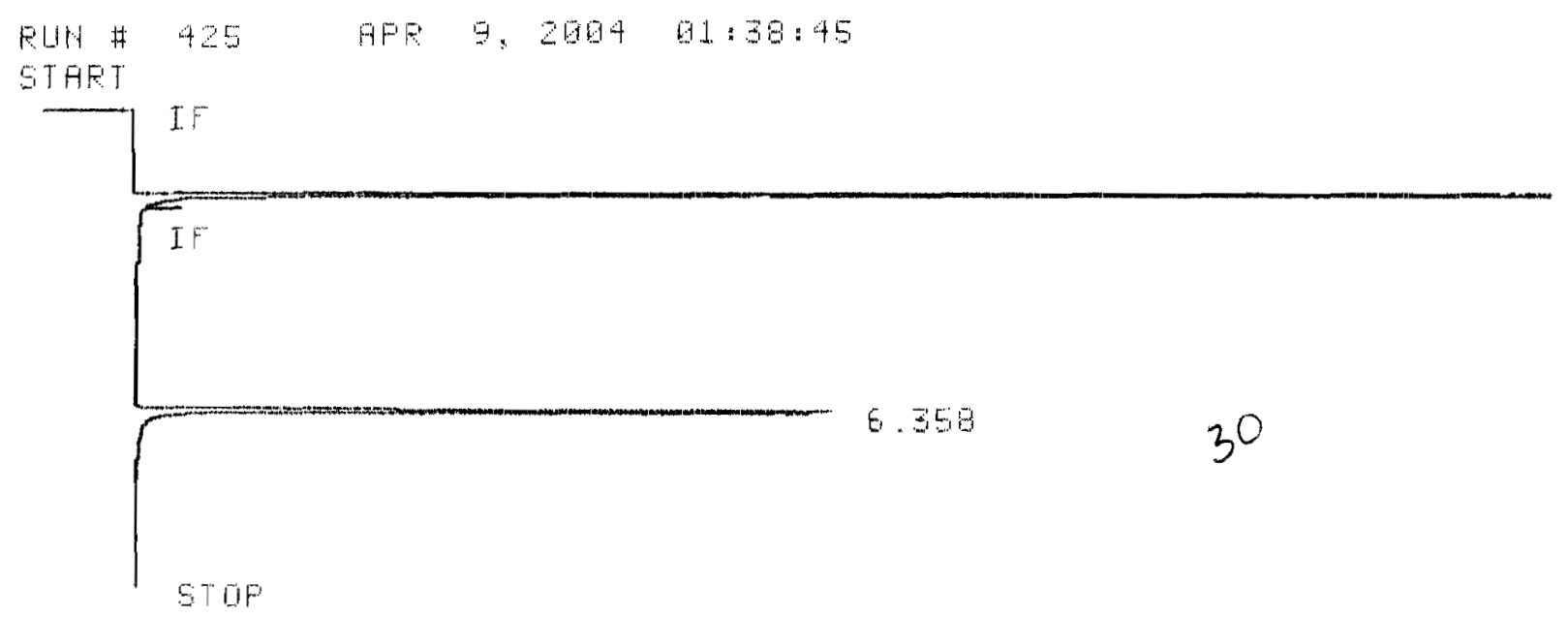

FUH\# 425 HFF 9,2964 91:39:45

SAMLE\# E

RFEFE

\begin{tabular}{|c|c|c|c|}
\hline $\mathrm{ET}$ & HEEH & TYFE & HIDTH \\
\hline
\end{tabular}

TOTAL AFEA= 5PBD?

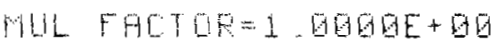

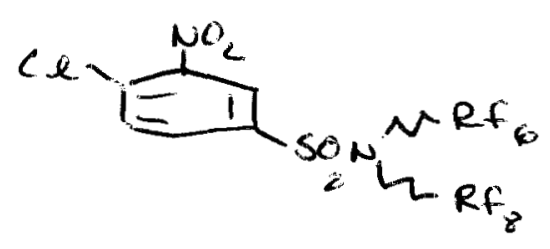

Table 2, Eutry 3

FC- 72 laya.-

$250^{\circ} \mathrm{C}$ isecratic

Agileut $\$ 19091 \mathrm{Z}, \mathrm{HP}-1$

$30 \mathrm{~m} \times 0.32 \mathrm{~mm}$ 


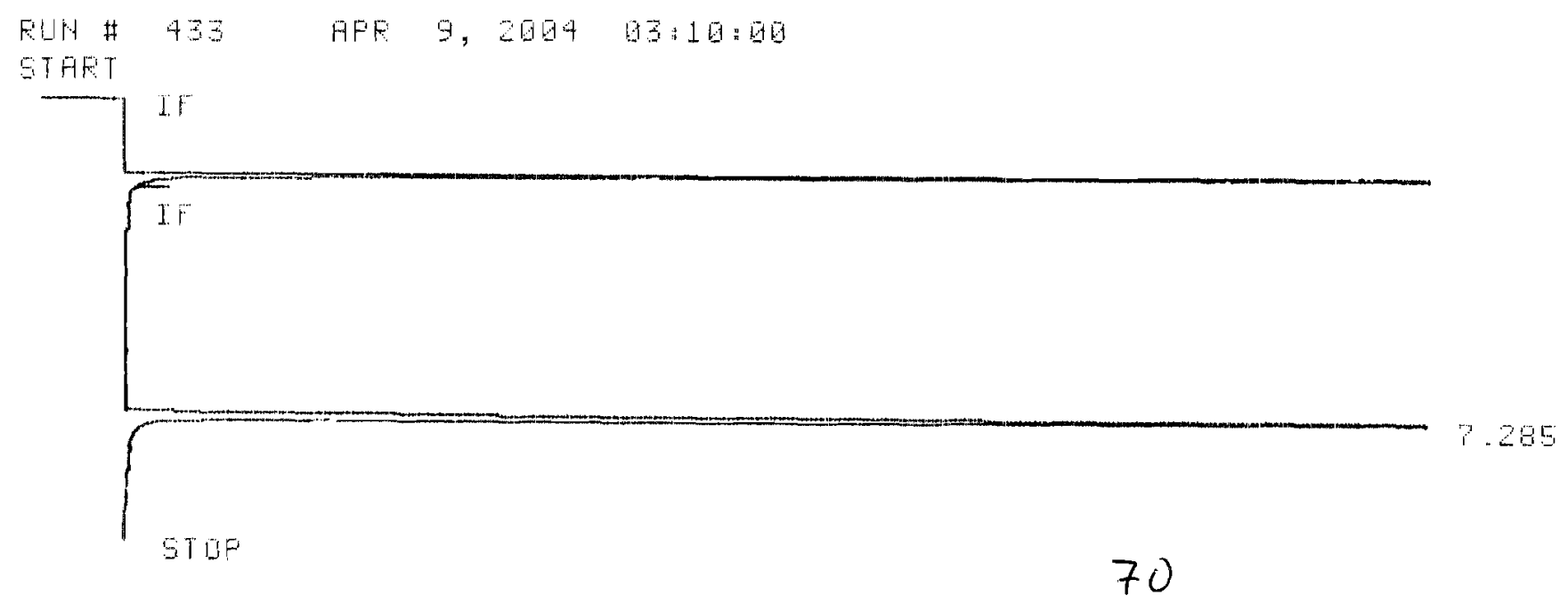

RUAH 433 AFR 9. 2984 Q3:16:80 SAMFLE\# 14

AREFH

\begin{tabular}{|c|c|c|c|c|}
\hline $\begin{array}{r}81 \\
7.285\end{array}$ & $\begin{array}{r}\text { HEEH } \\
233081\end{array}$ & $\begin{array}{r}\text { TYPE } \\
\text { PE }\end{array}$ & $\begin{array}{r}\text { UIOTH } \\
\text {. } 10 \mathrm{Z}\end{array}$ & $\begin{array}{r}\text { AREAX } \\
\text { LOQ GQGOQ }\end{array}$ \\
\hline
\end{tabular}

TOTHL AFEH= 333981

MUL FHCTOF=1. GGQGE+ GQ

$$
\begin{array}{r}
\text { DMF } \\
250^{\circ} \mathrm{C} \text { isocratic }
\end{array}
$$

Table 3, Eutry 4 


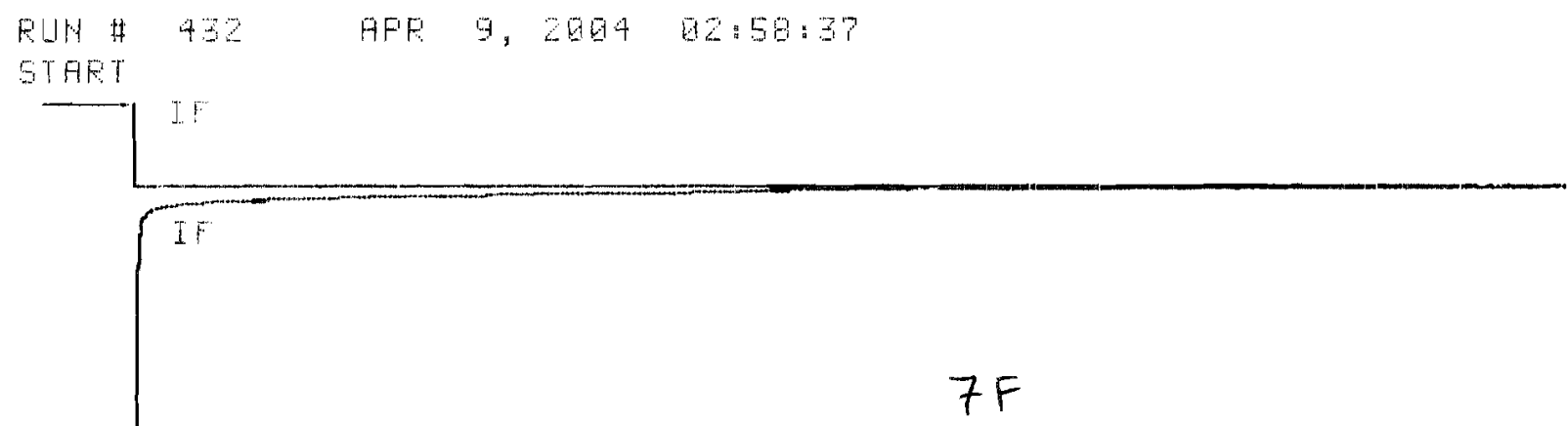

RUNH 432 HFF 9,2004 02:58:37

SFIFLE\# 13

NO FUA PEAKS STOFEO

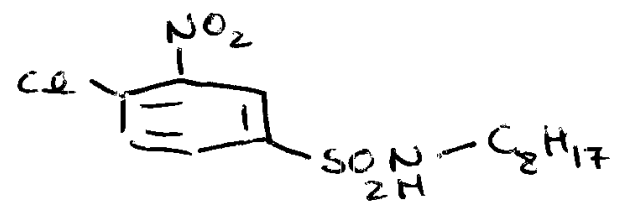

$$
\begin{aligned}
& 250^{\circ} \mathrm{C} \text { isociatic } \\
& F C-72
\end{aligned}
$$

Tabla 2, Entry 4

Page 62 of 126 


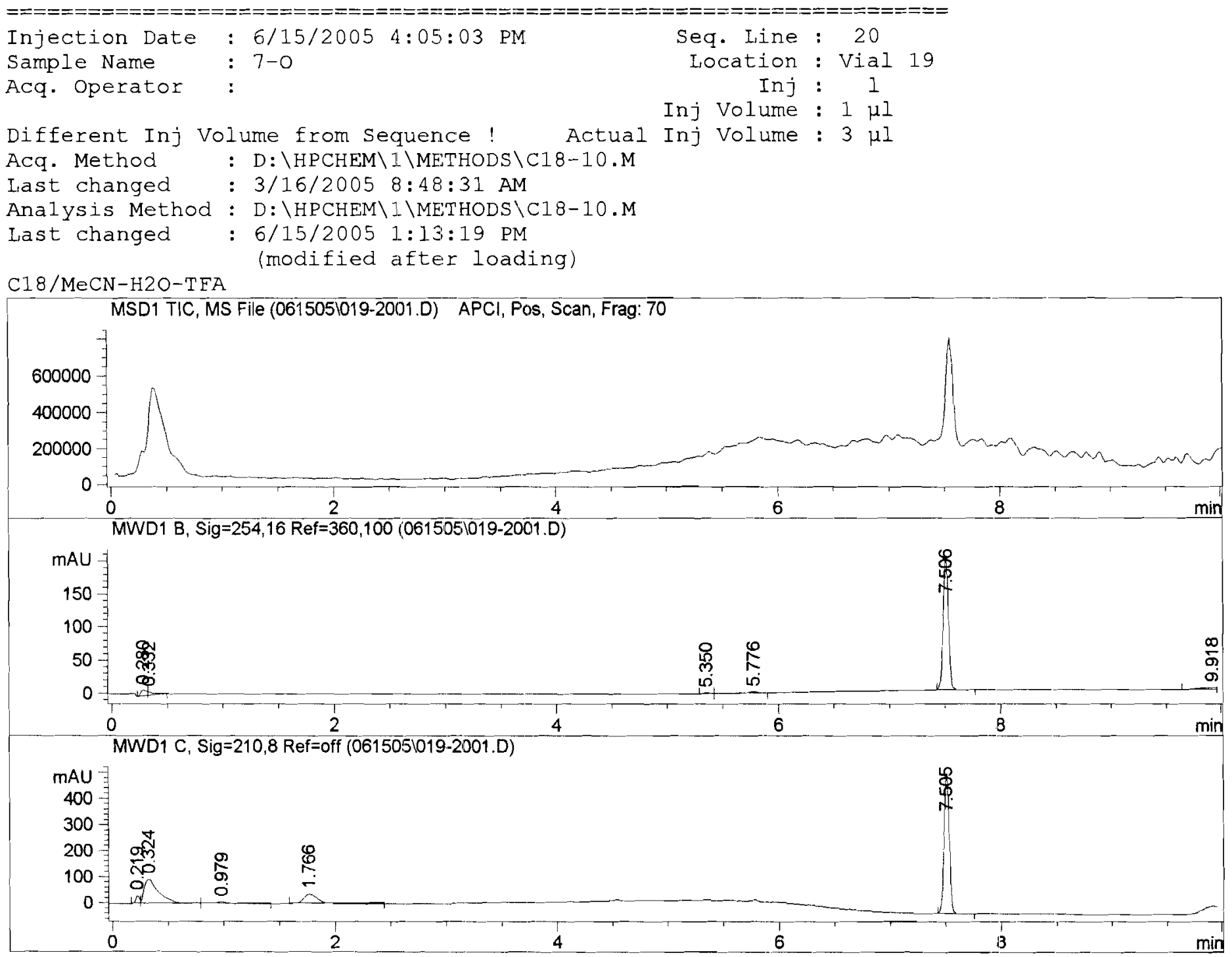

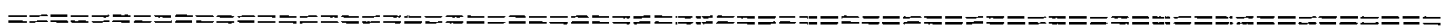

Area Percent Report

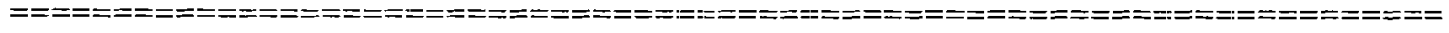

$\begin{array}{lll}\text { Sorted By } & : & \text { Signal } \\ \text { Multiplier } & : & 1.0000 \\ \text { Dilution } & : & 1.0000\end{array}$

Signal 1: MSD1 TIC, MS File

Signal 2: MWD1 B, Sig=254,16 Ref=360, 100

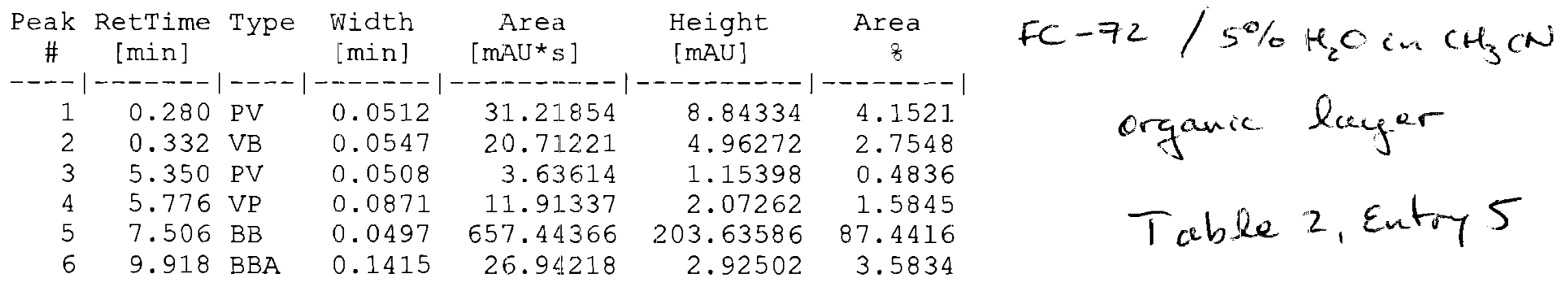

Totals :

$751.86610 \quad 223.59354$

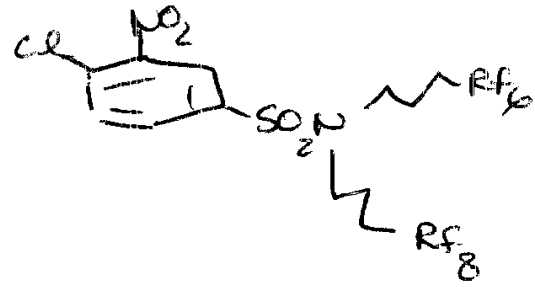

Page 63 of 126 


\begin{tabular}{|c|c|c|c|c|c|c|}
\hline $\begin{array}{c}\text { Peak } \\
\#\end{array}$ & $\begin{array}{l}\text { RetTime } \\
\text { [min] }\end{array}$ & Type & $\begin{array}{c}\text { Width } \\
\text { [min] }\end{array}$ & $\begin{array}{c}\text { Area } \\
{\left[\mathrm{mAU}{ }^{*} \mathrm{~s}\right]}\end{array}$ & $\begin{array}{l}\text { Height } \\
{[\mathrm{mAU}]}\end{array}$ & $\begin{array}{c}\text { Area } \\
\text { 8 }\end{array}$ \\
\hline-7 & $----\infty$ & & ------- & $--\cdots--\cdots---$ & --------- & ------ \\
\hline 1 & 0.219 & BV & 0.0382 & 72.02579 & 29.75432 & 2.378 .2 \\
\hline 2 & 0.324 & VV & 0.1307 & 845.50323 & 92.41065 & 27.9169 \\
\hline 3 & 0.979 & VB & 0.0958 & 49.52017 & 7.82921 & 1.6351 \\
\hline 4 & 1.766 & $\mathrm{BB}$ & 0.1362 & 31.8 .78363 & 36.38610 & 10.5256 \\
\hline 5 & 7.505 & $\mathrm{BB}$ & 0.0496 & 1742.80481 & 540.86450 & 57.5442 \\
\hline Total & Is : & & & 3028.63764 & 707.24477 & \\
\hline
\end{tabular}

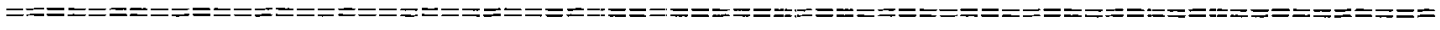

$\star \star *$ End of Report $\star \star \star$ 


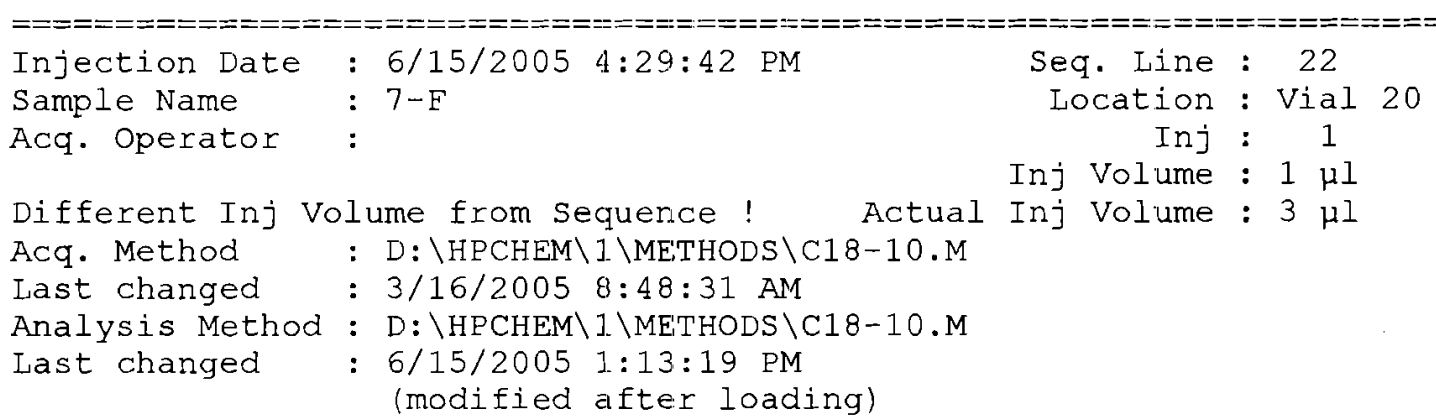

$\mathrm{C} 18 / \mathrm{MeCN}-\mathrm{H} 2 \mathrm{O}-\mathrm{TFA}$

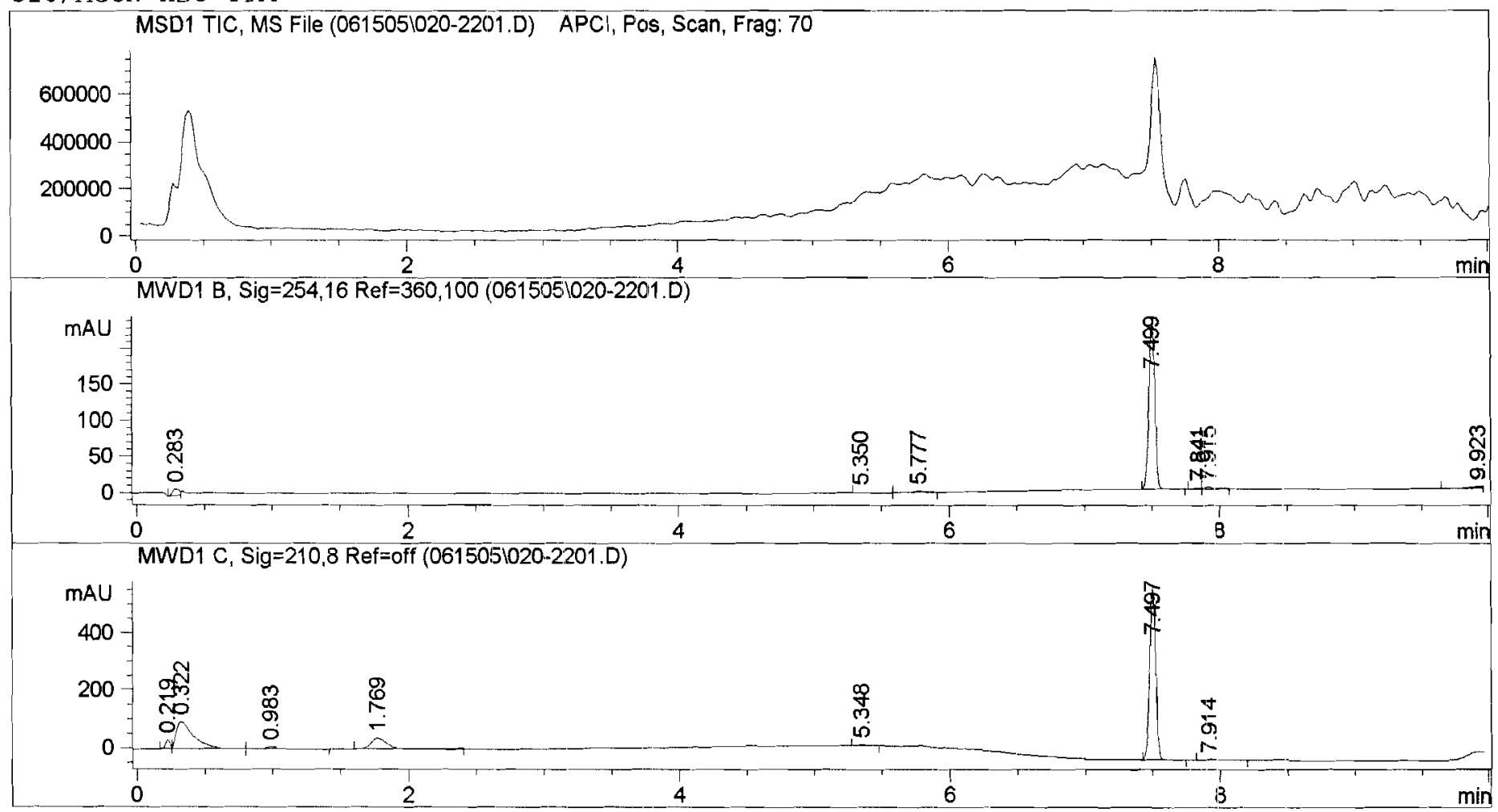

Area Percent Report

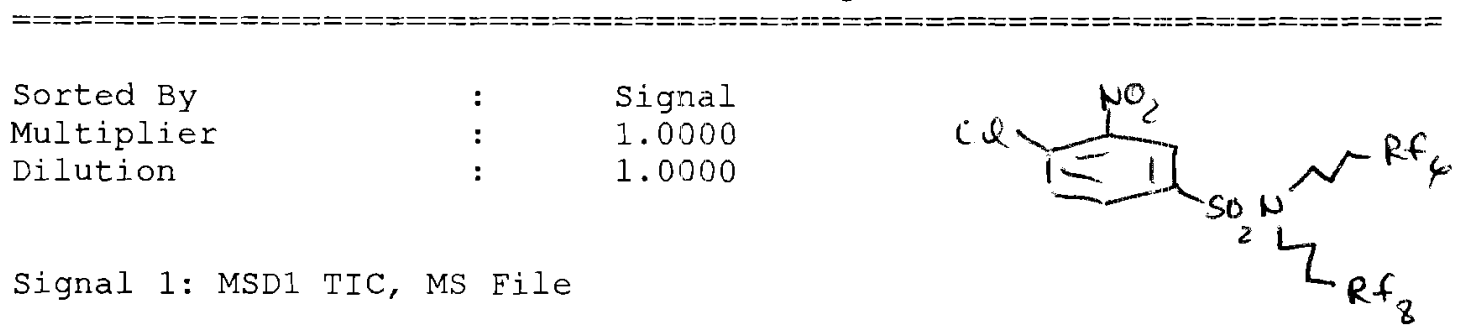

Signal 2: MWD1 B, Sig=254,16 Ref $=360,100$

Peak RetTime Type width Area Height Area

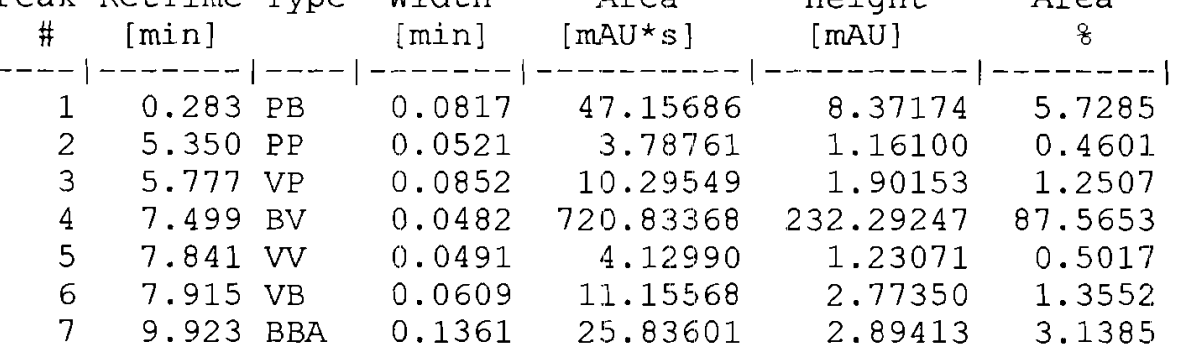

Totals :

$823.19524 \quad 250.62506$

Page 65 of 126 
Signal 3: MWD1 C, $\mathrm{Sig}=210,8$ Ref=off

\begin{tabular}{|c|c|c|c|c|c|c|}
\hline $\begin{array}{c}\text { Peak } \\
\#\end{array}$ & $\begin{array}{c}\text { RetTime } \\
\text { [min] }\end{array}$ & Type & $\begin{array}{c}\text { Width } \\
\text { [min] }\end{array}$ & $\begin{array}{c}\text { Area } \\
{\left[m A U^{*} s\right]}\end{array}$ & $\begin{array}{l}\text { Height } \\
{[\mathrm{mAU}]}\end{array}$ & $\begin{array}{c}\text { Area } \\
\frac{8}{8}\end{array}$ \\
\hline & & & & ---------- & & \\
\hline 1 & 0.219 & BV & 0.0376 & 70.10556 & 29.59752 & 2.1810 \\
\hline 2 & 0.322 & VV & 0.1296 & 845.83453 & 93.40560 & 26.3144 \\
\hline 3 & 0.983 & VB & .0928 & 48.45412 & 7.98895 & 1.5074 \\
\hline 4 & 1.769 & $\mathrm{BP}$ & 0.1340 & 32.5 .62927 & 37.98975 & 10.1305 \\
\hline 5 & 5.348 & BB & 0.0998 & 26.17635 & 3.55952 & 0.8144 \\
\hline 6 & 7.497 & $\mathrm{BB}$ & 0.0487 & 1871.91382 & 594.89062 & 58.2364 \\
\hline 7 & 7.914 & $\mathrm{BP}$ & 0.0733 & 26.22409 & 5.15921 & 0.8158 \\
\hline
\end{tabular}

Totals :

$3214.33774 \quad 772.59119$ 


\section{IF}

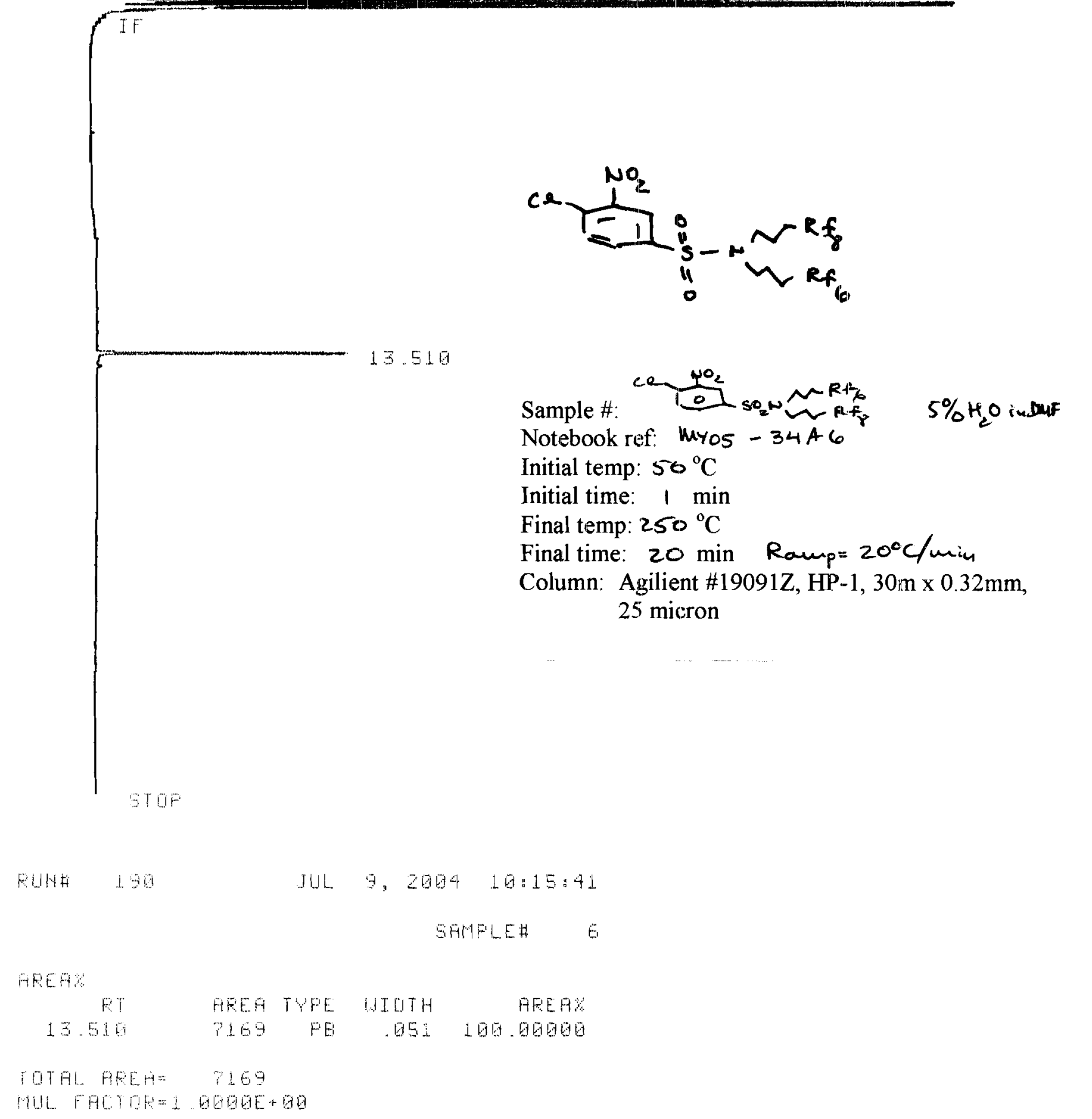

Table 2, Eutry 6 


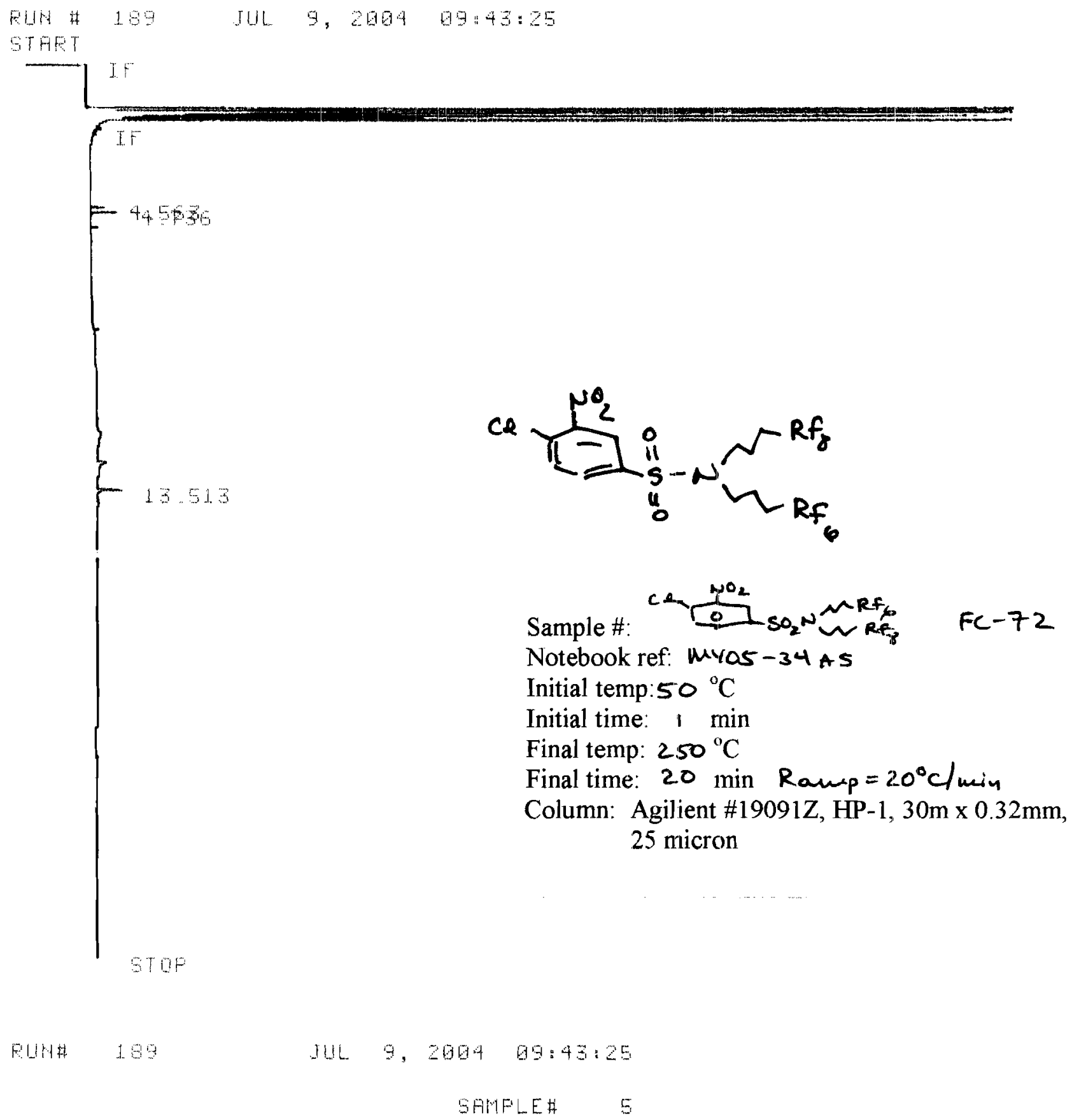

AFEAR

\begin{tabular}{|c|c|c|c|c|}
\hline $\mathrm{RT}$ & AFEA & T'PEE & MIOTH & APEF\% \\
\hline 4.563 & 192 & $F P$ & .921 & 16.69566 \\
\hline 4.736 & 286 & PE & .019 & 24.86959 \\
\hline 13.513 & 672 & BF & .452 & 58.4347 \\
\hline
\end{tabular}

TOTHL AEEA: 1150

MUL FACTOE=1 QOEUE+

table 2, Entry'o 
RUH H 268 ruL 9, 2064 20:11:41

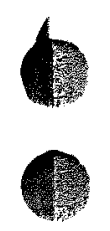

STAET

If
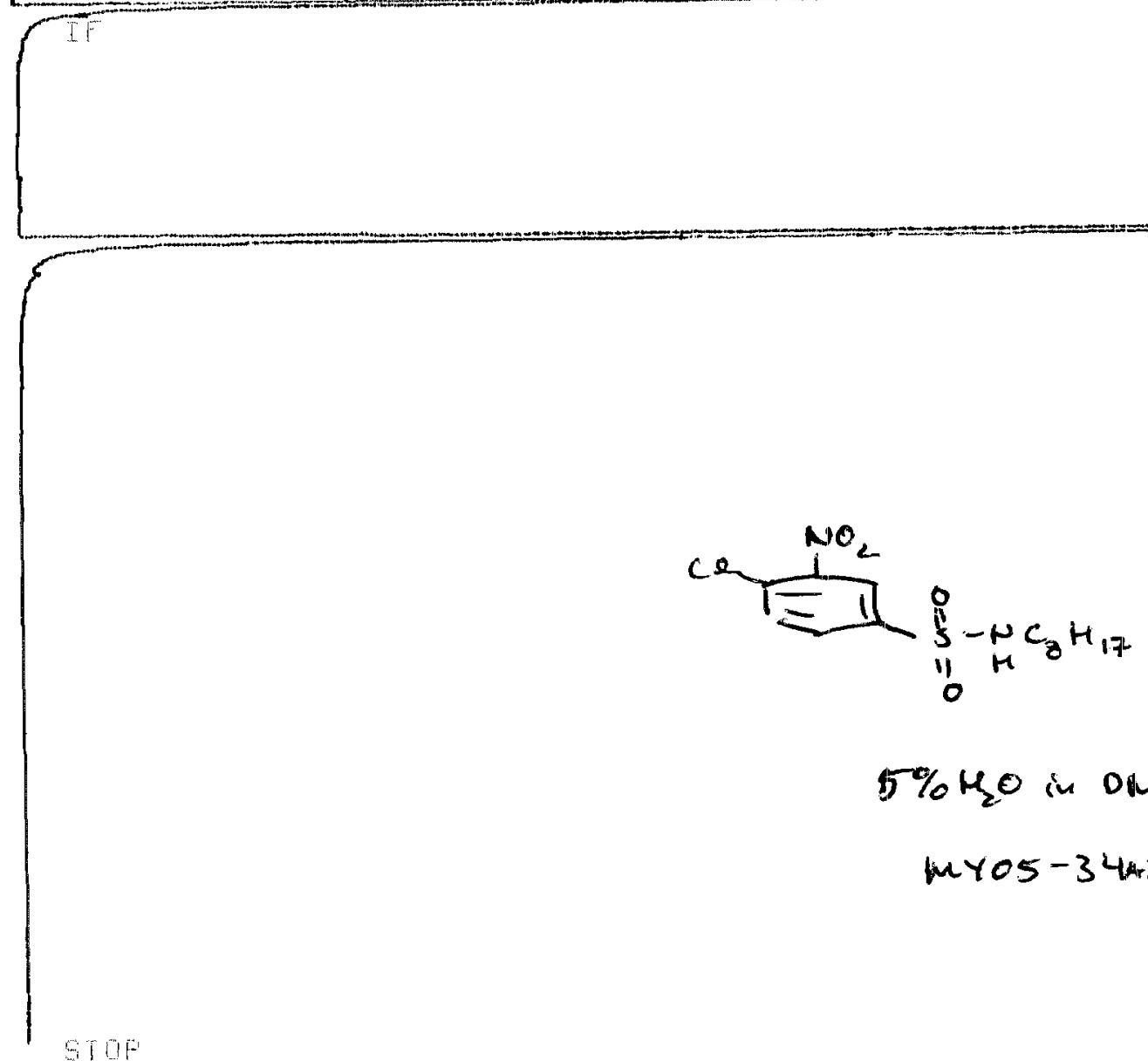

$5 \% \mathrm{H}_{2} \mathrm{O}$ in ORHF

my05-344.8

F!ll 296

IUL $9,2004 \quad 20: 11: 41$

SATIFLEH

FEEA\%

\begin{tabular}{|c|c|c|c|}
\hline & AFEH & $\begin{array}{r}\text { TYPE } \\
P F_{1}\end{array}$ & $\begin{array}{r}\text { WL L I H } \\
976\end{array}$ \\
\hline
\end{tabular}

TOTHL. AEEA = 13486

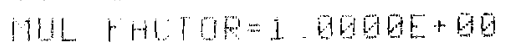

Table 2, Eutry 7 


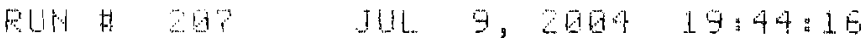

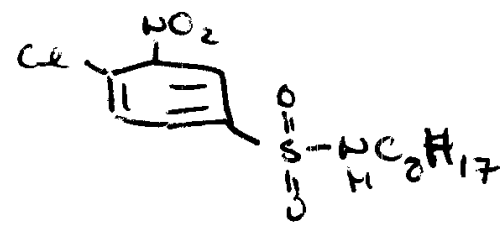

$\mathrm{FC}-72$

$\operatorname{my} 405-34 A 87$

$$
\text { Table 2, Eutry } 7
$$




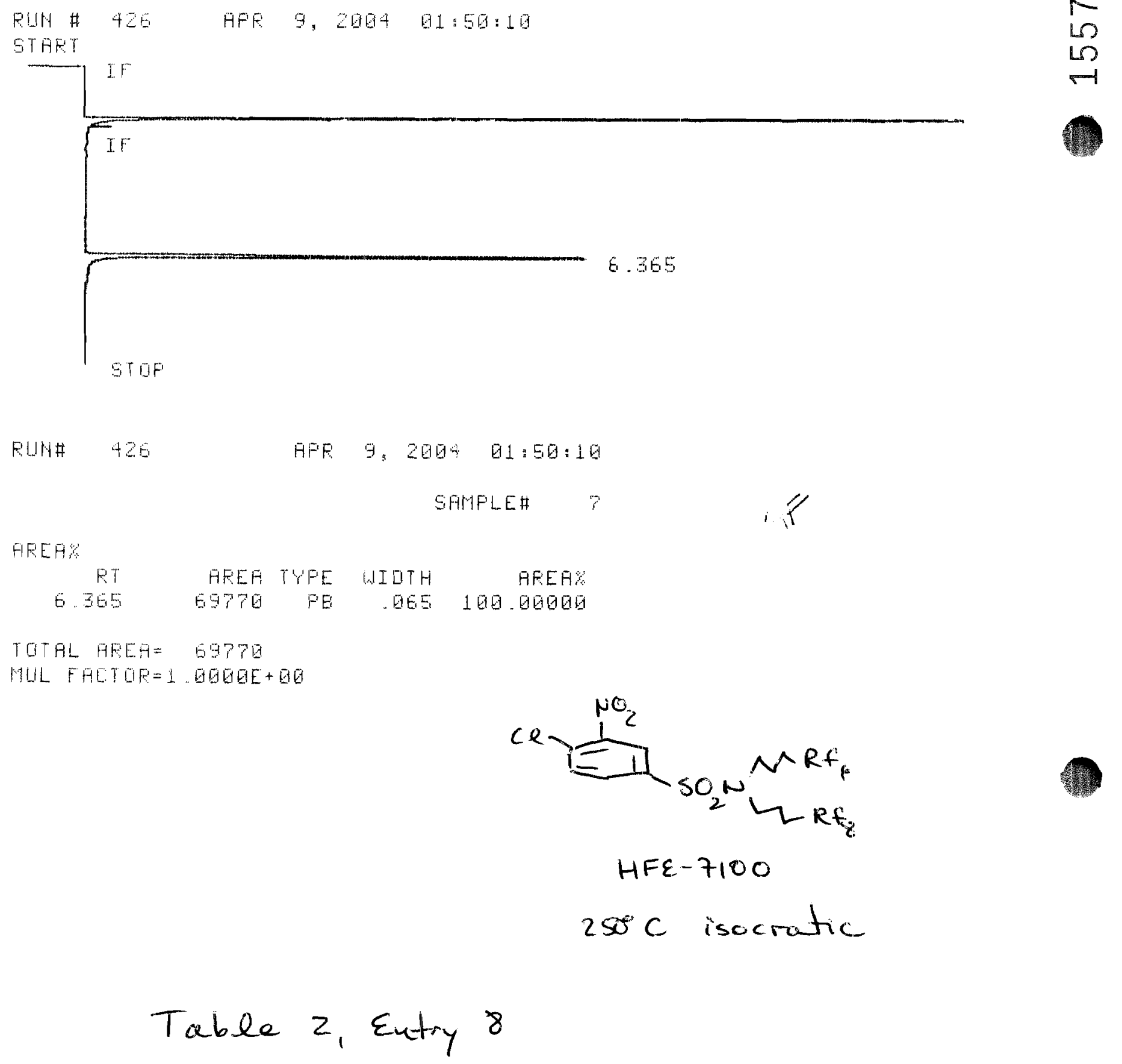

RUHA 426 HFE 9,200\% 01:50:10

\begin{tabular}{|c|c|c|c|c|}
\hline & & & IDTH & \\
\hline 6.365 & 69770 & $\mathrm{PE}$ & QES & 100.00000 \\
\hline
\end{tabular}

TOTAL AEEG = 6977日

HUL FACTOR=1. GOQUE+QG 

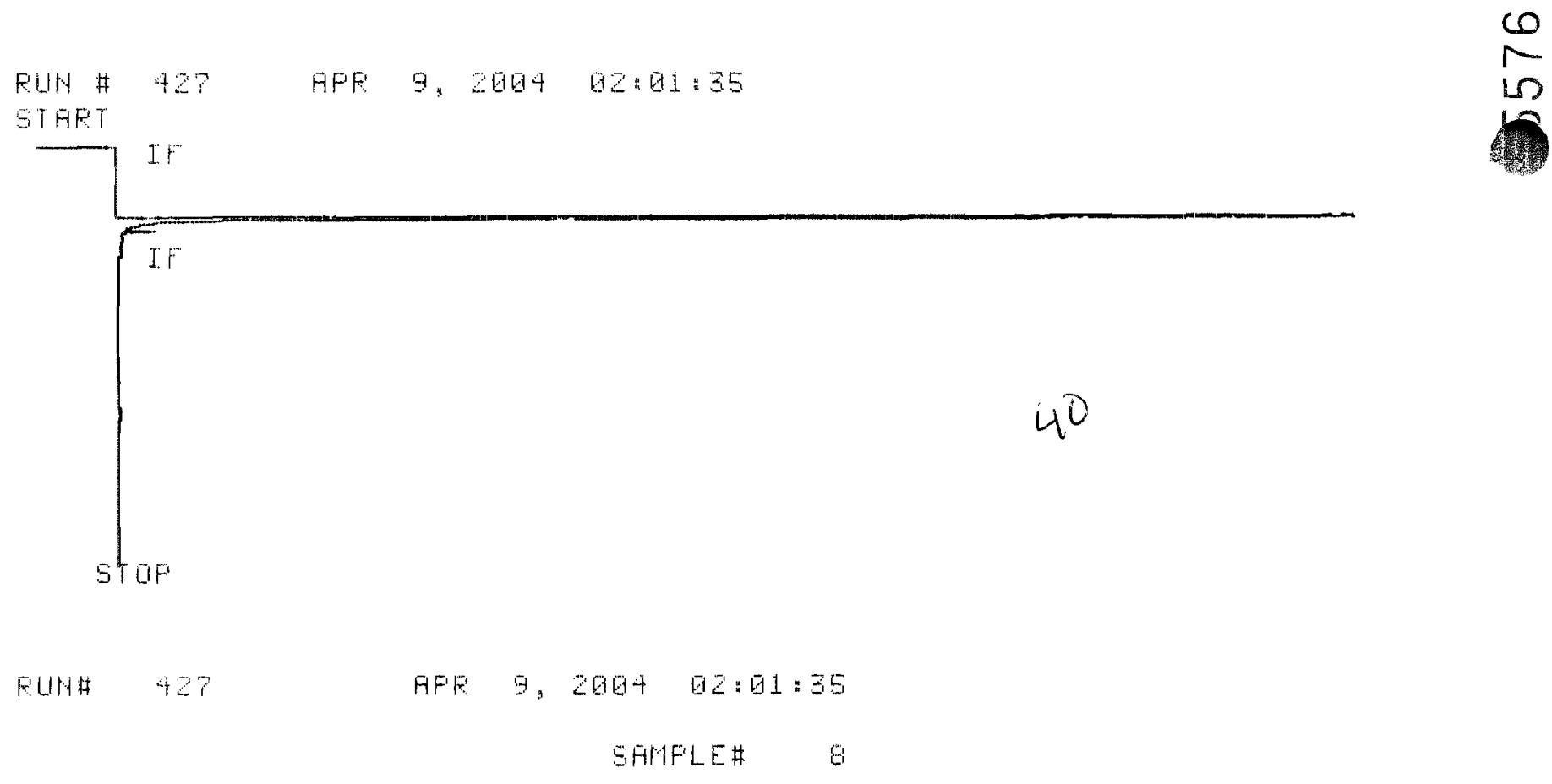

HO FUH FEAKS GTUEE

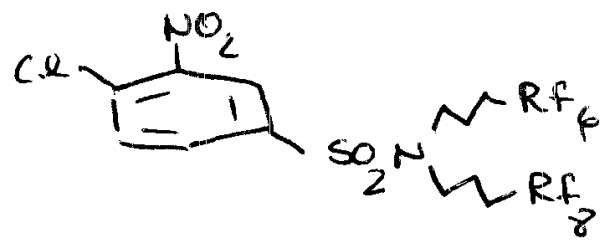

$250^{\circ} \mathrm{C}$ isocratic $5 \% \mathrm{H}_{2} \mathrm{O}$ in DMF

Table 2, Eutry 8

Page 72 of 126 


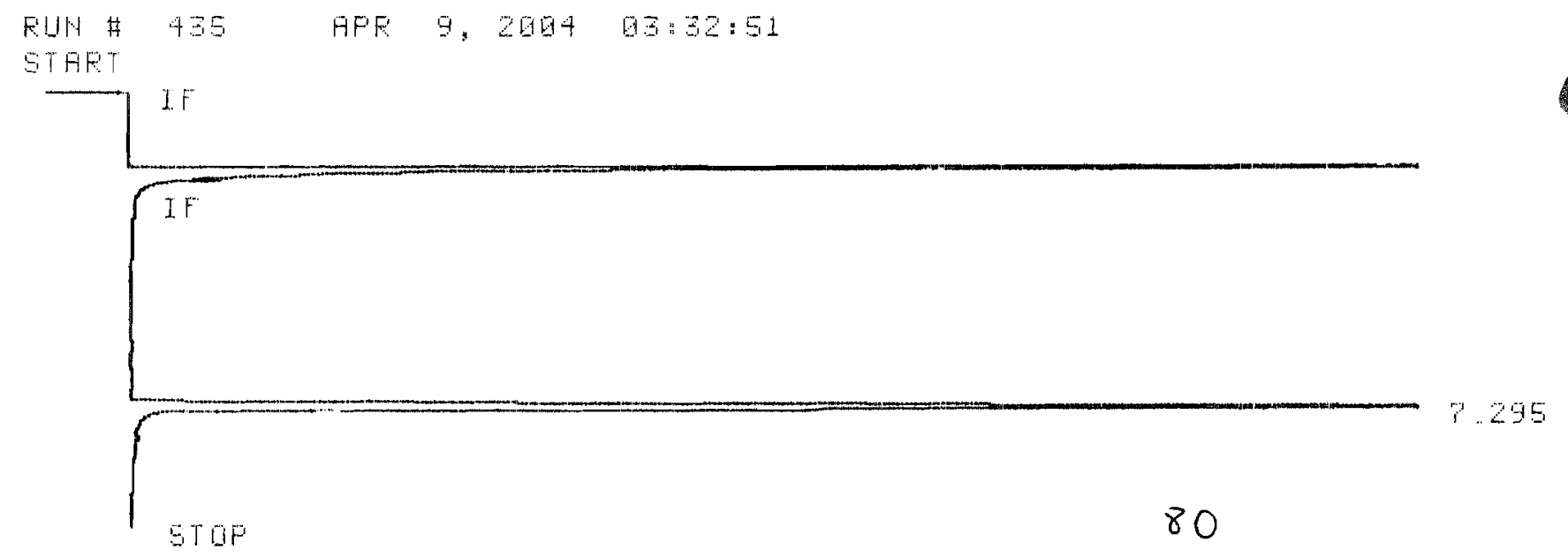

Fulf 455

$$
\begin{array}{r}
\text { HFF } 9,2064 \text { 03:32:51 } \\
\text { SHMFLE } 16
\end{array}
$$

FFEAB

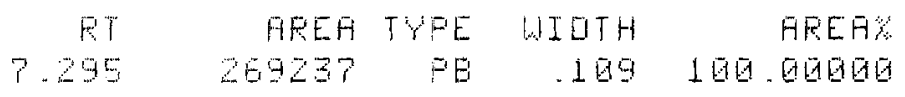

TOTHL AEEA = 26923?

NUL FACTOE=1. GOEGE+QD

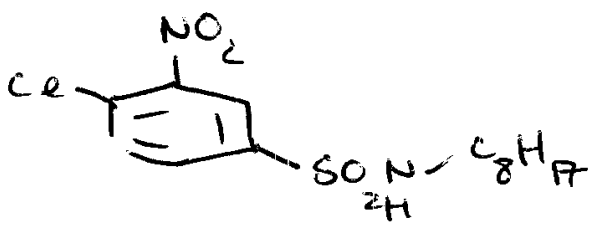

$5 \% \mathrm{H}_{2} \mathrm{O}$ in DMF

$250^{\circ} \mathrm{C}$ isocaratic

\section{Table 2, Eutry 9}




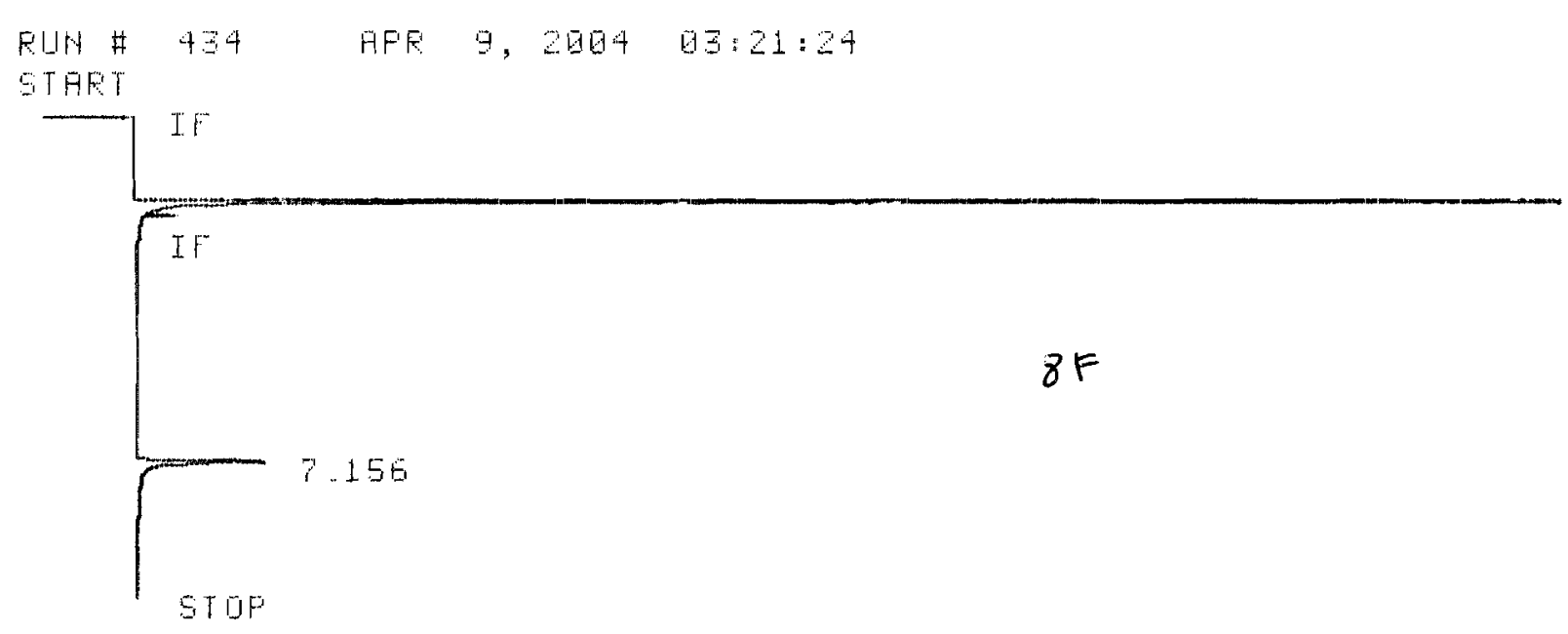

FUNH 434

$$
\begin{array}{r}
\text { HFR } 9,2064 \text { Q } 2: 21: 24 \\
\text { SFMPLEH } 15
\end{array}
$$

AREAS

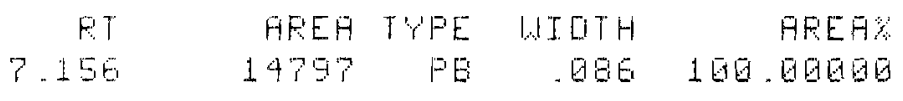

TOTAL AFEA $=14797$

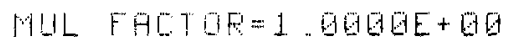

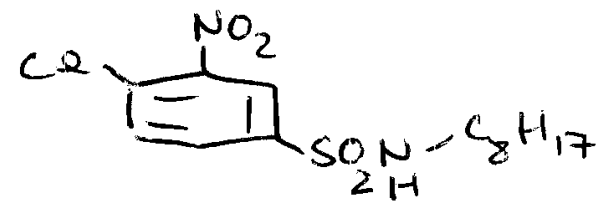

$H F \varepsilon-7100$

$250^{\circ} \mathrm{C}$ isocratic

Table 2, Eutry 9

Page 74 of 126 


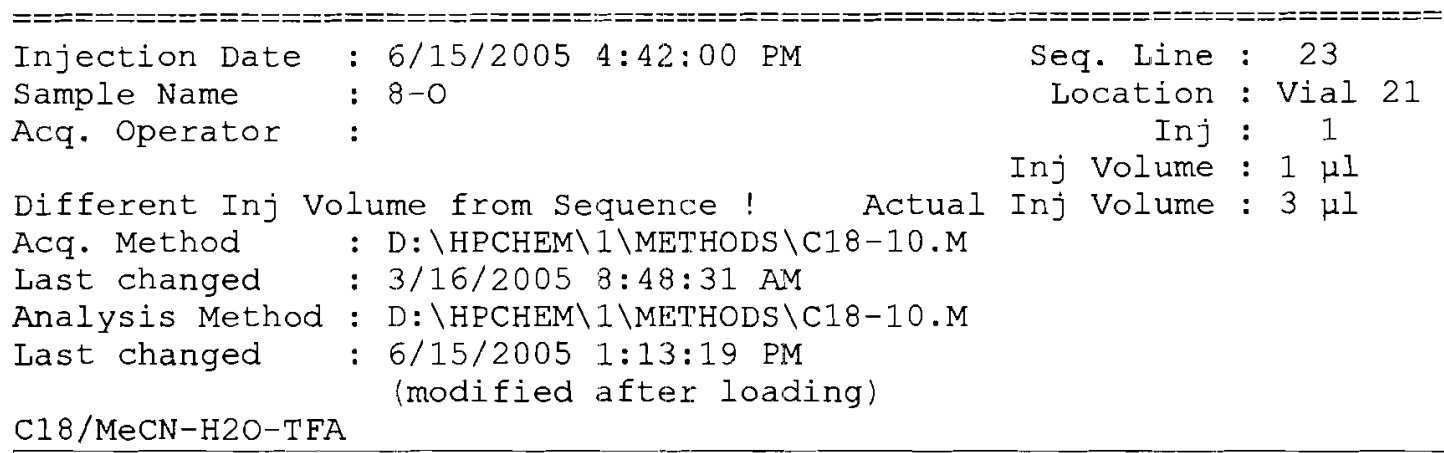

$\mathrm{C} 18 / \mathrm{MeCN}-\mathrm{H} 2 \mathrm{O}-\mathrm{TFA}$

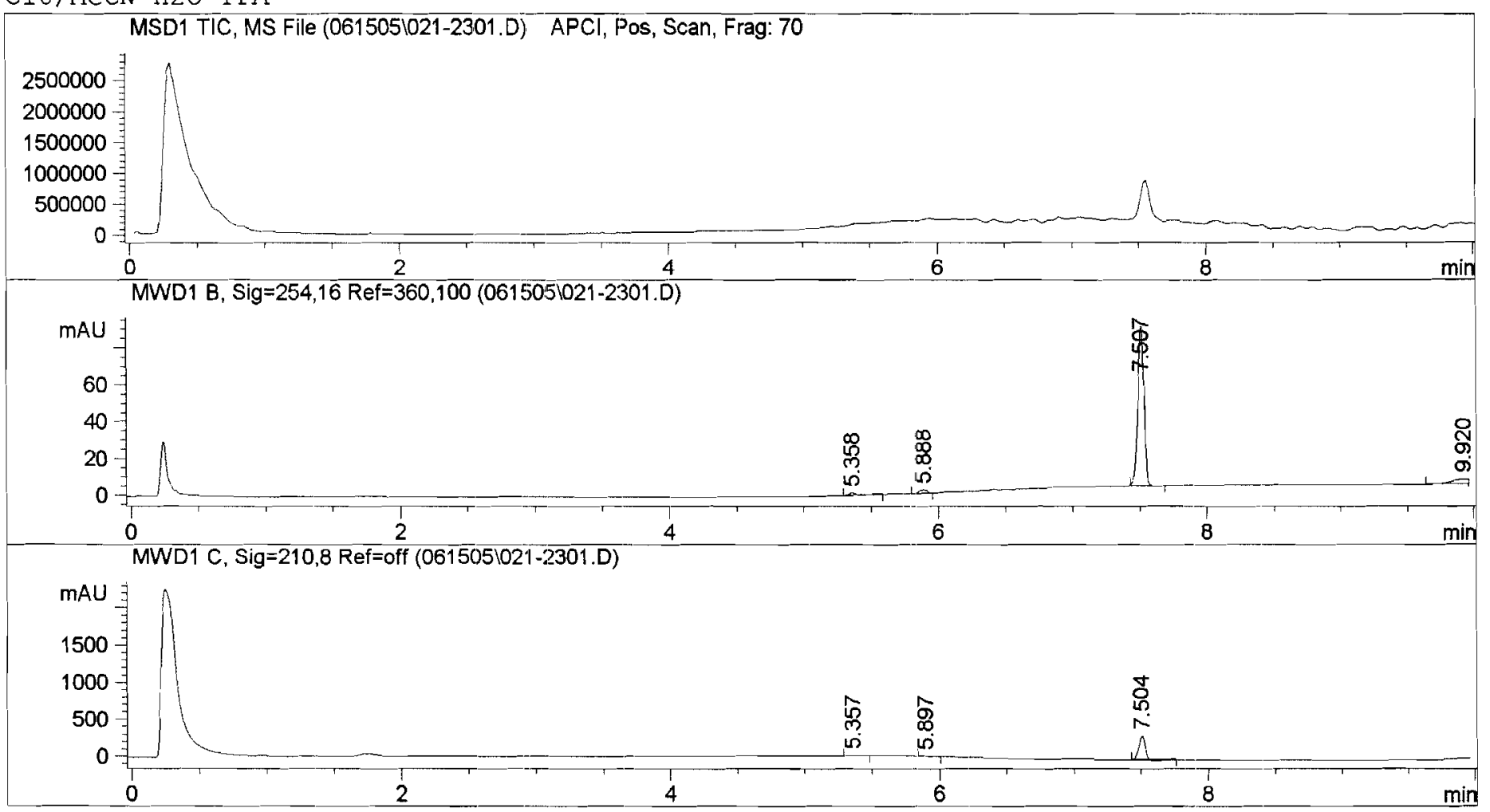

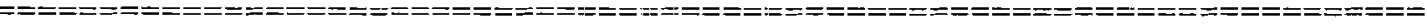

Area Percent Report

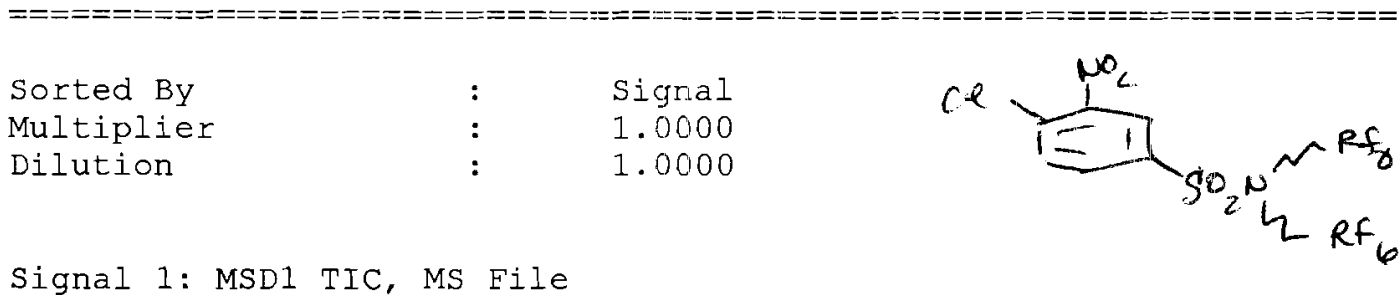

Signal 2: MWD1 B, Sig=254,16 Ref $=360,100$

$2: F C-72: H F E-7100 / 5 \% \mathrm{H}_{2} \mathrm{O}$ in DH.

\begin{tabular}{|c|c|c|c|c|c|c|}
\hline $\begin{array}{c}\text { Peak } \\
\text { \# }\end{array}$ & $\begin{array}{c}\text { RetTime } \\
\text { [min] }\end{array}$ & Type & $\begin{array}{l}\text { Width } \\
\text { [min] }\end{array}$ & $\begin{array}{c}\text { Area } \\
{\left[\mathrm{mAU}^{*} \mathrm{~s}\right]}\end{array}$ & $\begin{array}{l}\text { Height } \\
\text { [mAU] }\end{array}$ & $\begin{array}{c}\text { Area } \\
\frac{8}{8}\end{array}$ \\
\hline 1 & & $P P$ & 0.0485 & 4.4 & 1.4 & 41 \\
\hline 2 & 888 & $P P$ & 0.0670 & 085 & 1.97893 & 2.5183 \\
\hline 3 & & $\mathrm{BB}$ & 0.0515 & 277.58072 & 86.38694 & 87.6997 \\
\hline 4 & 9.920 & $\mathrm{BBA}$ & 0.1411 & 26.54861 & 2.89140 & 8.3879 \\
\hline
\end{tabular}

organic lager

Totals :

$316.51267 \quad 92.66591$

Table 2, Eutry 10 


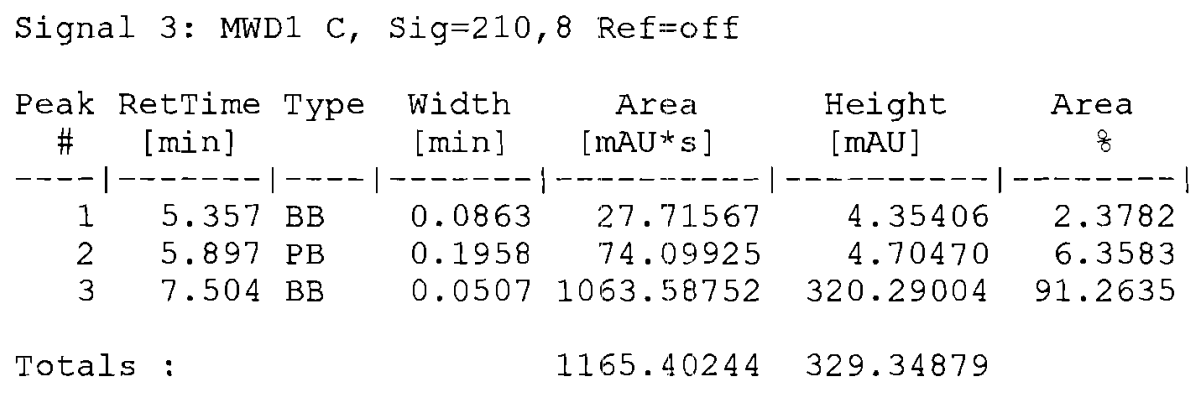

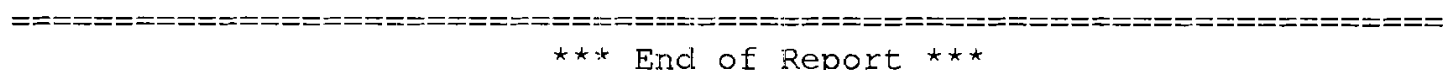




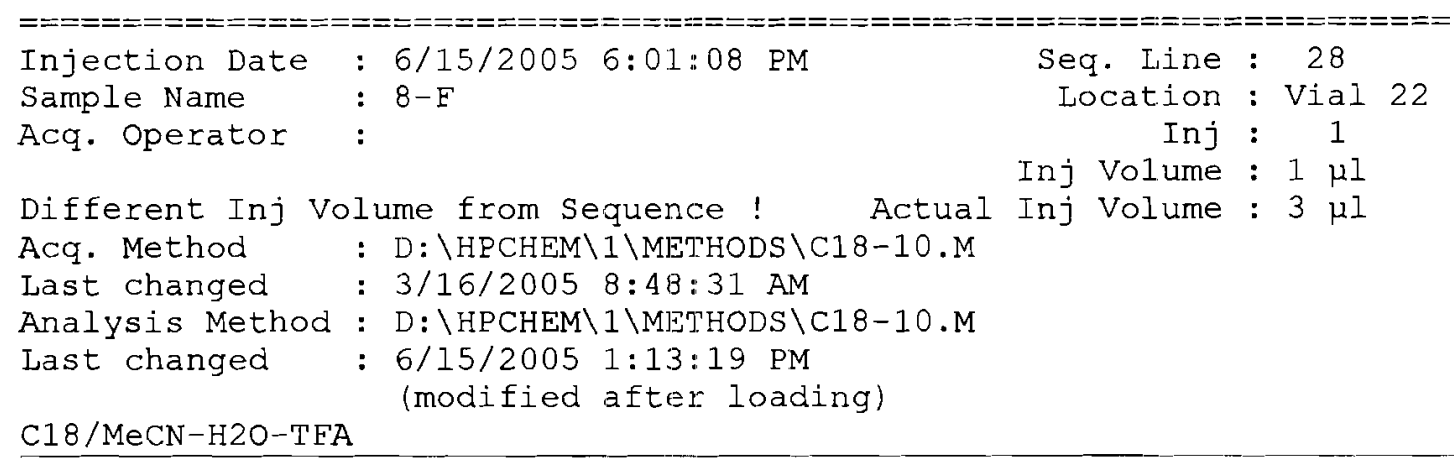

$\mathrm{C} 18 / \mathrm{MeCN}-\mathrm{H} 2 \mathrm{O}-\mathrm{TFA}$

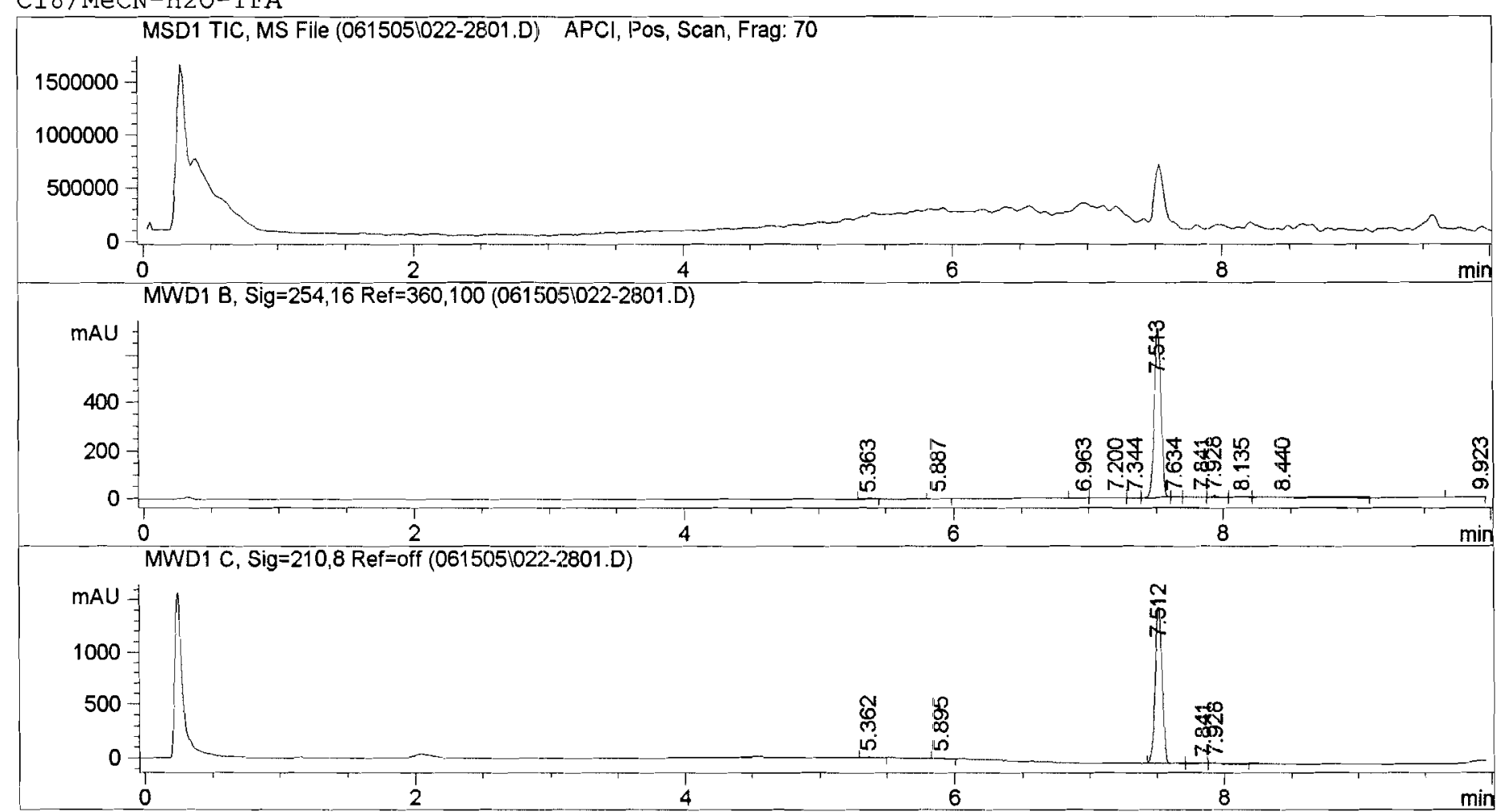

Area Percent Report

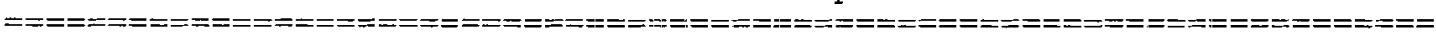

$\begin{array}{lll}\text { Sorted By } & : & \text { Signal } \\ \text { Multiplier } & : & 1.0000 \\ \text { Dilution } & : & 1.0000\end{array}$

Signal 1: MSDl TIC, MS File

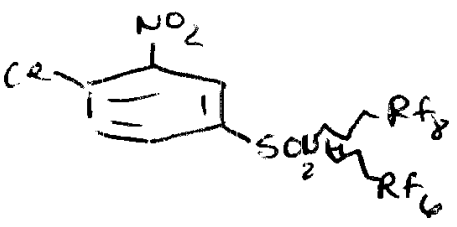

Signal 2: MWD1 B, Sig=254,16 Ref=360, 100

\begin{tabular}{|c|c|c|c|c|c|c|}
\hline $\begin{array}{c}\text { Peak } \\
\#\end{array}$ & $\begin{array}{c}\text { RetTime } \\
\text { [min] }\end{array}$ & Type & $\begin{array}{l}\text { Width } \\
\text { [min] }\end{array}$ & $\begin{array}{c}\text { Area } \\
{\left[m A U^{*} s\right]}\end{array}$ & $\begin{array}{l}\text { Height } \\
\text { [mAU] }\end{array}$ & $\begin{array}{c}\text { Area } \\
8\end{array}$ \\
\hline & & & & & & \\
\hline 1 & 5.363 & PP & 0.0520 & 5.32635 & 1.63510 & 0.2047 \\
\hline 2 & 5.887 & PP & 0.0701 & 7.95154 & 1.93027 & 0.3056 \\
\hline 3 & 6.963 & BV & 0.0749 & 3.45490 & $6.41018 \mathrm{e}-1$ & $0.132 B$ \\
\hline 4 & 7.200 & VV & 0.1363 & 10.90298 & 1.03985 & 0.4190 \\
\hline 5 & 7.344 & VV & 0.0694 & 6.25386 & 1.31627 & 0.2404 \\
\hline 6 & 7.513 & VV & 0.0561 & 2449.43774 & 712.91217 & 94.1417 \\
\hline 7 & 7.634 & VV & 0.0610 & 9.77844 & 2.32636 & 0.3758 \\
\hline 8 & 7.841 & VV & 0.0788 & 21.79629 & 3.80514 & 0.8377 \\
\hline 9 & 7.928 & VV & 0.0652 & 31.62836 & $\begin{array}{c}7.21162 \\
\text { Page } 77 \text { of } 12\end{array}$ & 1.2156 \\
\hline
\end{tabular}

fluovous loper

Table 2, Eutry 10 
Data File D: \HPCHEM $\backslash \backslash D A T A \backslash 061505 \backslash 022-2801 . D$

\begin{tabular}{|c|c|c|c|c|c|c|}
\hline $\begin{array}{c}\text { Peak } \\
\#\end{array}$ & $\begin{array}{c}\text { RetTime } \\
\text { [min] }\end{array}$ & Type & $\begin{array}{l}\text { Width } \\
\text { [min] }\end{array}$ & $\begin{array}{c}\text { Area } \\
{\left[\mathrm{mAU}^{\star} \mathrm{s}^{-}\right]}\end{array}$ & $\begin{array}{l}\text { Height } \\
\text { [mAU] }\end{array}$ & $\begin{array}{c}\text { Area } \\
\frac{8}{8}\end{array}$ \\
\hline & & & ------ & $-----\cdots$ & ---------- & \\
\hline 10 & 8.135 & VV & 0.1177 & 9.34445 & 1.05098 & 0.3591 \\
\hline 11 & 8.440 & VB & 0.2343 & 21.70663 & 1.15981 & 0.8343 \\
\hline 12 & 9.923 & $\mathrm{BBA}$ & 0.1389 & 24.28207 & 2.91463 & 0.9333 \\
\hline Total & : & & & 2601.86361 & 737.94321 & \\
\hline
\end{tabular}

Signal 3: MWD1 C, Sig=210,8 Ref=off

\begin{tabular}{|c|c|c|c|c|c|c|}
\hline $\begin{array}{c}\text { Peak } \\
\#\end{array}$ & $\begin{array}{c}\text { RetTime } \\
\text { [min] }\end{array}$ & Type & $\begin{array}{l}\text { Width } \\
\text { [min] }\end{array}$ & $\begin{array}{c}\text { Area } \\
{\left[\mathrm{mAU}^{*} s\right]}\end{array}$ & $\begin{array}{l}\text { Height } \\
{[\mathrm{mAU}]}\end{array}$ & $\begin{array}{c}\text { Area } \\
\frac{g}{8}\end{array}$ \\
\hline & 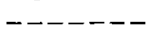 & & ------ & ---------- & ---------- & $-\cdots---$ \\
\hline 1 & 5.362 & $B B$ & 0.1218 & 43.44529 & 4.70236 & 0.7751 \\
\hline 2 & 5.895 & $\mathrm{BB}$ & 0.3202 & 133.78572 & 5.06465 & 2.3869 \\
\hline 3 & 7.512 & BP & 0.0560 & 5369.51807 & 1492.94690 & 95.8002 \\
\hline 4 & 7.841 & VV & 0.0507 & 17.66483 & 5.32040 & 0.3152 \\
\hline 5 & 7.928 & $\mathrm{VB}$ & 0.0524 & 40.49932 & 12.31502 & 0.7226 \\
\hline
\end{tabular}

Totals :

5604.913221520 .34932

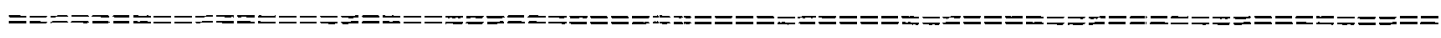

$\star \star \star$ End of Report $\star \star \star$ 


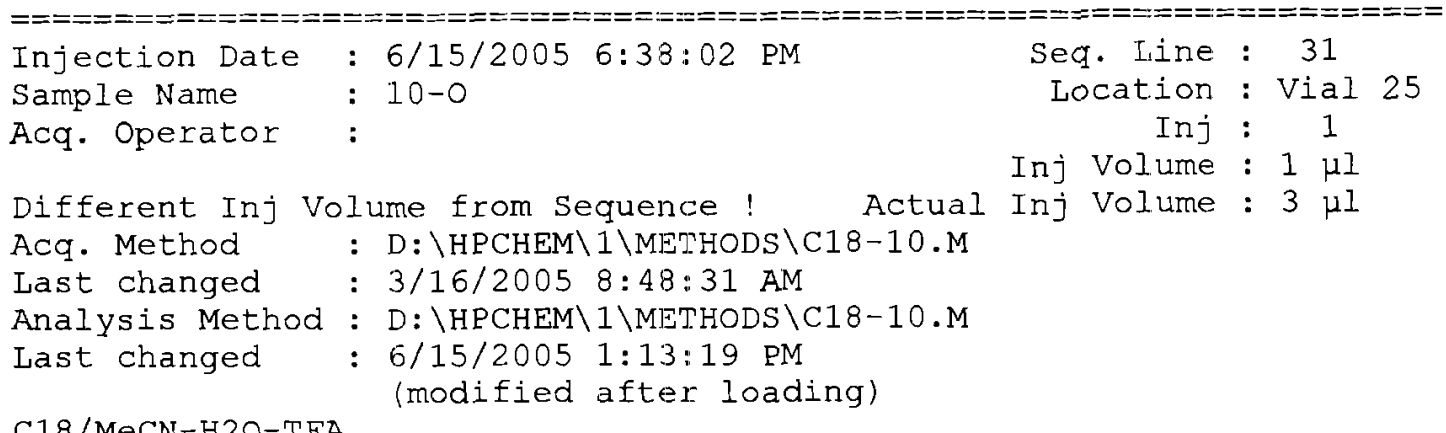

$\mathrm{C} 18 / \mathrm{MeCN}-\mathrm{H} 2 \mathrm{O}-\mathrm{TFA}$

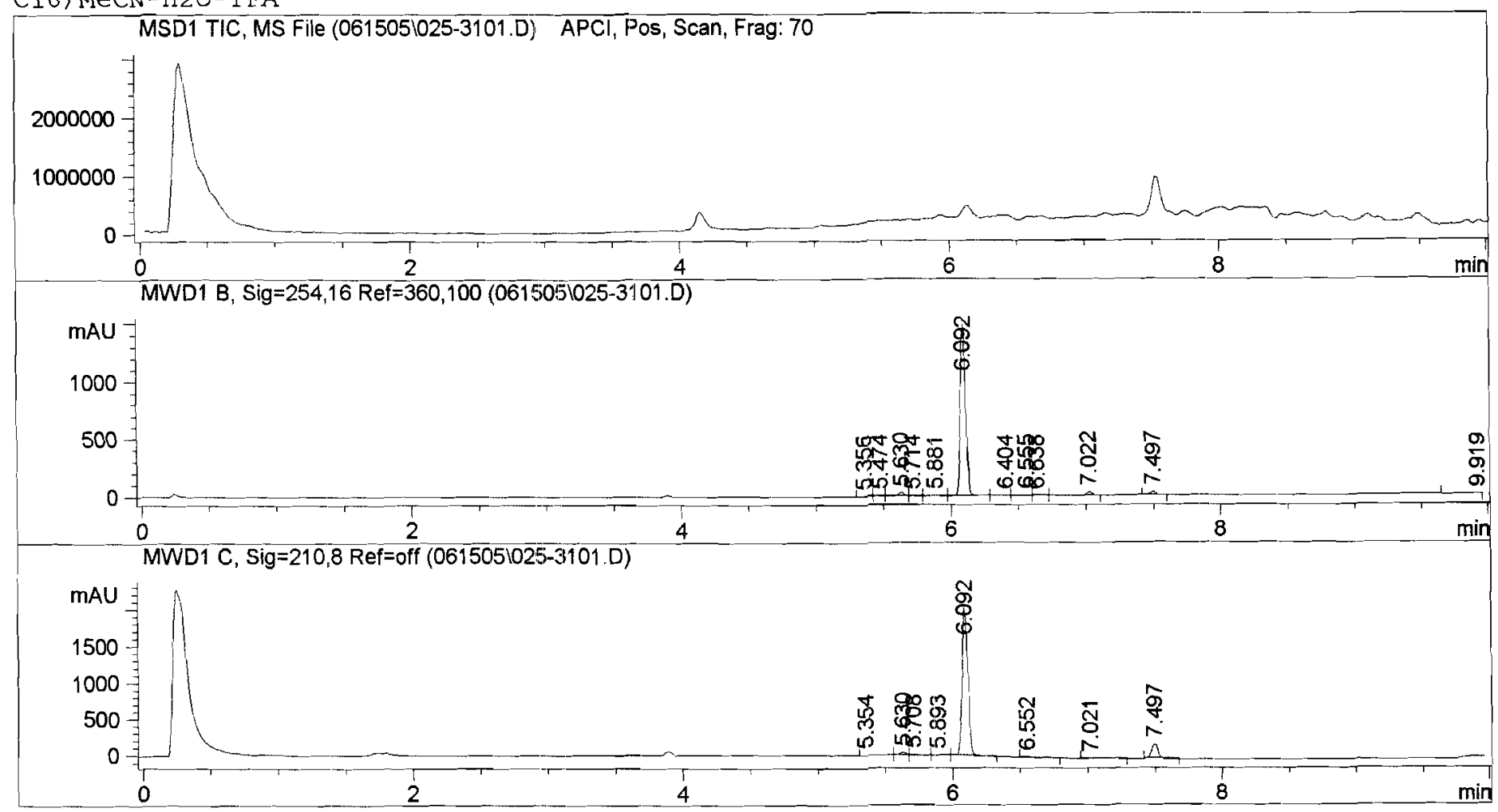

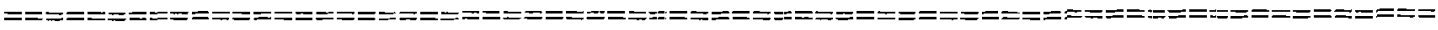

Area Percent Report

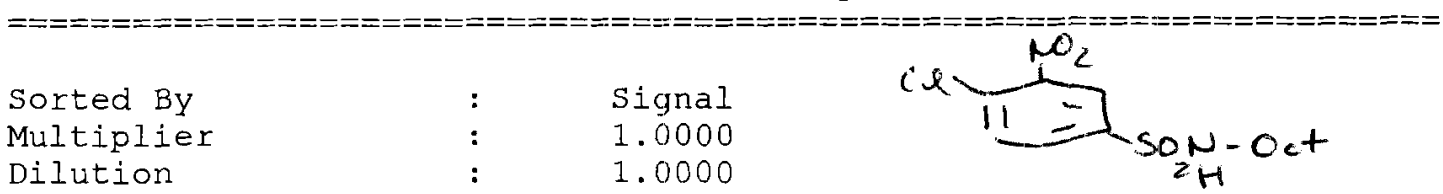

Signal 1: MSD1 TIC, MS File

Signal 2: MWD1 B, Sig=254,16 Ref=360, 100

\begin{tabular}{|c|c|c|c|c|c|c|}
\hline $\begin{array}{c}\text { Peak } \\
\#\end{array}$ & $\begin{array}{l}\text { RetTime } \\
\text { [min] }\end{array}$ & Type & $\begin{array}{l}\text { Width } \\
\text { [min] }\end{array}$ & $\begin{array}{c}\text { Area } \\
{\left[\mathrm{mAU}^{*} \mathrm{~s}\right]}\end{array}$ & $\begin{array}{l}\text { Height } \\
\text { [mAU] }\end{array}$ & $\begin{array}{c}\text { Area } \\
8\end{array}$ \\
\hline & & & & $--\cdots$ & & \\
\hline 1 & 5.356 & $\mathrm{PP}$ & 0.0495 & 4.46159 & 1.38641 & 0.1017 \\
\hline 2 & 5.474 & VV & 0.0410 & 2.65189 & 1.06593 & 0.0604 \\
\hline 3 & 5.630 & VV & 0.0446 & 78.43552 & 28.05469 & 1.7878 \\
\hline 4 & 5.714 & VV & 0.0496 & 7.67218 & 2.37799 & 0.1749 \\
\hline 5 & 5.881 & $\mathrm{VP}$ & 0.0654 & 9.43065 & 2.42232 & 0.2150 \\
\hline 6 & 6.092 & VV & 0.0440 & 4065.13892 & 1480.32544 & 92.6561 \\
\hline 7 & 6.404 & VV & 0.0986 & 6.24524 & $8.41334 \mathrm{e}-1$ & 0.1423 \\
\hline 8 & 6.555 & VV & 0.0530 & 17.05201 & 4.85172 & 0.3887 \\
\hline 9 & 6.638 & VV & 0.0779 & 6.60591 & $\begin{array}{c}1.16958 \\
\text { Page } 79 \text { of } 12\end{array}$ & 0.1506 \\
\hline
\end{tabular}

Instrument $16 / 16 / 20059: 48: 44 \mathrm{AM}$
$2: 1 \mathrm{FC}-72 \mathrm{HFE}-7100 / 5 \% \mathrm{KO}$ in DUR organic layor Table 2, Eutry 1 
Data File D: \HPCHEM \1\DATA $\backslash 061505 \backslash 025-3101 . \mathrm{D}$

\begin{tabular}{|c|c|c|c|c|c|c|}
\hline $\begin{array}{c}\text { Peak } \\
\#\end{array}$ & $\begin{array}{c}\text { RetTime } \\
\text { [min] }\end{array}$ & Type & $\begin{array}{l}\text { Width } \\
\text { [min] }\end{array}$ & $\begin{array}{c}\text { Area } \\
{\left[\mathrm{mAU}^{\star} \mathrm{s}\right]}\end{array}$ & $\begin{array}{l}\text { Height } \\
\text { [mAU] }\end{array}$ & $\begin{array}{c}\text { Area } \\
\frac{8}{\delta}\end{array}$ \\
\hline-- & ------- & & ------ & $----\ldots---$ & -------- & $----\cdots$ \\
\hline 10 & 7.022 & VB & 0.0494 & 86.91508 & 27.08982 & 1.9810 \\
\hline 11 & 7.497 & $\mathrm{BB}$ & 0.0493 & 76.10778 & 23.82836 & 1.7347 \\
\hline 12 & 9.919 & $\mathrm{BBA}$ & 0.1352 & 26.62268 & 3.00823 & 0.6068 \\
\hline
\end{tabular}

Totals :

$4387.33944 \quad 1576.42182$

Signal 3: MWD1 C, Sig=210,8 Ref:=off

\begin{tabular}{|c|c|c|c|c|c|c|}
\hline $\begin{array}{c}\text { Peak } \\
\#\end{array}$ & $\begin{array}{c}\text { RetTime } \\
\text { [min] }\end{array}$ & Type & $\begin{array}{c}\text { Width } \\
\text { [min] }\end{array}$ & $\begin{array}{c}\text { Area } \\
{\left[\mathrm{mAU}^{*} \mathrm{~s}\right]}\end{array}$ & $\begin{array}{l}\text { Height } \\
\text { [mAU] }\end{array}$ & $\begin{array}{c}\text { Area } \\
\quad \frac{8}{b}\end{array}$ \\
\hline & & & & & & \\
\hline 1 & 5.354 & BV & 0.1455 & 74.54178 & 6.51607 & 1.0370 \\
\hline 2 & 5.630 & VV & 0.0507 & 135.06009 & 40.67102 & 1.8790 \\
\hline 3 & 5.708 & VV & 0.0876 & 84.53787 & 13.05030 & 1.1761 \\
\hline 4 & 5.893 & VV & 0.0827 & 72.25646 & 11.91745 & 1.0052 \\
\hline 5 & 6.092 & VB & 0.0494 & 6134.75830 & 2022.06384 & 85.3475 \\
\hline 6 & 6.552 & $\mathrm{~PB}$ & 0.0372 & 13.55925 & 6.28872 & 0.1886 \\
\hline 7 & 7.021 & $\mathrm{BB}$ & 0.0328 & 16.74676 & 9.40405 & 0.2330 \\
\hline 8 & 7.497 & $\mathrm{BP}$ & 0.0495 & 656.51477 & 204.16776 & 9.1335 \\
\hline
\end{tabular}

Totals :

$7187.97528 \quad 2314.07921$ 

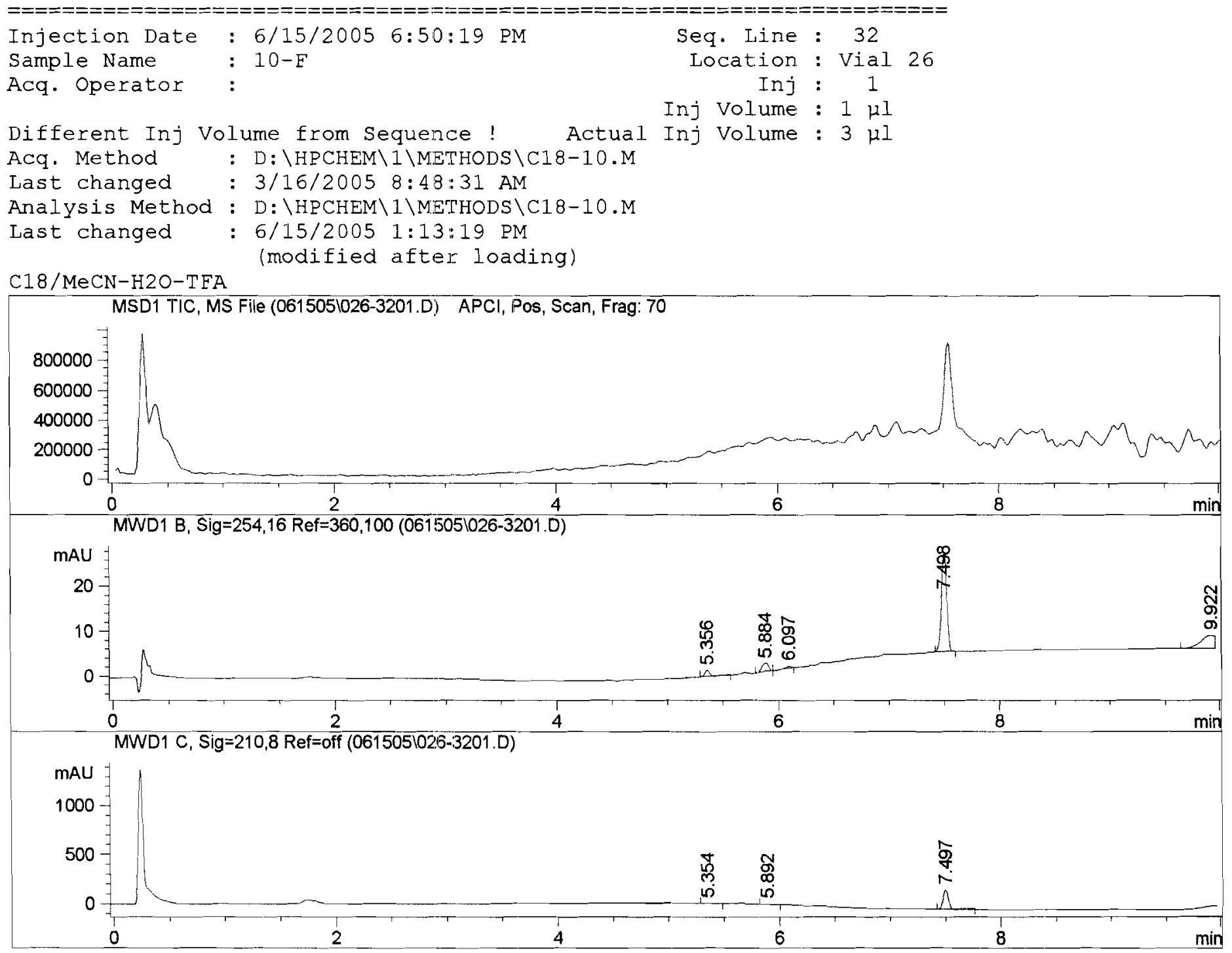

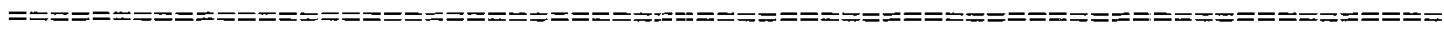

Area Percent Report

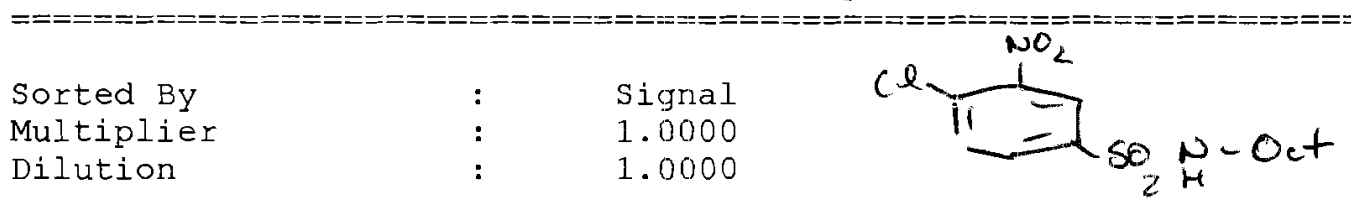

Signal 1: MSDI TIC, MS File
Seq. Line : 32

Inj : 1

Inj Volume: : $1 \mu l$

Inj Volume : $3 \mu \mathrm{l}$
Location : Vial 26
Signal 2: MWD1 B, Sig=254,16 Ref=360,100

\begin{tabular}{|c|c|c|c|c|c|c|}
\hline $\begin{array}{c}\text { Peak } \\
\#\end{array}$ & $\begin{array}{l}\text { RetTime } \\
\text { [min] }\end{array}$ & Type & $\begin{array}{l}\text { Width } \\
\text { [min] }\end{array}$ & $\begin{array}{c}\text { Area } \\
{[\mathrm{mAU} * \mathrm{~s}]}\end{array}$ & $\begin{array}{l}\text { Height } \\
\text { [MAU] }\end{array}$ & $\begin{array}{c}\text { Area } \\
8\end{array}$ \\
\hline 1 & 5.356 & $\mathrm{PP}$ & 0.0512 & 4.50740 & 1.41363 & 3.9 \\
\hline . & & $\mathrm{PP}$ & 0.0703 & 3961 & 1.9 & 78 \\
\hline 3 & 6.097 & VP & & & 5.050 & 1.43 \\
\hline 4 & 7.498 & $\mathrm{BB}$ & 0.0507 & 74.53676 & 22.49167 & 64.6305 \\
\hline 5 & 9.922 & $\mathrm{BBA}$ & 0.1385 & 26.63417 & 2.97285 & 23.0944 \\
\hline
\end{tabular}

Totals :

115.32761 
Signal 3: MWD1 C, Sig=210,8 Ref=off

\begin{tabular}{|c|c|c|c|c|c|c|}
\hline $\begin{array}{c}\text { Peak } \\
\#\end{array}$ & $\begin{array}{c}\text { RetTime } \\
{[\min ]}\end{array}$ & Type & $\begin{array}{l}\text { Width } \\
\text { [min] }\end{array}$ & $\begin{array}{c}\text { Area } \\
{\left[\mathrm{mAU}^{*} \mathrm{~s}\right]}\end{array}$ & $\begin{array}{l}\text { Height } \\
{[\mathrm{mAU}]}\end{array}$ & $\begin{array}{c}\text { Area } \\
\frac{8}{8}\end{array}$ \\
\hline- & -2 & & ------- & ---------1 & $-----\infty$ & \\
\hline 1 & 5.354 & $\mathrm{BB}$ & 0.1227 & 40.58451 & 4.27 & 892 \\
\hline 2 & 5.892 & BP & 0.3500 & 134.49353 & 4.66991 & 16.5337 \\
\hline 3 & 7.497 & $\mathrm{BB}$ & 0.0524 & 638.37201 & 193.70090 & 78.4771 \\
\hline 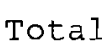 & & & & 813.45005 & 202.64951 & \\
\hline
\end{tabular}

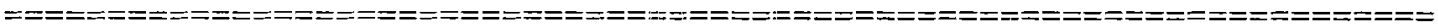
$\star \star \star$ Eind of Report $\star \star \star$ 


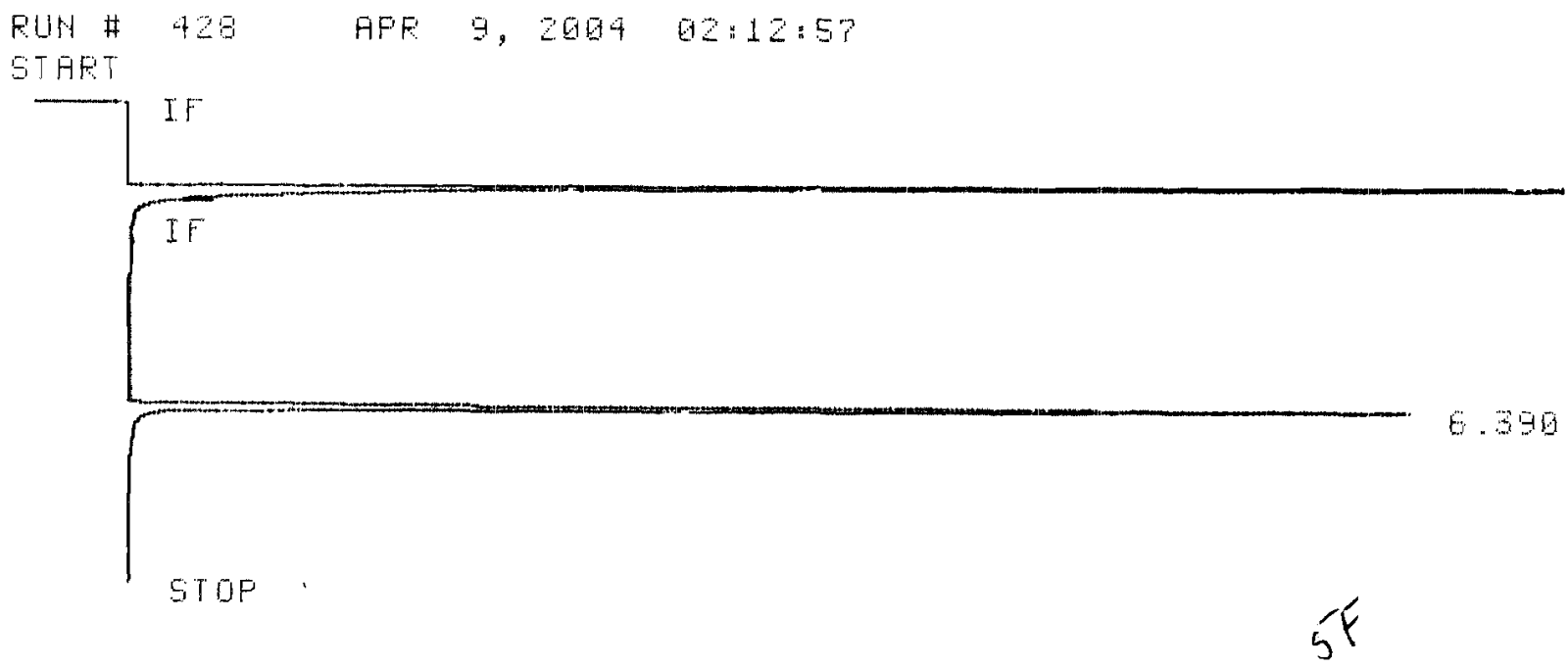

FutH 428

$$
\begin{array}{r}
\text { APF } 9,2004 \text { QZ:12:57 } \\
\text { SAMPLE\# } 9
\end{array}
$$

AREA

$\begin{array}{rrrrr}\text { PT } & \text { AREA TYFE } & \text { WIDTH } & \text { AREA\% } \\ \text { E.390 } & 123205 & \text { FE } & .073 & 100.00000\end{array}$

TOTFL AREA = 1232ES

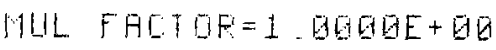

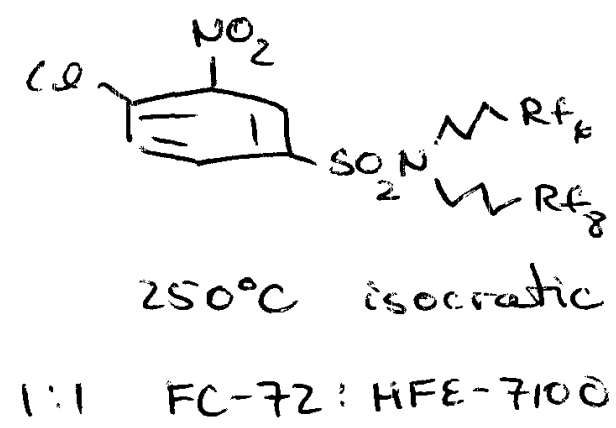

Table 2, Eutry 12 

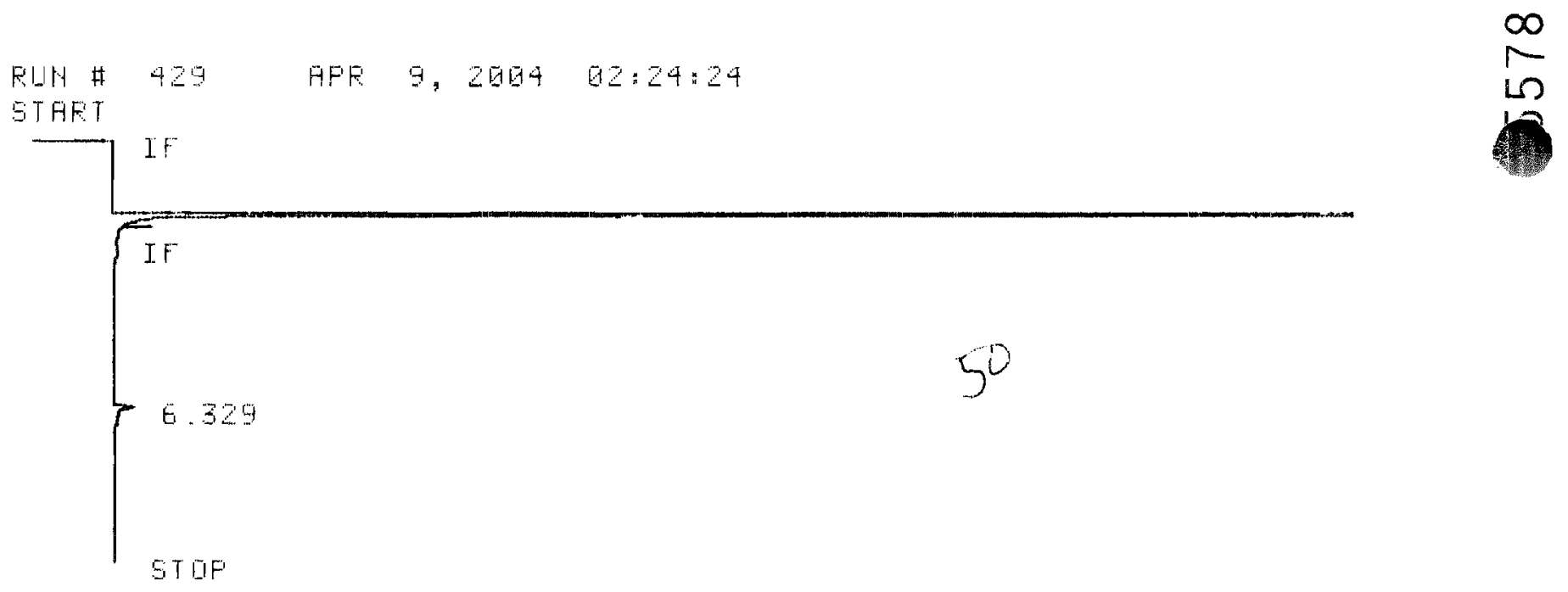

RLN\# 429

$$
\begin{array}{r}
\text { FPF } 9,2004 \quad 02: 24: 24 \\
\text { SFMFLE\# } 10
\end{array}
$$

AREF\%

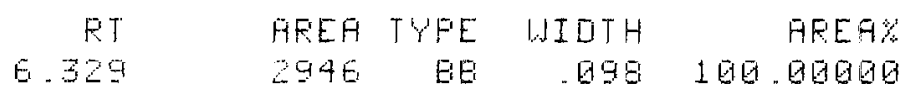

TOTAL AREA $=2946$

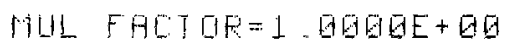

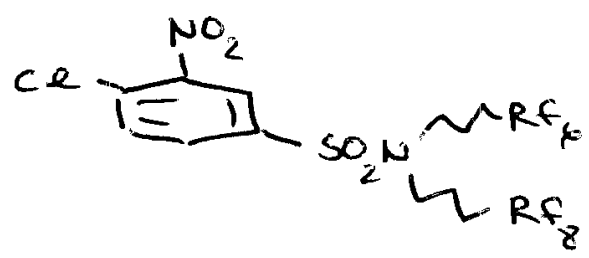

$250^{\circ} \mathrm{C}$ isocratic

$5 \% H_{2}$ in DMF

Table 2, Eutry 12

Page 84 of 126 
\begin{tabular}{l} 
RUHA \# 43E AFE 9, 2004 03:44:15 \\
START \\
\hline IF
\end{tabular}

I. $F$

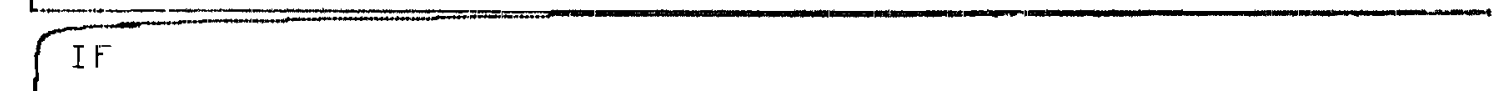

$m y 04-072$

Eutry 9 fluorous

RUNH 436

HO FUH FEAKS STOREQ

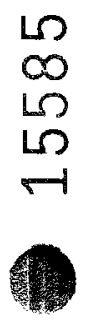

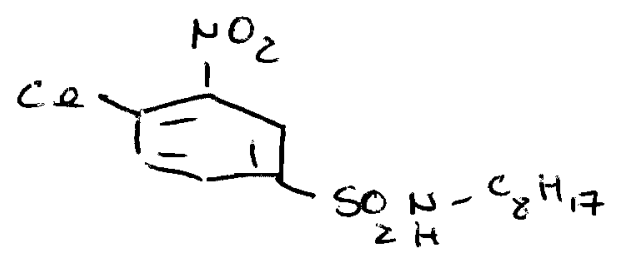

$$
\text { FC-72: HFE }-7100
$$

Table 2, Entry 13

Page 85 of 126 


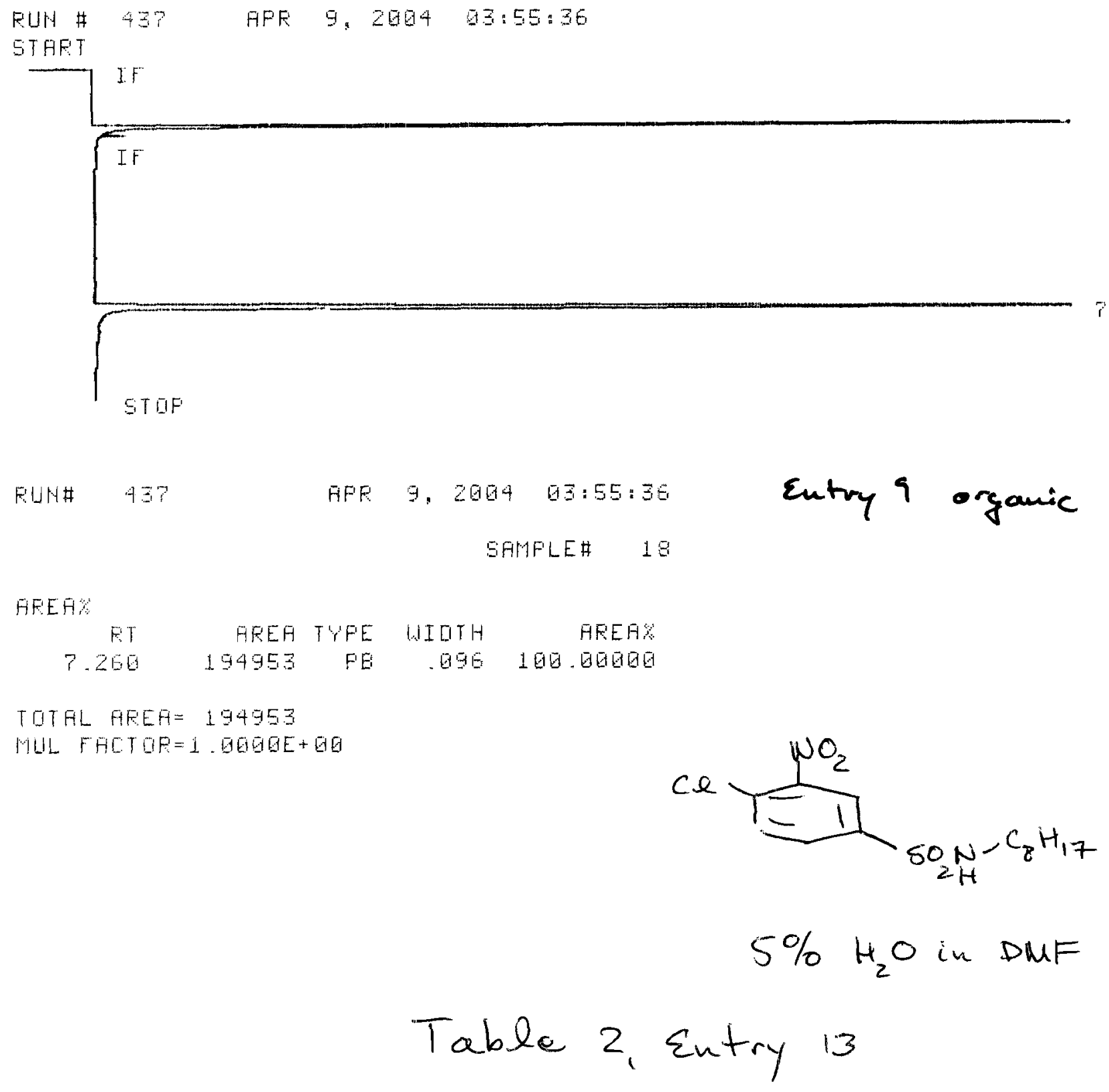




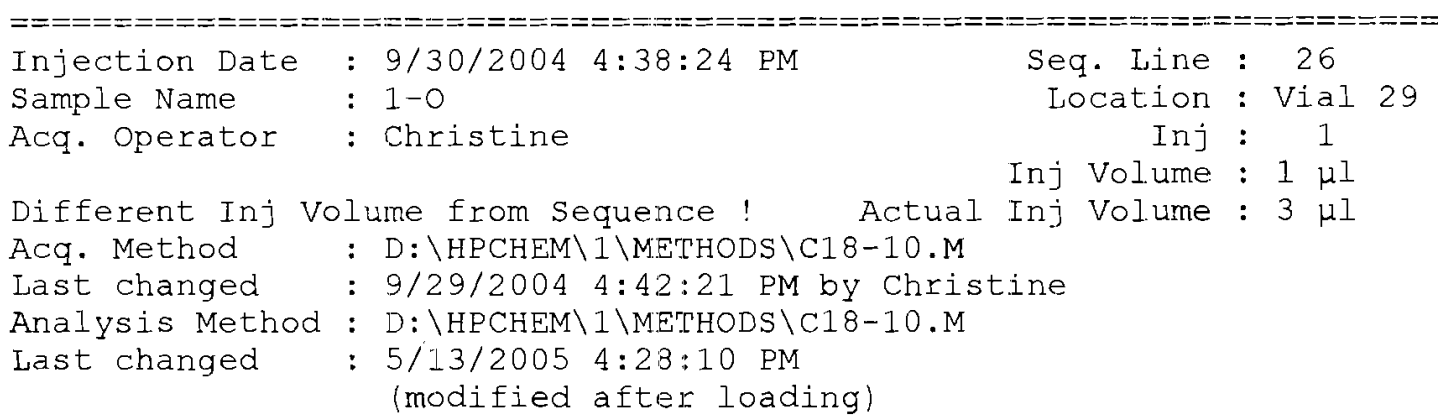

$\mathrm{C} 18 / \mathrm{MeCN}-\mathrm{H} 2 \mathrm{O}-\mathrm{TFA}$

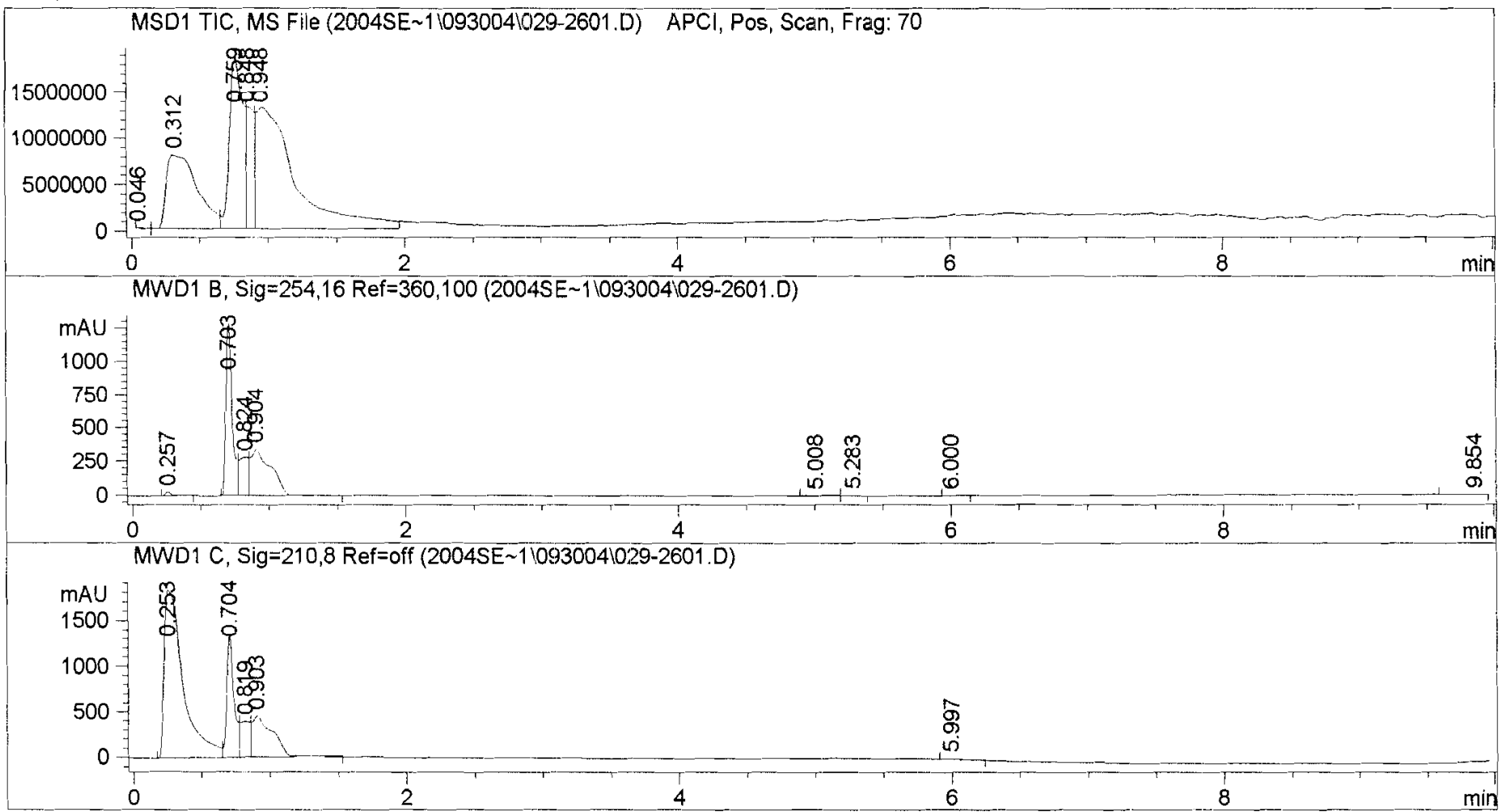

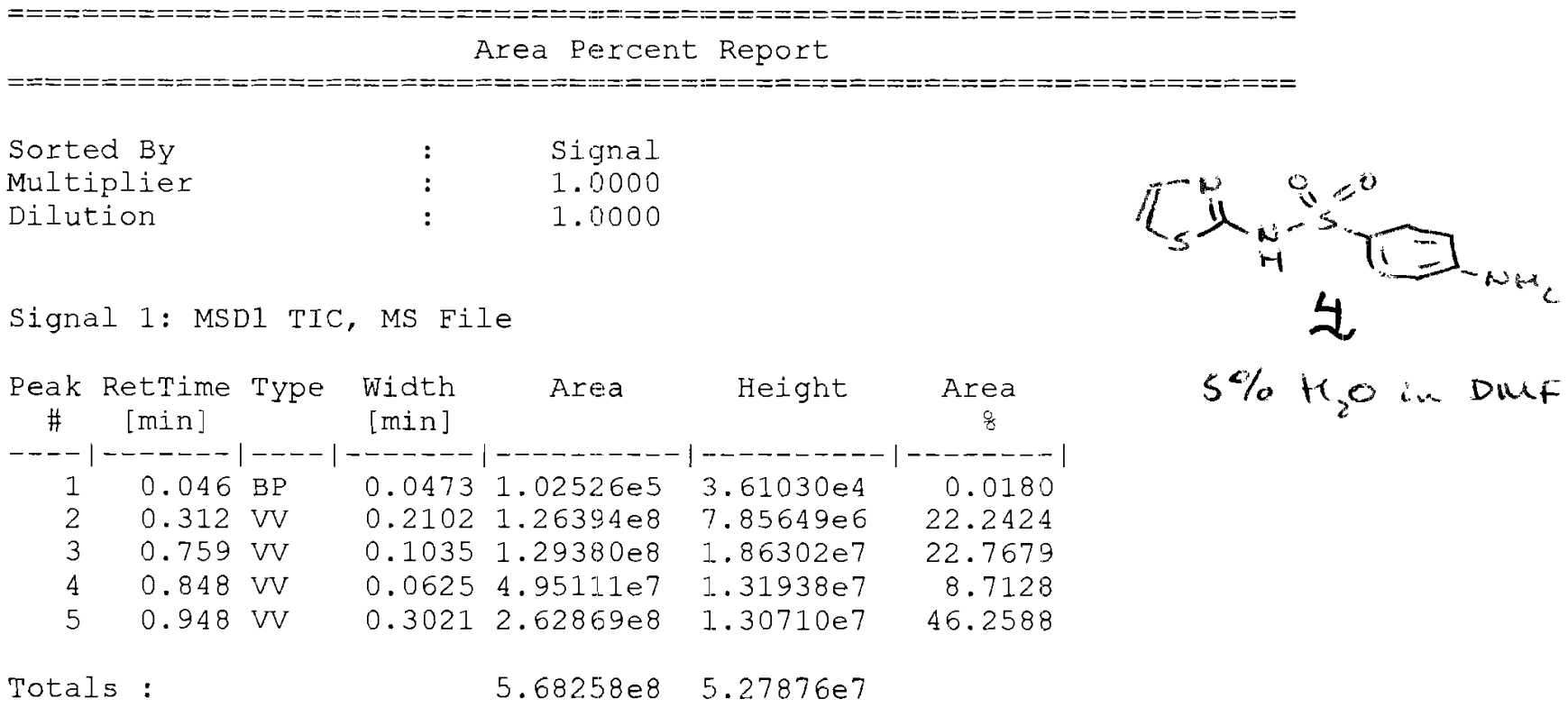




\begin{tabular}{|c|c|c|c|c|c|c|}
\hline $\begin{array}{c}\text { Peak } \\
\#\end{array}$ & $\begin{array}{l}\text { RetTime } \\
\text { [min] }\end{array}$ & Type & $\begin{array}{c}\text { Width } \\
\text { [min] }\end{array}$ & $\begin{array}{c}\text { Area } \\
{\left[\mathrm{mAU}{ }^{\star} \mathrm{s}\right]}\end{array}$ & $\begin{array}{l}\text { Height } \\
\text { [mAU] }\end{array}$ & $\begin{array}{c}\text { Area } \\
\& 5\end{array}$ \\
\hline 1 & 0.257 & $\mathrm{~PB}$ & 0.0610 & 98.51826 & 23.47231 & 1.0848 \\
\hline 2 & 0.703 & PV & 0.0495 & 4142.26270 & 1290.24170 & 45.6126 \\
\hline 3 & 0.824 & VV & 0.0697 & 1349.05713 & 282.43298 & 14.8552 \\
\hline 4 & 0.904 & VB & 0.1331 & 3451.85815 & 338.14822 & 38.0102 \\
\hline 5 & 5.008 & $\mathrm{PP}$ & 0.0999 & 8.13741 & 1.21816 & 0.0896 \\
\hline 6 & 5.283 & VB & 0.0798 & 3.31751 & $6.06262 e-1$ & 0.0365 \\
\hline 7 & 6.000 & $\mathrm{PP}$ & 0.0622 & 9.36692 & 2.26594 & 0.1031 \\
\hline 8 & 9.854 & $\mathrm{BBA}$ & 0.1744 & 18.87391 & 1.74221 & 0.2078 \\
\hline otal & : & & & 9081.39199 & 1940.12779 & \\
\hline
\end{tabular}

Signal 3: MWD1 C, Sig=210,8 Ref=off

\begin{tabular}{|c|c|c|c|c|c|c|}
\hline $\begin{array}{c}\text { Peak } \\
\#\end{array}$ & $\begin{array}{c}\text { RetTime } \\
\text { [min] }\end{array}$ & Type & $\begin{array}{l}\text { Width } \\
\text { [min] }\end{array}$ & $\begin{array}{c}\text { Area } \\
{\left[m A U^{*} s\right]}\end{array}$ & $\begin{array}{l}\text { Height } \\
\text { [mAU] }\end{array}$ & $\begin{array}{c}\text { Area } \\
\frac{9}{5}\end{array}$ \\
\hline & & & & & & \\
\hline 1 & 0.25 & PV & 0.145 & 1.7840 & 1827.4 & 60 \\
\hline 2 & 0.704 & VV & 0551 & 5012.05273 & 1358 & 16 \\
\hline 3 & 0.819 & VV & 0683 & 1838.51563 & 1766 & 189 \\
\hline 4 & 0.903 & $\mathrm{VB}$ & & 4804.82910 & 451.81287 & 16.2527 \\
\hline & 5.997 & $\mathrm{BB}$ & 0.0828 & 67.29945 & 11.40409 & 0.2276 \\
\hline
\end{tabular}

Totals :

$2.95633 e 4 \quad 4043.64558$

\section{$\star \star \star$ End of Report $\star \star \star$}




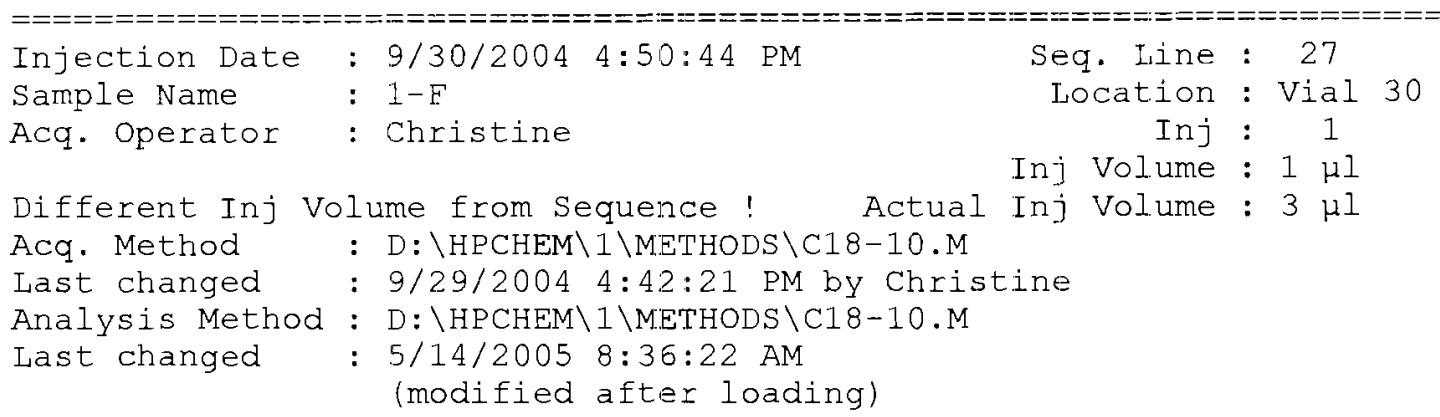

$\mathrm{C} 18 / \mathrm{MeCN}-\mathrm{H} 2 \mathrm{O}-\mathrm{TFA}$

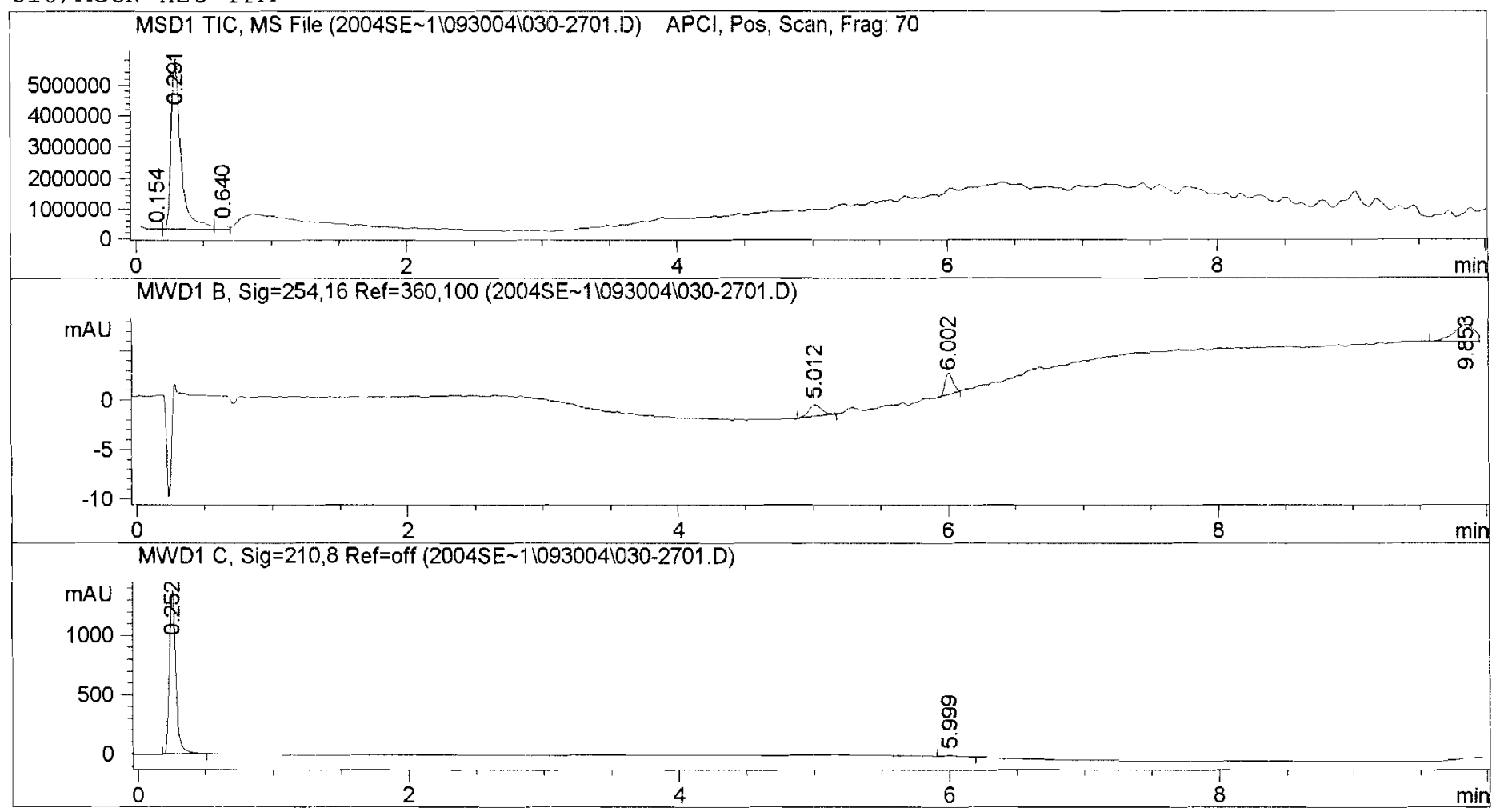

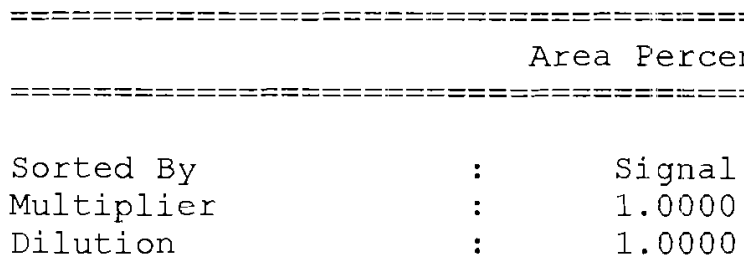

Signal 1: MSD1 TIC, MS File

\begin{tabular}{|c|c|c|c|c|c|c|}
\hline $\begin{array}{c}\text { Peak } \\
\#\end{array}$ & $\begin{array}{l}\text { RetTime } \\
\text { [min] }\end{array}$ & Type & $\begin{array}{l}\text { Width } \\
\text { [min] }\end{array}$ & Area & Height & $\begin{array}{c}\text { Area } \\
\frac{8}{8}\end{array}$ \\
\hline & & & & & & \\
\hline 1 & 0.154 & $\mathrm{BP}$ & 0.0451 & $4.45608 \mathrm{e} 4$ & $1.53612 \mathrm{e} 4$ & 0.151 .8 \\
\hline 2 & 0.291 & VV & 0.0766 & $2.83954 \mathrm{e} 7$ & $5.54692 e^{6}$ & 96.7387 \\
\hline 3 & 0.640 & VV & 0.1041 & $9.12706 \mathrm{e} 5$ & 1. $46113 e 5$ & 3.1094 \\
\hline
\end{tabular}

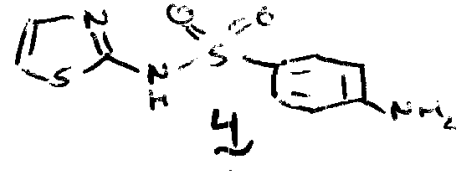

$F C=72 / H F E-7100$
Totals :
2.93527 e 7
$5.70840 e 6$ 
Signal 2: MWD1 B, Sig=254,16 Ref $=360,1.00$

\begin{tabular}{|c|c|c|c|c|c|c|}
\hline $\begin{array}{c}\text { Peak } \\
\#\end{array}$ & $\begin{array}{l}\text { RetTime } \\
\text { [min] }\end{array}$ & Type & $\begin{array}{c}\text { Width } \\
\text { [min] }\end{array}$ & $\begin{array}{c}\text { Area } \\
{\left[\mathrm{MAU}^{\star} \mathrm{s}\right]}\end{array}$ & $\begin{array}{l}\text { Height } \\
\text { [mAU] }\end{array}$ & $\begin{array}{c}\text { Area } \\
z\end{array}$ \\
\hline$=--$ & - & & & --------- & & ----2 \\
\hline 1 & 5.012 & $\mathrm{PP}$ & 0.1005 & 7.93322 & 1.17967 & 22.0864 \\
\hline 2 & 6.002 & $\mathrm{~PB}$ & 0.0644 & 9.33386 & 2.25020 & 25.9859 \\
\hline 3 & 9.853 & $\mathrm{BBA}$ & 0.1653 & 1.8 .65191 & 1.73678 & 51.9277 \\
\hline Total & : & & & 35.91898 & 5.16665 & \\
\hline
\end{tabular}

\section{Signal 3: MWD1 C, Sig=210,8 Ref=off}

\begin{tabular}{|c|c|c|c|c|c|c|}
\hline $\begin{array}{c}\text { Peak } \\
\#\end{array}$ & $\begin{array}{c}\text { RetTime } \\
\text { [min] }\end{array}$ & Type & $\begin{array}{l}\text { Width } \\
\text { [min] }\end{array}$ & $\begin{array}{c}\text { Area } \\
{\left[\mathrm{mAU}^{\star} \mathrm{S}\right]}\end{array}$ & $\begin{array}{l}\text { Height } \\
\text { [mAU] }\end{array}$ & $\begin{array}{c}\text { Area } \\
\qquad 8\end{array}$ \\
\hline 1 & 0252 & $P B$ & 0.0503 & 4559.07617 & 1387 & 6948 \\
\hline 2 & 5.999 & BB & 0.0783 & 60.29227 & 10.94611 & 1.3052 \\
\hline
\end{tabular}

Totals : $\quad 4619.36844 \quad 1398.32196$ 

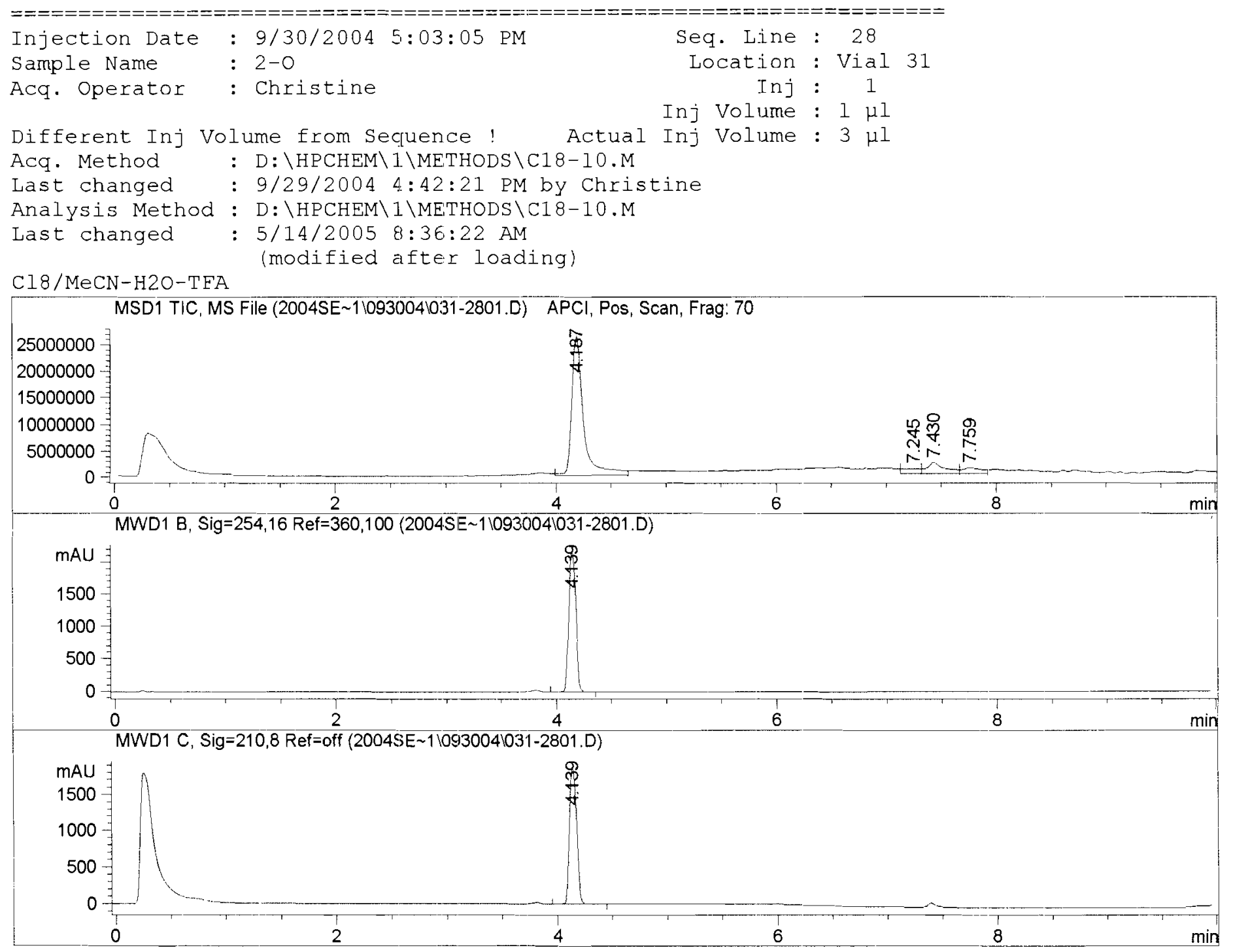

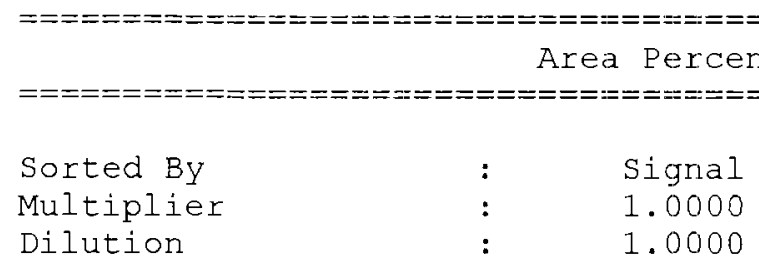

Signal 1: MSD1 TIC, MS File

\begin{tabular}{cccccc}
$\begin{array}{c}\text { Peak } \\
\text { \# }\end{array}$ & $\begin{array}{c}\text { RetTime Type } \\
\text { [min] }\end{array}$ & $\begin{array}{c}\text { Width } \\
{[\text { min] }}\end{array}$ & Area & Height & \multicolumn{1}{c}{$\begin{array}{c}\text { Area } \\
\text { \& }\end{array}$} \\
--1 & $-----1---1$ & & & \\
1 & $4.187 \mathrm{VV}$ & 0.1093 & $1.98090 \mathrm{e} 8$ & $2.66339 \mathrm{e} 7$ & 79.2400 \\
2 & $7.245 \mathrm{VV}$ & 0.1544 & $1.17989 \mathrm{e} 7$ & $1.04356 \mathrm{e}$ & 4.7198 \\
3 & $7.430 \mathrm{VV}$ & 0.1609 & $2.59312 \mathrm{e} 7$ & $2.18577 \mathrm{e} 6$ & 10.3730 \\
4 & $7.759 \mathrm{VV}$ & 0.1656 & $1.41672 \mathrm{e} 7$ & $1.11150 \mathrm{e}$ & 5.6672
\end{tabular}

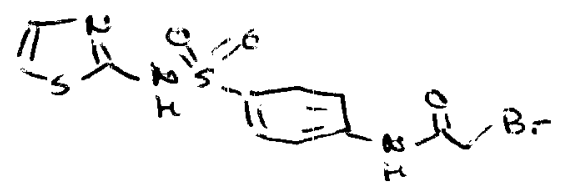

$5 \% \mathrm{H}_{2} \mathrm{O}$ : $\mathrm{W}$ DUiF

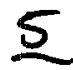

Totals :

$2.49987 \mathrm{e} 8 \quad 3.09748 \mathrm{e} 7$ 
Signal 2: MWD1 B, Sig=254,16 Ref=360,100

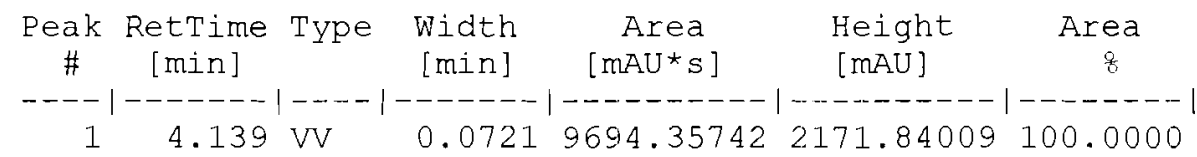

Totals : $9694.35742 \quad 2171.84009$

Signal 3: MWD1 C, Sig=210,8 Ref=off

\begin{tabular}{|c|c|c|c|c|c|c|}
\hline $\begin{array}{c}\text { Peak } \\
\#\end{array}$ & $\begin{array}{c}\text { RetTime } \\
\text { [min] }\end{array}$ & Type & $\begin{array}{l}\text { width } \\
\text { [min] }\end{array}$ & $\begin{array}{c}\text { Area } \\
{\left[\mathrm{m} A U^{\star} \mathrm{S}\right]}\end{array}$ & $\begin{array}{l}\text { Height } \\
\text { [mAU] }\end{array}$ & $\begin{array}{c}\text { Area } \\
\frac{8}{8}\end{array}$ \\
\hline- & ------- & & ------- & $--\cdots-------$ & ------------ & $-\ldots$ \\
\hline 1 & 4.139 & $\mathrm{PP}$ & 0.0754 & 8565.51270 & 1870.48608 & 100.0000 \\
\hline Total & : & & & 8565.51270 & .870 .48608 & \\
\hline
\end{tabular}

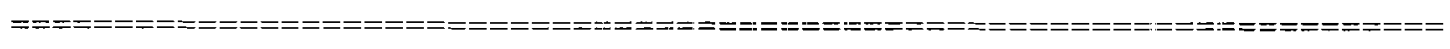
$\star \star \star$ End of Report $\star \star \star$ 


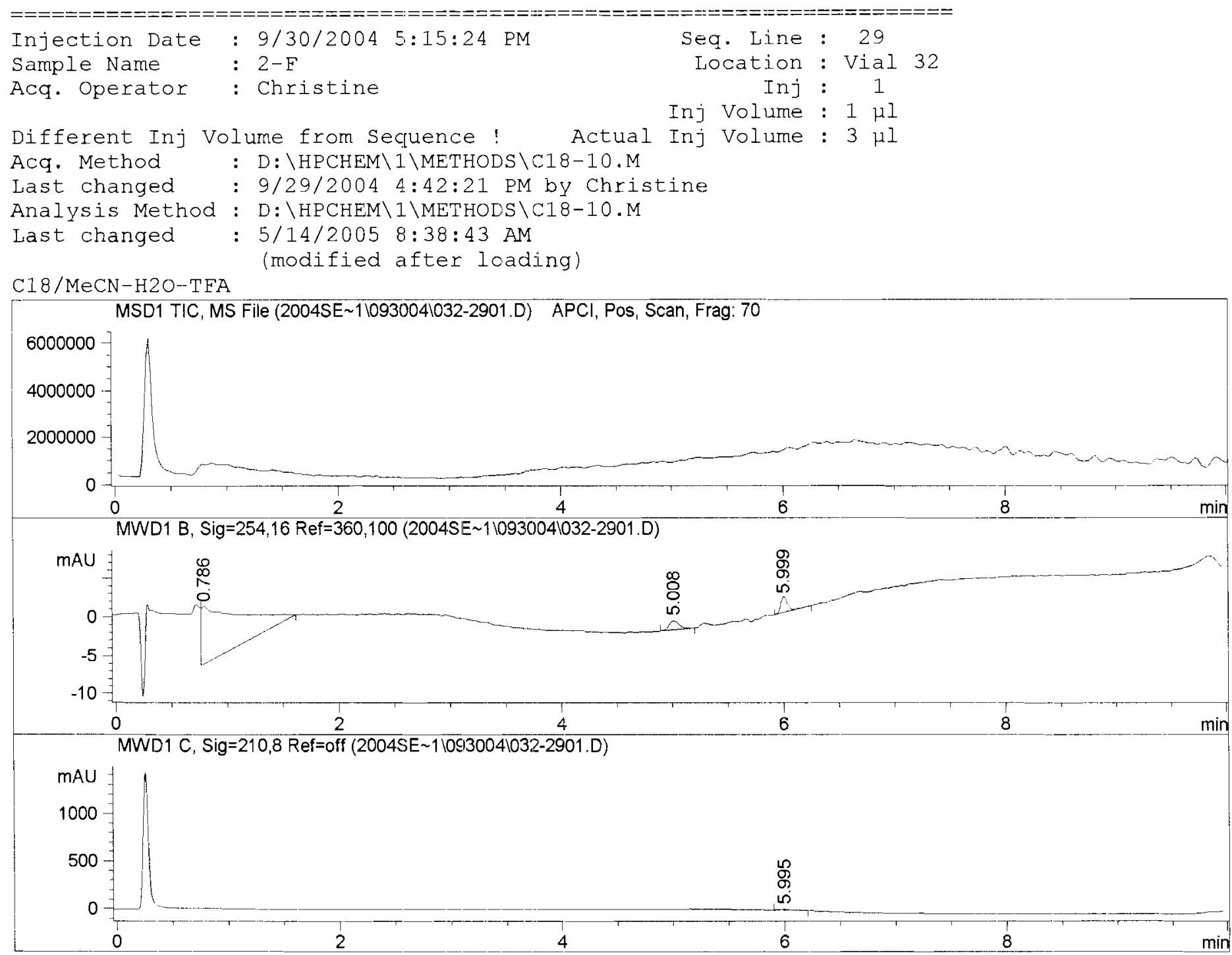

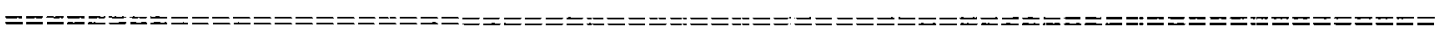

Area Percent Report

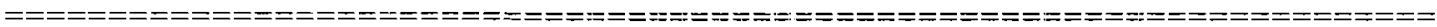

$\begin{array}{lll}\text { Sorted By } & : & \text { Signal } \\ \text { Multiplier } & : & 1.0000 \\ \text { Dilution } & : & 1.0000\end{array}$

Signal 1: MSD1 TIC, MS File

Signal 2: MWD1 B, Sig=254,16 Ref=360,100

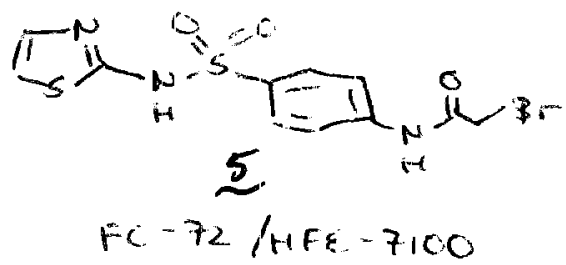

\begin{tabular}{|c|c|c|c|c|c|c|}
\hline $\begin{array}{c}\text { Peak } \\
\#\end{array}$ & $\begin{array}{l}\text { RetTime } \\
\text { [min] }\end{array}$ & Type & $\begin{array}{l}\text { Width } \\
\text { [min] }\end{array}$ & $\begin{array}{c}\text { Area } \\
{\left[\mathrm{mAU}{ }^{\star} \mathrm{s}\right]}\end{array}$ & $\begin{array}{l}\text { Height } \\
\text { [mAU] }\end{array}$ & $\begin{array}{c}\text { Area } \\
8\end{array}$ \\
\hline 1 & 0.786 & VB & 0.1028 & 55.02948 & 7.31351 & 76.9874 \\
\hline 2 & 5.008 & $\mathrm{PP}$ & 0.1049 & 7.83074 & 1.15734 & 10.9554 \\
\hline 3 & 5.999 & $\mathrm{PP}$ & 0.0640 & 8.61832 & 2.09595 & 12.0572 \\
\hline
\end{tabular}

Totals :

$71.47854 \quad 10.56681$ 
Signal 3: MWD1 C, Sig=210,8 Ref=off

\begin{tabular}{|c|c|c|c|c|c|c|}
\hline $\begin{array}{c}\text { Peak } \\
\#\end{array}$ & $\begin{array}{c}\text { RetTime } \\
\text { [min] }\end{array}$ & Type & $\begin{array}{l}\text { Width } \\
\text { [min] }\end{array}$ & $\begin{array}{c}\text { Area } \\
{\left[\mathrm{mAU}^{\star} \mathrm{s}\right]}\end{array}$ & $\begin{array}{l}\text { Height } \\
\text { [mAU] }\end{array}$ & $\begin{array}{c}\text { Area } \\
\text { के }\end{array}$ \\
\hline & ------ & & ------ & --------- & ------- & $--\cdots---\cdots$ \\
\hline 1 & 5.995 & $\mathrm{BB}$ & 0.0822 & 61.83353 & 10.88962 & 100.0000 \\
\hline Total & : & & & 61.83353 & 10.88962 & \\
\hline
\end{tabular}

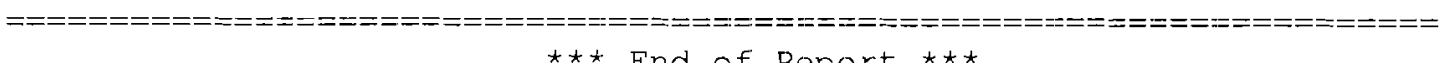




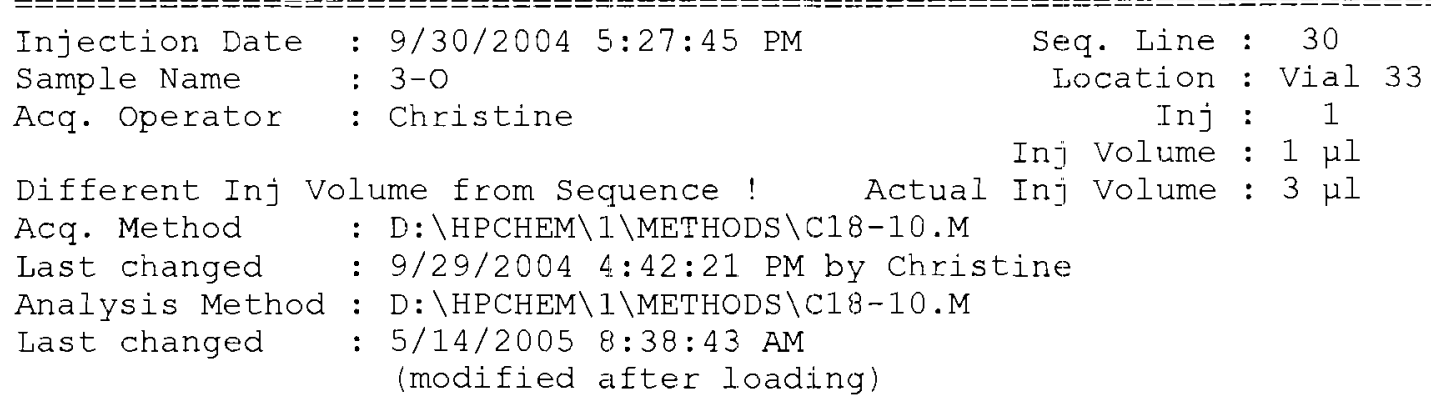

$\mathrm{C} 18 / \mathrm{MeCN}-\mathrm{H} 2 \mathrm{O}-\mathrm{TFA}$

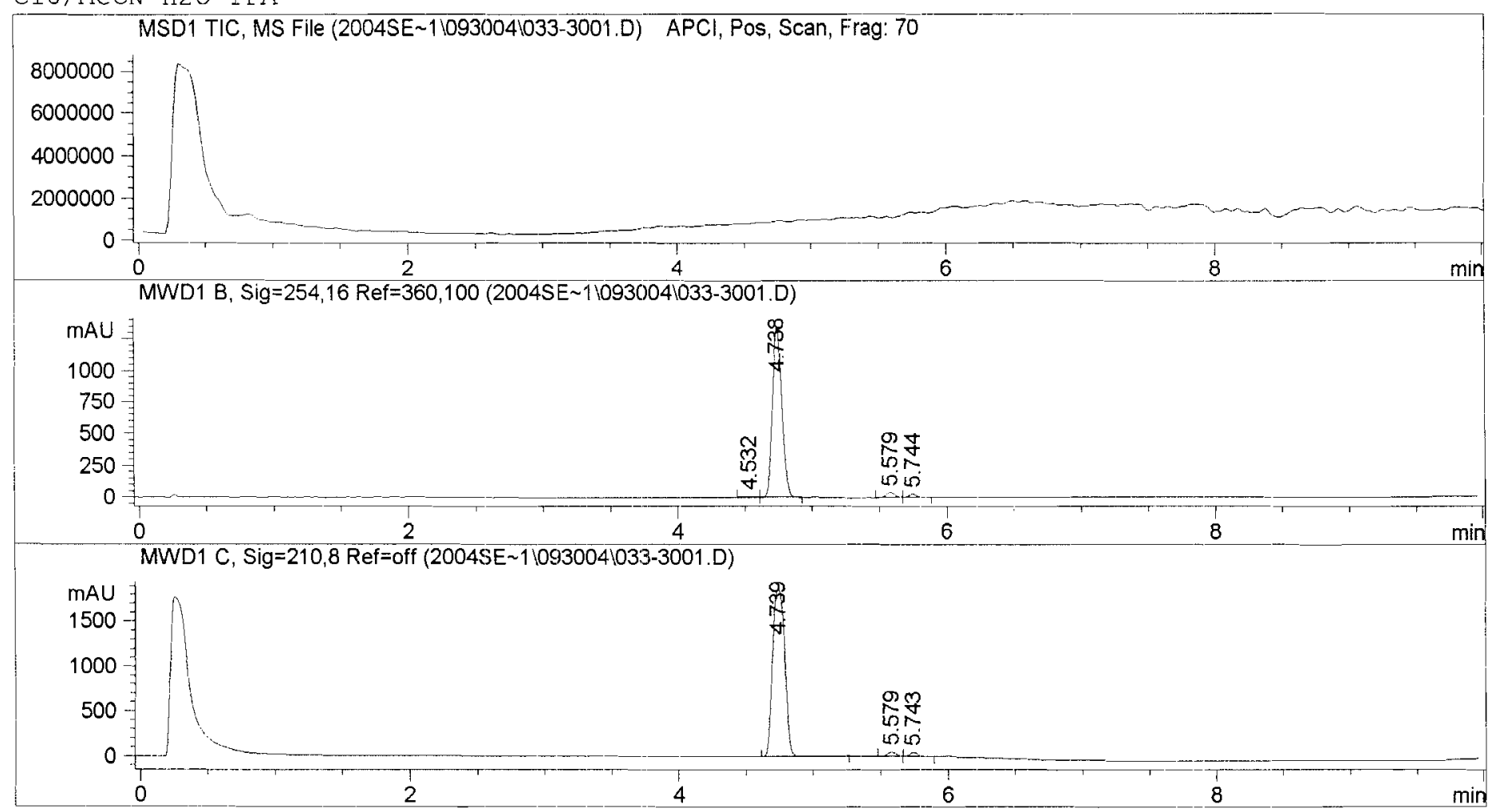

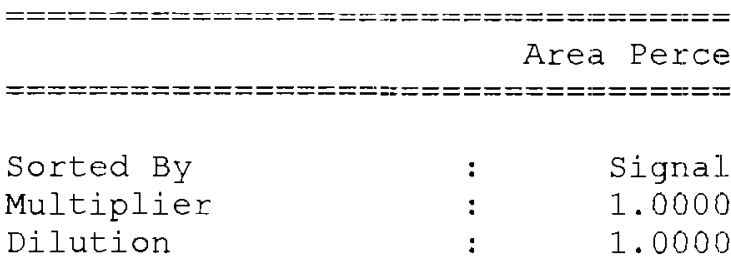

Signal 1: MSDl TIC, MS File

Signal 2: MWD1 B, Sig=254,16 Re $E=360,1.00$

\begin{tabular}{cccccc}
$\begin{array}{c}\text { Peak } \\
\text { \# }\end{array}$ & $\begin{array}{c}\text { RetTime Type } \\
\text { [min] }\end{array}$ & $\begin{array}{c}\text { Width } \\
\text { [min] }\end{array}$ & $\begin{array}{c}\text { Area } \\
{[\text { mAU*s] }}\end{array}$ & $\begin{array}{c}\text { Height } \\
\text { [mAU] }\end{array}$ & \multicolumn{1}{c}{$\begin{array}{c}\text { Area } \\
\text { E }\end{array}$} \\
\hline 1 & $4.532 \mathrm{VP}$ & 0.0864 & 3.10296 & $5.61790 \mathrm{e}-1$ & 0.0456 \\
2 & $4.738 \mathrm{VV}$ & 0.0762 & 6526.98047 & 1354.55603 & 95.9192 \\
3 & $5.579 \mathrm{VV}$ & 0.0687 & 174.23749 & 40.12130 & 2.5606 \\
4 & $5.744 \mathrm{VP}$ & 0.0647 & 100.34248 & 24.04543 & 1.4746
\end{tabular}

Totals :

$6804.65339 \quad 1419.28455$

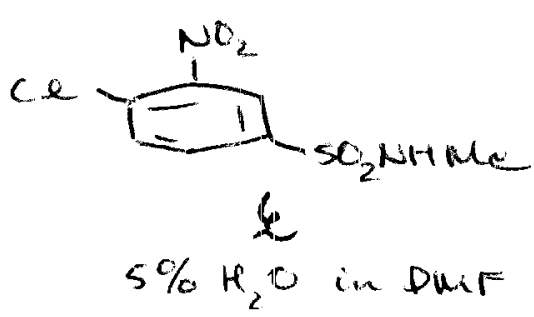


Signal 3: MWD1 C, Sig=210,8 Ref=off

\begin{tabular}{|c|c|c|c|c|c|c|}
\hline $\begin{array}{c}\text { Peak } \\
\#\end{array}$ & $\begin{array}{c}\text { RetTime } \\
\text { [min] }\end{array}$ & Type & $\begin{array}{l}\text { Width } \\
\text { [min] }\end{array}$ & $\begin{array}{c}\text { Area } \\
{\left[\mathrm{MAU}^{\star} \mathrm{s}\right]}\end{array}$ & $\begin{array}{l}\text { Height } \\
\text { [mAU] }\end{array}$ & $\begin{array}{c}\text { Area } \\
\text { 多 }\end{array}$ \\
\hline & & & - & ---------- & $-2--2--1$ & $-5+2$ \\
\hline 1 & 4.739 & $\mathrm{~PB}$ & 0.1059 & $1.17821 \mathrm{e} 4$ & $186 \mathrm{~L} .00574$ & 96.2984 \\
\hline 2 & 5.579 & BV & 0.0712 & 22.7 .73351 & 49.90382 & 1.8613 \\
\hline 3 & 5.743 & VV & 0.0715 & 225.15736 & 47.34162 & 1.8403 \\
\hline
\end{tabular}

Totals : $\quad 1.22350$ e $\quad 1958.25117$

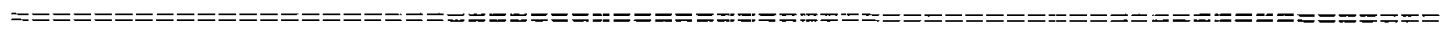

$\star \star \star$ End of Report $\star \star \star$ 


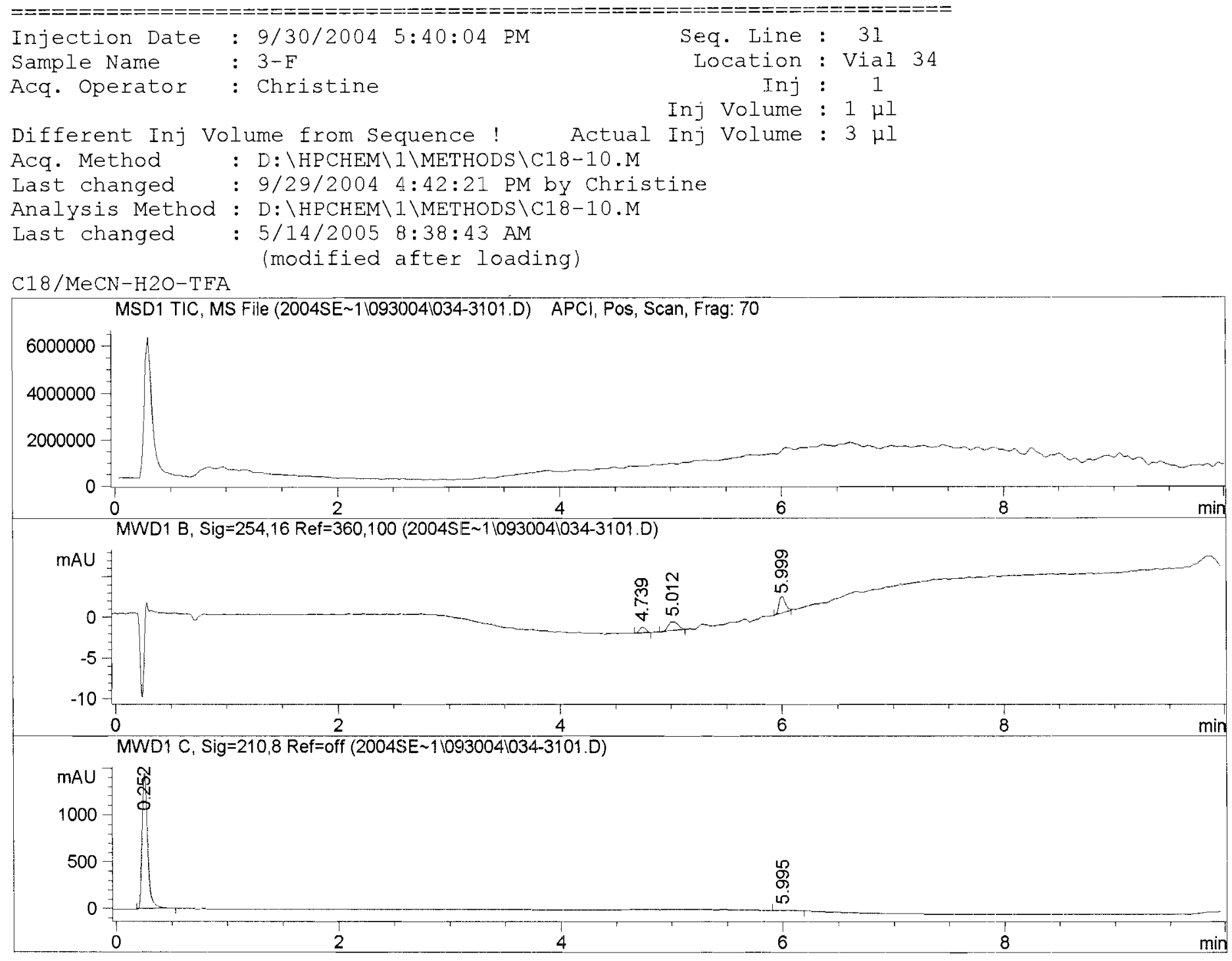

\begin{tabular}{|c|c|c|}
\hline \multirow{2}{*}{\multicolumn{3}{|c|}{$\begin{aligned} & \text { Area Perce } \\
&========:=\end{aligned}$}} \\
\hline & & \\
\hline Sorted By & : & Signa \\
\hline Multiplier & : & 1.0000 \\
\hline Dilution & : & 1.000 \\
\hline
\end{tabular}

Signal 1: MSD1 TIC, MS File

Signal 2: MWD1 B, Sig=254,16 Ref=360,100

\begin{tabular}{|c|c|c|c|c|c|c|}
\hline $\begin{array}{c}\text { Peak } \\
\#\end{array}$ & $\begin{array}{c}\text { RetTime } \\
\text { [min] }\end{array}$ & Type & $\begin{array}{l}\text { Width } \\
\text { [min] }\end{array}$ & $\begin{array}{c}\text { Area } \\
{\left[\mathrm{mAU}^{\star} \mathrm{s}\right]}\end{array}$ & $\begin{array}{l}\text { Height } \\
\text { [mAU] }\end{array}$ & $\begin{array}{c}\text { Area } \\
\frac{8}{8}\end{array}$ \\
\hline - & ------ & & & 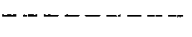 & ---------- & \\
\hline 1 & 4.739 & PP & 0.0641 & 2.93306 & $7.12031 e-1$ & 16.2400 \\
\hline 2 & 5.012 & $\mathrm{PP}$ & 0.1031 & 6.58086 & 1.08018 & 36.4374 \\
\hline 3 & 5.999 & PB & 0.0604 & 8.54683 & 2.14959 & 47.322 .7 \\
\hline
\end{tabular}

Totals :

$18.06076 \quad 3.94179$

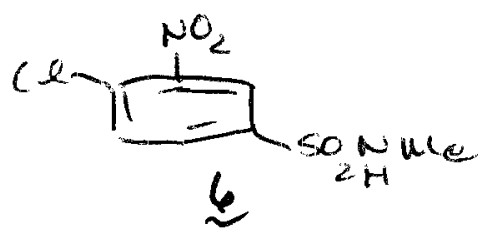

$F C-72 / H F E-7100$ 
Signal 3: MWD1 C, Sig=210,8 Ref=off

\begin{tabular}{|c|c|c|c|c|c|c|}
\hline $\begin{array}{c}\text { Peak } \\
\#\end{array}$ & $\begin{array}{c}\text { RetTime } \\
\text { [min] }\end{array}$ & Type & $\begin{array}{l}\text { Width } \\
\text { [min] }\end{array}$ & $\begin{array}{c}\text { Area } \\
{\left[m A{ }^{*} s\right]}\end{array}$ & $\begin{array}{l}\text { Height } \\
\text { [mAU] }\end{array}$ & $\begin{array}{c}\text { Area } \\
\quad 8\end{array}$ \\
\hline & -------- & & ------ & --------- & ---------- & ------- \\
\hline 1 & 0.252 & $\mathrm{~PB}$ & 0.0542 & 5038.49707 & 1461.03088 & 98.8988 \\
\hline 2 & 5.995 & $\mathrm{BB}$ & 0.0766 & 56.10065 & 10.46047 & L. 1012 \\
\hline Tota & : & & & 5094.59772 & 1471.49135 & \\
\hline
\end{tabular}

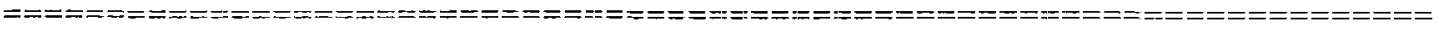
$\star \star \star$ End of Report $\star \star \star$ 


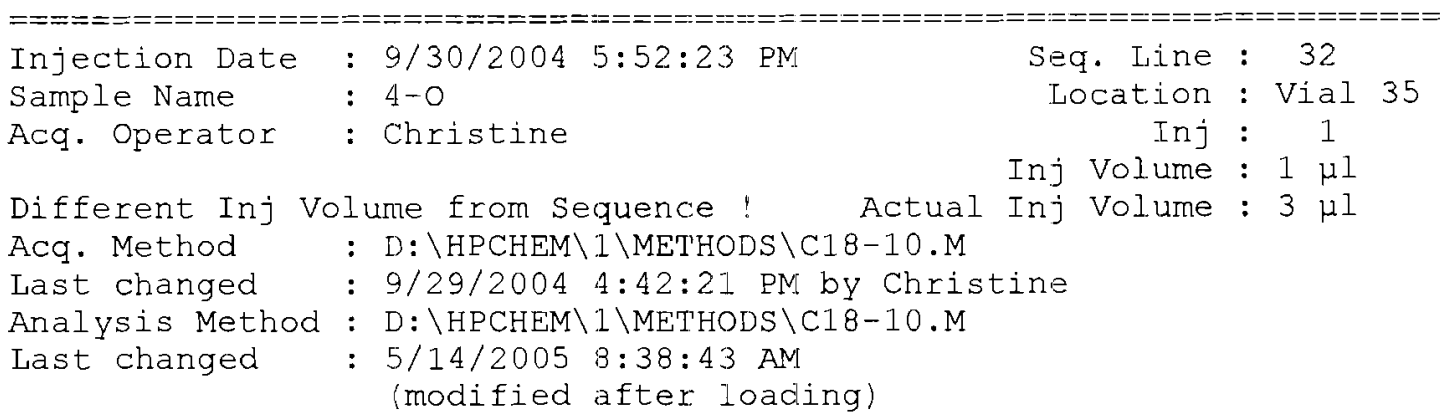

$\mathrm{C} 18 / \mathrm{MeCN}-\mathrm{H} 2 \mathrm{O}-\mathrm{TFA}$
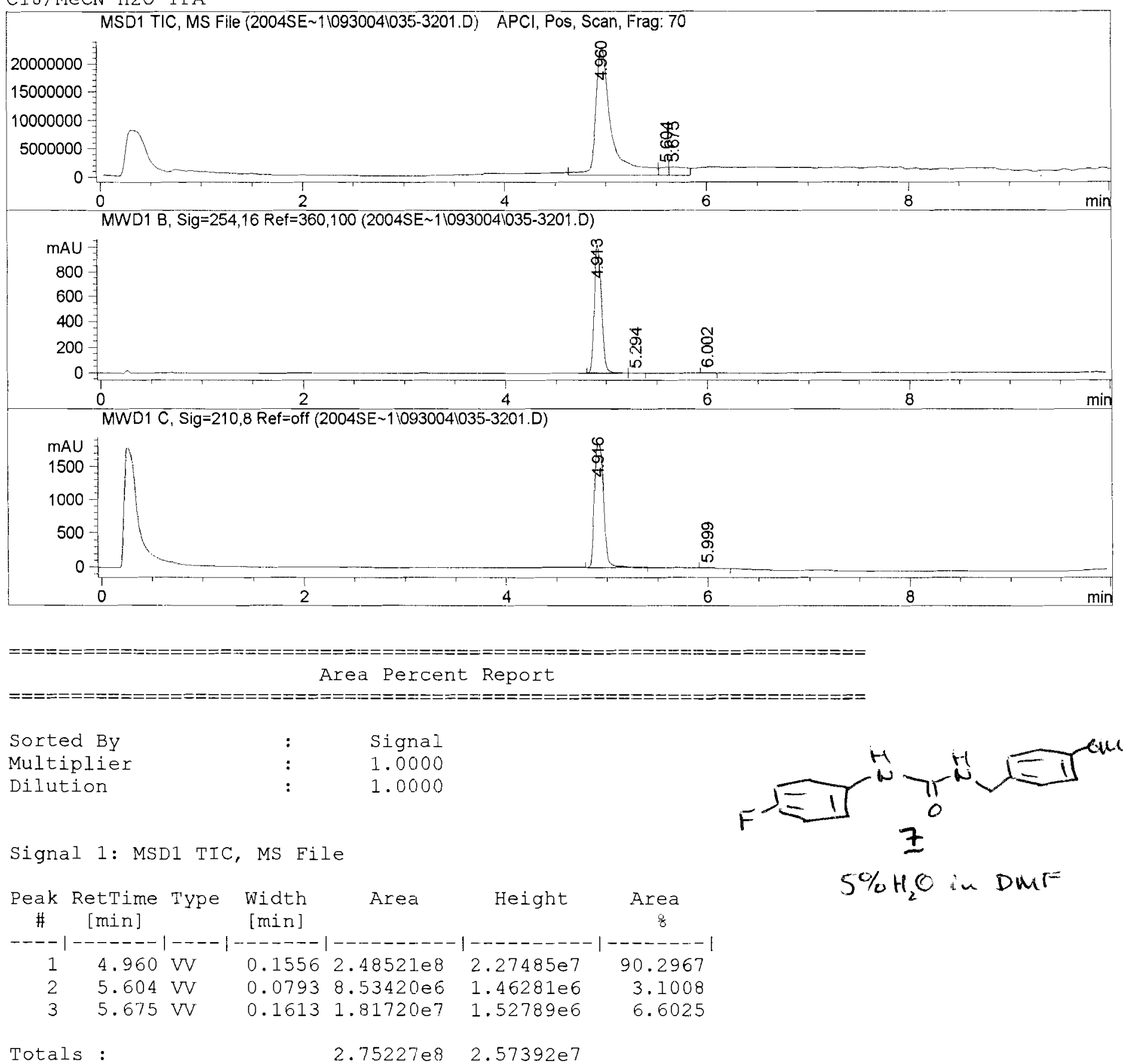

Sorted By Multiplier Dilution

Signal 1: MSD1 TIC, MS File

\begin{tabular}{|c|c|c|c|c|c|c|}
\hline $\begin{array}{c}\text { Peak } \\
\#\end{array}$ & $\begin{array}{c}\text { RetTime } \\
\text { [min] }\end{array}$ & Type & $\begin{array}{l}\text { Width } \\
\text { [min] }\end{array}$ & Area & Height & $\begin{array}{c}\text { Area } \\
\frac{g}{8}\end{array}$ \\
\hline & & & ------ & & & \\
\hline 1 & 4.960 & VV & 0.1556 & $2.48521 \mathrm{e} 8$ & $2.27485 \mathrm{e} 7$ & 90.2967 \\
\hline 2 & 5.604 & VV & 0.0793 & $8.53420 \mathrm{e} 6$ & $1.46281 \mathrm{e} 6$ & 3.1008 \\
\hline 3 & 5.675 & VV & 0.1613 & $1.81720 \mathrm{e} 7$ & $1.52789 \mathrm{e} 6$ & 6.6025 \\
\hline
\end{tabular}

Totals :

Signal

1.0000

1.0000

$5 \% \mathrm{H}_{2} \mathrm{O}$ in $\mathrm{DM}=$ 
Signal 2: MWD1 B, Sig=254,16 Ref=360,100

\begin{tabular}{|c|c|c|c|c|c|c|}
\hline $\begin{array}{c}\text { Peak } \\
\#\end{array}$ & $\begin{array}{c}\text { RetTime } \\
\text { [min] }\end{array}$ & Type & $\begin{array}{c}\text { Width } \\
{[\min ]}\end{array}$ & $\begin{array}{c}\text { Area } \\
{\left[\mathrm{mAU}^{*} \mathrm{~s}\right]}\end{array}$ & $\begin{array}{l}\text { Height } \\
\text { [mAU] }\end{array}$ & $\begin{array}{c}\text { Area } \\
8\end{array}$ \\
\hline & 2 & & ------ & $-------\cdots-$ & ---------- & $-\cdots----$ \\
\hline 1 & 4.913 & BV & 0.0769 & 4983.52148 & 1021.40460 & 99.6402 \\
\hline 2 & 5.294 & VV & 0.0955 & 9.76376 & 1.43243 & 0.1952 \\
\hline 3 & 6.002 & $\mathrm{~PB}$ & 0.0601 & 8.23131 & 2.08521 & 0.1646 \\
\hline
\end{tabular}

Totals :

$5001.51655 \quad 1024.92225$

Signal 3: MWD1 C, Sig=210,8 Ref=off

\begin{tabular}{|c|c|c|c|c|c|c|}
\hline $\begin{array}{c}\text { Peak } \\
\#\end{array}$ & $\begin{array}{c}\text { RetTime } \\
\text { [min] }\end{array}$ & Type & $\begin{array}{l}\text { Width } \\
\text { [min] }\end{array}$ & $\begin{array}{c}\text { Area } \\
{\left[\mathrm{mAU}^{*} \mathrm{~S}\right]}\end{array}$ & $\begin{array}{l}\text { Height } \\
\text { [mAU] }\end{array}$ & $\begin{array}{c}\text { Area } \\
8\end{array}$ \\
\hline & -10 & & & & 10 & \\
\hline 1 & 4.916 & $\mathrm{BP}$ & 0.1032 & $1.16210 \mathrm{e} 4$ & 1852.77625 & 97.4902 \\
\hline 2 & 5.999 & $\mathrm{BP}$ & 0.3481 & 299.17227 & 10.44582 & 2.5098 \\
\hline Total & : & & & $1.19202 \mathrm{e} 4$ & 1863.22207 & \\
\hline
\end{tabular}

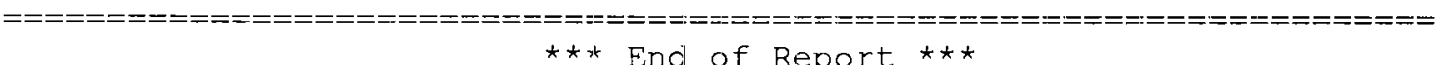

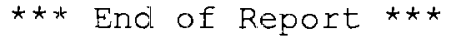



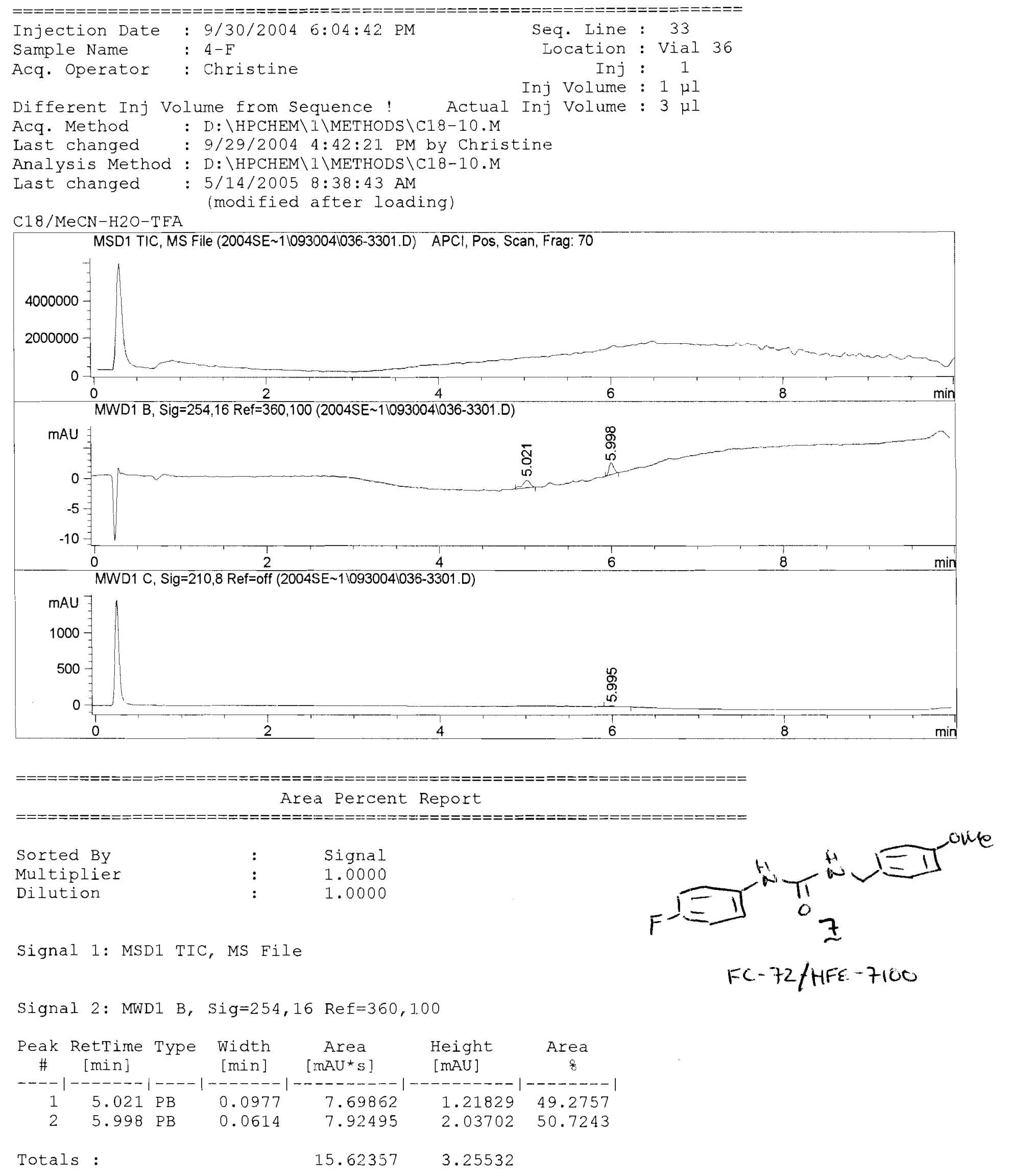


\begin{tabular}{|c|c|c|c|c|c|c|}
\hline $\begin{array}{c}\text { Peak } \\
\#\end{array}$ & $\begin{array}{c}\text { RetTime } \\
\text { [min] }\end{array}$ & Type & $\begin{array}{l}\text { Width } \\
\text { [min] }\end{array}$ & $\begin{array}{c}\text { Area } \\
{\left[\mathrm{mAU}{ }^{\star} \mathrm{S}\right]}\end{array}$ & $\begin{array}{l}\text { Height } \\
\text { [mAU] }\end{array}$ & $\begin{array}{c}\text { Area } \\
\&\end{array}$ \\
\hline---- & $\mid-------$ & & $-------\mid$ & ---------1 & $--------\mid$ & $--------\mid$ \\
\hline 1 & 5.995 & $\mathrm{BB}$ & 0.0853 & 64.26727 & 10.82481 & 100.0000 \\
\hline Tota & ls : & & & 64.26727 & 10.82481 & \\
\hline
\end{tabular}

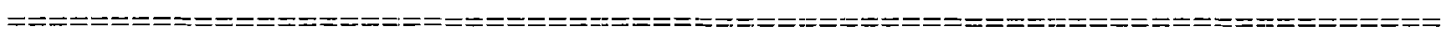
$\star \star \star$ End of Report $\star \star \star$ 


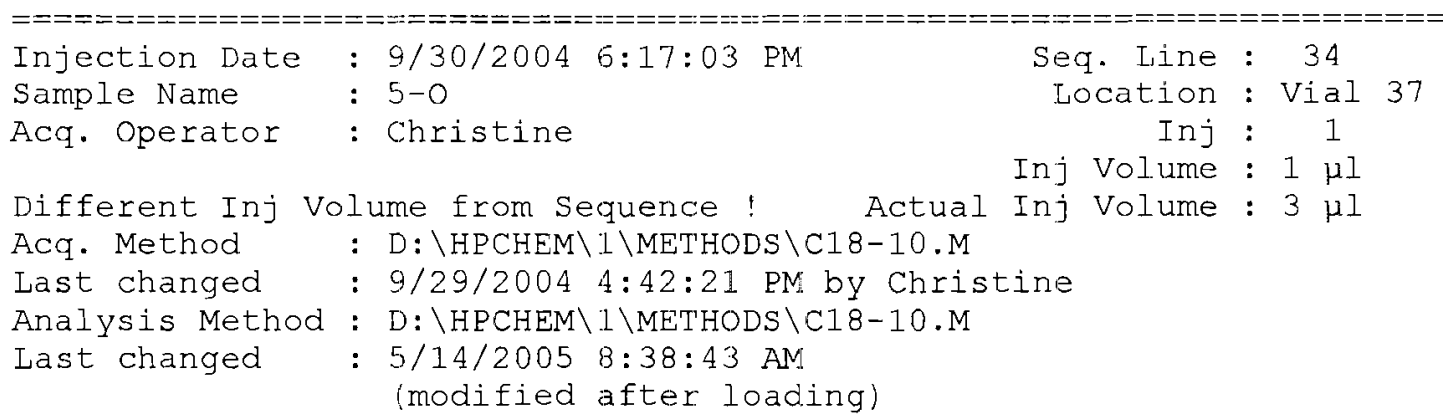

$\mathrm{C} 18 / \mathrm{MeCN}-\mathrm{H} 2 \mathrm{O}-\mathrm{TFA}$
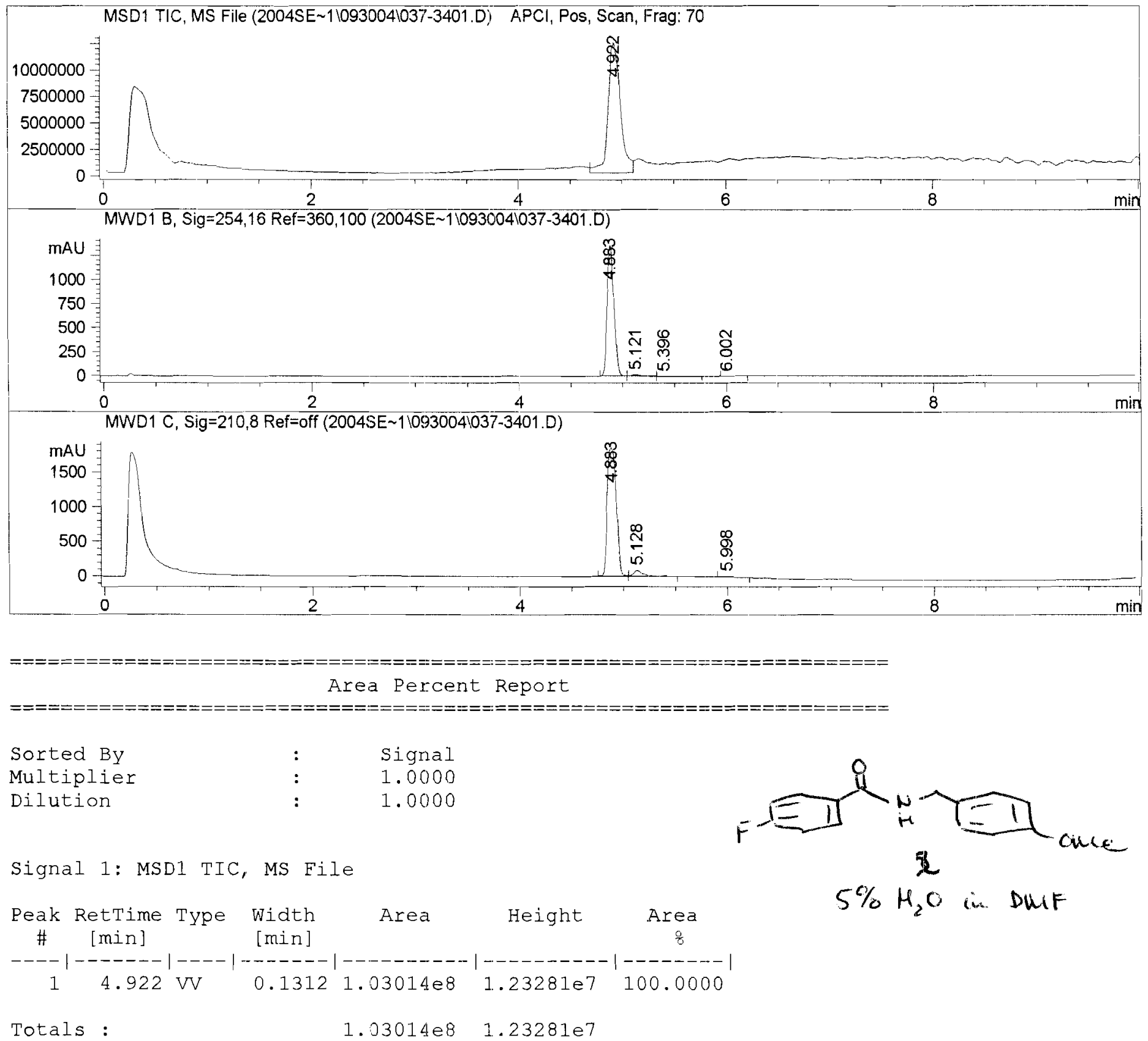

Signal 2: MWD1 B, Sig=254,16 Ref $=360,100$ 
Data File D: \HPCHEM $\backslash 1 \backslash$ DATA $\backslash 2004 \mathrm{SE} \sim 1 \backslash 093004 \backslash 037-3401 . \mathrm{D}$

\begin{tabular}{|c|c|c|c|c|c|c|}
\hline $\begin{array}{c}\text { Peak } \\
\#\end{array}$ & $\begin{array}{l}\text { RetTime } \\
\text { [min] }\end{array}$ & Type & $\begin{array}{l}\text { Width } \\
\text { [min] }\end{array}$ & $\begin{array}{c}\text { Area } \\
{\left[\mathrm{mAU}{ }^{\star} \mathrm{S}\right]}\end{array}$ & $\begin{array}{l}\text { Height } \\
\text { [mAU] }\end{array}$ & $\begin{array}{c}\text { Area } \\
\frac{8}{8}\end{array}$ \\
\hline 1 & 4.883 & BV & 0.0712 & 6208.31592 & 1361.29016 & 98.0828 \\
\hline 2 & 5.121 & VV & 0.0877 & 105.42694 & 17.65098 & 1.6656 \\
\hline 3 & 5.396 & VP & 0.0877 & 7.89886 & 1.28543 & 0.1248 \\
\hline 4 & 6.002 & $\mathrm{PP}$ & 0.0609 & 8.02417 & 1.99642 & 0.1268 \\
\hline Tota & s : & & & 6329.66589 & 1382.22299 & \\
\hline
\end{tabular}

Signal 3: MWD1 C, Sig=210,8 Ref=off

\begin{tabular}{|c|c|c|c|c|c|c|}
\hline $\begin{array}{c}\text { Peak } \\
\quad \#\end{array}$ & $\begin{array}{l}\text { RetTime } \\
\text { [min] }\end{array}$ & Type & $\begin{array}{l}\text { Width } \\
\text { [min] }\end{array}$ & $\begin{array}{c}\text { Area } \\
{\left[\mathrm{MAU}^{\star} \mathrm{S}\right]}\end{array}$ & $\begin{array}{l}\text { Height } \\
\text { [mAU] }\end{array}$ & $\begin{array}{c}\text { Area } \\
\frac{8}{8}\end{array}$ \\
\hline 1 & 4.883 & BV & 0.1009 & $1.12730 \mathrm{e} 4$ & 1855.07751 & 94.8788 \\
\hline 2 & 5.128 & VB & 0.0949 & 551.57861 & 83.73540 & 4.6423 \\
\hline 3 & 5.998 & $\mathrm{BB}$ & 0.0753 & 56.89879 & 10.48111 & 0.4789 \\
\hline
\end{tabular}

Totals : $\quad 1.18815 \mathrm{e} 4 \quad 1949.29402$ 

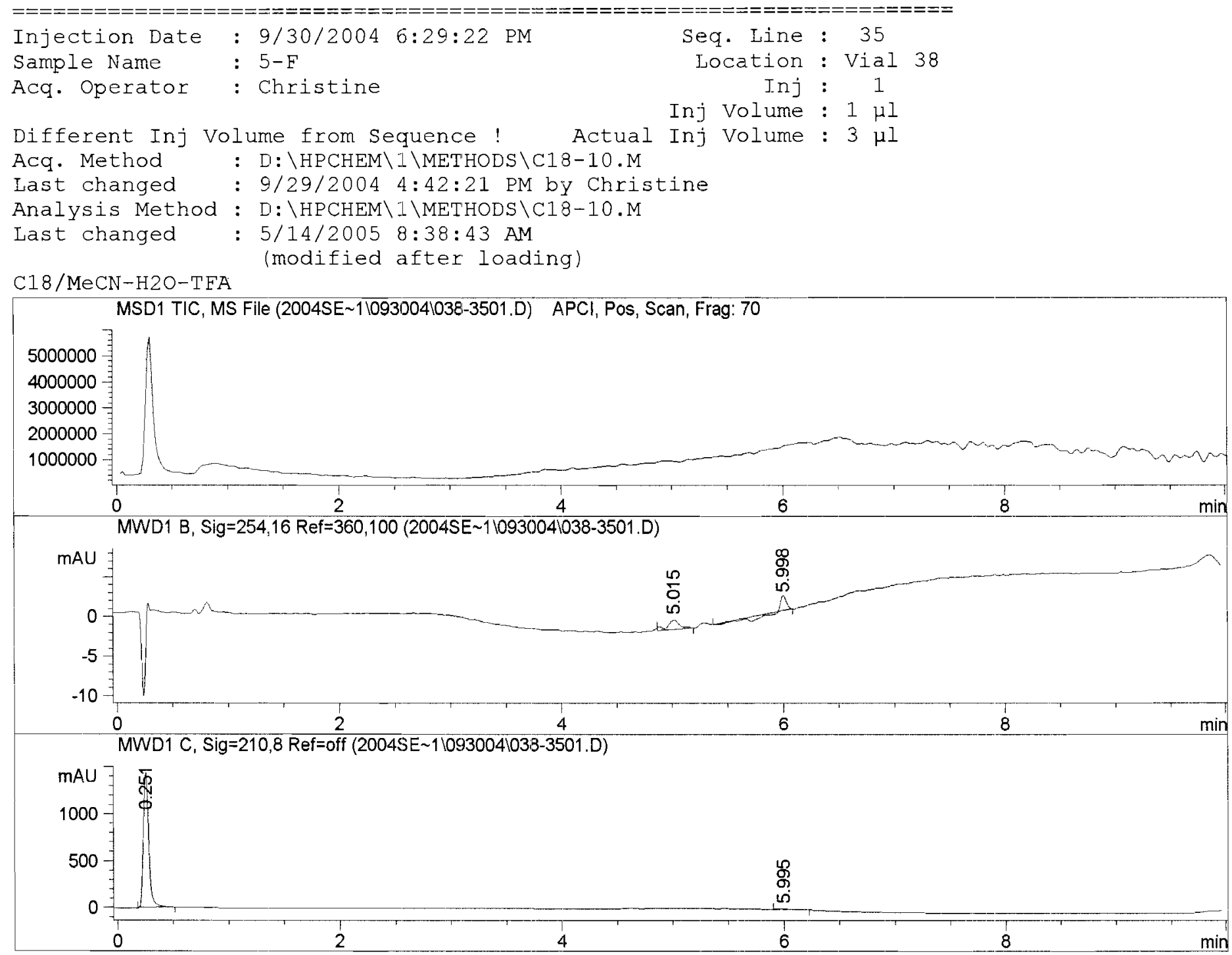

$\begin{array}{lll}\text { Sorted By } & : & \text { Signal } \\ \text { Multiplier } & : & 1.0000 \\ \text { Dilution } & : & 1.0000\end{array}$

Signal 1: MSDl TIC, MS File

Signal 2: MWD1 B, Sig=254,16 $\operatorname{Ref}=360,100$

\begin{tabular}{|c|c|c|c|c|c|c|}
\hline $\begin{array}{c}\text { Peak } \\
\#\end{array}$ & $\begin{array}{c}\text { RetTime } \\
\text { [min] }\end{array}$ & Type & $\begin{array}{l}\text { Width } \\
\text { [min] }\end{array}$ & $\begin{array}{c}\text { Area } \\
{\left[\mathrm{mAU}{ }^{\star} \mathrm{s}\right]}\end{array}$ & $\begin{array}{l}\text { Height } \\
{[\mathrm{mAU}]}\end{array}$ & $\begin{array}{c}\text { Area } \\
\&\end{array}$ \\
\hline & & & & ------- & & \\
\hline 1 & 5.015 & $\mathrm{BP}$ & 0.1154 & 9.01001 & 1.17574 & 49 \\
\hline 2 & 5.998 & $\mathrm{VP}$ & 0.0696 & 9.25356 & 1.94297 & 50.6668 \\
\hline
\end{tabular}

Totals :

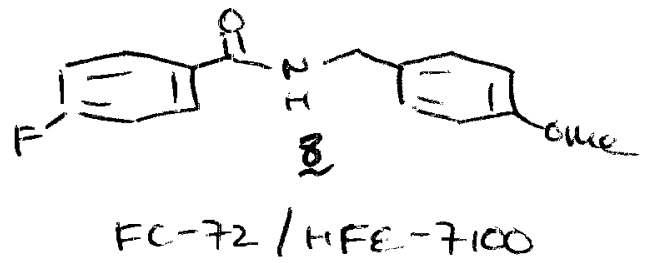

$F C-72 / H F E-7100$ 
Signal 3: MWD1 C, Sig=210,8 Ref=off

\begin{tabular}{|c|c|c|c|c|c|c|}
\hline $\begin{array}{c}\text { Peak } \\
\quad \#\end{array}$ & $\begin{array}{c}\text { RetTime } \\
\text { [min] }\end{array}$ & Type & $\begin{array}{l}\text { Width } \\
\text { [min] }\end{array}$ & $\begin{array}{c}\text { Area } \\
{\left[\mathrm{mAU}{ }^{\star} \mathrm{s}\right]}\end{array}$ & $\begin{array}{l}\text { Height } \\
\text { [mAU] }\end{array}$ & $\begin{array}{c}\text { Area } \\
8\end{array}$ \\
\hline & & & ------ & |-------- & & \\
\hline 1 & & $\mathrm{~PB}$ & & 4837.49316 & $1 \leq 45.44153$ & \\
\hline 2 & 5.99 & $\mathrm{BB}$ & 0 . & 66.07948 & 3377 & 76 \\
\hline
\end{tabular}

Totals :

$4903.57265 \quad 1455.97530$ 


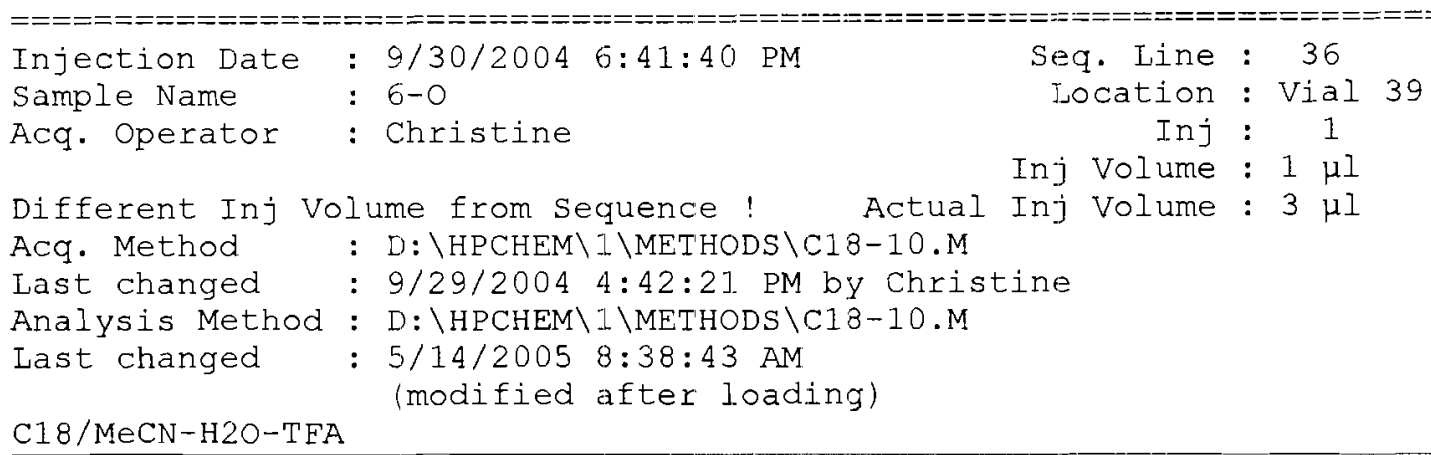

$\mathrm{C} 18 / \mathrm{MeCN}-\mathrm{H} 20-\mathrm{TEA}$

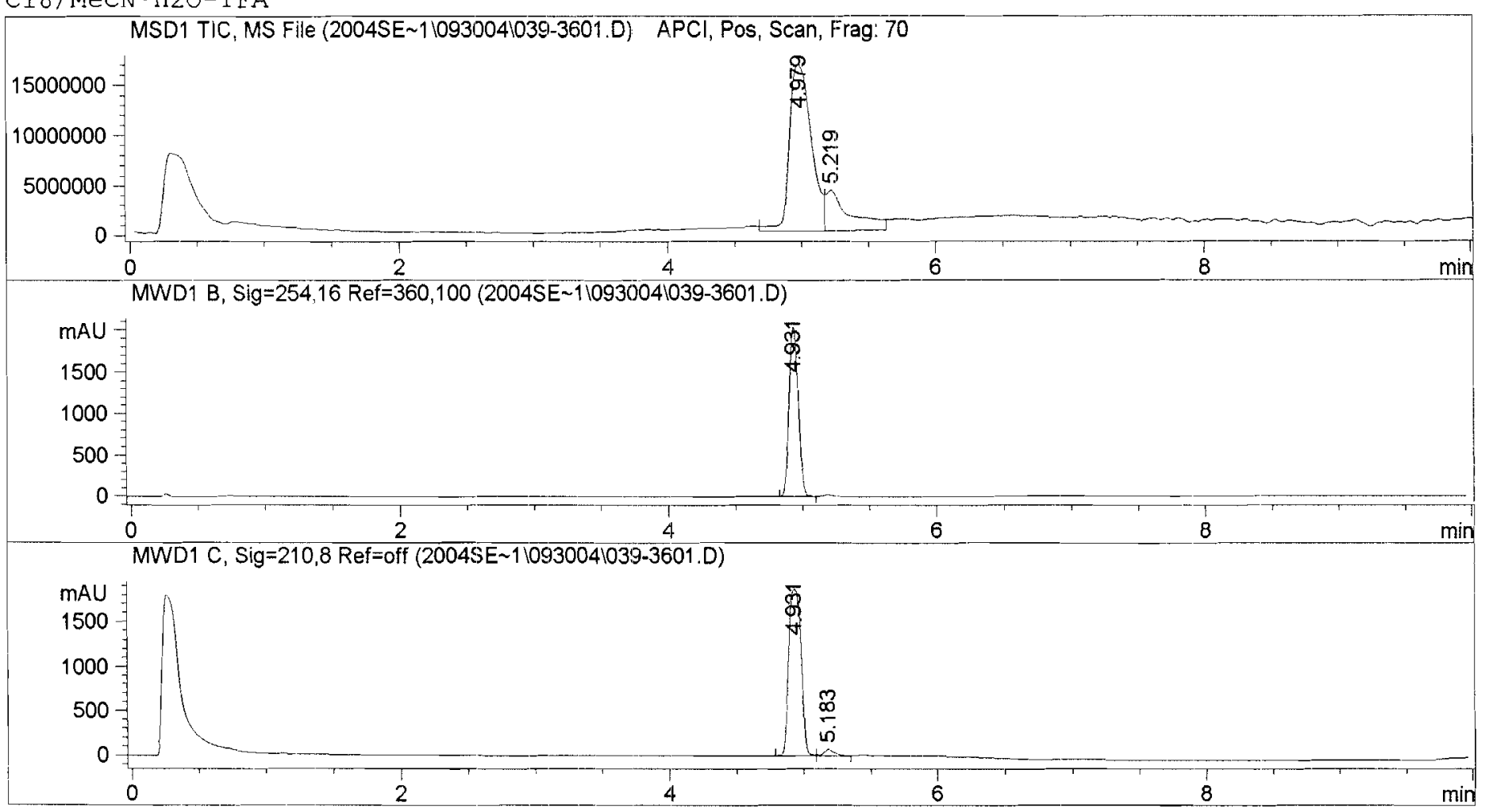

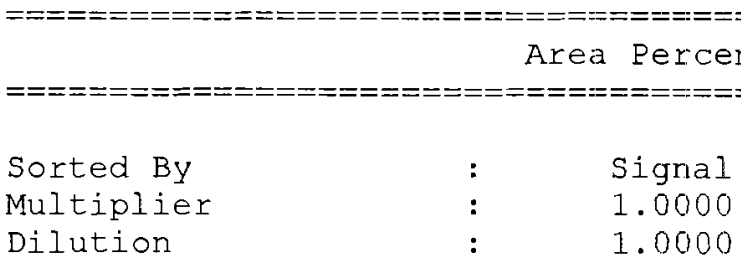

Dilution

$$
1.0000
$$

Signal 1: MSDI TIC, MS File

\begin{tabular}{|c|c|c|c|c|c|c|c|c|}
\hline $\begin{array}{c}\text { Peak } \\
\quad \#\end{array}$ & $\begin{array}{c}\text { RetTime } \\
\text { [min] }\end{array}$ & Type & $\begin{array}{l}\text { Width } \\
\text { [min] }\end{array}$ & Areal & Height & $\begin{array}{c}\text { Area } \\
\frac{8}{8}\end{array}$ & $5 \% \mathrm{H}_{2} \mathrm{O}$ & DULF \\
\hline----1 & $\mid------$ & $--\infty$ & ------- & |--.----- & $1---------$ & $--------\mid$ & & \\
\hline 1 & 4.979 & VV & 0.1787 & $1.90512 \mathrm{e} 8$ & $1.66170 \mathrm{e} 7$ & 78.3311 & & \\
\hline 2 & 5.219 & VV & 0.1730 & $5.27019 \mathrm{e} 7$ & $4.08553 e 6$ & 21.6689 & & \\
\hline Tota] & Ls: & & & 2.43214 e 8 & $2.07025 \mathrm{e} 7$ & & & \\
\hline
\end{tabular}


Signal 2: MWD1 B, Sig=254, 16 Ref $=360,1.00$

\begin{tabular}{|c|c|c|c|c|c|c|}
\hline $\begin{array}{c}\text { Peak } \\
\#\end{array}$ & $\begin{array}{c}\text { RetTime } \\
\text { [min] }\end{array}$ & Type & $\begin{array}{c}\text { Width } \\
\text { [min] }\end{array}$ & $\begin{array}{c}\text { Area } \\
{\left[\mathrm{mAU}{ }^{\star} \mathrm{s}\right]}\end{array}$ & $\begin{array}{l}\text { Height } \\
\text { [mAU] }\end{array}$ & $\begin{array}{c}\text { Area } \\
\frac{8}{8}\end{array}$ \\
\hline----1 & ------- & & ------ & ---------- & $\mid----------$ & $|----\cdots|$ \\
\hline 1 & 4.931 & BV & 0.0762 & 9849.14258 & 2043.58728 & 100.0000 \\
\hline Tota] & Ls: & & & 9849.14258 & 2.043 .58728 & \\
\hline
\end{tabular}

Signal 3: MWDl C, Sig=210,8 Ref=off

\begin{tabular}{|c|c|c|c|c|c|c|}
\hline $\begin{array}{c}\text { Peak } \\
\#\end{array}$ & $\begin{array}{l}\text { RetTime } \\
\quad[\mathrm{min}]\end{array}$ & Type & $\begin{array}{l}\text { Width } \\
\text { [min] }\end{array}$ & $\begin{array}{c}\text { Area } \\
{\left[\mathrm{mAU}{ }^{\star} \mathrm{s}\right]}\end{array}$ & $\begin{array}{l}\text { Height } \\
\text { [mAU] }\end{array}$ & $\begin{array}{c}\text { Area } \\
8\end{array}$ \\
\hline-- & $-\cdots--1$ & & ------ & --------- & |-ーー-ー-ー-ー & $-----\cdots$ \\
\hline 1 & 4.931 & BV & 0.1074 & $1.20680 \mathrm{e} 4$ & $1.867: 52332$ & 95.9059 \\
\hline 2 & 5.183 & VV & 0.0937 & 515.16132 & 77.40072 & 4.0941 \\
\hline
\end{tabular}

Totals : $\quad 1.25831 e 4 \quad 1.944 .92403$ 


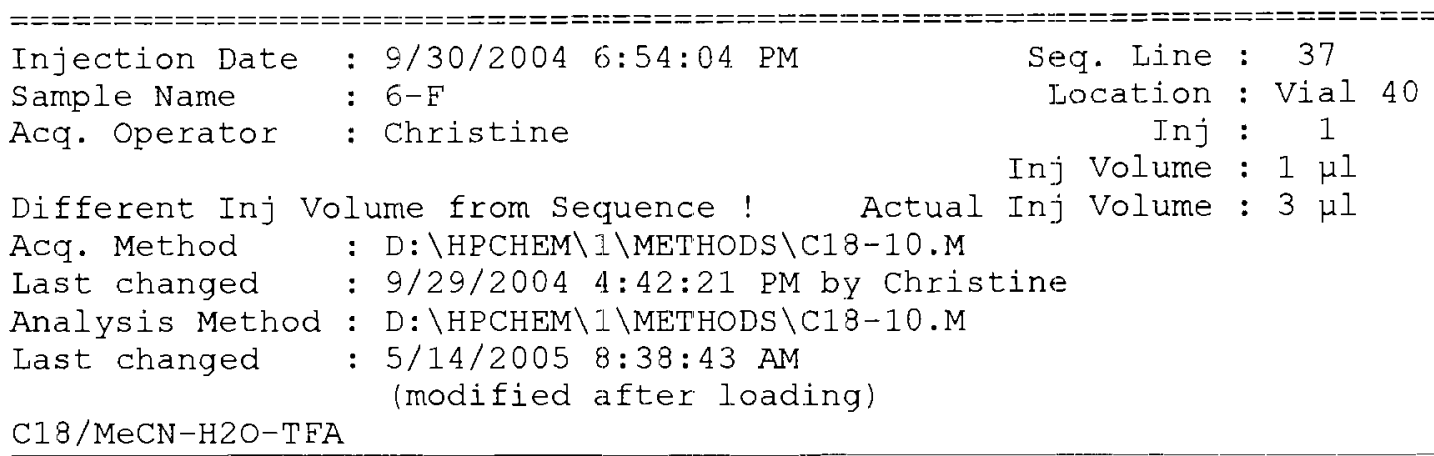

$\mathrm{C} 18 / \mathrm{MeCN}-\mathrm{H} 2 \mathrm{O}-\mathrm{TFA}$

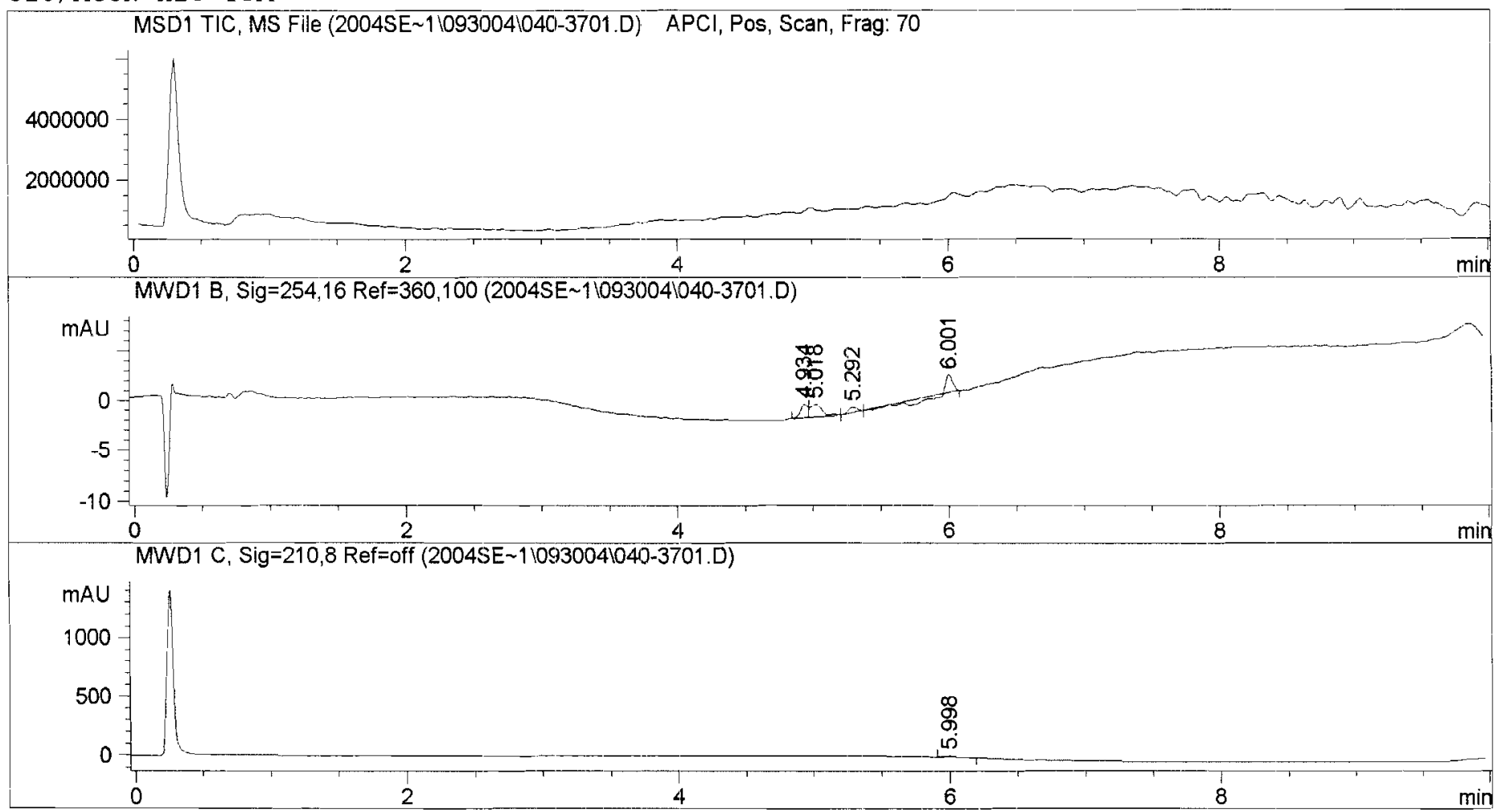

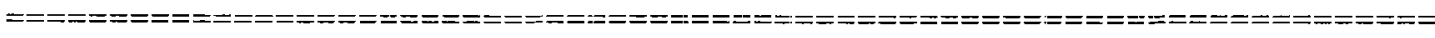

Area Percent Report

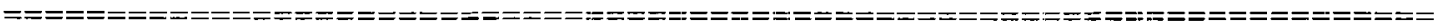

$\begin{array}{lll}\text { Sorted By } & : & \text { Signal } \\ \text { Multiplier } & : & 1.0000 \\ \text { Dilution } & : & 1.0000\end{array}$

Signal 1: MSD1 TIC, MS File

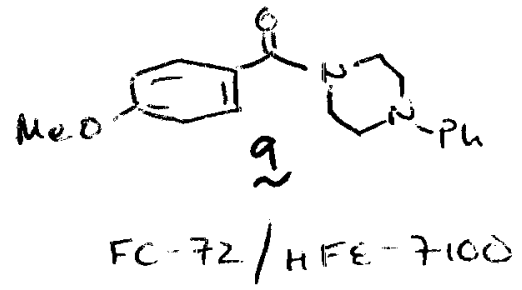

Signal 2: MWD1 B, Sig=254,16 Ref=360,100

$F C-72 / H F E-7100$

\begin{tabular}{|c|c|c|c|c|c|c|}
\hline $\begin{array}{c}\text { Peak } \\
\#\end{array}$ & $\begin{array}{l}\text { RetTime } \\
\text { [min] }\end{array}$ & Type & $\begin{array}{l}\text { Width } \\
\text { [min] }\end{array}$ & $\begin{array}{c}\text { Area } \\
{\left[\mathrm{mAU}^{\star} \mathrm{s}\right]}\end{array}$ & $\begin{array}{l}\text { Height } \\
\text { [mAU] }\end{array}$ & $\begin{array}{c}\text { Area } \\
8\end{array}$ \\
\hline 1 & 4.934 & BV & 0.0609 & 5.40533 & 1.34589 & 26.4362 \\
\hline 2 & 5.018 & VP & 0.0945 & 8.01970 & 1.25526 & 39.2225 \\
\hline 3 & 5.292 & VV & 0.0756 & 2.48379 & $5.40523 e-1$ & 12.1476 \\
\hline 4 & 6.001 & VP & 0.0431 & 4.53788 & 1.82195 & 22.1937 \\
\hline
\end{tabular}

Totals :

$20.44669 \quad 4.96362$ 
Signal 3: MWDl C, Sig=210,8 Ref=off

\begin{tabular}{|c|c|c|c|c|c|c|}
\hline $\begin{array}{c}\text { Peak } \\
\#\end{array}$ & $\begin{array}{l}\text { RetTime } \\
\text { [min] }\end{array}$ & Type & $\begin{array}{l}\text { Width } \\
\text { [min] }\end{array}$ & $\begin{array}{c}\text { Area } \\
{\left[m A U^{\star} s\right]}\end{array}$ & $\begin{array}{l}\text { Height } \\
\text { [mAU] }\end{array}$ & $\begin{array}{c}\text { Area } \\
\text { o }\end{array}$ \\
\hline 1 & 5.998 & $\mathrm{BB}$ & 0.0765 & 54.65451 & 10.20329 & 100.0000 \\
\hline Total & : & & & 54.65451 & 10.20329 & \\
\hline
\end{tabular}

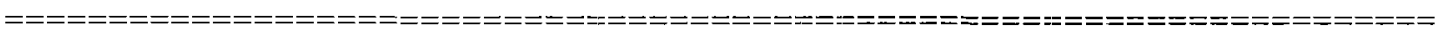
$\star \star \star$ End of Report *** 


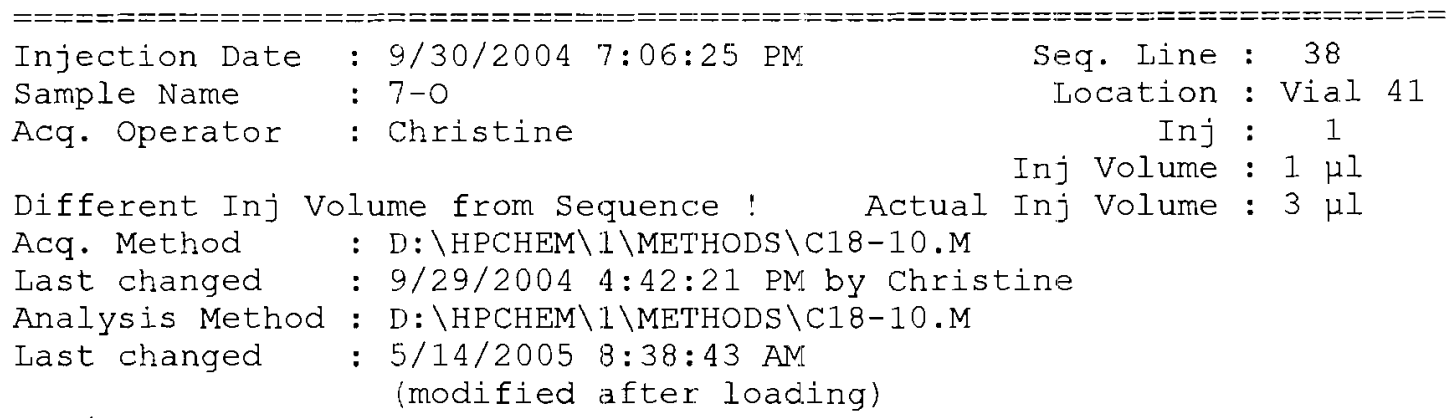

C18/MeCN-H2O-TEA

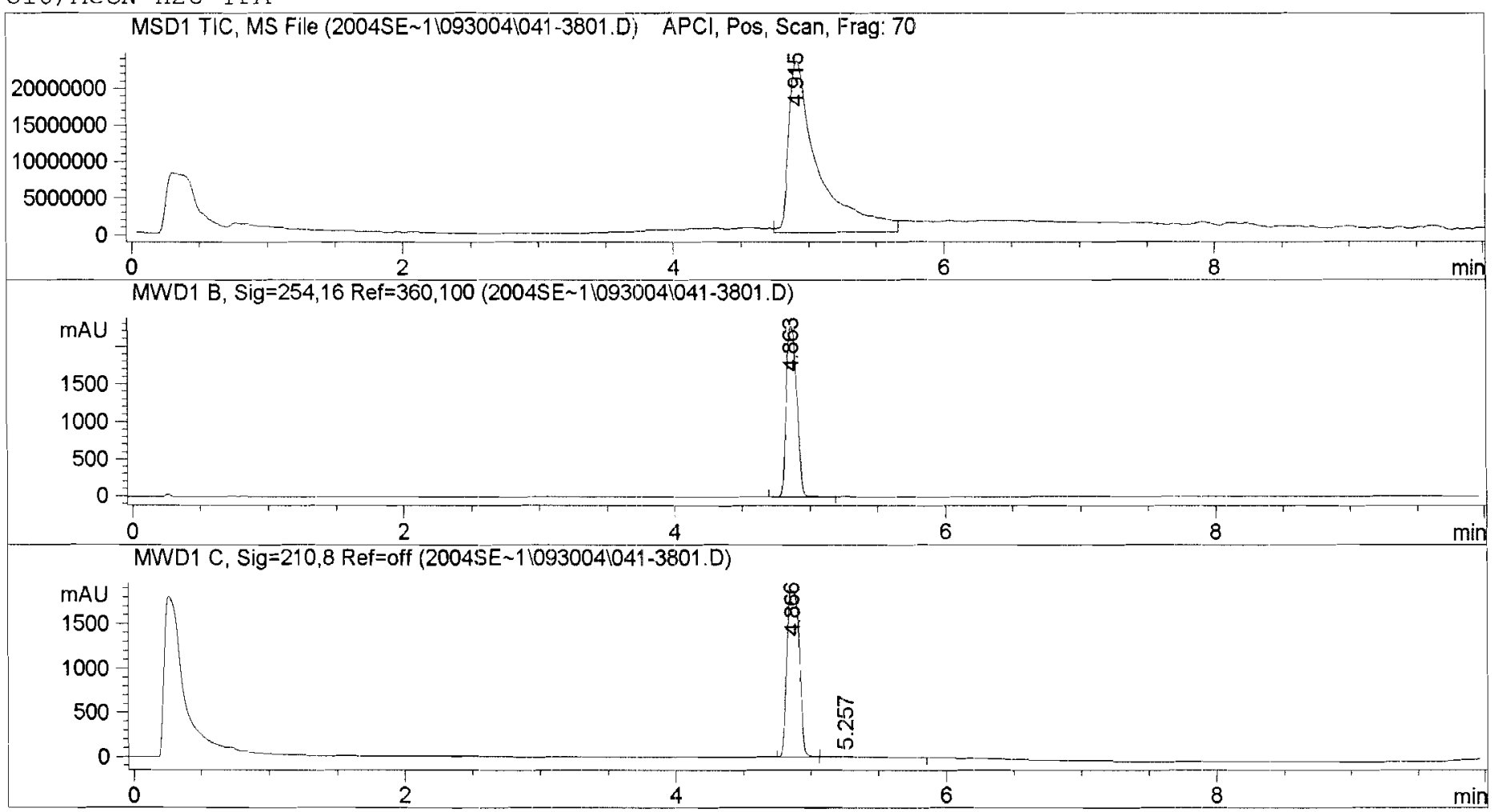

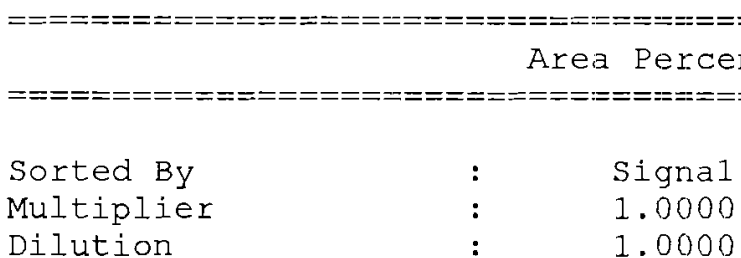

Signal 1: MSDL TIC, MS File

\begin{tabular}{|c|c|c|c|c|c|c|}
\hline $\begin{array}{c}\text { Peak } \\
\text { \# }\end{array}$ & $\begin{array}{l}\text { RetTime } \\
\text { [min] }\end{array}$ & Type & $\begin{array}{l}\text { Width } \\
\text { [min] }\end{array}$ & Area & Height & $\begin{array}{c}\text { Area } \\
\text { o }\end{array}$ \\
\hline 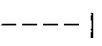 & ------1 & & ------ & --------1 & ---------1 & $|-------|$ \\
\hline 1 & 4.915 & VV & 0.2103 & $3.53772 \mathrm{e} 8$ & $2.33702 \mathrm{e} 7$ & 100.0000 \\
\hline Total & : & & & $3.53772 \mathrm{e} 8$ & $2.33702 \mathrm{e} 7$ & \\
\hline
\end{tabular}

Signal 2: MWD1 B, Sig=254,16 Ref=360, 100
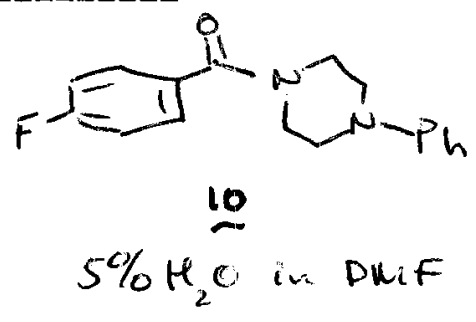
Data File D: \HPCHEM \I\DATA $\backslash 2004 \mathrm{SE} 1 \backslash 093004 \backslash 041-3801 . \mathrm{D}$

\begin{tabular}{|c|c|c|c|c|c|c|}
\hline $\begin{array}{c}\text { Peak } \\
\#\end{array}$ & $\begin{array}{c}\text { RetTime } \\
\text { [min] }\end{array}$ & Type & $\begin{array}{l}\text { Width } \\
\text { [min] }\end{array}$ & $\begin{array}{c}\text { Area } \\
{\left[m A U^{\star} s\right]}\end{array}$ & $\begin{array}{l}\text { Height } \\
\text { [mAU] }\end{array}$ & $\begin{array}{c}\text { Area } \\
8\end{array}$ \\
\hline 1 & 4.863 & VV & 0.0887 & $1.22041 \mathrm{e} 4$ & 2271.50537 & 100.0000 \\
\hline Total & : & & & $1.22041 \mathrm{e} 4$ & 2271.50537 & \\
\hline
\end{tabular}

Signal 3: MWD1 C, Sig=210,8 Ref=off

\begin{tabular}{|c|c|c|c|c|c|c|}
\hline $\begin{array}{c}\text { Peak } \\
\#\end{array}$ & $\begin{array}{c}\text { RetTime } \\
\text { [min] }\end{array}$ & Type & $\begin{array}{l}\text { Width } \\
\text { [min] }\end{array}$ & $\begin{array}{c}\text { Area } \\
{\left[\mathrm{mAU}^{\star} \mathrm{S}\right]}\end{array}$ & $\begin{array}{l}\text { Height } \\
{[\mathrm{mAU}]}\end{array}$ & $\begin{array}{c}\text { Area } \\
\frac{8}{8}\end{array}$ \\
\hline & & & & 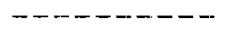 & & $\cdots$ \\
\hline 1 & 4.866 & BV & 0.1072 & $1.21027 \mathrm{e} 4$ & 1877.39526 & 97.4076 \\
\hline 2 & 5.257 & VV & 0.2284 & 322.09851 & 17.85936 & .5924 \\
\hline
\end{tabular}

Totals : $\quad 1.24248 e 4 \quad 1895.25462$ 


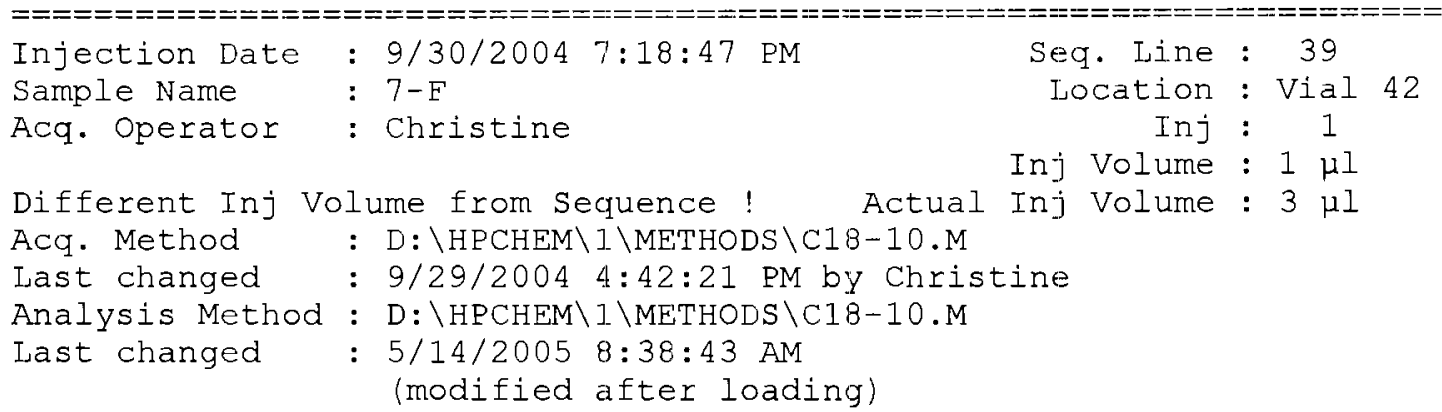

$\mathrm{C} 18 / \mathrm{MeCN}-\mathrm{H} 2 \mathrm{O}-\mathrm{TFA}$

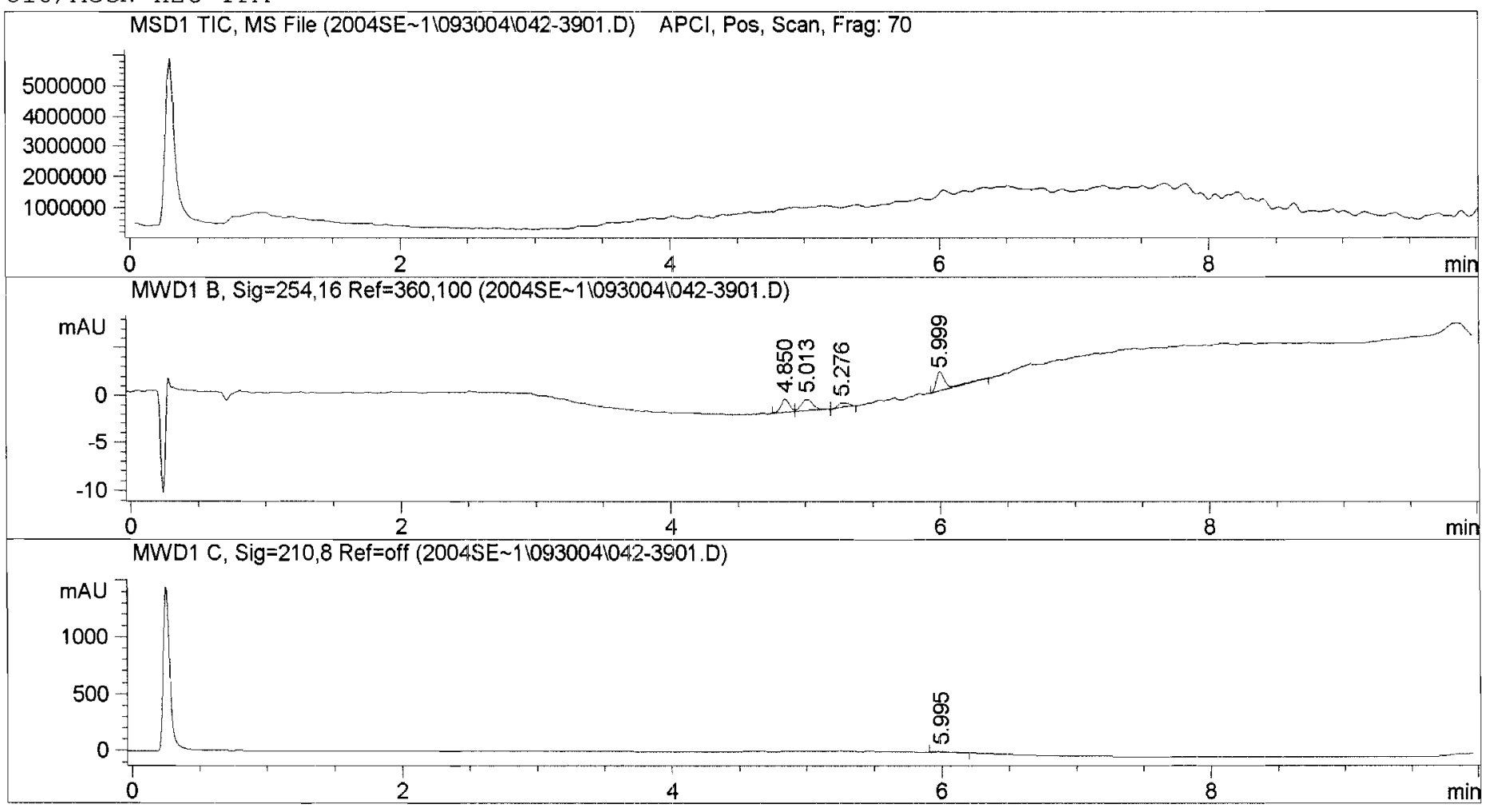

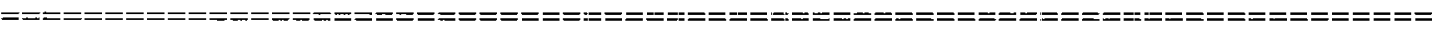

Area Percent Report

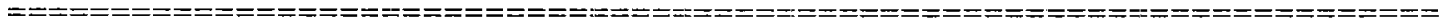

$\begin{array}{lll}\text { Sorted By } & : & \text { Signal } \\ \text { Multiplier } & : & 1.0000 \\ \text { Dilution } & : & 1.0000\end{array}$

Signal 1: MSD1 TIC, MS File

Signal 2: MWD1 B, Sig=254,16 Ref=360,100

\begin{tabular}{|c|c|c|c|c|c|c|}
\hline $\begin{array}{c}\text { Peak } \\
\#\end{array}$ & $\begin{array}{l}\text { RetTime } \\
\text { [min] }\end{array}$ & Type & $\begin{array}{c}\text { Width } \\
\text { [min] }\end{array}$ & $\begin{array}{c}\text { Area } \\
{\left[\mathrm{mAU}^{\star} \mathrm{s}\right]}\end{array}$ & $\begin{array}{l}\text { Height } \\
\text { [mAU] }\end{array}$ & $\begin{array}{c}\text { Area } \\
8\end{array}$ \\
\hline & & & & & & \\
\hline 1 & 4.850 & PV & 0.0672 & 6.51090 & 1.42893 & 24.8610 \\
\hline 2 & 5.013 & VP & 0.0993 & 8.01808 & 1.24301 & 30.6160 \\
\hline 3 & 5.276 & VP & 0.0783 & 2.85848 & $5.35386 e-1$ & 10.9147 \\
\hline 4 & 5.999 & $\mathrm{~PB}$ & 0.0676 & 8.80174 & 1.98914 & 33.6083 \\
\hline
\end{tabular}

Totals :

$26.18921 \quad 5.19647$

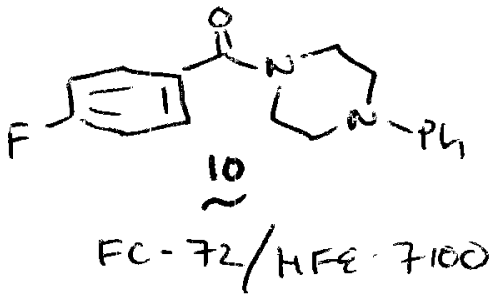


Signal 3: MWD1 C, Sig=210,8 Fef=off

\begin{tabular}{|c|c|c|c|c|c|c|}
\hline $\begin{array}{c}\text { Peak } \\
\#\end{array}$ & $\begin{array}{c}\text { RetTime } \\
\text { [min] }\end{array}$ & Type & $\begin{array}{l}\text { Width } \\
\text { [min] }\end{array}$ & $\begin{array}{c}\text { Area } \\
{\left[\mathrm{mAU}^{\star} \mathrm{s}\right]}\end{array}$ & $\begin{array}{l}\text { Height } \\
\text { [mAU] }\end{array}$ & $\begin{array}{c}\text { Area } \\
\frac{0}{b}\end{array}$ \\
\hline---1 & -------- & --- & ------- & ーーーーーーーーー & 904734 & -------1 \\
\hline Total & : & & & 57.94109 & 9.94734 & \\
\hline
\end{tabular}

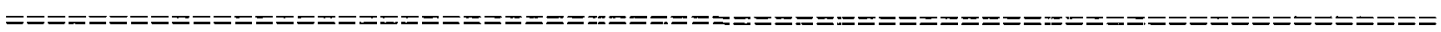
$\star \star *$ End of Report $\star \star \star ~$ 

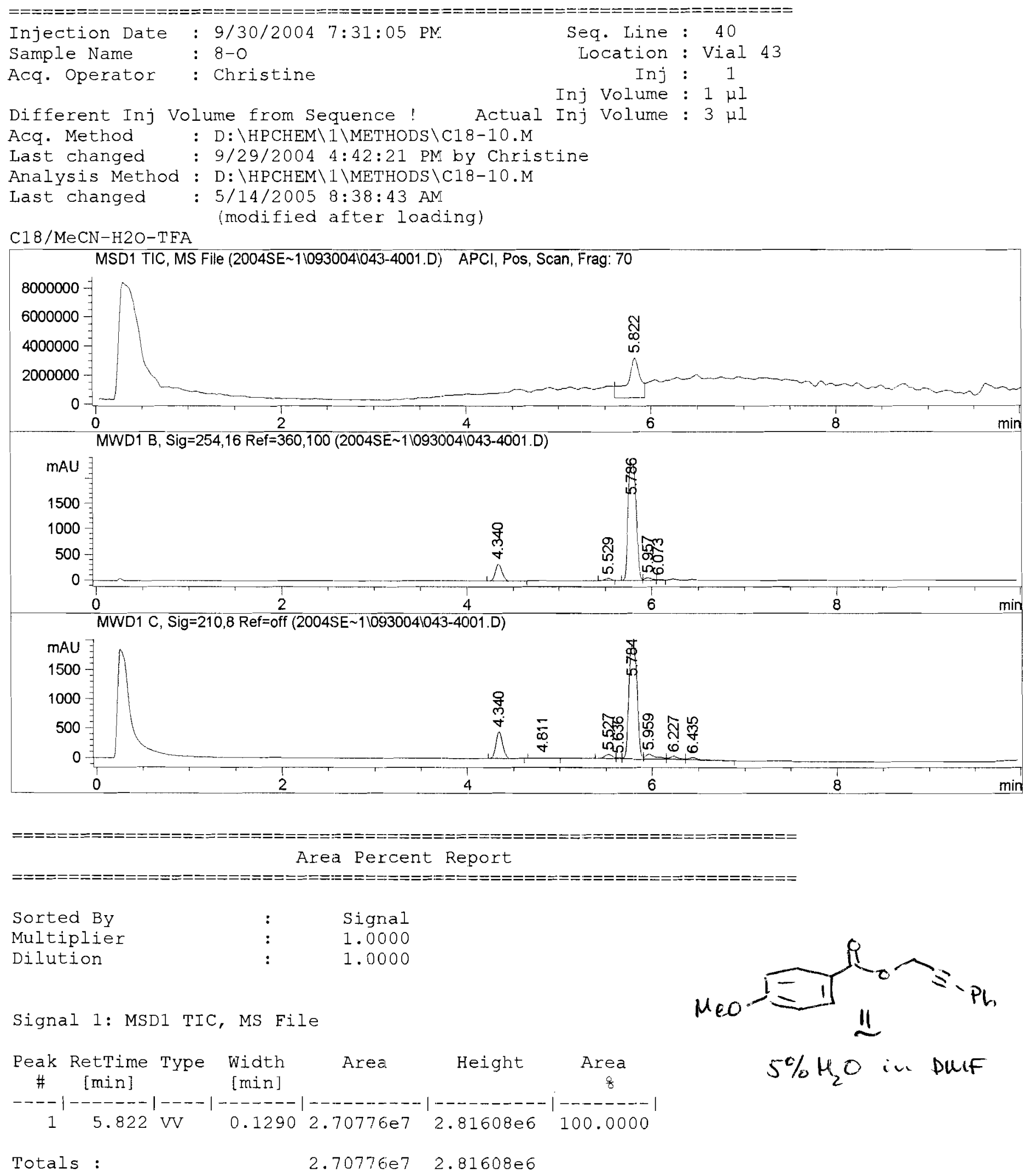

Signal 2: MWD1 B, Sig=254,16 Ref $=360,100$ 
Data File D: \HPCHEM $\backslash \backslash$ DATA $\backslash 2004 \mathrm{SE} \sim 1 \backslash 093004 \backslash 043-4001 . D$

\begin{tabular}{|c|c|c|c|c|c|c|}
\hline $\begin{array}{c}\text { Peak } \\
\#\end{array}$ & $\begin{array}{c}\text { RetTime } \\
\text { [min] }\end{array}$ & Type & $\begin{array}{l}\text { Width } \\
\text { [min] }\end{array}$ & $\begin{array}{c}\text { Area } \\
{\left[\mathrm{mA} U^{\star} \mathrm{s}\right]}\end{array}$ & $\begin{array}{l}\text { Height } \\
\text { [mAU] }\end{array}$ & $\begin{array}{c}\text { Area } \\
\text { 号 }\end{array}$ \\
\hline---- & ------- & & ----- & $--\cdots-----$ & $---\cdots---1$ & $-\cdots----$ \\
\hline 1 & 4.340 & VP & 0.0780 & 1626.98071 & 327.07373 & 10.0639 \\
\hline 2 & 5.529 & VV & 0.0922 & 241.92744 & 42.62942 & L. 4965 \\
\hline 3 & 5.786 & VV & 0.1006 & $1.38634 \mathrm{e} 4$ & 2291.36865 & 85.7538 \\
\hline 4 & 5.957 & VV & 0.0798 & 340.34979 & 62.23316 & 2.1053 \\
\hline 5 & 6.073 & VV & 0.0698 & 93.86101 & 19.63498 & 0.5806 \\
\hline
\end{tabular}

Totals :

1. 61665 e4 2742.93994

Signal 3: MWD1 C, Sig=210,8 Ref=cif

\begin{tabular}{|c|c|c|c|c|c|c|}
\hline eak & $\begin{array}{c}\text { RetTime } \\
\text { [min] }\end{array}$ & Type & $\begin{array}{l}\text { Width } \\
\text { [min] }\end{array}$ & $\begin{array}{c}\text { Area } \\
{[\text { InAU*s ] }}\end{array}$ & $\begin{array}{l}\text { Height } \\
\text { [mAU] }\end{array}$ & $\begin{array}{c}\text { Area } \\
z\end{array}$ \\
\hline & & & ------ & ---------- & ---------- & -------- \\
\hline 1 & 4.340 & BV & 0.0791 & 2271.94678 & 448.57367 & 14.2278 \\
\hline 2 & 4.811 & $\mathrm{PP}$ & 0.0939 & 86.65076 & 14.46850 & 0.5426 \\
\hline 3 & 5.527 & BV & 0.0959 & 407.29141 & 67.99838 & 2.5506 \\
\hline 4 & 5.636 & VV & 0.0544 & 77.99684 & 21.47838 & 0.4884 \\
\hline 5 & 5.784 & VV & 0.1014 & $1.18071 \mathrm{e} 4$ & 1928.85364 & 73.9406 \\
\hline 6 & 5.959 & VV & 0.1133 & 682.38477 & 83.51903 & 4.2733 \\
\hline 7 & 6.227 & VV & 0.0990 & 364.51138 & 52.49294 & 2.2827 \\
\hline 8 & 6.435 & VB & 0.1095 & 270.49255 & 36.07886 & 1.6939 \\
\hline
\end{tabular}

Totals :

$1.59684 \mathrm{e} 4 \quad 2653.46340$ 


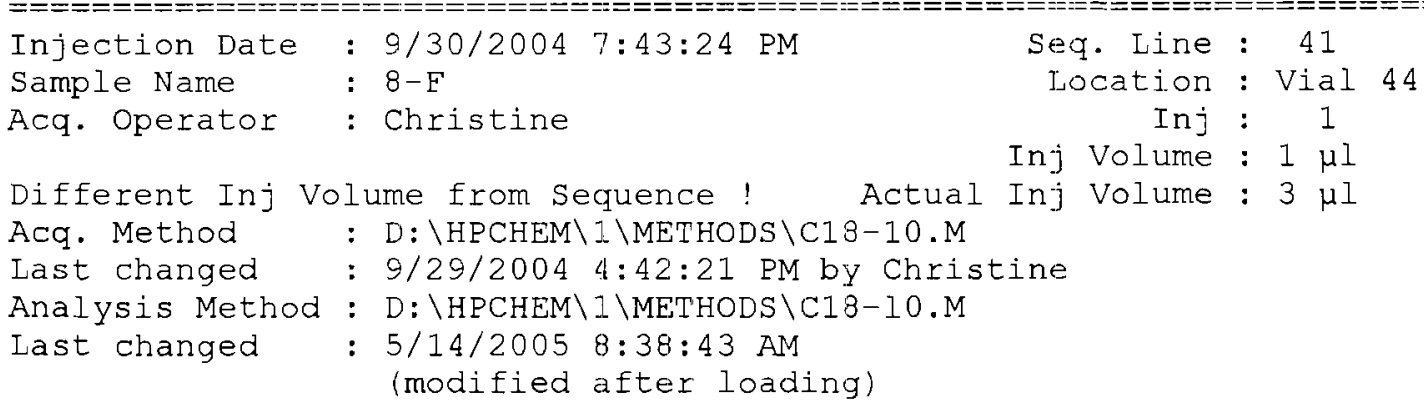

$\mathrm{C} 18 / \mathrm{MeCN}-\mathrm{H} 2 \mathrm{O}-\mathrm{TFA}$

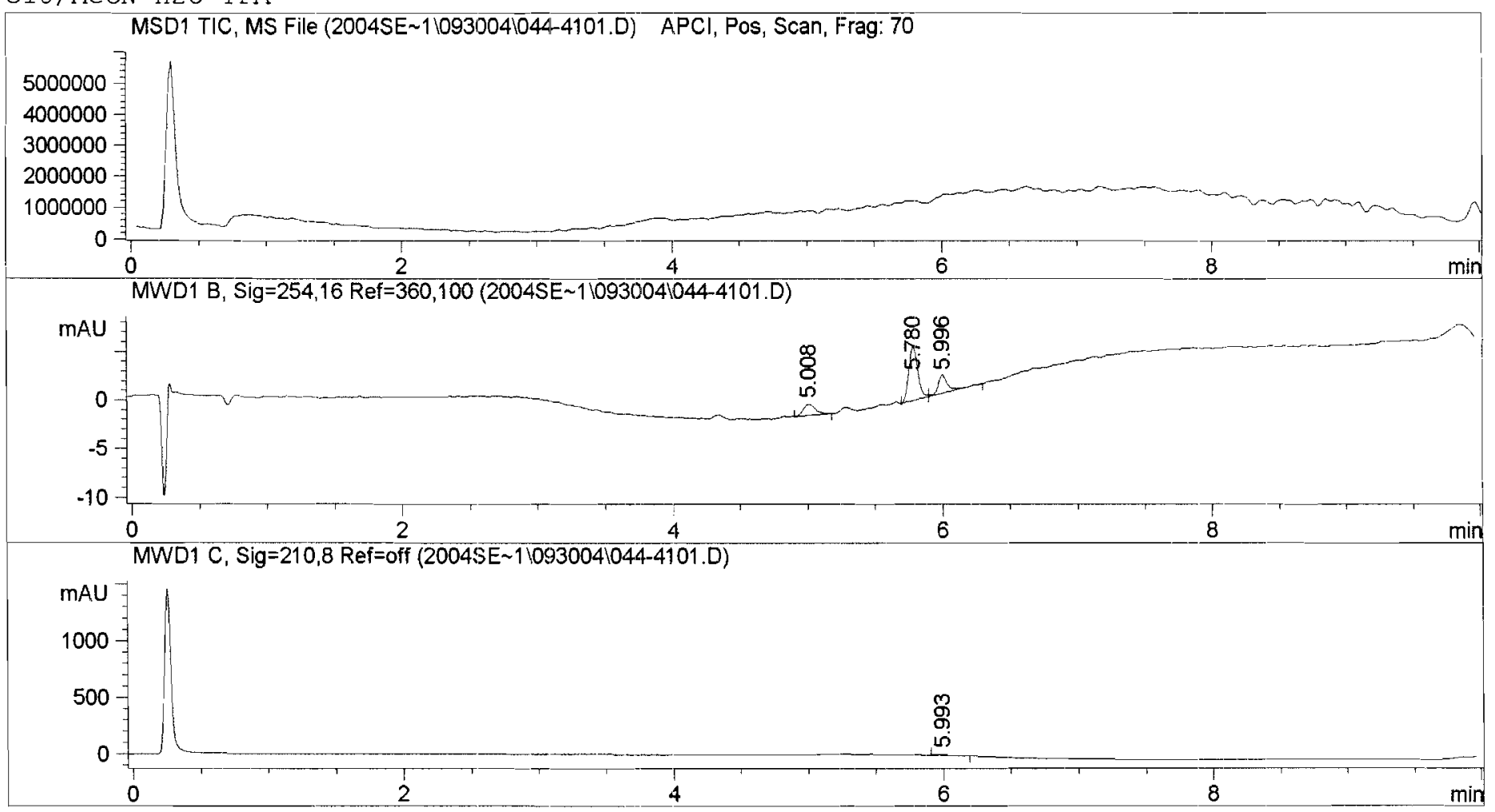

Area Percent Report

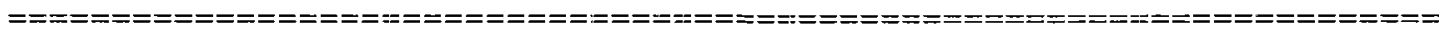

$\begin{array}{lll}\text { Sorted By } & : & \text { Signal } \\ \text { Multiplier } & : & 1.0000 \\ \text { Dilution } & : & 1.0000\end{array}$

Signal 1: MSD1 TIC, MS File

Signal 2: MWD1 B, Sig=254, 16 Ref $=360,100$

\begin{tabular}{|c|c|c|c|c|c|c|}
\hline $\begin{array}{c}\text { Peak } \\
\#\end{array}$ & $\begin{array}{c}\text { RetTime } \\
\text { [min] }\end{array}$ & Type & $\begin{array}{l}\text { Width } \\
\text { [min] }\end{array}$ & $\begin{array}{c}\text { Area } \\
{\left[\mathrm{mAU}^{\star} \mathrm{s}\right]}\end{array}$ & $\begin{array}{l}\text { Height } \\
\text { [mAU] }\end{array}$ & $\begin{array}{c}\text { Area } \\
\qquad z\end{array}$ \\
\hline & & & & & & \\
\hline 1 & 5.008 & $\mathrm{PP}$ & 0.0927 & 6.98001 & 1.15201 & 5567 \\
\hline 2 & 5.780 & PV & 0.0734 & 26.33662 & 5.54935 & 62.4709 \\
\hline 3 & 5.996 & VP & 0.0673 & 8.84160 & 1.93416 & 20.9724 \\
\hline
\end{tabular}

Totals :

42.15823

8.63553

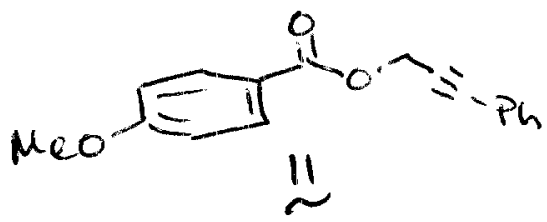

$F C=72 / H F E-7100$ 
Signal 3: MWD1 C, Sig=210,8 Ref=off

\begin{tabular}{|c|c|c|c|c|c|c|}
\hline $\begin{array}{c}\text { Peak } \\
\#\end{array}$ & $\begin{array}{l}\text { RetTime } \\
\quad[\mathrm{min}]\end{array}$ & Type & $\begin{array}{c}\text { width } \\
\text { [min] }\end{array}$ & $\begin{array}{c}\text { Area } \\
{\left[\mathrm{mAU}^{\star} \mathrm{s}\right]}\end{array}$ & $\begin{array}{l}\text { Height } \\
{[\mathrm{mAU}]}\end{array}$ & $\begin{array}{c}\text { Area } \\
\&\end{array}$ \\
\hline & - - - - - & & ------ & $--\cdots-\ldots---1$ & -------- & -------- \\
\hline 1 & 5.993 & $B B$ & 0.0734 & 46.64635 & 9.16188 & 100.0000 \\
\hline Total & Ls: & & & 46.64635 & 9.16188 & \\
\hline
\end{tabular}

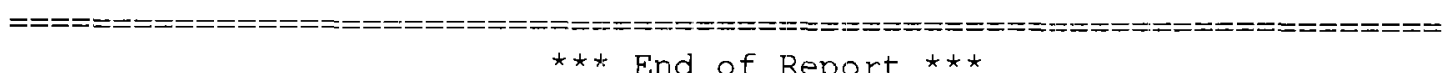



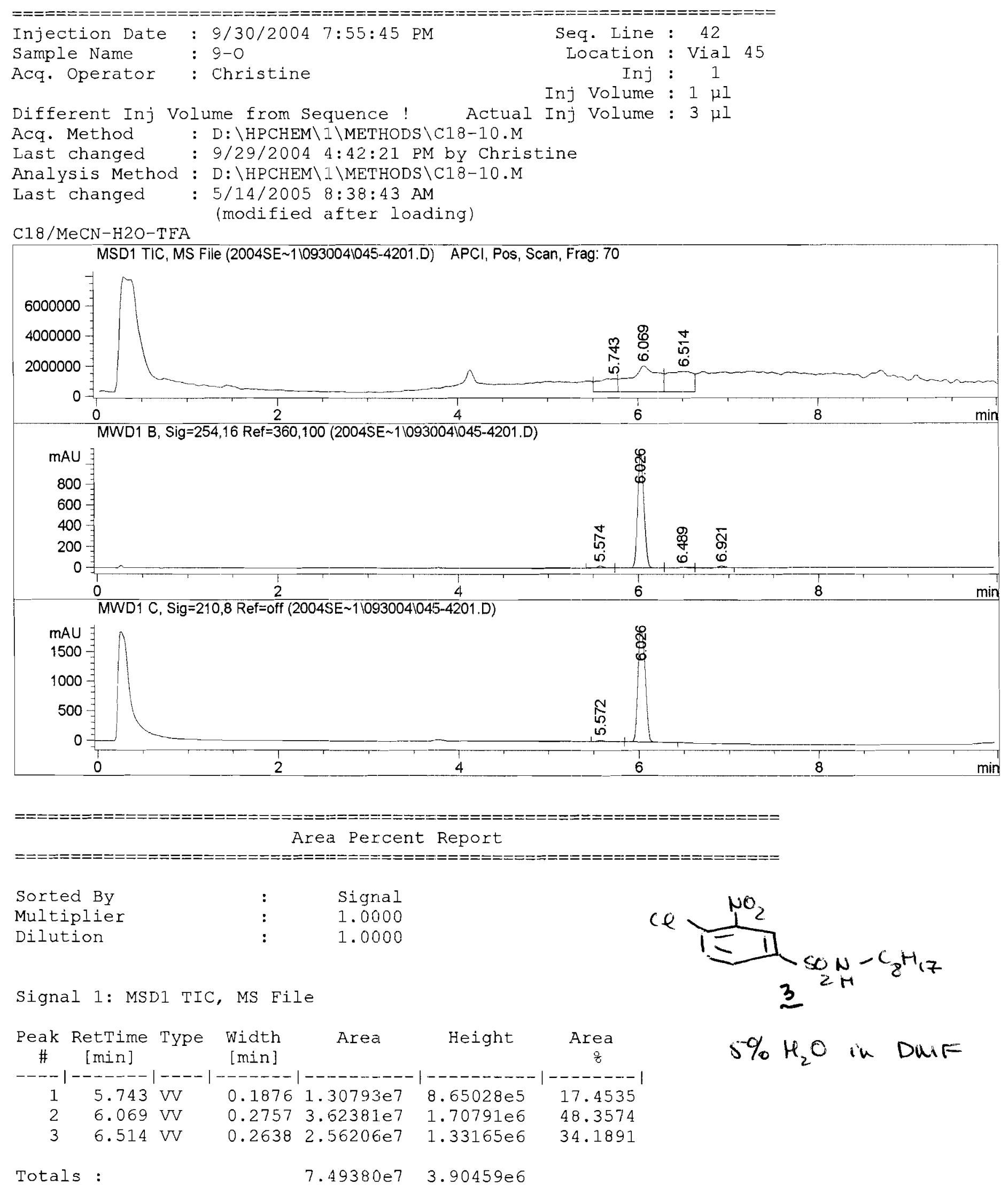
Signal 2: MWD1 B, Sig=254,16 Ref:=360,100

\begin{tabular}{|c|c|c|c|c|c|c|}
\hline $\begin{array}{c}\text { Peak } \\
\#\end{array}$ & $\begin{array}{c}\text { RetTime } \\
\text { [min] }\end{array}$ & Type & $\begin{array}{l}\text { Width } \\
\text { [min] }\end{array}$ & $\begin{array}{c}\text { Area } \\
{\left[\mathrm{nAU} U^{\star} \mathrm{S}\right]}\end{array}$ & $\begin{array}{l}\text { Height } \\
\text { [mAU] }\end{array}$ & $\begin{array}{c}\text { Area } \\
\frac{8}{8}\end{array}$ \\
\hline- & & & & $-6-2-1$ & --------- & -------- \\
\hline 1 & 5.574 & PP & 0.0739 & 86.21896 & 17.99792 & 1.5863 \\
\hline 2 & 6.026 & VV & 0.0754 & 5232.37256 & 1101.58374 & 96.2667 \\
\hline 3 & 6.489 & VV & 0.1173 & 26.11072 & 3.13154 & 0.4804 \\
\hline 4 & 6.921 & VB & 0.0956 & 90.58657 & 14.36304 & 1.6666 \\
\hline
\end{tabular}

Totals : $5435.28880 \quad 1137.07623$

Signal 3: MWD1 C, $S i g=210,8$ Ref=ceff

\begin{tabular}{|c|c|c|c|c|c|c|}
\hline $\begin{array}{c}\text { Peak } \\
\#\end{array}$ & $\begin{array}{c}\text { RetTime } \\
\text { [min] }\end{array}$ & Type & $\begin{array}{l}\text { Width } \\
\text { [min] }\end{array}$ & $\begin{array}{c}\text { Area } \\
{\left[\mathrm{InAU}^{*} \mathrm{~S}\right]}\end{array}$ & $\begin{array}{l}\text { Height } \\
\text { [mAU] }\end{array}$ & $\begin{array}{c}\text { Area } \\
\frac{8}{z}\end{array}$ \\
\hline---- & & & & | $-\cdots------$ & & --------1 \\
\hline 1 & 5.572 & BV & 0.1029 & 173.31221 & 23.82379 & 1.5909 \\
\hline 2 & 6.026 & VP & 0.0926 & $1.07207 e^{4}$ & 1878.94873 & 98.4091 \\
\hline
\end{tabular}

Totals :

$1.08940 \mathrm{e} \quad 1902.77252$

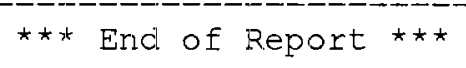




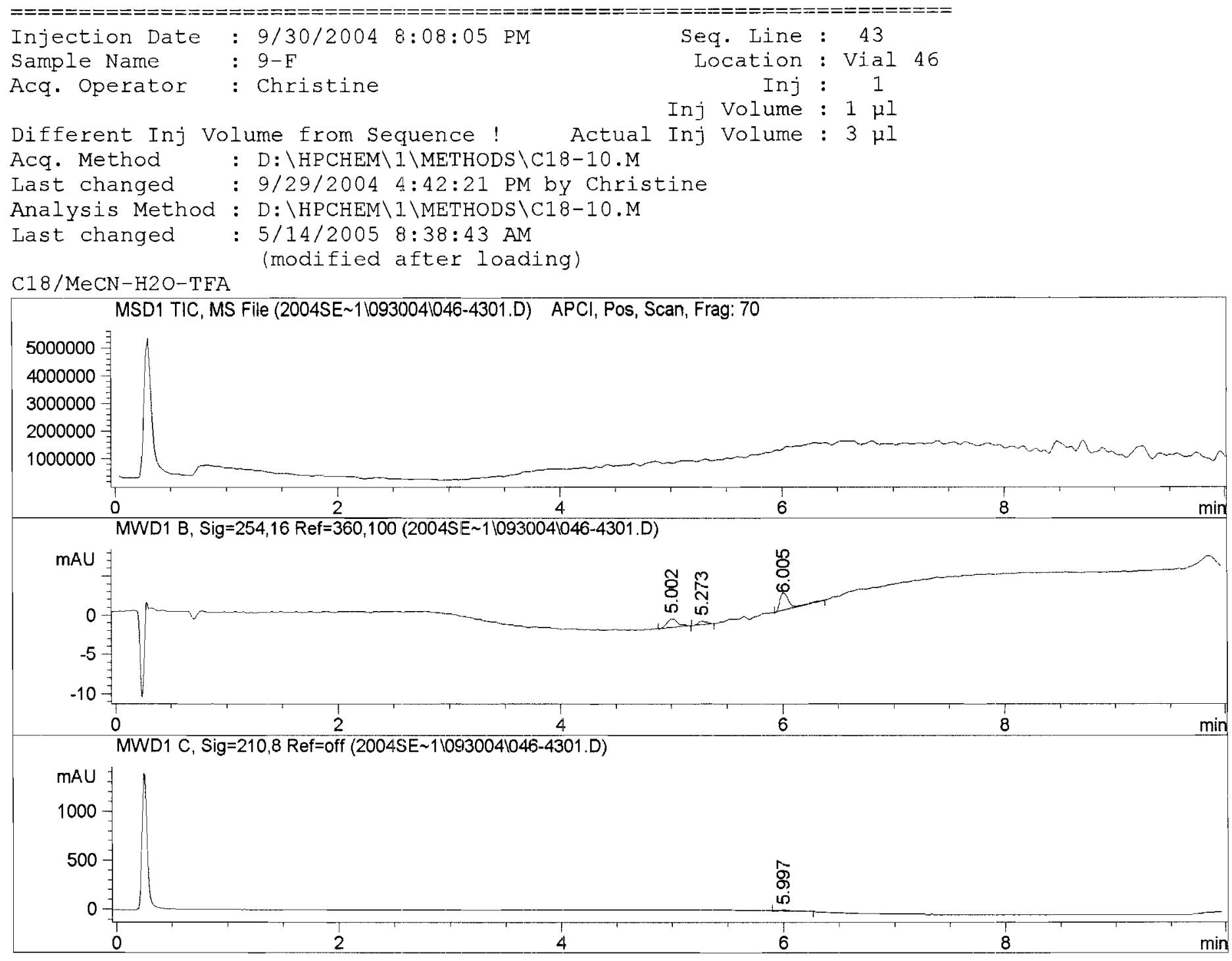

Area Percent Report

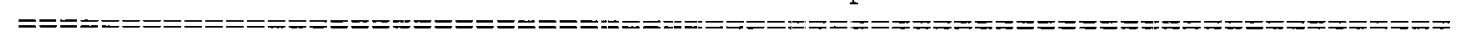

Sorted By

Multiplier

Dilution
Signal

1.0000

1.0000

Signal 1: MSD1 TIC, MS File

Signal 2: MWD1 B, Sig=254,16 Ref=360, 100

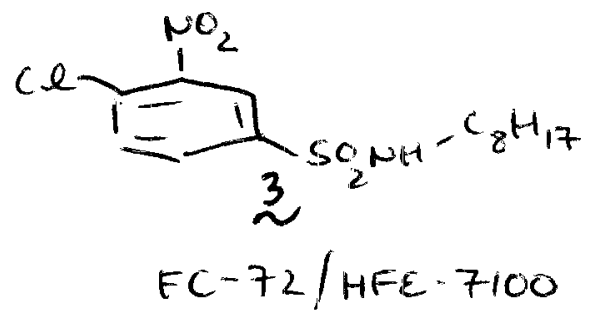

Totals :
18.43264
3.90031 
Signal 3: MWD1 C, Sig=210,8 Ref=off

\begin{tabular}{|c|c|c|c|c|c|c|}
\hline $\begin{array}{c}\text { Peak } \\
\#\end{array}$ & $\begin{array}{l}\text { RetTime } \\
\text { [min] }\end{array}$ & Type & $\begin{array}{l}\text { Width } \\
\text { [min] }\end{array}$ & $\begin{array}{c}\text { Area } \\
{\left[m U^{*} s\right]}\end{array}$ & $\begin{array}{l}\text { Height } \\
\text { [nAU] }\end{array}$ & $\begin{array}{c}\text { Area } \\
\frac{8}{8}\end{array}$ \\
\hline 1 & 5.997 & $\mathrm{BB}$ & 0.0988 & 75.22890 & 10.60019 & 100.0000 \\
\hline Total & : & & & 75.22890 & 10.60019 & \\
\hline
\end{tabular}

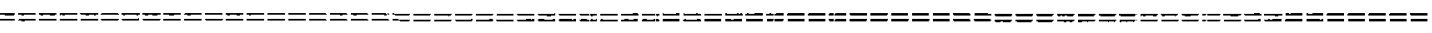

$\star \star \star$ End of Report $\star \star \star$ 


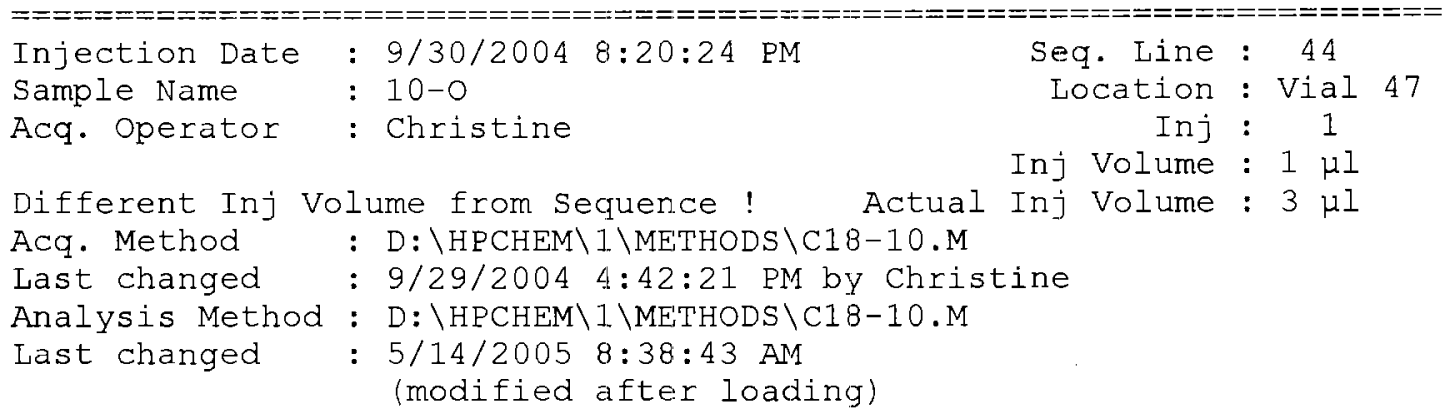

$\mathrm{C} 18 / \mathrm{MeCN}-\mathrm{H} 2 \mathrm{O}-\mathrm{TFA}$
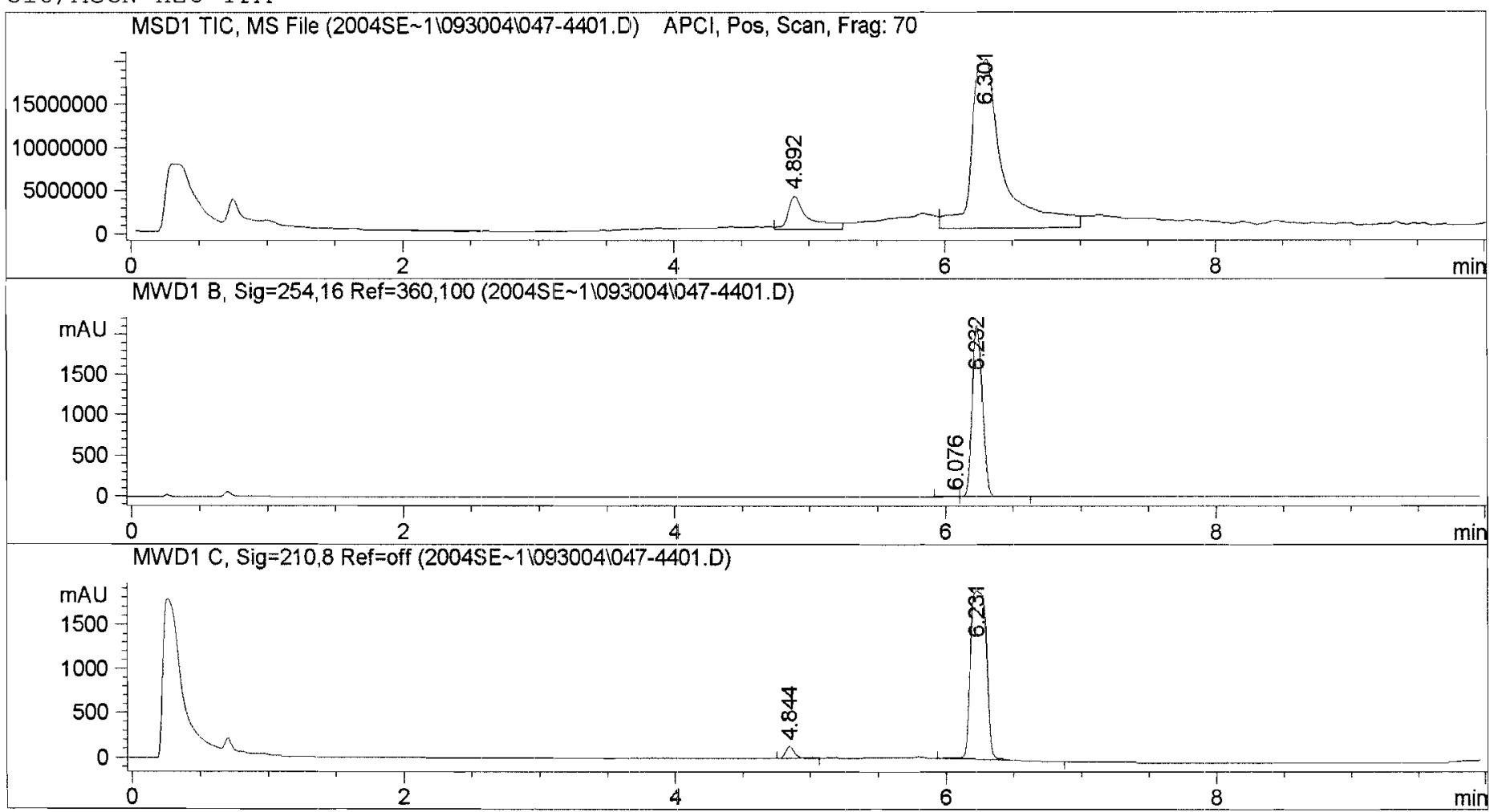

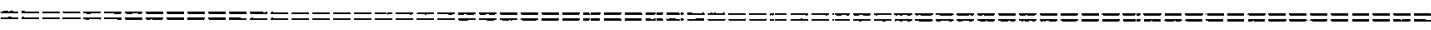

Area Percent Report

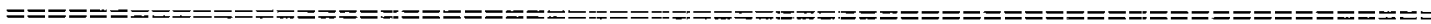

$\begin{array}{lll}\text { Sorted By } & : & \text { Signal } \\ \text { Multiplier } & : & 1.0000 \\ \text { Dilution } & : & 1.0000\end{array}$

$\mathrm{PPh}_{3}$

Signal 1: MSD1 TIC, MS File

$5 \% \mathrm{H}_{2} \mathrm{O}$ in DUAF

\begin{tabular}{|c|c|c|c|c|c|c|}
\hline $\begin{array}{c}\text { Peak } \\
\#\end{array}$ & $\begin{array}{c}\text { RetTime } \\
\text { [min] }\end{array}$ & Type & $\begin{array}{l}\text { Width } \\
{[\min ]}\end{array}$ & Area & Height & $\begin{array}{c}\text { Area } \\
\frac{8}{8}\end{array}$ \\
\hline & & & & & & \\
\hline 1 & 4.892 & VV & 0.1494 & $4.26734 \mathrm{e} 7$ & $3.92347 e 6$ & 11.710 \\
\hline 2 & 6.301 & VV & 0.2360 & $3.21745 e 8$ & $1.96536 \mathrm{e} 7$ & 88.2900 \\
\hline
\end{tabular}

Totals :

$$
3.64419 e 8 \quad 2.35771 \text { e } 7
$$


Signal 2: MWD1 B, Sig=254, 16 Ref=360, 100

\begin{tabular}{|c|c|c|c|c|c|c|}
\hline $\begin{array}{c}\text { Peak } \\
\#\end{array}$ & $\begin{array}{c}\text { RetTime } \\
\text { [min] }\end{array}$ & Type & $\begin{array}{c}\text { Width } \\
{[\mathrm{min}]}\end{array}$ & $\begin{array}{c}\text { Areal } \\
{\left[\mathrm{mAU}^{*} \mathrm{~s}\right]}\end{array}$ & $\begin{array}{l}\text { Height } \\
{[\mathrm{mAU}]}\end{array}$ & $\begin{array}{c}\text { Area } \\
\frac{8}{8}\end{array}$ \\
\hline & ----- & & & $-----\cdots$ & & \\
\hline 1 & & VV & & 7350 & 03 & \\
\hline 2 & 6.232 & B & 827 & $1.10196 \mathrm{e} 4$ & 2118 . & 99 \\
\hline
\end{tabular}

Totals :

$1.10465 \mathrm{e} 4 \quad 2122.60755$

Signal 3: MWD1 C, Sig=210,8 Ref=off

\begin{tabular}{|c|c|c|c|c|c|c|}
\hline $\begin{array}{c}\text { Peak } \\
\#\end{array}$ & $\begin{array}{c}\text { RetTime } \\
\text { [min] }\end{array}$ & Type & $\begin{array}{l}\text { Width } \\
\text { [min] }\end{array}$ & $\begin{array}{c}\text { Area } \\
{\left[\mathrm{mAU}{ }^{*} \mathrm{~S}\right]}\end{array}$ & $\begin{array}{l}\text { Height } \\
{[\mathrm{mAU}]}\end{array}$ & $\begin{array}{c}\text { Area } \\
\quad 8\end{array}$ \\
\hline--1 & $----\infty$ & & ------ & $--\cdots-------$ & ---------- & ------ \\
\hline 1 & 4.844 & $\mathrm{PP}$ & 0.0678 & 574.38422 & 129.29517 & 3.5669 \\
\hline 2 & 6.231 & $\mathrm{VB}$ & 0.1358 & $1.55289 \mathrm{e} 4$ & 1890.81689 & 96.4331 \\
\hline Total & : & & & $1.61032 \mathrm{e} 4$ & 2020.11206 & \\
\hline
\end{tabular}

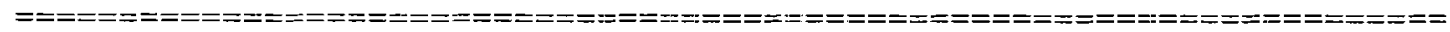
$\star * *$ End of Report 


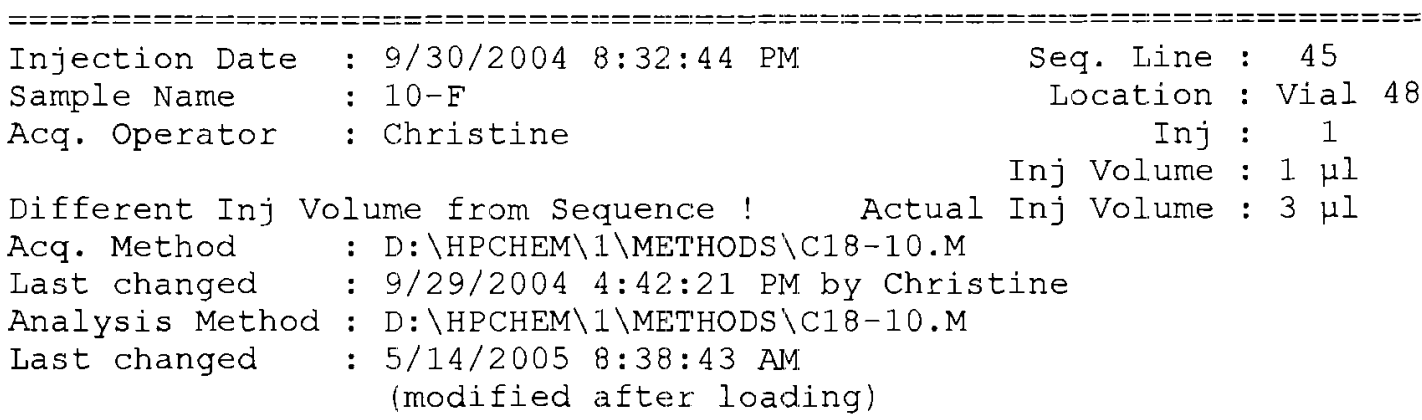

$\mathrm{C} 18 / \mathrm{MeCN}-\mathrm{H} 2 \mathrm{O}-\mathrm{TEA}$

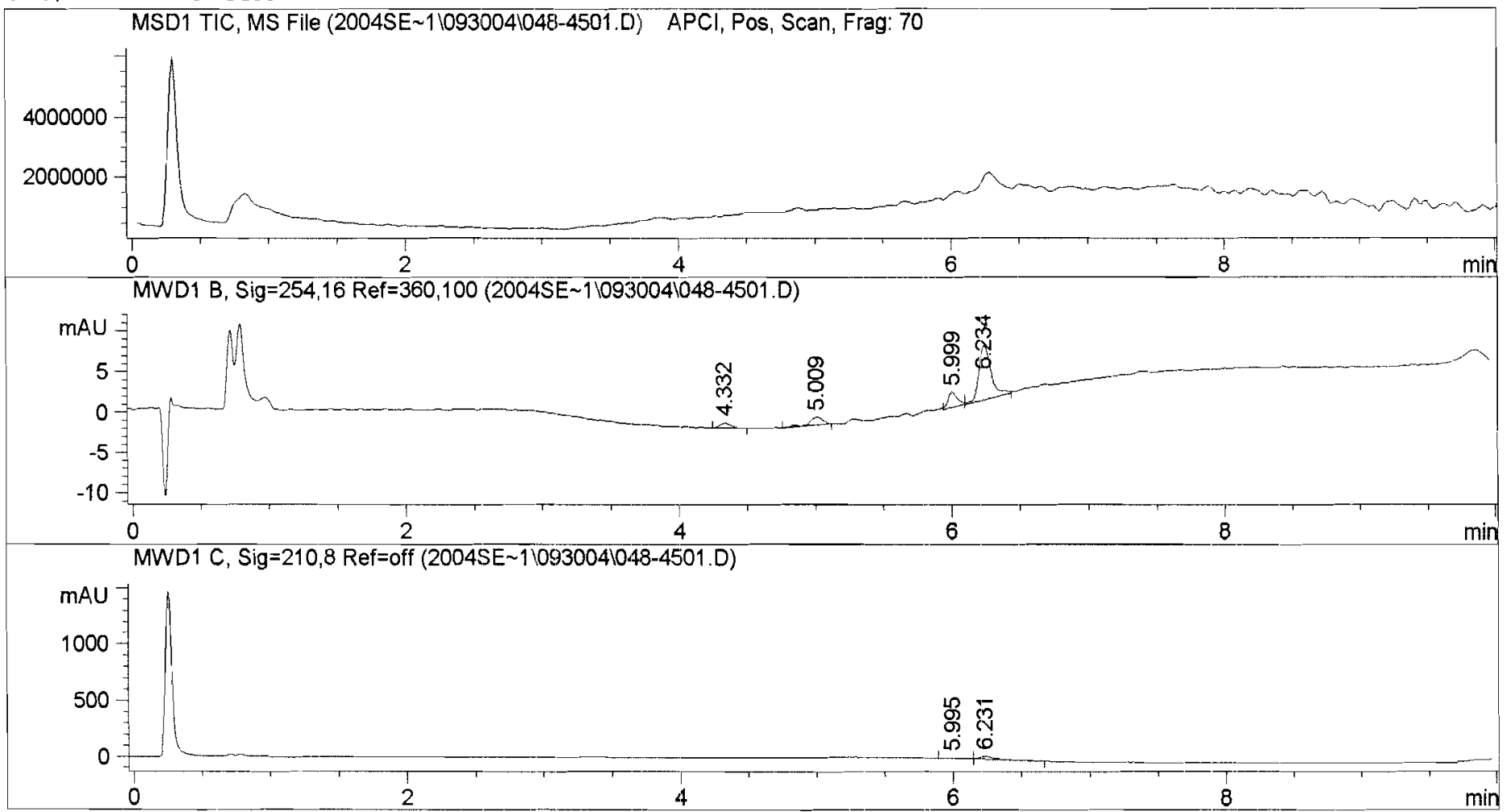

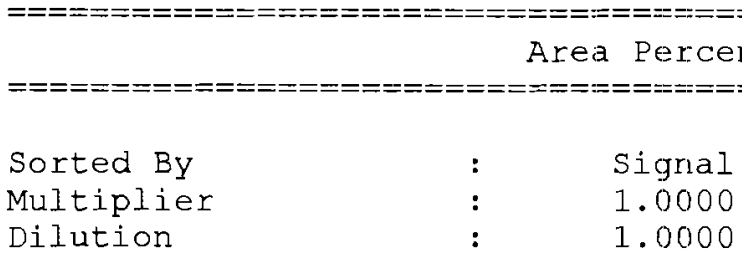

Signal 1: MSDI TIC, MS File

Signal 2: MWD1 B, Sig=254, 16 Ref $=360,100$

\begin{tabular}{|c|c|c|c|c|c|c|}
\hline $\begin{array}{c}\text { Peak } \\
\#\end{array}$ & $\begin{array}{c}\text { RetTime } \\
\text { [min] }\end{array}$ & Type & $\begin{array}{c}\text { Width } \\
\text { [min] }\end{array}$ & $\begin{array}{c}\text { Areal } \\
{\left[\mathrm{mAU}{ }^{\star} \mathrm{s}\right]}\end{array}$ & $\begin{array}{l}\text { Height } \\
\text { [mAU] }\end{array}$ & $\begin{array}{c}\text { Area } \\
\frac{8}{8}\end{array}$ \\
\hline & & & & & & \\
\hline 1 & 4.332 & PP & 0.0906 & 3.94595 & $6.52065 e-1$ & 6.2561 \\
\hline 2 & 5.009 & $\mathrm{PP}$ & 0.1098 & 7.30685 & 1.01786 & 11.5847 \\
\hline 3 & 5.999 & PV & 0.0651 & 8.70588 & 1.98770 & 13.8028 \\
\hline 4 & 6.234 & VB & 0.0962 & 43.11456 & 6.77967 & 68.3563 \\
\hline
\end{tabular}

Totals :

$63.07324 \quad 10.43729$ 


\begin{tabular}{|c|c|c|c|c|c|c|}
\hline $\begin{array}{c}\text { Peak } \\
\quad \#\end{array}$ & $\begin{array}{c}\text { RetTime } \\
\text { [min] }\end{array}$ & Type & $\begin{array}{l}\text { Width } \\
\text { [min] }\end{array}$ & $\begin{array}{c}\text { Area } \\
{\left[\mathrm{rnAU}^{\star} \mathrm{s}\right]}\end{array}$ & $\begin{array}{l}\text { Height } \\
\text { [mAU] }\end{array}$ & $\begin{array}{c}\text { Area } \\
\text { 是 }\end{array}$ \\
\hline---1 & & & & ---------ー & -------- & --------1 \\
\hline 1 & 5.995 & BV & 0.0971 & 73.52195 & 10.31844 & 25.4732 \\
\hline 2 & 6.231 & VB & 0.1055 & 215.10274 & 30.04976 & 74.5268 \\
\hline Total & Ls : & & & 288.62469 & 40.36820 & \\
\hline
\end{tabular}

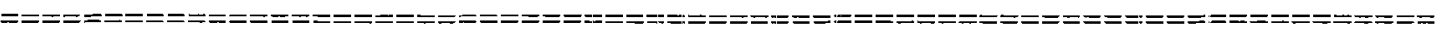

** End of Report ** 